
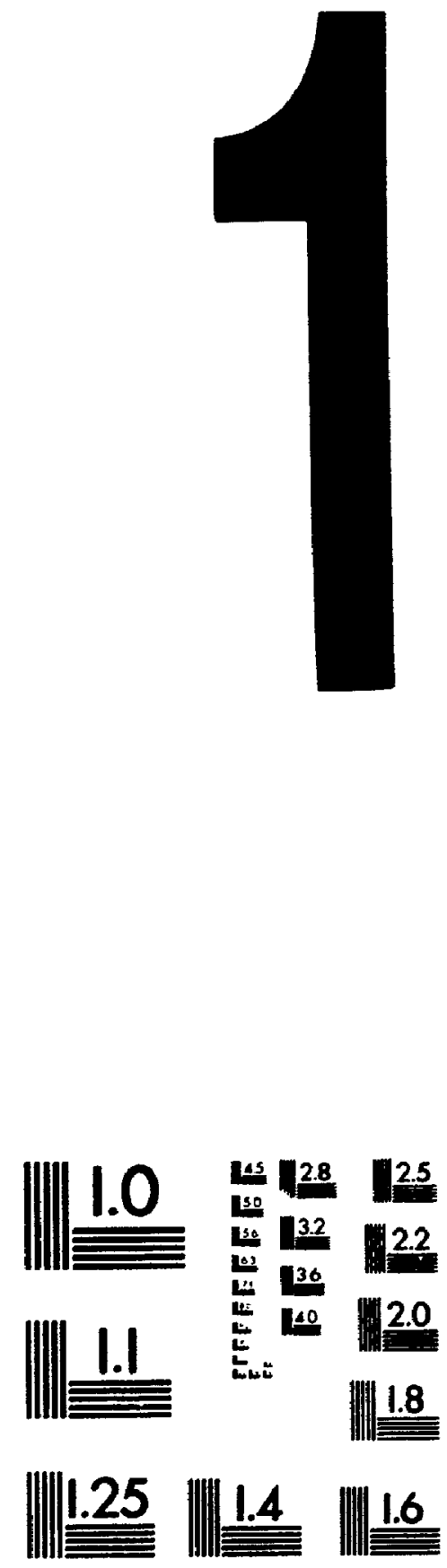

MICROCOPY RESOLUTION TEST CHART

NATIONAL BUREAU OF STANDARDS

STANDARD REFERENCE MATERIAL 1010a

(ANSI and ISO TEST CHART NO 2) 
National Librany

of Canada

Canadian Theses Service

Ottawa. Canada

KIA ON4
Bibliothèque nationale

du Canada

Service des thèses canadiennes
NOTICE

The quality of this microform is heavily dependent upon the quality of the original thesis submitted for microfilming. Every effort has been made to ensure the highest quality of reproduction possible.

If pages are missing, contact the university which granted the degree.

Some pages may have indistinct print especially if the original pages were typed with a poor typewriter ribbon or if the university sent us an interior pholocopy.

Reproduction in full or in pan of this microform is governed by the Canadian Copyright ACt, R.S.C. 1970, C. C-30, and subsequent amendments.

\begin{abstract}
AVIS
La qualité de cette microforme dépend grandement de la qualité de la thése soumise au microfilmage. Nous avons tout fait pour assurer une qualité supérieure de reproduction.

S'il manque des pages, veuillez communiquer avec l'université qui a conféré le grade.

La qualité d'impression de certaines pages peut laisser a désirer, surtout si les pages originales ont été daclylogra phiées à l'aide d'un ruban usé ou si l'universilé nous a lail parvenir une photocopie de qualité inlérieure.
\end{abstract}

La reproduction, méme partielle, he cette microforme est soumise a la Loi canadienne sur le droil d'auleur, SRC 1970 , c. C-30, et ses amendements subséquents. 


\title{
THE CHARACTERISTICS AND IMPORTANCE OF THE AIR TRAFFIC CONTROLLER'S MENTAL MODEL
}

\author{
by \\ RICHARD H. MOGFORD, M.A. \\ A thesis submitted to \\ the Faculty of Graduate Studies and Research \\ in partial fulfilment of \\ the requirements for the degree of \\ Doctor of Philosophy \\ Department of Psychology
}

Carleton University

Ottawa, Ontario

October 12, 1990

(c) copyright

1990, Richard H. Mogford 
Onawa. Canada

KIA ONA

\section{NOTICE}

The quality of this minfoform is heavily dependent upon the quality of the original thesis submitted for microffilming. Every effort has been made to ensure the highest quality of reproduction possible.

If pages are missing. contact the university which granted the degree.

Some pages may have indistinct print especially if the original pages were typed with a poor typewriter ribbon or if the university sent us an inferior photocopy.

Reproduction in full or in part of this microform is governed by the Canadian Copyright ACt. R.S.C. 1970. C.C-30, and subsequent amendments.

\begin{abstract}
AVIS
La qualité de cette microforme dépend grandement de la qualite de la these soumise au microfimage. Nous avons tout fait pour assurer une qualité supérieure de reproduction.

Sil manque des pages. veuillez communiquer avec runiversité qui a contéré le grade.

La qualité dimpression de certaines pages peut laisser à désirar. surtout si les pages originales ont été dactylogra. phiées à laide d’un nuban usé ou si luniversité nous a fait parvenir une photocopie de qualite intérieure.

La reproduction, méme partielle, de cette microlorme est soumise a la Loi canadienne sur le droit dauteur. SRC 1970. c. C-30. et ses amendements subséquents.
\end{abstract}

I SBN $\quad 0-315-60445-X$ 
The undersigned hereby recommend to the Faculty of Graduate Studies and Research acceptance of the thesis

THE CHARACTERISTICS AND IMPORTANCE OF THE AIR TRAFFIC CONTROLLER'S MENTAL MODEL

\author{
submitted by \\ RICHARD H. MOGFORD, M.A.
}

in partial fulfilment of the requirements

for the degree of Doctor of Philosophy

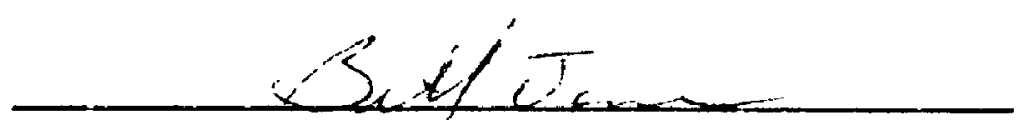

Chair, Department of Psychology

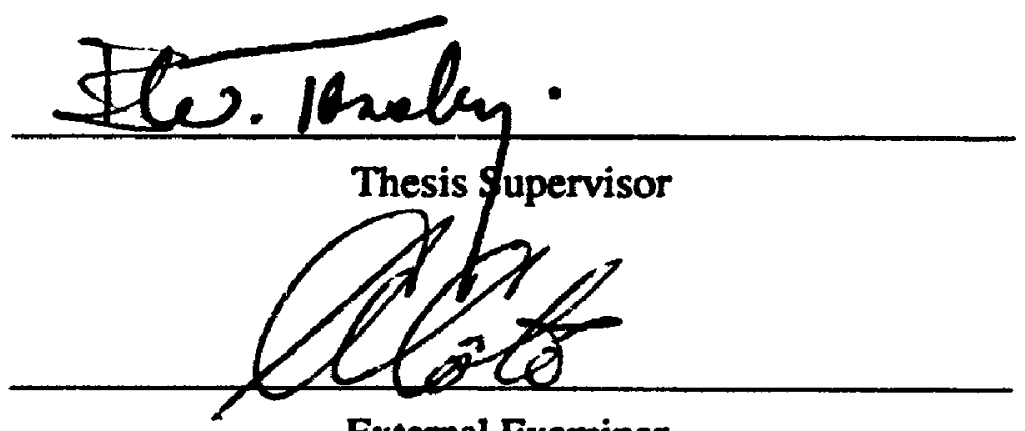

External Examiner

Carleton University 


\begin{abstract}
A research study was conducted into the nature and importance of the air traffic controller's mental model (or "picture"). A mental model is an internal representation of knowledge which helps describe, explain, and predict system behaviour.

A preliminary study employed a microcomputer-based radar simulation. Subjects included five experienced controllers, three university students, and four simulator "pilots". Controllers had significantly better accuracy and consistency of recall of screen information. They also prioritized data in the same way as in other studies, showed evidence of a more resilient picture, were more accurate with one of the prediction tasks, and showed a dichotomizing decision-making style. There were no significant differences between subjects with regard to other tasks addressing cognitive organization and spatial skills. However, all subjects showed a task-related priority of recall of screen information and there was some evidence of three-dimensional spatial visualization.
\end{abstract}

Two additional experiments were completed with controller trainees to determine if the accuracy of the picture (as measured by a recall task) is an important skill for air traffic control. Experiment 1 focused on radar control. Subjects ( $N=37$ ) were given a picture assessment test, an aptitude test battery, and memory tests. A number of the variables, including those relating to picture accuracy, emerged as predictors of both academic and simulation final grades. A discriminant function successfully categorized subjects who passed or failed. Experiment 2 focused on a procedural class $(\mathrm{N}=17)$. It was again possible to predict academic and simulation performance although the picture assessment variables played a lesser role.

Based on this research, a number of observations are made about the characteristics and importance of the controller's mental model. The main finding is that the accuracy of the picture is important for radar air traffic control. It is also concluded that recall measures are useful tools for exploring the mental model and that cognitive style may be 
an important factor for success in training. It is recommended that further research be conducted to explore the controller's mental model and that the experimental findings be applied to the development of selection tests. 


\section{ACKNOWLEDGEMENTS}

The author wishes to acknowledge the invaluable support and guidance supplied by his thesis advisor

Brian Tansley, Ph.D.

and the assistance provided by the following individuals and organizations which made this research possible:

Mr. Robert Armstrong Manager

Air Traffic Services Research and Experimentation Centre Transport Canada Hull, Quebec

Mr. Walter Bulbeck Director Air Traffic Services Training Transport Canada Training Institute Cornwall, Ontario

Mr. Dick Campbell

Air Traffic Services Transport Canada Ottawa, Ontario

William Petrusic, Ph.D. Psychology Department Carleton University

Tom Tombaugh, Ph.D. Psychology Department Carleton University 


\section{TABLE OF CONTENTS}

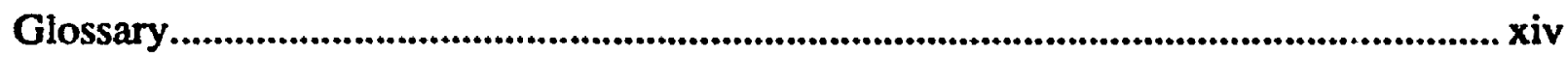

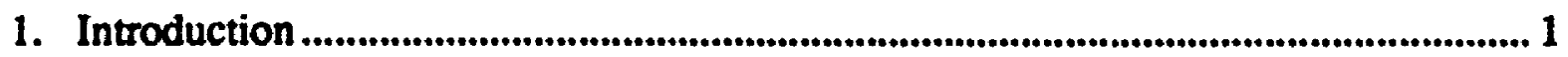

1.1 Definitions of the Mental Model ................................................................... 4

1.2 The Characteristics of the Mental Model ..................................................... 5

1.3 Theory and Method in Research on Mental Models ..................................... 8

1.4 Review of Studies on the Controller's Mental Model...................................... 12

1.5 Evaluation of Previous Research.................................................................. 24

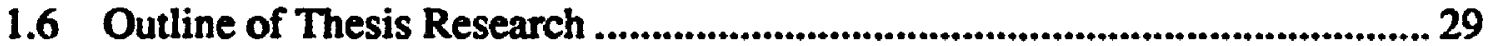

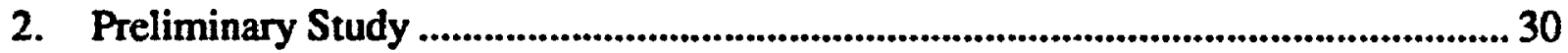

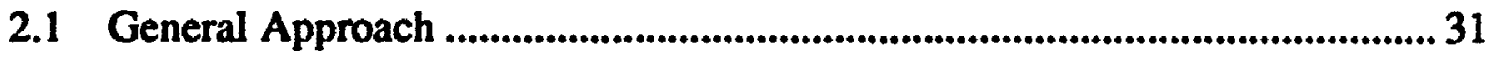

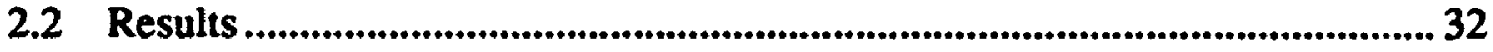

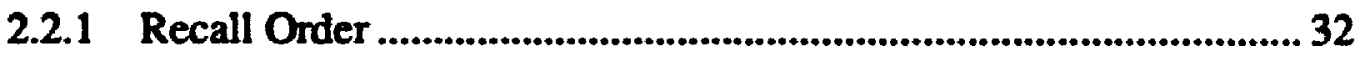

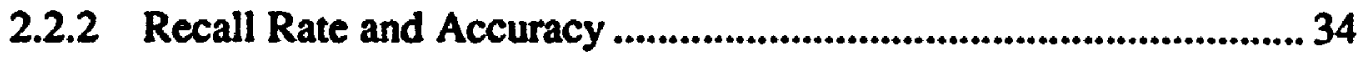

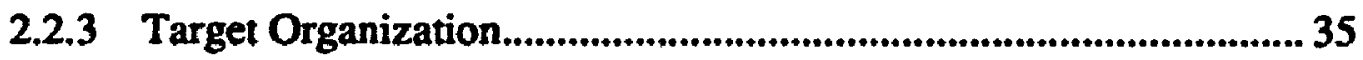

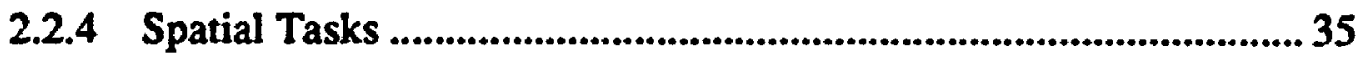

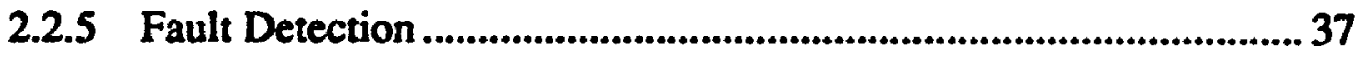

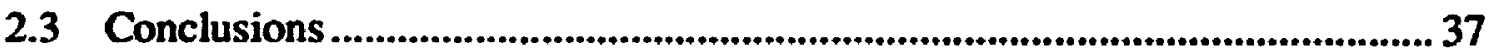

2.4 Rationale for Further Experiments.......................................................... 38

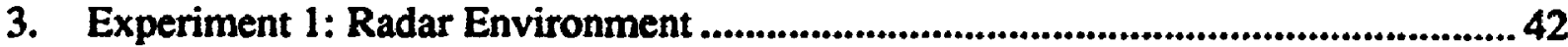

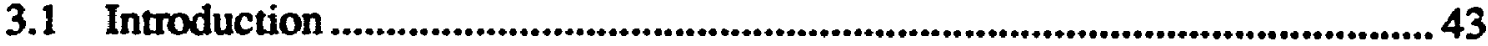

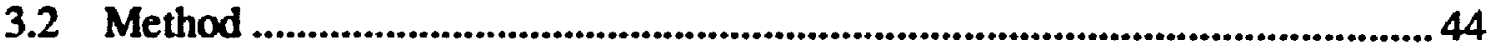

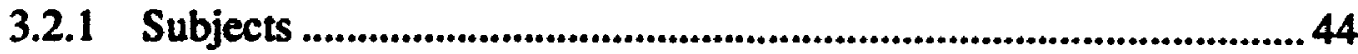

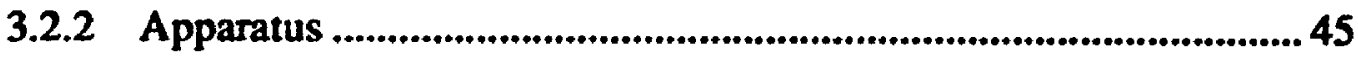

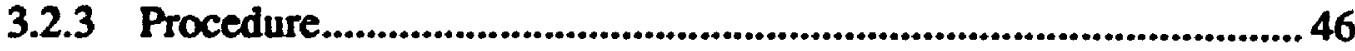

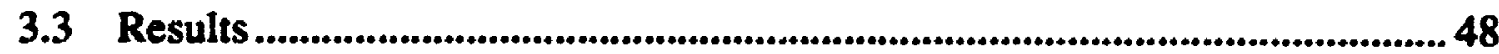

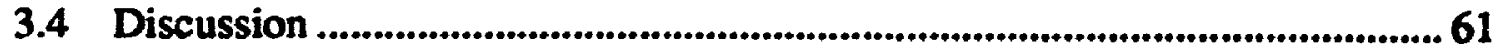

4. Experiment 2: Procedural Environment........................................................ 70

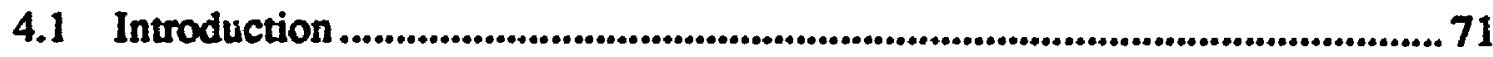

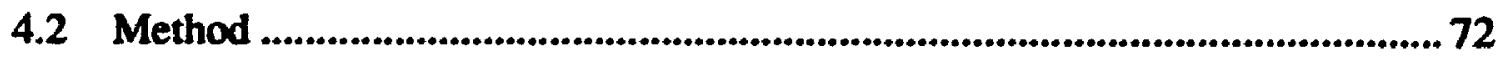

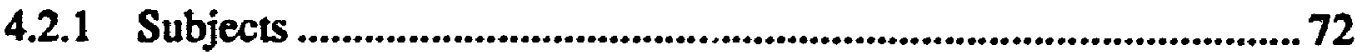

4.2.2 Apparatus ........................................................................................... 72

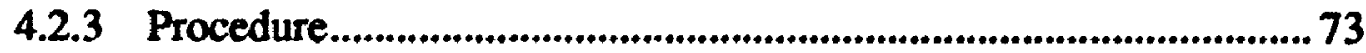

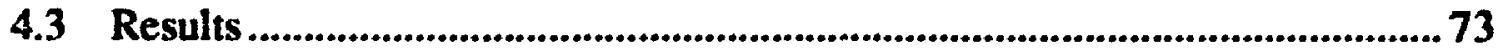




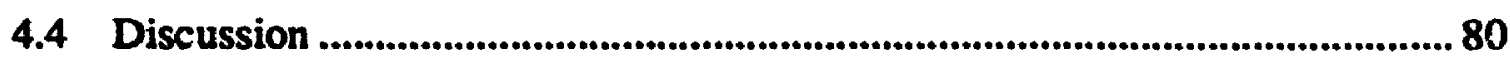

5. General Discussion and Conclusions...........................................................86

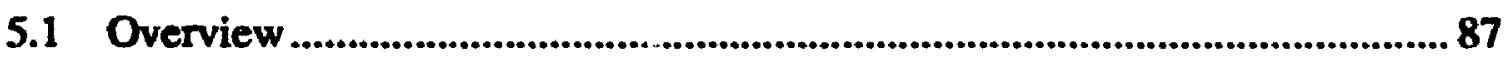

5.2 Theoretical Issues................................................................................ 87

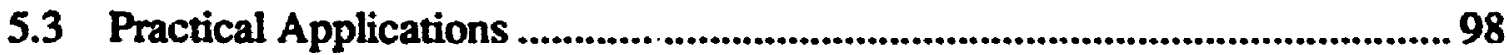

5.4 Suggestions for Further Research ............................................................... 100

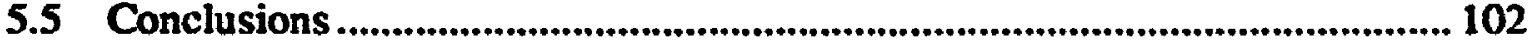

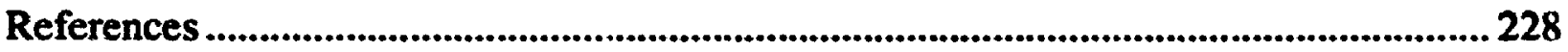




\section{LIST OF TABLES}

Table 1. Information required by the sector controller ................................................. 7

Table 2. Per cent of aircraft attributes correctly recalled ................................................ 18

Table 3. Per cent of aircraft attributes correctly recalled from the blank flight strip task

Table 4. Per cent of aircraft not recalled in the map condition

Table 5. Per cent of aircraft not recalled in the blank page condition

Table 6. Number of aircraft attributes correctly recalled.

Table 7. The experimental conditions included in the Preliminary Study.

Table 8. Variables used in the analysis of Experiment 1 and 2 data.

Table 9. Variables for which a significant difference existed between the first and second radar classes

Table 10. Pearson product-moment correlations between memory and picture assessment measures.

Table 11. Correlations of criterion measures.

Table 12. Regression of average academic test score over selected predictor variables.

Table 13. Average simulation performance (three trials) regressed over selected predictor variables.

Table 14. Average simulation performance (three trials) regressed over picture assessment variables.

Table 15. Stepwise discriminant function analysis for the prediction of pass/fail outcome in the radar training class.

Table 16. Pearson product-moment correlations between memory and picture assessment measures.

Table 17. Correlations of criterion measures.

Table 18. Average academic performance regressed over selected predictor variables.

Table 19. Average simulation performance (three trials) regressed over selected predictor variables.

Table A-1. The experimental conditions included in the Preliminary Study. 
Table A-2. Kendall rank-order correlations between of onder of recall and ratings of importance............................................................................................. 126

Table A-3. Criteria used for establishing ballpark accuracy. ......................................... 128

Table A-4. Aircraft attributes correctly recalled (as per cent correct of total available) in order of priority

Table A-5. Support or rejection of hypotheses stated for the Preliminary Study. 146 


\section{LIST OF FIGURES}

Figure 1. Norman's concept of a human-machine system.............................................. 3

Figure 2. The components and cognitive abilities necessary for the maintenance and use of a mental model................................................................................7

Figure 3. Distinctions among domains of mental models. .............................................10

Figure 4. A sample stimulus from the Landis, et al. (1967) study. ................................ 16

Figure 5. The data block as displayed on an air traffic control radar screen................. 26

Figure 6. Mean per cent correct for aircraft attributes recalled during picture assessment. ............................................................................................................56

Figure 7. Mean per cent correct for aircraft attributes recalled during picture

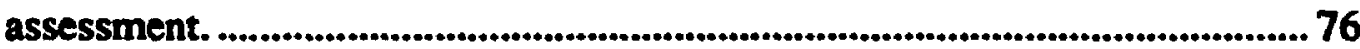

Figure 8. The relationships between the air traffic controller's mental structures........ 104

Figure A-1. The trigonometry of Pinker's (1980) mental imagery experiment (as applied to air traffic control)..................................................................... 110

Figure A-2. Frequency of recall (in per cent) of aircraft in first five targets recalled.

Figure A-3. The rate of recall (per cent of total aircraft attributes correctly recalled per minute).

Figure A-4. Absolute accuracy of recall of aircraft two- and three-dimensional location

Figure A-5. Ballpark accuracy in recalling aircraft 2D location and 3D location (within $5 \mathrm{~nm}$ ) using RMS error calculation.

Figure A-6. Pearson product-moment correlations of subject judgements of relative interobject distance with actual two- and three-dimensional interobject distances

Figure A-7. The differences in RMS error for plotting the 2 minute positions of arriving versus intersecting aircraft.

Figure A-8. The number of pilot errors detected of any type and the average response time to detect them. 


\section{LIST OF APPENDICES}

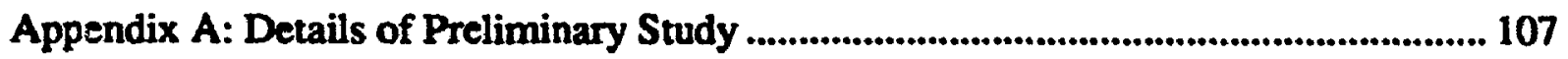

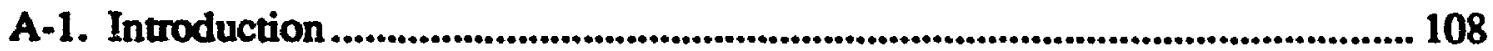

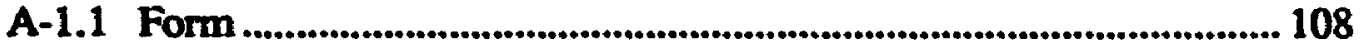

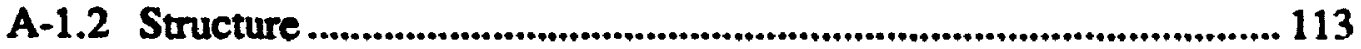

A-1.3 Parameters .................................................................................................... 113

A-1.4 Predicting Aircraft Location.............................................................. 114

A-1.5 Fault Detection ................................................................................ 114

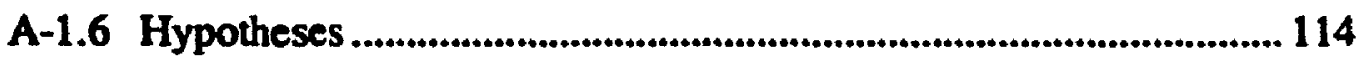

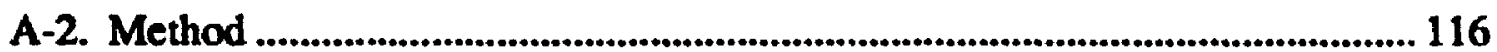

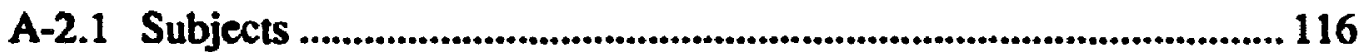

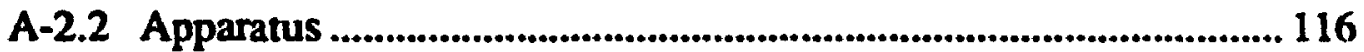

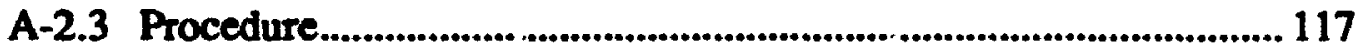

A-2.3.1 Practice Exercise ............................................................118

A-2.3.2 Recall Exercise................................................................ 118

A-2.3.3 Subjective Altitude Judgements....................................... 120

A-2.3.4 Practice Plotting Exercise................................................ 120

A-2.3.5 Modeling and Prediction of Overflying Aircraft and Arrivals ....................................................................120

A-2.3.6 Modeling and Prediction of Intersecting Aircraft ............ 121

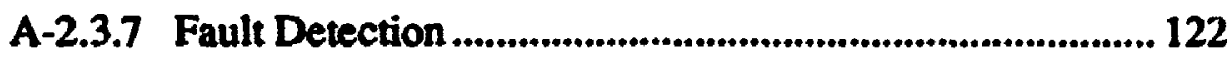

A-3. Results and Discussion..................................................................................... 122

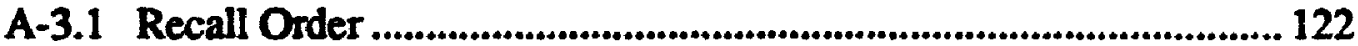

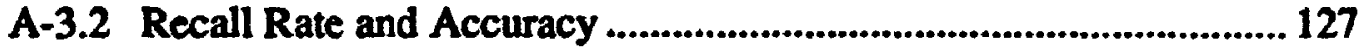

A-3.3 Target Organization........................................................................ 132

A-3.4 Interobject Distance Judgements................................................... 133

A-3.5 Subjective Altitude Judgements................................................... 134

A-3.6 Head Tums ........................................................................................ 137

A-3.7 Response Time ................................................................................ 137

A-3.8 Model Building: All Trials..................................................................... 138

A-3.9 Prediction: All Trials............................................................................ 139

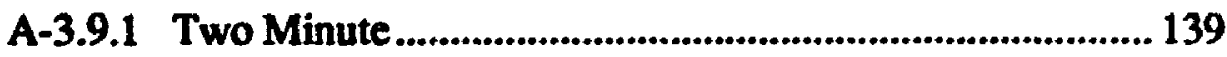

A-3.9.2 Five Minute ............................................................ 140 
A-3.9.3 Present Position and 2 Minute Prediction:

Arrivals versus Overflights

A-3.9.4 Present Position and 2 Minute Prediction:

Conflicts versus Non-Conflicts ........................................ 143

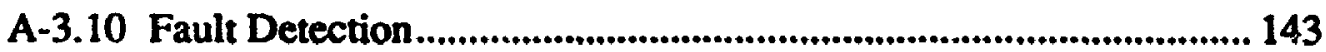

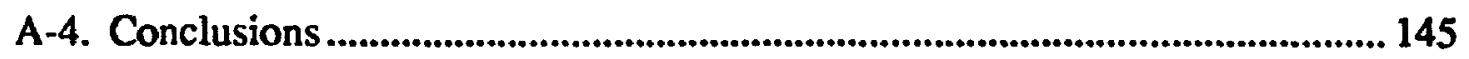

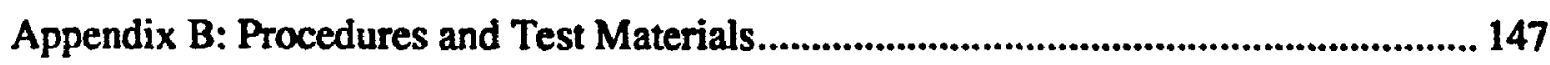

Appendix B-1: Experiment 1 .............................................................................. 147

Appendix B-1.1: Student Procedure............................................................... 147

Appendix B-1.2: Instructor Procedure ............................................... 150

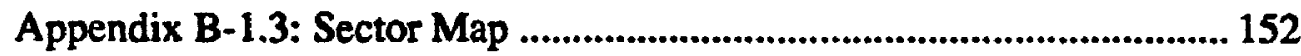

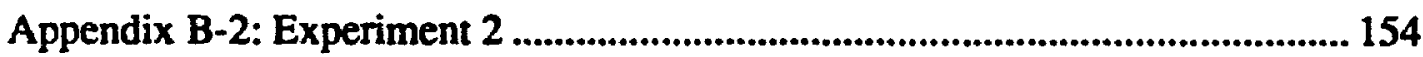

Appendix B-2,1: Student Procedure.............................................................. 154

Appendix B-2.2: Instructor Procedure ................................................ 156

Appendix B-2.3: Sector Map ....................................................................... 158

Appendix B-3: Experiments 1 \& 2 Tests and Criterion Measures....................... 160

Appendix B-3,1: Differential Aptitude Test ........................................... 160

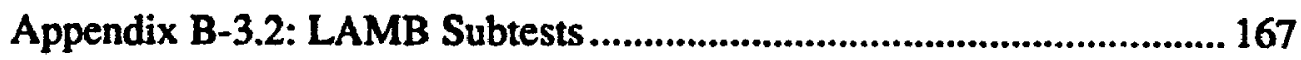

Appendix B-3.3: IFR Training Report ............................................. 176

Appendix B-3.4: ATC Student Rating Form .......................................178

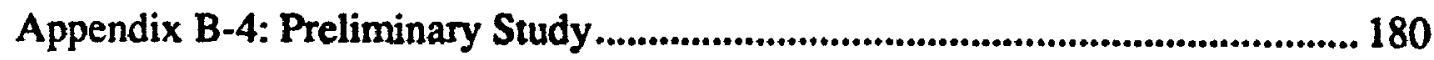

Appendix B-4.1: Experimental Protocol ................................................ 180

Appendix B-4.2: Sector Map ................................................................... 190

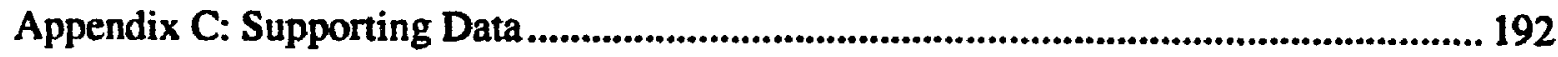

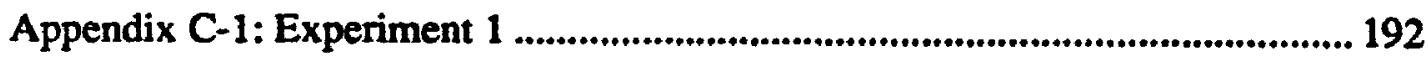

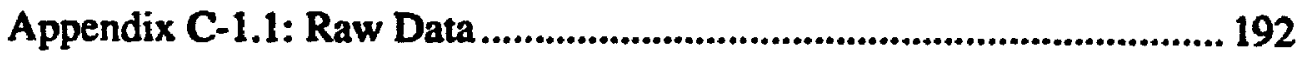

Appendix C-1.2: Univariate Outliers .................................................. 196

Appendix C-1.3: Skewness \& Kurtosis of Variables.............................. 200

Appendix C-1.4: Correlation Matrix ....................................................... 202

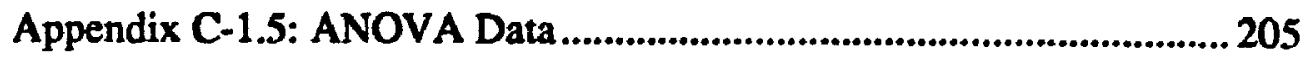

Appendix C-2: Experiment 2 ................................................................................. 207

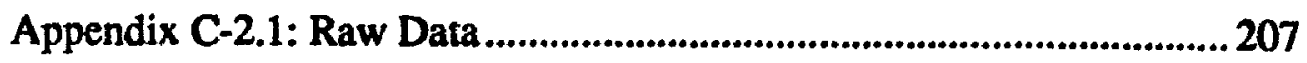

Appendix C-2.2: Univariate Outliers ........................................................210

Appendix C-2.3: Skewness \& Kurtosis of Variables ............................. 213 
Appendix C-2.4: Correlation Matrix........................................................ 215

Appendix C-2.5: ANOVA Data ................................................................ 218

Appendix C-3: Experiments 1 \& 2: Conclusions: t-Test Data.............................. 220

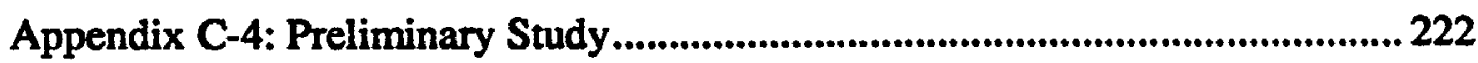

Appendix C-4.1: Sector Map .................................................................... 222

Appendix C-4.2: $\mathrm{t}$-Test Data ........................................................................ 224

Appendix C-4.3: ANOVA Data ................................................................... 226 


\section{GLOSSARY}

2D ....................... two-dimensional

3D ........................ three-dimensional

ANOVA .............. analysis of variance

ATC..................... air traffic control

ACAD.................. average of 6 academic tests in ATC training program

AGE.................... age of subject

ALT ..................... picture assessment: per cent of altitudes correctly recalled

AR ..................... DAT Abstract Reasoning

AVG OALL......... average of single overall ratings on global rating form

AVG PICT........... average of pizture ratings on global rating form

AVG RATE......... average of 29 specific factors on global rating form

b. regression coefficient (beta weight)

B.......................... standardized regression coefficient

$\mathrm{CF} 1+\mathrm{CF} 2$.............. sum of first two trials for Complex Figure recall

$\mathrm{CF} 4-\mathrm{CF} 1$............... difference of last trial and first trial for Complex Figure recall

CFT1 + CFT2 2........ sum of response times for first two trials for Complex Figure recall

CFT4-CFT1 difference of last and first trial response times for Complex Figure recall

CS\&A ................. DAT Clerical Speed and Accuracy

DAT.................... Differential Aptitude Test

df ........................ degrees of freedom

EDUC.................. total years of education

FR1+FR2 ............. sum of first two trials for List Learning free recall

FR5-FR1 ............... difference between last and first trial for List Learning free recall

HDG ................... picture assessment: per cent of headings correctly recalled (ballpark)

IFR........................ instrument flight rules

IDENT ................. picture assessment: per cent of identifiers correctly recalled

LAMB ................ Learning and Memory Battery

$M$....................... mean (average)

MAX.................... maximum

MIN .................... minimum

MCAT ................. Multiplex Controller Aptitude Test

MDS ................... multidimensional scaling

MR ..................... DAT Mechanical Reasoning 
MS

mean square

NA DAT Numerical Ability

NASA National Aeronautic and Space Administration

nm nautical mile

NOAC. picture assessment: per cent of aircraft recalled

p. probability

POS picture assessment: per cent of locations correctly recalled (ballpark)

r........ Pearson product-moment correlation coefficient

R. \& E. Centre. Transport Canada Research and Experimentation Centre

RATE picture assessment: number of aircraft attributes recalled per minute

RMS root mean square

SE standard error

SIM average of 3 simulator final exam scores (4 in procedural phase)

SP picture assessment: per cent of speeds correctly recalled (ballpark)

SR DAT spatial reasoning

SS sum of squares

t. Student's t

T Kendall rank-order correlation coefficient

TCTI Transport Canada Training Institute

TRACON Terminal Radar Area Control

VR DAT Verbal Reasoning

VR+NA sum of VR and NA

WKMTOT sum of errors of two and three back trials for Working Memory 


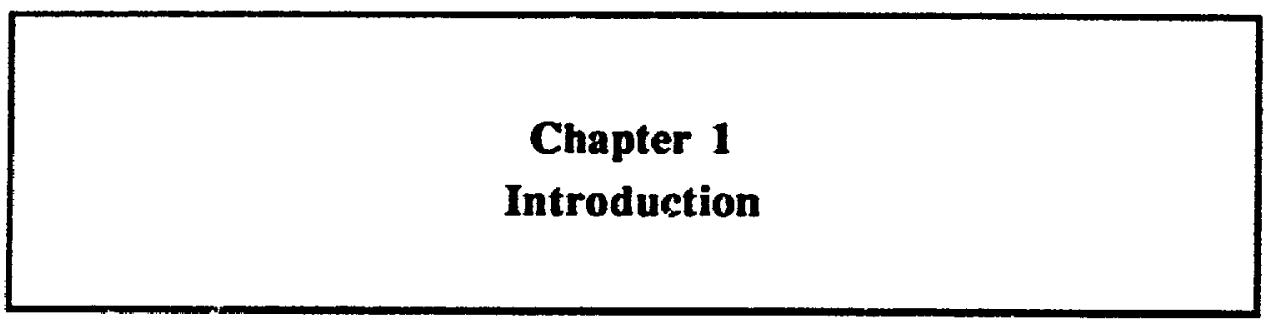


Although the world appears to be solidly "out there", it is actually a mental construction. Physical stimuli give rise to sensory information which, when combined with knowledge, results in the consciousness of an organized and largely predictable environment. Each of us carries with us a mental model of reality which enables us to understand the "booming, buzzing confusion".

Our technological evolution has allowed increasing amounts of control over the appearance of our physical surroundings. With the advent of computers and visual display terminals we are now able to create artificial information environments for various purposes. Interest is growing over how best to portray these "worlds" for the most effective support of complex tasks.

Safe air traffic control (ATC) depends on the operator's grasp of a complicated, changing situation. It is important that the images presented on radar displays be compatible with the controller's mental model of aircraft in airspace. The controller should not have to translate system information to fit the model.

Wickens (1984) observed that there are three levels of representation in such a system: (a) the physical system, (b) the operator's internal representation of the system, and (c) the interface between (a) and (b), that is, "the display surface upon which changes in the system are presented to 'update' the operator's internal model and to form the basis for control action and decision" (Wickens, 1984, p. 179). He stressed that training is the method for creating the operator's internal or mental model of a system.

A more detailed concept of the human-machine relationship is that of Norman (1986). He distinguished between the "Design Model", the "System Image", and the "User's Model" (see Figure 1). The Design Model is the planner's conceptual model of the intended system, taking into account the user's background and abilities. The domain, processes, and equipment to be controlled are also undoubtedly included in the designer's considerations. 


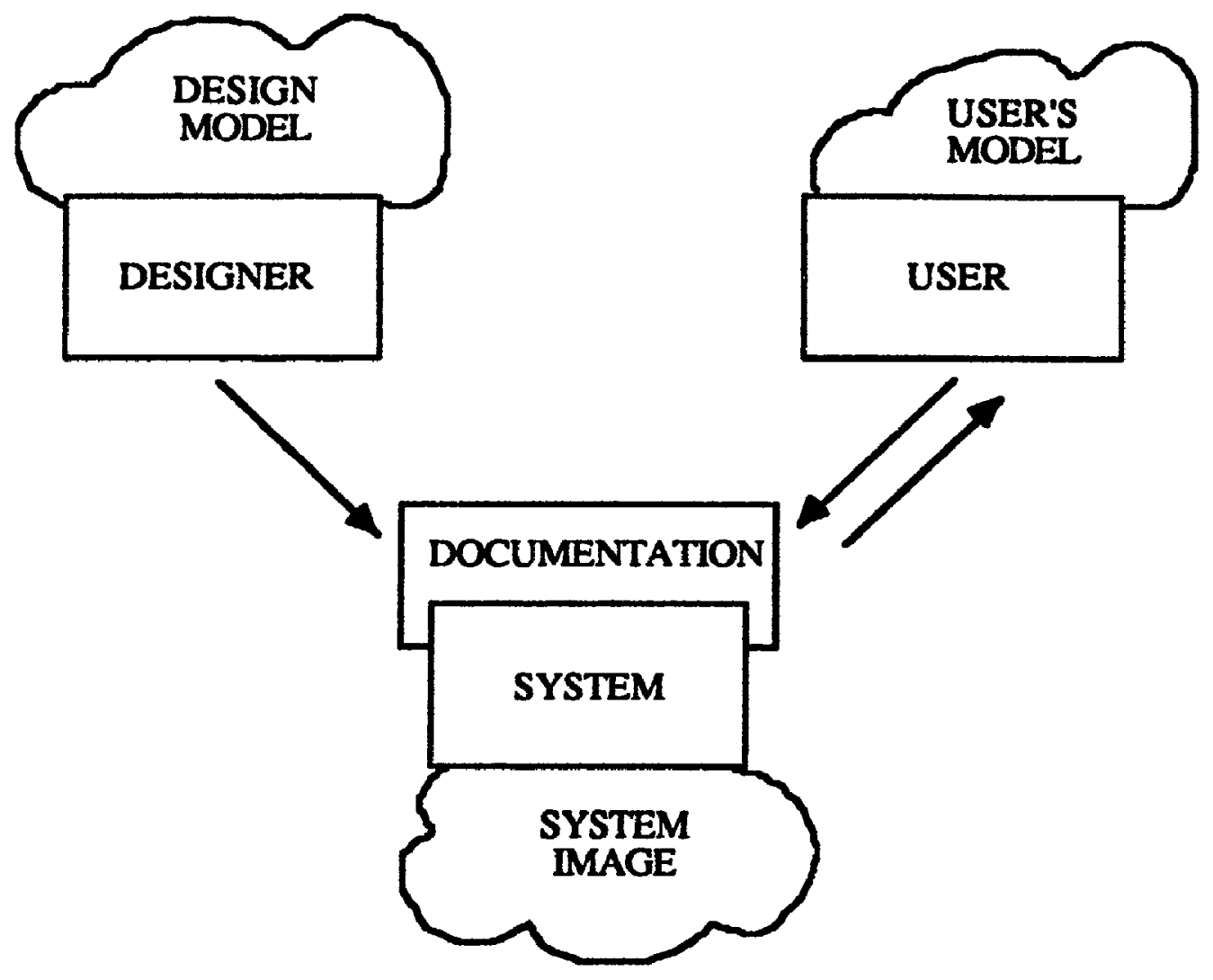

Eiqure 1. Norman's concept of a human-machine system. Note. From User Centered System Design (p. 46) by D. A. Norman and S. W. Draper (Eds.), 1986, Hillsdale, NJ: Lawrean'e Erlbaum. 
The operator's mental model of the system is called the User's Model. However, it is not derived directly from the Design Model but from the System Image, the physical expression of the hardware and software decided upon by the designer. Norman (1986) stated that the goal is for the User's Model (gained through interaction with the system) to he compatible with the Design Model. He suggested that the System Image must be explicit, intelligible, and consistent.

Knowing the training program and the characteristics of the System Image can lead to assumptions about the user's mental model. However, the operator's disposition and interests have an influence on the model and it may not be turn out to be the same as what the instructor or designer had in mind. Only by directly investigating the air traffic controller's conceptions can an accurate understanding of the mental model emerge.

\subsection{Definitions of the Mental Model}

A mental model is a hypothetical construct which refers to the operator's ideas about a system and what it is controlling. Wickens (1984) noted that the internal model helps guide and explain the operator's information gathering strategies, is employed to design the necessary control inputs to achieve future goals, and is the basis of the operator's expectancies about covarying events and so is useful in the detection of problems. Another statement of this idea is:

Mental models are the mechanisms whereby humans are able to generate descriptions of system purpose and' form, explanations of system functioning and observed system states, and predictions of future system states. (Rouse \& Morris, 1986, p. 351)

Norman (1986) described this concept in the following:

Mental models seem a pervasive property of humans. I believe that people form internal, mental models of themselves and of the things and people with whom they interact. These models provide predictive and explanatory power for understanding the interaction. Mental models evolve naturally through interaction with the world and with the particular system under consideration. These models are highly affected by the nature of the interaction, coupled with the person's prior knowledge and understanding. The models are neither 
complete nor accurate, but nonetheless they function to guide much human behavior. (p. 46)

In a similar vein, Wilson and Rutherford (1990) related the quality of the mental model to operator efficiency:

a mental model is a representation formed by a user of a system and/or task, based on previous experience as well as current observation, which provides most (if not all) of their subsequent system understanding and consequently diciates the level of task performance. (p. 619)

In the case of air traffic control the radar screen and other displays provide aircraft information which the controller is somehow able to use to construct a mental model of airspace, the objects within it, and the rules which govern their dynamics. The fact that some kind of internal model exists is demonstrated by the controller's ability to project the future positions and speeds of aircraft in order to anticipate possible conflicts. A controller is able to continue to do this for a period of time even if deprived of the radar display (Whitfield, 1979).

\subsection{The Characteristics of the Mental Model}

It is tempting to assume that the controller's model is simply a visualization of aircraft, navigational markers, terrain, and other pictorial elements of the system under supervision. However, recalling that the purpose of the mental model is not only to describe the ATC situation but also to support the "safe, orderly, and expiditious flow of air traffic" (Hopkin, 1980, p. 548), it becomes evident that there must be other aspects of the modil which are not visually represented such as rules, procedures, and aircraft performance data.

Rasmussen (1979) described mental models as having different levels of abstraction including physical form, physical function, functional structure, abstract function, and functional meaning. Johnson-Laird (1983) distinguished berween physical and conceptual mental models. There may be a number of mental models of a system ranging from the "concrete" (an analogue, visual image) through various forms depicting functional 
relationships and operating rules, to a level subsuming the overall meaning and purpose of the system (Wilson \& Rutherford, 1990).

On the left side of Figure 2 are illustrated some of the components which may comprise a user's mental model of an air traffic control system. Each component may relate to one or more overlapping or distinct mental models. The right side of the diagram shows some of the cognitive resources which are required to support the use of the components. Although some type of model based on a visual image is probably involved in many air traffic control tasks, it may not be sufficient in itself for all aspects of the work. Other ikinds of verbal and numerical information must also be present and be put to use as needed.

To provis a more specific idea of the components of the air traffic controllers' mental model, a list of the information needed by the air traffic controller is shown in Table 1 (Whitfield \& Stammers, 1981). Although the table lists memory as the source of a few of these items, a little additional reflection suggests that long or short term memory would be involved to some degree in nearly every category.

The description of the mental model is not fully agreed upon. Wilson and Rutherford (1990) commented that "We have shown the several different interpretations of the concept and its utility to be a weakness, which militates against the widespread use of mental models in systems design" (p. 629). When this concept is applied to the study of a particular domain, the kind of findings which emerge will depend upon which aspect of the internal model is being emphasized.

This research will be limited to the consideration of some of the more observable aspects of the air traffic controller's mental model. However, it is hoped that enough evidence will be gathered to support a useful description and to suggest directions for further study. 


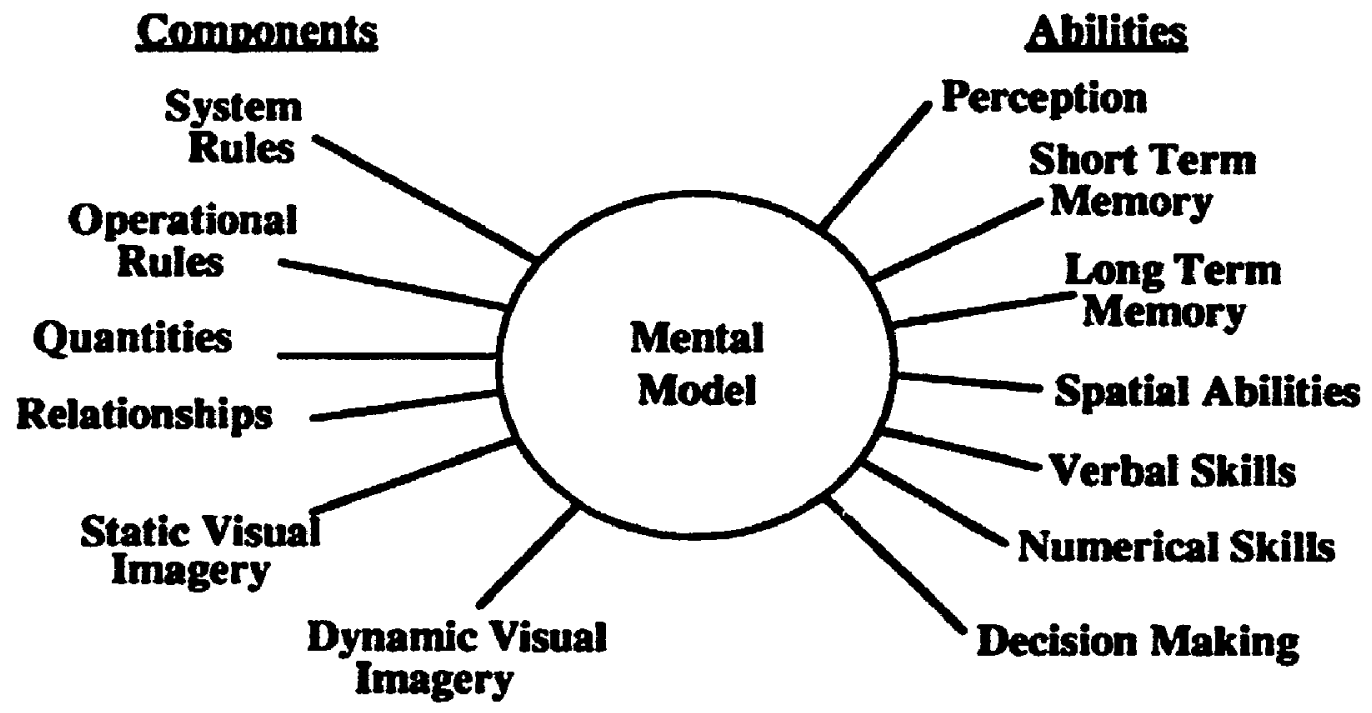

Eigure 2. The components and cognitive abilities necessary for the maintenance and use of a mental model.

\begin{tabular}{|l|l|}
\hline \multicolumn{1}{|c|}{ INFORMATION } & \multicolumn{1}{|c|}{ SOURCE(S) } \\
\hline Aircraft present position & Radar Display or via R/T \\
Aircraft present height & Radar display (SSR) or via R/T \\
Aircraft intention & Flight Plan display or via R/T \\
Separation standards & Memory or documentation \\
Route structure & Memory or map display \\
SSR code allocation & Display list \\
ATC operating instructions & Memory or documentation \\
Metcorological information & Display \\
Aeronautical information, permanent or temporary & \\
(e.g. range, frequency, and call sign of navigational & \\
aids; hazards to aircraft) & Display boards \\
Aircraft performance & Experience and documentation \\
Hand-over procedures & Standard or telephone \\
Adjacent Sector or ATC Unit R/T frequencies & Telephone or R/T \\
Emergency procedures & Memory or display lists \\
& Display lists \\
\hline
\end{tabular}

Table 1. Information required by the sector controller (UK) and possible sources of this information. Note. From The Analysis of Practical Skills_(p. 217) by W. T. Singleton (Ed.), 1981, Lancaster, UK: MTP. 


\subsection{Theory and Method in Research on Mental Models}

Rouse and Morris (1986) discussed some of the issues regarding research on mental models. They emphasized that a clear definition of terms is important in order to distinguish a mental model from knowledge about things in general. Accordingly, the themes of describing, explaining, and predicting the behaviour of a system or device were stressed.

Mental representations may be explored using verbal reports. They also may be approximated using inferential methods in two ways. "Empirical modeling" studies are used to mathematically describe the human operator by explaining the relationship of inputs to outputs. In constrained manual control systems, such as in the steering of large ships, it is possible to derive equations characterizing the operator's behaviour which provide inferences about the underlying mental model (Jagacinski \& Miller, 1978). Rouse \& Morris (1986) noted that it is important in empirical studies not to confuse the effects of perceptual and response processes with the operation of the mental model under study.

"Analytical modeling" is not based upon experimentation, but employs relevant theory and information about the human-machine system to construct a hypothetical model of the internal representation. As Norman (1983) observed, this becomes the observer's or researcher's model of the user's model. An analytical model may be created to explain the results obtained from empirical studies. However, even with a good rationale, it is still not possible to assume that the resulting analytical concept is the mental model; there may be more than one way of explaining the relationship of inputs to outputs.

In less constrained situations where it is not possible to create an equation describing mental operations, it is even more difficult to determine which analytical model best explains operator behaviour. The added fact that the user's underlying representations may actually be "messy, sloppy, incomplete and indistinct" (Norman, 1983, p. 14) suggests that our theorizing about them may never be precise and definitive. 
The fact that empirical or analytical modeling is sometimes required implies that humans do not have direct access to their mental models. Otherwise, why could we not merely ask people to describe their concepts? The reliability of verbal reports of cognitive activity has been debated for some time (Nisbett \& Wilson, 1971; Smith \& Miller, 1978; Ericksson \& Simon, 1980). Rouse and Morris (1986) commented that subjects may be aware of what they are thinking about but not about how they arrived at these thoughts. In addition, those who are experts in a certain domain may have learned their tasks so thoroughly that the procedures involved have become "automated" and are no longer verbally mediated during execution (Hammond, 1987; Smith \& Miller, 1978).

Moray (1980) described research which demonstrated that unconscious responses to the patterns and demands of the environment (learning how to operate a complex system, for example) result in more efficient behaviour.

It appears that learning, by which information becomes directly able to control behaviour without conscious decision making, is an effective way of bypassing the limitations of memory and consciousness.... For a well practiced operator, ... the control of sampling is usually unconscious, and driven by an intemal model of the environment. (Moray, 1980, p. 19)

In all likelihood the mental model is largely outside of consciousness, especially with regard to well-learned knowledge which controls display sampling, input-output relationships, decision making, and refined physical skills. Other authors have observed that, even if the model is partly accessible to conscious description, the reporting of nonverbal information (such the plan an operator uses to sample displays) may be difficult and distorted (Rouse \& Morris, 1986; Wickens, 1984). Therefore, approaches (such as in Whitfield, 1979) which rely solely on verbal data will not be effective in many situations.

In an attempt to guide investigative work, Rouse and Morris (1983) suggested that internal models of various types be defined by two dimensions, the "nature of model manipulation" and the "level of behavioural discretion". Their concept is diagrammed in Figure 3. 


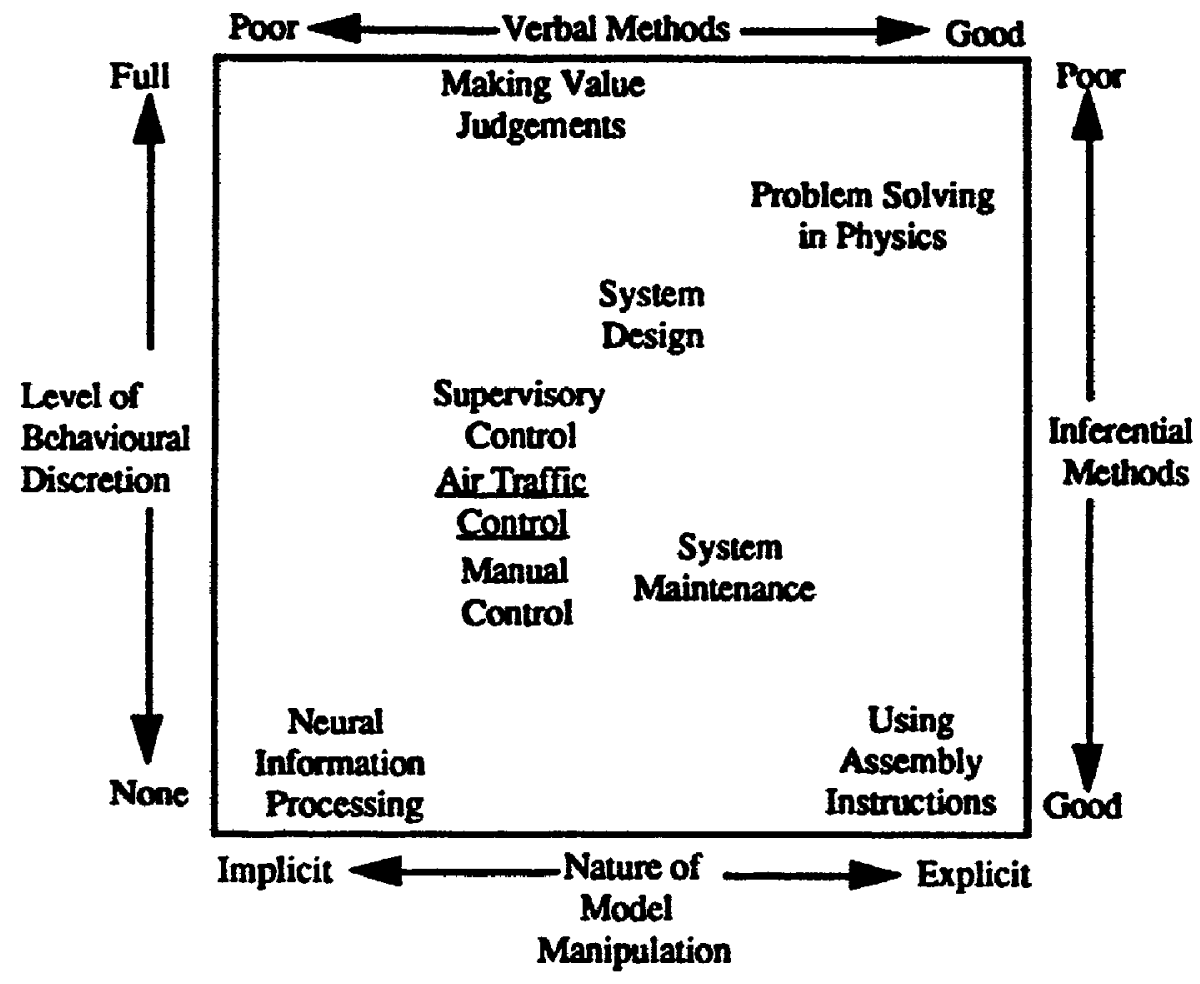

Eigure 3. Distinctions among domains of mental models. Note. Adapted from "On looking into the black box: Prospects and limits in the search for mental models" by W. B. Rouse and N. M. Morris, 1986, Psychological Bulletin, 100, p. 354. 
The nature of model manipulation axis refers to the degree to which the subject is aware of the use of an underlying mental model. For example, we are completely oblivious to how neuronal-level information processing proceeds in our brains, although it presumably has some kind of organization. However, other activities, such as using assembly directions, involve a more highly conscious use of procedures. The level of behavioural discretion axis describes the degree to which behaviour is open to choice or is dictated by a task demands.

Inferential methods, such as empirical and analytical modeling, are useful when the human is assumed to have little discretion. This is because the constraints of the system or domain restrict behaviour and make it feasible to infer mental operations from task descriptions and direct comparisons of input and output. Verbal methods will be effective when the domain involves higher levels of explicit manipulation because cognitive activities are more accessible to introspection. It can be seen from Figh -3 that situations represented in the upper left of the space will be difficult to study using any of these approaches because of the low level of self-awareness and high degree of choice.

Where would the activity of air traffic control lie in this chart? As a task, it is similar to supervisory control in that there is considerable monitoring of variables with the goal of error detection. However, there is also an ongoing requirement for intervention which introduces elements of manual control. Therefore, for the experienced controller, model manipulation may only be partially conscious with a range of levels of behavioural discretion, depending upon whether the operator is passively monitoring or actively controlling. According to the preceding guidelines, it should be possible to study air traffic control using both inferential and verbal methods. However, there will be limitations on the effectiveness of both techniques.

Rouse and Morris (1986) discussed the usefulness of discovering the forms, structures, and parameters of mental models. When researching the mental representations 
of the air traffic controller, it is important to consider the form (sensory code or spatial dimensionality ${ }^{1}$ ) the model takes, its structure or organization, and its parameters (amount of data, resiliency, or accuracy). These authors also noted that "expert's mental models are not just more elaborate or accurate; evidence suggests that they are fundamentally different from novice's models" (Rouse \& Morris, 1986, p. 356).

\subsection{Review of Studies on the Controller's Mental Model}

Research on the air traffic controller's mental model (or "picture"2) has been limited. Whitfield (1979) and Whitfield and Jackson (1982) elicited verbal reports of the picture from experienced air traffic controllers and suggested that such studies could assist with interface design, training, and selection. "Thus a better understanding of the picture should yield information on controller capacity based on the underlying psychological processes, rather than only on system measures" (Whitfield, 1979, p. 19).

In Whitfield's (1979) study, experienced controllers seemed to comprehend the picture in larger "units" (i.e. with more aircraft in each chunk). This statement is reminiscent of Chase and Simon's (1973) research on chess. They found that "the amount of information extracted from a briefly exposed position varies with playing strength" and that "the superior performance of stronger players (which does not appear in random positions) derives from the ability of those players to encode the position into larger perceptual chunks, each consisting of a familiar subconfiguration of pieces" (Chase \& Simon, 1973, p. 80).

Whitfield (1979) further commented that the air traffic controller's picture involves three different kinds of information in memory. A "static model" of the air traffic route

1 The use of "dimension" can be assumed to refer to the Cartesian spatial dimensions $(x, y$, and $z)$ unless otherwise specified. This word may also be used to refer to the dimensions in a multidimensional scaling solution which may or may not include the Cartesian spatial axes.

2 Air traffic controllers refer to their situation awareness as the "picture". Whether this is identical to or merely an aspect of the mental model will be discussed shorly. 
structures, separation guidelines, and procedures, a "dynamic model" including the changing attributes of particular aircraft (climb or descent rates, turns, etc.), and "working memory" for the current position and status of aircraft. He also observed that most subjects (especially controllers with non-radar experience) reported the picture to be a threedimensional, geographical representation which assisted with the understanding of the air traffic control situation. The identification of problems was also associated with the picture. It was sometimes described as a plan against which aircraft behaviour was compared and which helped in the detection of future conflicts.

Whitfield (1979) concluded that the picture assists the operator to feel in control, supplies information, and acts as a backup in case of equipment failure (performs as a "supplementary display"). However, beyond these general findings based on controllers" verbal reports, no further studies of the picture were conducted by these authors.

Lafon-Milon (1981) developed an empirical method to explore the spatial aspects of the air traffic controller's mental model. Expert and trainee controllers were shown eight static air traffic scenarios involving pairs of aircraft. One problem was a head-on approach, another was with overtaking aircraft, and six problems showed aircraft crossing tracks at 90 degrees. The controllers were asked to draw on paper the predicted relationship of the aircraft at the point of least separation. The resulting sketches were classified by whether they contained two-dimensional (plan or side view) or three-dimensional (plan and side view) information.

In the case of crossing track aircraft problems, it was found that $88 \%$ of the trainees drew a three-dimensional picture and only $12 \%$ drew a two-dimensional, plan view. On the other hand, $100 \%$ of the experienced controllers drew a plan view only. With regard to the head-on scenario. trainees generally produced a side view picture whereas experienced controllers drew a plan view; few from either group used a three-dimensional image. This same pattem was observed for the overtaking situations. 
When questioned, the trainee controllers emphasized the value of the altitude dimension $100 \%$ of the time whereas the experienced operators mentioned this in only $40 \%$ of the cases. As might be expected, the trainees were better at estimating vertical separation while the experienced controllers were more accurate with horizontal separation. It was concluded that the type of representation a subject produces depends on the experience of the controller as well as on the situation being presented.

An interesting experiment which used multidimensional scaling (MDS) to explore the spatial form of the controller's picture is "Spatial representation and the activity of the airtraffic controller" by E. A. Lapin (1982). This . uthor developed "a methodology for direct and indirect reconstruction of cognitive maps (using multidimensional scaling), as well as a diagnostic version of the methodology for studying mental rotation of three-dimensional objects" (Lapin, 1982, p. 3).

Two groups of air traffic controllers, highly qualified and satisfactory, were tested. Interobject distance judgements between targets on a radar display were processed using MDS and the results showed that the skilled group's stimulus space was three-dimensional while the less skilled group's space had two only dimensions. ${ }^{3}$ The additional dimension found for the expert controllers corresponded to "the normative profiles of losing and gaining altitude in the respective corridors" (Lapin, 1982, p. 3). When asked to reconstruct the air traffic situation, the more skilled controllers proceeded from global to local features while the "satisfactory" operators started from their local work area and then built up the general picture. The experts were also superior on a mental rotations test (adapted from Shepard, 1971).

Another research project on the cognitive aspects of ATC is by Landis, Silver, Jones, and Messick (1967). In their experiment 51 air traffic controllers were classified into four

3 This use of "dimension" here refers to MDS dimensions which may represent any variable (spatial or otherwise) which explains a proportion of the variance in a set of comparison judgements. 
groups based on job title. Within three of these groups, the controllers were further subdivided into levels of competence. Thirty air traffic control problems were drawn on 8 X 10 inch map overlays of an air traffic sector (see Figure 4). All possible pairs of stimuli were printed on 435 cards which the subjects sorted into 16 categories based on similarity. Using an early MDS procedure, it was determined that at least four "points of view" were being employed. The pool of all subjects was then classified into four subgroups based on their loadings on these viewpoints. Landis et al. (1967) observed that the correspondence of training and competence to viewpoint group was "striking". For example, the subjects assigned to Viewpoint Group $\mathrm{C}$ were a skilled group while, on the other end of the continuum, members of Viewpoint Group A were relatively unskilled. Groups B and D appeared to represent intermediate competence levels.

The next step in the data analysis was to average the similarity ratings of the subjects within each viewpoint group and then proceed with an MDS solution for an "idealized" or average individual for each group. The number of salient MDS dimensions for each viewpoint group ranged from one to three. The original air traffic control problems were then ranked according to their factor loadings on each of these dimensions. Air traffic control instructors examined the problems and the following "hypotheses" were suggested for the collective dimensions:

1. amount of watching

2. number of aircraft

3. number of directions

4. amount of analysis required

5. severity of problem

6. necessity for action 


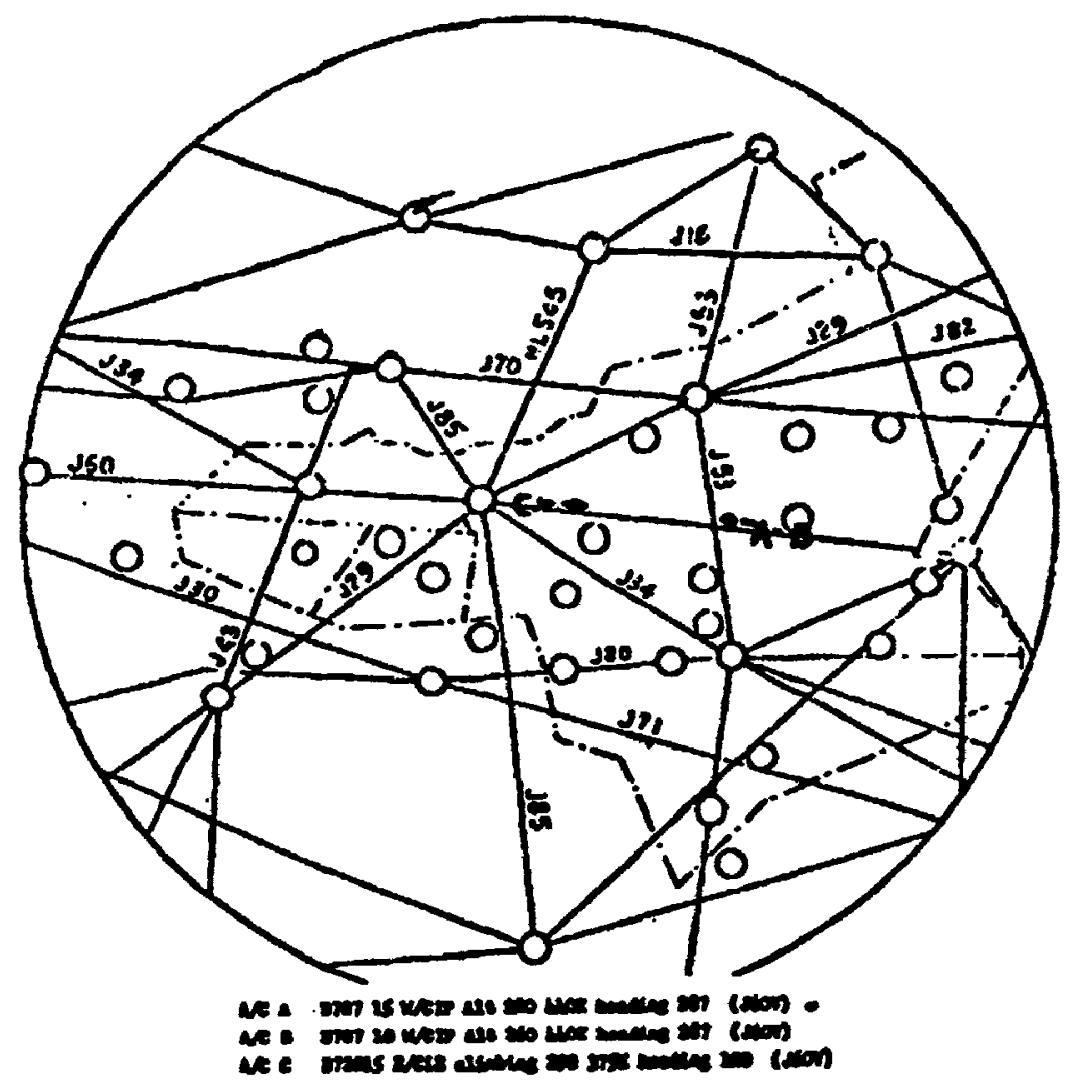

Eigure 4. A sample stimulus from the Landis, et al. (1967) study. Note. From "Level of proficiency and multidimensional viewpoints about problem similarity" by D. Landis, C. A. Silver, J. M. Jones, and S. Messick, 1967, Loumal of Applied Psychology, 51, p. 217. 
It was found that subjects in Viewpoint $A$ (least skilled) used different dimensions to judge problem similarity as compared to the skilled controller group (Viewpoint Group C).

Skilled radar controllers differentiate among air-traffic control problems more in terms of varying operational responses they require than in terms of the stimulus characteristics per se. .... The judgements of the least skilled group (Viewpoint A) appeared to be based upon many simple dimensions depicting various stimulus characteristics, whereas the judgements of the most skilled group appeared to be based upon a small number of complex dimensions reflecting the response requirements of the stimulus situations. (Landis et al., 1967, p 221)

This research indicates that there is more to the controller's internal model than merely a visuospatial mental image; there are task-related rules which influence the controller's understanding of the information available from the system. The complexity of these rules varies with controller expertise.

A more comprehensive project on the cognitive aspects of air traffic control was done by Means, Mumaw, Roth, Schlager, McWilliams, Gagne, Rice, Rosenthal, and Heon (1988) in which they developed a "cognitive task analysis". Three highly skilled enroute conirollers were presented with three dynamic traffic simulations. Two of these employed radiu and the third was "manual" (or procedural) using flight data strips only. In each condition, subjects were first asked to recall as many aircraft (and their attributes) as possible on a blank sector map and then to draw circles around groups of aircraft which had some common characteristic. As part of the same recall task, they also drew significant "events" from the simulation on another map. Subjects were also asked to fill in blank flight data strips for selected aircraft from each simulation exercise and to attempt problems requiring conflict prediction.

In general, controllers were able to correctly recall $86 \%$ of the aircraft at the end of the simulation exercises (i.e., $86 \%$ of the aircraft recalled could in some way be identified as actual targets). Table 2 shows the results for specific aircraft attributes. With regard to the grouping data from this task, each cluster contained two to three aircraft which were 


\begin{tabular}{|l|c|}
\hline \multicolumn{1}{|c|}{ VARIABLE } & \% CORRECT \\
\hline Position & 72 \\
\hline Identifier & 43 \\
\hline *Altiude & 23 \\
\hline *Heading & 16 \\
\hline
\end{tabular}

Table 2. Per cent of aircraft attributes correctly recalled. (*The recall of altitude and heading were not requested but were volunteered by some subjects.) Note. From ATC Training and Analysis Study: Design of the Nexl-Generation ATC Trainine System (p. 106) by B. Means, et al., 1988, Richmond, VA: HumRRO International. 
characterized by a specific kind of air traffic control problem, such as arrivals or aircraft on crossing tracks.

There was a relationship between the way aircraft were grouped in the recall task and the order of recall. In nearly all cases, all of the aircraft from one group were remembered before any of the aircraft belonging to a second group. The authors suggested that this was evidence that the controller's grouping of aircraft guided the retrieval of screen information from memory. There were also indications that groups of aircraft were not merely organized on the basis of two-dimensional spatial proximity but as determined by taskrelated characteristics.

When subjects were asked to recall significant events from a scenario, they identified four to eight groups with two to four aircraft per group. Groups were again characterized by particular air traffic control problems in which separation had to be applied.

Controllers, if they recalled a specific group, always remembered all of the aircraft from the radar screen which would be included in it.

In the blank flight strip recall task, the experimenters categorized six aircraft from earh simulation with regard to the amount of control the subject exercised over them. Three were classified as requiring considerable attention and three as needing little consideration. After the traffic drawing tasks for each exercise were completed, the subjects were given six blank flight data strips and asked to recall as much information as possible for each of the high and low attention aircraft.

Subjects' overall recall of aircraft attributes is shown in Table 3. An emphasis on position information and altitude can be observed. High attention aircraft tended to be recalled more often in the prior recall tasks and accounted for $38 \%$ of the flight strip information remembered (as opposed to $17 \%$ for the low attention aircraft). Subjects recollected data which was related to the type of control exercised over an aircraft. For 


\begin{tabular}{|l|c|}
\hline \multicolumn{1}{|c|}{ VARIABLE } & \% CORRECT \\
\hline Posted Fix & 46 \\
\hline Routing & 46 \\
\hline Altimde & 41 \\
\hline Control Data & 39 \\
\hline Requested Altitude & 30 \\
\hline Type & 28 \\
\hline Arr.Dep. Arrows & 19 \\
\hline Next Fix & 7 \\
\hline Ground Speed & 6 \\
\hline Transfer Data & 0 \\
\hline
\end{tabular}

Table 3. Per cent of aircraft attributes correctly recalled from the blank flight strip task. Note. Erom ATC Trainine and Analysis Study: Desion of the Next-Genemtion ATC Training System (p. 106) by B. Means, et al., 1988, Richmond, VA: HumRRO Intemational. 
example, routing into the sector was recalled much more frequently for aircraft which had been vectored. 4

Two prediction tasks were also included in this study. One was to plan aloud from a set of flight strips (procedural or non-radar control) and the other was to plan aloud from a scenario already in progress, using a radar screen, flight strips, and a verbal briefing from another controller.

In order to investigate how far into the future controllers could detect possible conflicts, six aircraft were added to the end of the non-radar and radar situations and arranged so that they would become traffic for the existing aircraft 10,30 or 50 minutes in the future. It was found that controllers in the non-radar condition were able to look ahead about 29 minutes on the average, over twice as far as when radar was used.

This can be explained in part because of the demands in procedural control for greater separation standards than those required with radar, it becomes necessary to think further ahead due to these rules. The authors commented that:

Although the majority of operational controllers do not normally control traffic in a nonradar environment, habits of planning ahead and the ability to visualize traffic in three dimensions are regarded as standards of good controller practice, and it is the belief of many controllers that these skills are best developed through practice in the nonradar environment. (Means, et al., 1988, p. 95)

There have been other researchers who used a recall task in studies of air traffic control. Bisseret (1970) asked three groups of subjects (highly qualified controllers, average controllers, and trainees) to recall seven aircraft atuributes 5 for se's of five, eight, or eleven targets. After a period of work with a dynamic simulation, subjects were asked to recall aircraft in three different ways. First, a sector map was provided on which they were

\footnotetext{
4 "Vectoring" an aircraft involves giving course, speed, and altitude change commands in order to maintain separation and to bring the aircraft to its planned fix or runway. "Routing" refers to the series of navigational fixes an aircraft is supposed to cross over in its flight path.

5 Aircraft identifier, type, altitude (and climb/descent), position, time, previous track, and projected track.
} 
to record as many aircraft as possible. Next, on a blank page, subjects were asked to recall as many aircraft attributes as they could from the simulation. Finally, the experimenter described the characteristics of new aircraft entering the situation and asked for conflict predictions.

Some of Bisseret's (1970) results are shown in Tables 4, 5, and 6. It is clear that the most highly qualified controllers had the best recall abilities. However, there does not appear to be much difference between average controllers and trainees. Bisseret (1970) found that there was a definite ranking of attributes across groups of subjects and workloads. As seen in Table 6, altitude and position information were remembered most frequently. This is similar to the data found in Means, et al. (1988), (see Table 3).

Moray (1980) also examined the role of memory in air traffic control. He described the limitation of short term memory to about eight items and of running memory to about three items. "We may conclude that holding even very simple information in memory for subsequent use, while simultaneously entering new information and removing old information, is extremely difficult for humans" (Moray, 1980, p. 32).

He also reported that the results of research on the rate of forgetting of a simulated radar echo could be expressed in the form of an equation:

$$
\left.S D_{\mathfrak{l}}=g+0.02(t)\right)^{1.5}
$$

where $g$ is a constant whose value depends on the experience of the controller, ${ }^{7} t$ is the time since an observation was made, and SD is the standard deviation of the estimate of the position of the echo. The value of $g$ also depends upon the number of echoes, and other properties of the display, and is in the range $5-15$. (Moray, 1980, p. 57)

6 "Running memory" refers to the ability to retain information in short term memory while attending to new information which will have to be recalled. One test of running memory is to verbally present a series of digits, asking the subject to continually repeat back the number given a few steps previous to the immediate number being said. Running memory will also be called "working memory" in this paper. unler coted otherwise.

7 It is istumed that " $g$ " is inversely related to ATC experience, although Moray (1980) was not specific about this. 


\begin{tabular}{|l|c|c|c|}
\cline { 2 - 4 } \multicolumn{1}{c|}{} & \multicolumn{3}{c|}{ NUMBER OF AIRCRAFT } \\
\hline GROUP & 5 & 8 & 11 \\
\hline Expen Controllers & 0 & 10.0 & 10.4 \\
\hline Averaze Controllers & 0 & 12.5 & 43.6 \\
\hline Trainees & 4.0 & 32.5 & 38.2 \\
\hline
\end{tabular}

Table 4. Per cent of aireraft not recalled in the map condition. Note. From "Memoire operationelle et structure du travail" by A. Bisseret, 1970, Bullerin de Psychologie. 24, p. 286.

\begin{tabular}{|l|c|c|c|}
\cline { 2 - 4 } \multicolumn{1}{c|}{} & \multicolumn{3}{c|}{ NUMBER OF AIRCRAFT } \\
\hline GROUP & 5 & 8 & 11 \\
\hline Expert Controllers & 0 & 5.0 & 12.7 \\
\hline Avcrage Controllers & 0 & 10.0 & 25.5 \\
\hline Trainces & 4.0 & 22.5 & 36.4 \\
\hline
\end{tabular}

Table 5. Per cent of aireraft not recalled in the blank page condition. Note. From "Memoire operationelle et structure du travail" by A. Bisseret, 1970. Bulletin de Psychologie, 24, p. 286.

\begin{tabular}{|l|c|}
\hline \multicolumn{1}{|c|}{ ATTRIBUTE } & NO. RECALLED \\
\hline Alinude & 296 \\
\hline Position & 277 \\
\hline Time & 127 \\
\hline Identifie & 65 \\
\hline Type & 57 \\
\hline Future Track & 52 \\
\hline Previous Track & 25 \\
\hline
\end{tabular}

Table 6. Number of aircraft attributes correctly recalled. Note. From "Mémoire operationelle et structure du travail" by $A$. Bisseret, 1970. Bulletin de Psychologie, 24. p. 286. 


\subsection{Evaluation of Previous Research}

The preceding review of research on air traffic control suggests an international concern with the mental processes of the air traffic controller. Although references to mental models are not always explicitly stated, all of the findings from these studies provide information on the form, structure, and parameters of the mental model of the air traffic controller.

With regard to the form of the controller's picture, Whitfield (1979) reported that many controllers described a three-dimensional, geographical image. Lafon-Milon (1981) investigated this by analyzing trainee and experienced controller's drawings of various air traffic problems. She found that the only time a complete three-dimensional representation (plan and side views) was offered was by trainees in response to crossing tracks problems. Experienced controllers drew all of the problems in a plan view suggesting that the tendency to employ three-dimensional spatial visualization diminishes with increasing time in the job.

However, this conclusion is thrown into doubt by Lapin's (1982) work. He asked two groups of controllers, highly qualified and satisfactory, to make distance judgements between objects in a radar display. The more skilled subjects appeared to be using a third MDS dimension related to altitude which was lacking in the two-dimensional solutions of the other group of controllers.

Without knowing more about the methodology used in Lapin's (1982) study, it is difficult to explain why his results differed from those of Lafon-Milon (1981). It may be that the demand characteristics of his experiment prompted the controllers to include the factor of altitude in their distance estimates even though they did not normally visualize in three spatial dimensions. Lapin's (1982) findings suggest that skilled controllers are able (under his experimental conditions) to employ the third dimension in their judgements but this is not conclusive proof that they usually do so. On the other hand, Lafon-Milon's 
(1981) observations that experienced subjects drew only two-dimensional views of air traffic problems does not rule out that they used three-dimensional imagery to solve these problems. Her experienced subjects may have merely found the customary display format (plan view) the most natural means for representing their solutions.

This still leaves open the question of whether the controlier's picture is an internal spatial representation in three dimensions as suggested by Whitfieid (1979), or whether it is only two-dimensional, or even lacking in any visuospatial qualities. In air traffic control, a primary source of information for the creation of a mental image is aircraft data available on the radar screen. In the case of the Canadian Radar Data Processing System the $x, y$ location of an aircraft is pictorially represented while altitude is displayed numerically (see Figure 5).

Rather than adding a third spatial dimension with the altitude data available at the interface, the controller may only employ quantitative altitude information to support the required judgements. For example, according to air traffic control procedures, if two aircraft are separated by more than 1,000 feet in altitude, a conflict situation does not exist. Rather than creating an internal spatial image to compare altitudes, the controller may merely consult the altitude values shown on the radar screen and perform a simple subtraction to find out if the altitudes of two targets differ by more than 1,000 feet. Thus the picture, if any, may only contain a mixture of spatial and quantitative information which supports (largely unconscious) rule-based comparisons.

The work by Lapin (1982) and Lafon-Milon (1981) on the form of the internal representation of air traffic demonstrates the problems inherent in uncovering the actual mental imagery employed by the controller. It is extremely difficult to design an experiment in which demand characteristics, task requirements, or display formats could not be claimed to be influencing a subject's responses. 


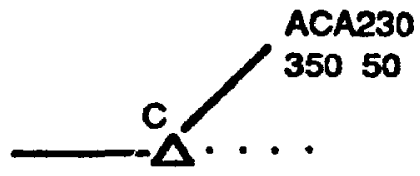

Eigure 5. The data block as displayed on an air traffic control radar screen. Aircraft location is denoted by the triangle, previous track by the dots, and planned track by the solid line. "ACA230" is the identifier (or flight number), "350" is altitude (X100), and " 50 " is speed (X10). The letter near the triangle indicates which controller is assigned to monitor the aircraft. 
It is also evident from these studies that skill and/or training affects the kind of spatial imagery used by controllers. An interesting observation put forward by Means, et al. (1988) is that such abilities as planning and the visualization of traffic in three dimensions may be related to the amount of time a controller has had in a non-radar environment. Deprived of the assistance of a visual display of radar data and supplied only with flight data strips and voice communications with aircraft, it is easy to assume that special mental skills would be developed in this situation to visualize the present and future positions of aircraft. Therefore, the way a controller organizes, remembers, imagines, and predicts may not only be based on amount of experience and skill attained, but also on the display environment.

When considering the organization and parameters of the controller's mental model, the research employing recall procedures is very informative. Whitfield (1979) emphasized the role of memory in the creation of the picture. Aithough he did not supply empirical evidence, he also suggested that controllers use chunking to organize traffic on the radar screen. This was supported in the experiments by Means, et al. (1988) in which controllers organized the radar display into groups of two or three aircraft and tended to recall all of the aircraft in a certain group.

Given Moray's (1980) reminder about the limitations of short term memory, some type of strategy (such as chunking) must be operative to explain the ability of subjects to recall as much information as reported in Bisseret (1970) and Means, et al. (1988) These researchers also discovered that their subjects favoured the recall of altitude and position information over other aircraft attributes. ${ }^{8}$ In addition, the variables remembered may depend upon the type and amount of control being exercised over the target (Means, et al., 1988).

8 It is assumed here that frequency of recall of a particular aircraft attribute (such as altitude) is directly related to its importance to the subject. 
It is easy to see how each aircraft icon with its associated attributes could be considered as a unit or chunk. If understood as a real aircraft flying at a speed, heading, and altitude planned to carry it to a certain destination, it can be seen how the variables could be meaningfully interrelated. Beyond this, as discovered by Chase and Simon (1973) with chess pieces, a nuriter of aircraft in a particular problem configuration would form a meaningful pattern which could become a larger chunk. If this is the case, more knowledgeable controllers would remember more (as found by Bisseret, 1970, and Moray, 1980). Their training and experience should cause them to have a greater ability to meaningfully group the stimuli.

The work of Landis, et al. (1967), although not using a recall paradigm, supports this notion. These authors demonstrated that the degree of complexity with which a controller views air traffic control problems increases with expertise. ATC skill level influences whether an air traffic scenario is classified by straightforward characteristics, such as number of aircraft, or by more sophisticated, task-related factors. These findings, com:ined with those from the recall studies, suggest that specific organizational forces are at work in the controller's mental model anci provides indications of the model's capacity and accuracy.

The controller's mental model possesses various kinds of information which are reliant on different types of memory. Maps, flight plans, aircraft nerf'rmance information, separation standards, and procedures are learned through training anc experience and stored in long term memory. It is this knowledge which imposes structure and guides the chunking process. However, as Moray (1980) and others have suggested, this part of the controller's mental model, once learned, may be largely unconscious.

The term "picture" (as used in the profession) refers to the conscious parts of the internal model, such as could be maintained in short term memory. It may be true that the non-conscious aspects of the model control sampling and organization, but the influence of 
this underlying learning is reflected in observable activities such as the kinds of information sampled, how it is organized, and what is selected for short term storage. For example, Means, et al. (1988) observed a traffic drawing task and discovered that ATCs segregate aircraft into groups. The unconscious rules guiding this patterning behaviour were inferred from an analysis of the clusters.

Also probably present in short term memory are records of recent verbal communications, projected flight paths, problems to be addressed, and plans for activities in the next few minutes. Therefore, a method for exploring the unconscious parts of the the controller's mental model may be to map out the conscious picture which is developed from interaction with the moment-to-moment events at the radar interface.

\subsection{Outline of Thesis Research}

Given the limited amount of research in the area of mental models, it was decided to conduct a Preliminary Study to test a number of empirical approaches for possible refinement in further experiments. The results of this work are summarized in the next section and the details of the Preliminary Study are provided in Appendix A. A number of interesting findings were obtained from these studies and two further experiments were designed to demonstrate the importance of the controller's picture. These are described in Chapters 3 and 4. Finally, the information gained from the Preliminary Study and the experiments is summarized and discus:ed in Chapter 5. A comprehensive set of appendices contains supporting information for the Preliminary Study and Experiments 1 and 2.

The research described here addresses both theoretical and applied questions. The Preliminary Study was designed to pursue a number of the theoretical issues raised in the introduction. Experiments 1 and 2 were intended to demonstrate a theoretical point but were also planned with practical goals in mind. The theoretical and applied themes of this research are intermingled in Chapters 3 and 4 but are separated for discussion in Chapter 5 . 


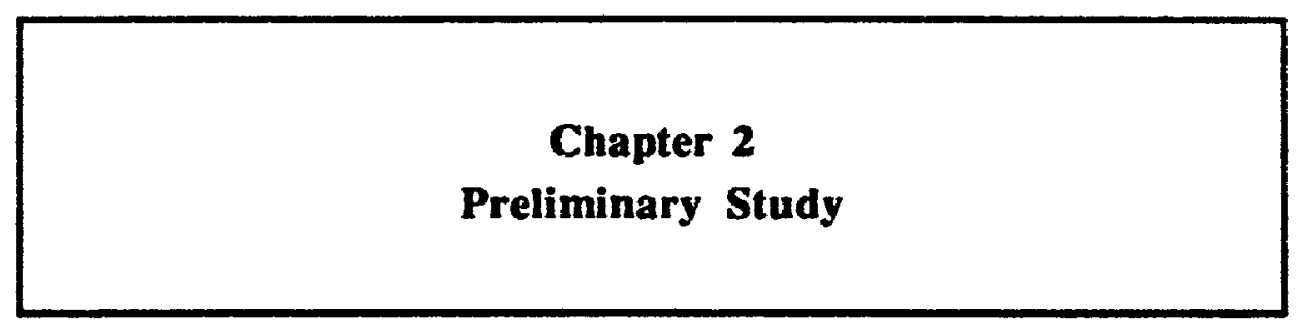


A summary of the Preliminary Study carried out at the Transport Canam Research and Experimentation Centre is included here in order to outline important findings and to provide a basis for Experiments 1 and 2 which follow. A more extensive rationale, description of the experimental method, and report of findings are found in Appendix A.

\subsection{General Approach}

As mentioned previously, mental models can be considered in terms of their forms, structures, and parameters (Rouse and Morris, 1986). The Preliminary Study incorporated a number of ways of exploring the form of the air traffic controller's picture (such as its sensory code or spatial dimensions), how information is organized or structured, and its characteristics (amount of data, resiliency, or accuracy). In addition, because one of the purposes of a mental model is to help predict the behaviour of a system, assessments of controllers' ability to project the future positions of aircraft and to detect unexpected changes in their movement were also included.

Based on Whitfield's (1979) analysis, it was assumed that the controller's picture is held in short term memory and periodically updated from visual scanning of the screen and from verbal communication with aircraft. A realistic simulation was thought to be the best vehicle for properly exploring the picture.

As Norman (1986) pointed out earlier, interaction with a system is required in order to develop a good User Model. It would be unrealistic to expect a controller to understand the the dynamics of an ATC system without having any experience with it. If a static simulation were employed the range of skills which subjects could apply would be artificially restricted.

An air traffic control simulation program called TRACON (used by NASA and other facilities) was chosen for the Preliminary Study (Wesson \& Young, 1988). A special radar map background was designed, a series of flight plans was created, and the experiments were run on an IBM-PC with multiple monitors. Experimental exercises and subject 
responses were recorded on videotape. The form, structure, and parameters of the controller's picture and its ability to support prediction were addressed by applying the experimental conditions shown in Table 7. The experiments were designed to identify the unique picture-related skills of experienced controllers as compared to non-controllers.

Subjects were recruited from three sources. The most readily accessible personnel were those working as "pilots" (operating the simulator) at the Transport Canada Air Traffic Services Research and Experimentation Centre. Four participated (three females, one male; average age=28). Experienced controllers were more difficult to find. Two retirec controllers, one controller who was being retrained, and two instructors from the Transport Canada Training Institute were available (five males, average age of group=51). In addition, three undergraduate students from Carleton University (two females, one male; average age $=22$ ) were sought out given their presumed lack of knowledge about air traffic control.

\subsection{Results}

\subsubsection{Recall Order}

There were a number of possible factors operating apart from task relevance which might have affected order of recall of aircraft from the radar screen. Nevertheless, the Preliminary Study demonstrated that elements of a subject's picture have a task-related valence in memory which improves the probability that they will be given priority of recall.

This was seen in two ways. First, from analysis of the radar situation at the time of the recall task, it was evident that subjects tended to remember most frequently the aircraft which were on the final stages of approach or which would soon otherwise require attention. Secondly, subjects' frequency of recall correlated significantly with their own rankings of the importance of the aircraft and with rankings by independent judges (controllers not involved in the study). 


\begin{tabular}{|l|l|}
\hline \multicolumn{1}{|c|}{ EXPERIMENTAL TASKS } & \multicolumn{1}{|c|}{ OUTCOMES } \\
\hline Recall of Screen Targets & $\begin{array}{l}\text { Odda } \\
\text { Rate } \\
\text { Accuracy }\end{array}$ \\
\hline Target Similarity Judgements & Grouping of Targets \\
\hline $\begin{array}{l}\text { 3D Spatial Judgements: } \\
\text { (a) Interobject Distance } \\
\text { (b) Height of Ceiling } \\
\text { (c) Present and Predicted Positions }\end{array}$ & 2D or 3D Distance Correlations \\
& $\begin{array}{l}\text { Altitude Expansion Factor } \\
\text { Response Time } \\
\text { References to Screen } \\
\text { Accuracy }\end{array}$ \\
\hline Fault Detection & Number Detected \\
& Response Time \\
\hline
\end{tabular}

Table 7. The experimental conditions included in the Preliminary Study. 


\subsubsection{Recall Rate and Accuracy}

Although between-subjec : variance in speed of recall mitigated against the appearance of clear between-group differences, it was shown that three of the controllers were faster than non-controllers. However, two of the controllers were as slow or slower than the non-controllers. In one case, this seemed to be due to generally poor recall abilities. Another controller took an excessive amount of time to draw the last target remembered and this artificially reduced the recall rate.

Controllers had more accurate recall of target position as measured by absolute and ballpark error methods. ${ }^{9}$ Bisseret (1970) and Moray (1980) also found that highly skilled controllers remember aircraft information better. Controller group performance was also more consistent. ${ }^{10}$ These results are especially impressive given that the controllers tested were on the average 29 years older than the students and 23 years older than the pilots. If some decline in faculties due to age can be assumed the recall abilities of younger controllers could be expected to surpass those of non-controllers to an even larger degree.

However, a few non-controllers were also accurate with recall. This result suggests that the ability to form a good representation of air traffic in short term memory (or a good picture) may be aptitude-based rather than exclusively generatsd by training or experience. If good performance were restricted to the controller group alone this premise would not be warranted.

It is not surprising that some non-controllers might possess this ability. It could be assumed that those in the controller group were selected for ATC work based in part on the possession of good memory or chunking ability. These results suggest that recall

\footnotetext{
${ }^{9}$ Differences between controlters and non-controllers were significant for three-dimensional absolute and ballpark accuracy (see Appendix C-4.2).

10 See Appendix C-4.3.A for ANOVA data.
} 
efficiency (quantity and rate) and accuracy may be good measures of the aptitude for forming and using the air traffic controller's picture.

Another interesting finding was that subjects remembered certain aircraft attributes better than others. The data for the controller group are similar to Bisseret (1970) and Means, et al. (1988) in that their subjects also emphasized altitude and position information over other aircraft attributes.

\subsubsection{Target Organization}

The expectation that controllers would have a unique and more refined system for grouping aircraft was not confirmed in the cluster analysis results. Mnst subjects organized the nine aircraft on the TRACON monitor into five groups. Contrsllers were more variable than the non-controllers employing from two to six groups. Upon review of the kinds of distinctions made between aircraft, the controllers often employed the same rules as other subjects for forming clusters and in two cases indicated a very simplified structure placing the aircraft into only two groups (arrivals and overflights).

An unexpected finding was that controllers made a greater number of extreme similarity judgements (i.e., that targets were either completely different or exactly the same). This is an interesting demonstration of a "dichotomizing" controller decisionmaking style often reported to the experimenter by controllers and noted elsewhere in the ATC literature (Bisseret, 1981). There was little indication that clusters of aircraft were recalled together as in Means, et al. (1988).

\subsubsection{Spatial Tasks}

When subjects' interobject distance judgements were correlated with actual aircraft separation the expectation that controllers would show evidence of three-dimensional spatial imagery was partially met. Two out of five controllers employed altitude in their judgements. However, two of the student subjects also saw the TRACON display in this way. 
A series of plotting tasks was included as another way to examine three-dimensional spatial skills." However, controllers did not show convincing signs of unique or exceptional performance. When making altitude estimates over a sector map their results were similar to those of the other subjects indicating that there is a tendency to expand the vertical scale. The amplification was from 9.7 to 18.5 times what would have been expected with reference to the horizontal scale of the map. A test of this effect before the experiment as well as after some time controlling aircraft might have been more effective in showing the preexistence and/or development of this perception.

Controllers did not show better accuracy in placing the slider on a position and altitude plotting marker to various altitudes during a practice session. ${ }^{12}$ There was a hint of faster completion of plotting exercises and somewhat more consistent and accurate results in plotting present aircraft position ("model building") on the plotting board. However, two minute predictions of position were no better than for non-controllers. Five minute predictions were somewhat better than for other subjects if one controller's results can be ignored. There was evidence that controllers checked the screen less often while completing plotting tasks (about every nine seconds)..$^{13}$

It was hoped that plotting the present and future positions of potentially conflicting aircraft would call up exceptional spatial abilities. However, there was no evidence of this. In general, non-conflicting aircraft were plotted with the same accuracy as conflicting targets.

However, there was an indication that controllers performed more accurately than naive subjects when predicting the two minute positions of arriving as opposed to overflying aircraft. This is in line with the expectation that training and experience might play a role in

11 Subjects were asked to plot three-dimensional present and predicted aircraft position on a plotting board using a sector map and knitting needles ("markers") with vertically adjustable beads ("sliders").

12 See Appendix A, Section A-2. for a description of the method.

13 This was assumed to be a measure of the resiliency of the subject's mental image of the screen. 
some tasks. Overflying aircraft in the exercise followed straight-line routes at constant speeds. In contrast, subjects had to form a plan for an arriving aircraft and follow it.

Moray (1980) noted that when controllers have an aircraft under supervision, its future position is more predictable than when the aircraft is under a pilot's control; in the latter case there is more uncertainty as to what the pilot will do. This could make the monitoring of overflights more difficult than the control of arrivals.

The lack of clear between-group differences in the spatial data may indicate that the tasks were not difficult enough. It is also possible that only rudimentary spatial imagery is required in air traffic control or that the tasks designed for this experiment lacked construct validity and did not tap the kinds of skills which are important.

\subsubsection{Fault Detection}

Six of the subjects detected most of the pilot errors made by the experimenter in a relatively short time. However, only two were controllers. It was expected that more of the controllers would do well at this task.

However, five of the six vigilant subjects also performed well on the recall task. Error detection probably relies on the visual sampling of targets on a regular basis and the comparison of the perceived attributes with those in memory (or in the picture). Thus, it is not surprising that those with good recall ability also detected faults well. It may be that the subjects who had good a picture but who performed poorly on fault detection were not making the necessary comparisons for some reason. Perhaps their attention was completely captured by the primary task of controlling aircraft in an unfamiliar airspace using a novel radar display.

\subsection{Conclusions}

The preliminary research was intended as a means of identifying specific areas for further study. Due to the small number of subjects, the results must be considered as tentative. The recall data showed the clearest indication of between-group differences. The 
suggestion that good recall ability is aptitude-based also emerged from these findings. Although a few non-controllers remembered aircraft well, the controller group showed more consistent and accurate performance. There was a limited indication of betweengroup effects in the modelling and prediction data but, perhaps due to the simplicity of the tasks and small number of subjects, there was not as clear a trend as in the recall results.

There were some uther interesting qualitative findings from the Preliminary Study. These included evidence for a task-related prioritization of targets in short term memory, indication of three-dimensional spatial imagery, the existence of an altitude expansion factor in spatial judgements, and the demonstration of a dichotomizing decision-making style on the part of controllers. Each of these themes could be explored further on its own. However, in the context of defining the air traffic controller's mental model, additional research employing the recall of sereen targets as a measure of the controller's picture holds the most promise.

\subsection{Rationale for Further Experiments}

The Preliminary Study included a varizty of experiments designed to explore the air traffic controller's mental model. There were a number of interesting results which will support a limited description of the operator's picture and these will be elaborated upon in Chapter 5.

One of the prominent findings in the Preliminary Study was that controllers had more accurate recall for screen information than naive subjects and that they preferred specific aircraft information (altitude and position over identifier and speed). This not only suggests that controllers possess exceptional memory abilities but that these skills are employed for a specific purpose; to retain certain kinds of task-related information.

Whitfield's (1979) work supports the assumption that aircraft attributes are components of the picture and there is no doubt that air traffic controllers subjectively experience it. This information is present in the moment-to-moment awareness of the 
controller. Within the constraints proposed by Rouse and Morris (1986) and Ericsson and Simon (1980) it should be possible to access the controller's thoughts by asking for descriptions of air traffic during an interruption of simulated work. Some of the limitations of purely verbal accounts may be overcome by asking for location plots as well as for alphanumerical aircraft data.

The role of memory in maintaining the picture was mentioned in the introduction under the discussion of Whitfield's (1979) study. He noted that "It seems likely that some items of the picture are carried in transient memory, and some of the controllers' comments support this" (p. 25) and described the three kinds of memory which are probably involved (static, dynamic, and working).

There is further mention in the literature of the use of a memory paradigm for studying complex visual tasks. The studies of chunking in chess by de Groot (1965) and Chase and Simon (1973) used a recall paradigm. Pinker and Finke (1980), in their research on three-dimensional imagery (discussed in Chapter 4) asked subjects to memorize and then visualize an array of objects. Bisseret (1970) and Means, et al. (1988) employed recall tasks in their ATC work.

The role of memory in air traffic control is further illuminated by Sperandio (1978):

The operational (or 'working') memory, defined by Bisseret (1970) as: 'a temporary memory of real data, organised and structured by the processes of work', exhibits a rather clear division between useful information (always retained in memory) and information which has become useless (which is retained only within the limits of 'available space' in the memory); priority always being given to storage of information which remains useful for dealing with current work. [italics added] (p. 198)

Vincente (1988) presented a convincing argument for the use of a memory recall approach for exploring mental models of systems. He reviewed the studies on chess and other research which demonstrated that chunking relies on the perceived meaningfulness of the stimuli in question. Meaningfulness in a given domain, he argued, is dependent on knowledge. He concluded that a good measure of an expert's mental model would then be 
to ask for the recall of information about the system. A subject would presumably divulge what is meaningful (i.e. what had been chunked in memory) and would thus provide a glimpse of their internal representation.

The identification of a technique to measure some aspects of the picture raised the possibility that the next phase of this research could go beyond describing the picture to demonstrating whether it is practically useful. Although many statements have been made about the presumed value of the picture and of mental models in general (Whitfield, 1979; Wickens, 1984) there has been no empirical demonstration of this. However, by using a recall task to assess the picture it is recognized that the emphasis is placed on what is recalled rather than what is present. The resulting view of the controller's picture will be of elements which are being retained for some reason. Therefore, the present 2 esearch is by definition focused on how useful the remembered elements of the picture are in the exercise of air traffic control. Information which is in momentary awareness and is not stored is not accessible using this approach.

In addition to adapting the recall procedure from the Preliminary Study to measure the accuracy of the picture in further experiments, it was necessary to collect other kinds of information. In order to establish the importance of the picture for good air traffic control, controller skill had to be assessed in some way. Although this was a difficult to address at the R. \& E. Centre due to a lack of acceptable criteria it was found that both academic and practical measures of expertise were collected on a regular basis at the air traffic control school. This fact, as well as the availability of a large group of subjects, indicated that research on the importance of the picture would be best carried out at the Transport Canada Training Institute (TCTI).

It was also decided to include other tests in the project. In order to discriminate whether controller trainees just had superior memory abilities in general as opposed to specific skills for retaining the picture, three subtests from the Leaming and Memory 
Battery (LAMB) involving different aspects of short term memory were given (Tombaugh \& Schmidi, 1989). It was assumed that if this testing revealed that good general memory aptitude was not as good a predictor of skill as the recall of aircraft information, the importance of the picture would be clearer.

The following two experiments had two main purposes. One was to test the importance of the picture in air traffic control. This inciuded determining whether the differences between the radar-based and procedural (non-radar) work environments had any effect on the kinds of information in the picture. There was also an applicationsoriented theme. It was hoped that a number of variables would emerge in each experiment which would prove useful in predicting trainee success in the academic and practical aspects of air traffic control training. Therefore, the Differential Aptitude Test (DAT) (Bennett, Seashore, \& Wesman, 1988), an accepted instrument in vocational counselling, was chosen to gather other kinds of information about the subjects. 


\section{Chapter 3}

\section{Experiment 1: Radar Environment}




\subsection{Introduction}

Experiment 1 assessed the importance of the controller's picture in the radar environment. It was decided that the relationship of picture quality to controller skill could best be answered by focusing on ATC trainees. The chances for demonstrating the relationships of interest were superior due to the availability of methods for assessing ATC trainee proficiency, the presence at TCTI at any give time of over 100 subjects in various stages of training, and the existence of a more homogeneous subject group with regard to experience and age.

However, equipment and staff time were even more limited at the school than at the R. \& E. Centre and it was not possible to nun a cor trol group of non-controllers against which to compare picture accuracy. Therefore, the prevalence of a good picture in controller trainees (as opposed to naive subjects) was not directly tested. Experiments 1 focused on whether the accuracy of a student controller's picture is related to skill in air traffic control.

The picture assessment method used in the Preliminary Study was adapted for the TCTI radar simulation environment. The hypothesis of primary theoretical interest was to determine whether the accuracy of a student's picture is related to expertise in air traffic control. The support of this prediction would be given added weight if it also could be demonstrated that the ability to recall the picture is different from memory skill in general. In addition, testing to see if trainees recalled aircraft attributes in the same order as experienced controllers (Preliminary Study; Means et al., 1988; Bisseret, 1970) would give a general way of determining trainee skill level. Therefore, the specific predictions for Experiment 1 were:

1. ATC students' accuracy of recall of identifier, altitude, speed, heading, and position, and the rate of recall (the picture assessment) would account for a significant amount of variance in their scores on tests of air traffic control ability. 
2. Other measures of memory would not be correlated with the picture assessment and would not be associated with skill in air traffic control.

3. Students would show the same preference for certain aircraft attributes (altitude and position) as in previous studies.

Exploratory and applied themes included investigating the relationships between the criterion measures (academic grades, simulation achievement, and global ratings), determining the power of the DAT in predicting academic and practical achievement, and comparing trainees' memory test scores with normative data to assess levels of verbal and spatial memory. The usefulness of the variable set in discriminating between those who passed and failed the course was also of interest.

\subsection{Method}

\subsection{Subjects}

Two TCTI classes in the radar phase of training participated in Experiment 1. The first had 20 students with mean age and years education of 26.4 and 14.9, respectively, and the second had 17 students with mean age and education of 28.1 and 15.1, respectively. There were 19 males and 1 female in the first class and 13 males and 4 females in the second class.

The experimenter briefed potential subjects about the experiment and requested volunteers. The confidentiality of the results (in general and from their employer) was stressed. Volunteers were asked to sign a consent form and to provide name, age, home city, years of education, length of time at the school, and mailing address if a copy of the test results was desired. Each class was given a $\$ 100.00$ stipend when data collection was completed, but otherwise subjects were not paid over and above the salary they received from Transport Canada. All of the students in both classes agreed to participate. 


\subsubsection{Apparatus}

All subjects were given a battery of standard aptitude tests. This included the Verbal Reasoning, Numerical Ability, Abstract Reasoning, Clerical Speed and Accuracy, Mechanical Reasoning, and Space Relations subtests of the DAT. Each subtest required from 20.40 minutes to administer and was hand scored. Selected tests from the LAMB including List Learning, Complex Figure, and Working Memory were also given. (See Appendix B-3 for copies of the DAT and LAMB.)

The picture assessment procedure made use of the DEC10-based simulation system at TCTI. This simulator provided more realism than the computer program used in the Preliminary Study. Standard training exercises were available and the system had multiple controller workstations so that it was possible to run up to six subjects during each experimental session. Subjects had access to but were not directly monitoring the flight data strips.

The workstations were not physically modified. An 11 by 17 inch map (on a cardboard backing) was used for student responses (see Appendix B.1.3). The map was an enlarged photocopy of the Avalanche sector, 14 showing fixes, airways, and the sector boundary, but no labels. It was used at the school for testing trainee knowledge of the airspace. Although a blank map would have posed a more strenuous challenge to subjects' recall abilities and would have been more parallel with the hypothesized role of the controller's picture, ${ }^{15}$ it was decided to use a map which provided a basic geographical reference for the subjects. This would have the effect of focussing the recall task on the dynamic (as opposed to static) aspects of the picture.

14 This is a fictitious airspace created for training purposes.

15 If the controller's picture serves as a "supplementary display" as Whitfield (1979) suggested, a controller would use the picture to recall the attributes of a visually non-fixated aircraft while involved in some other task or while fixating on another area of the screen. The map background in the vicinity of the aircraft in question would not be visible. For this reason a page without map detail might be more appropriatc. 
Each map contained an area for subject information and an example of the format to be used to record aircraft data. Another set of maps was created for the simulation computer operators (or "pilots") for recording of actual positions of aircraft. Criterion measures of controller expertise included an average of the three final evaluation simulator tests (see Appendix B.3.1 for the form used), a global rating scale (adapted from Cobb, et al., 1973) completed by three instructors for each class (see Appendix B.3.2), and final academic grades.

\subsubsection{Procedure}

During the course of their radar training, subjects were given the DAT as a group in order to gather general information on verbal and non-verbal aptitudes. Testing was conducted in two or three sessions no more than one week apart. The memory tests were administered on a one-to-one basis. All testing was completed at least one week prior to the end of each class's term in radar training, but not necessarily before the picture assessments.

On the day of the picture assessment trials in the radar simulator, each class was assembled and given detailed instructions on the experimental procedure and response format. ${ }^{16}$ Subjects were informed that they would be involved in a standard simulation exercise and that it would be interrupted at an undisclosed time. The subjects were asked to turn at least 90 degrees away from their radar screens (the screens were also dimmed) and flight data strips and were instructed to record aircraft identifier, position, altitude, speed, and heading in a specific format.17 Subjects were told that they would be allowed as much time as required to record as many aircraft as possible. Both accuracy and speed were stressed. Practice was provided in how to record aircraft data.

16 See Appendix B.1.1 for the procedure.

17 Aircraft location was shown by a dot. Heading was indicated by a line extending from the dot in the direction of travel or a compass direction in degrees. Identifier was written below the dot. Altitude and speed were written below the identifier in the standard style (altitude in hundreds and speed in tens). 
The pilots were given identical maps and, at the point in time when the simulation exercise was stopped, were asked to carefully note the actual positions and other attributes of the aircraft from their displays. This allowed the experimenter to assess the accuracy of recall. In addition, the instructors (there was one for each student) were asked to time the subjects during each trial. 18

The two classes were each split into four groups for testing. Prior to each experimental run a map was given to each subject and simulator pilot and they were reminded of the procedure. After about 20 minutes (when there were eight aircraft active) the exercise was interrupted and subjects used their maps to record information about the aircraft they had been controlling. Simulator pilots recorded the actual positions of the aircraft. Each instructor kept track of the time required for each subject to recall as much information as possible After these tasks were completed, the exercise was restarted.

Initially it was planned to interrupt the simulation at two points in order to gather two sets of judgements from each subject. However, the procedure was altered after the first two groups in the first class had been run. The instructors were of the opinion that stopping twice was too disruptive of the training experience. The rest of the subjects in Experiment 1 were interrupted only at one point. Another modification was required due to a scheduling error. The last group in the first radar class was run on a different exercise, but one which was comparable to that used for the first three groups in this class. Al! subjects in the second radar class tested received consistent treatment.

The picture assessment procedure was completed during the third quarter (fourth or fifth week) of the class's radar training at TCTI. ATC Student Rating Forms fir each subject were gathered from three instructors for each class close to the end of the course. Final simulation and academic test results were collected after the completion of the course.

${ }^{18}$ Sec Appendix B.1.2 for the procedure. 


\subsection{Results}

Table 8 lists all of the variables employed in this study and their abbreviations. A number of summary measures were calculated in order to look for specific trends or to abbreviate the data set. Verbal reasoning and Numerical Ability were summed, as advised in the DAT manual. (VR+NA is supposed to be related to aptitude for academic work.) In the List Learning and Complex Figure memory tests the first two accuracy and time trials were summed and the differences between the last and first trials were calculated. Collaboration with the test author revealed that, based on experience with the measure, the use of mean performance for all trials was not advisable in that it does not capture improvements. The sum of the first two trials is suggestive of a subject's initial success with the test while the difference between the first and fourth trials shows the slope of the learning curve. On the third test the sum of errors of the more difficult trials (two and three back) were used as the measure of Working Memory.

As mentioned above, the two radar classes were each divided into four groups for testing. The first two groups of subjects in the first rada class were stopped twice during their experimental simulation runs. The data from the first stop time were used for these two groups because they represented the ATC trainees' initial experience with the task. All of the other groups of subjects were interrupted only once.

A problem with the scoring of position accuracy emerged when it was discovered that many subjects made large response errors with aircraft on the west side of the sector . Investigation revealed that the sector boundary on this side of the map was not in the correct place as compared to the radar display (see Appendix B.1.3). It was discovered that different westem and southern boundary lines are used depending upon whether the sector is "high" (above 18,000 ft.) or "low" (up to 18,000 ft.). Unfortunately, a low sector map was used with a simulation exercise of a high sector. Many subjects were disoriented by this mismatch and, when aircraft were close to the sector boundary on the 


\begin{tabular}{|c|c|}
\hline ABBREVIATION & DESCRIPTION \\
\hline AGE & age of subject \\
\hline EDUC & total years of education \\
\hline MCAT & score on ATC screening lest \\
\hline VR & DAT Verbal Reasoning \\
\hline NA & DAT Numerical Ability \\
\hline $\mathbf{V R}+\mathbf{N A}$ & $\operatorname{sum}$ of $V R$ and $N A$ \\
\hline AR & DAT Abstrect Reasoning \\
\hline CS\&A & DAT Clerical Speed and Accuracy \\
\hline $\mathbf{M R}$ & DAT Mechanical Reasoning \\
\hline SR & DAT Space Relations \\
\hline $\mathbf{F R 1 + F R 2}$ & sum of first two trials for List Leaming free recall \\
\hline FR5-FR1 & difference between last and first trial for List Leaming free recall \\
\hline CFI+CF2 & sum of first two trials for Complex Figure recall \\
\hline CF4-CFI & difference of last trial and first trial for Complex Figure recall \\
\hline CFT1+CFT2 & sum of response times for first two trials for Complex Figure recall \\
\hline CFT4-CFTI & $\begin{array}{l}\text { difference between last and first trial response times for Complex Figure } \\
\text { recall }\end{array}$ \\
\hline WKMTOT & sum of errors of two and three back trials for Working Memory \\
\hline NOAC & picture assessment: per cent of aincraft recalled \\
\hline IDENT & picture assessment: per cent of identifiers correctly recalled \\
\hline ALT & picture assessment per cent of altitudes correctly recalled (ballpark 19 ) \\
\hline$\overline{\mathbf{S P}}$ & picture assessment: per cent of speeds comectly recalled (ballpark) \\
\hline HDG & picture assessment: per cent of headings correctly recalled (ballpark) \\
\hline POS & picture assessment: per cent of locations correctly recalled (ballpark) \\
\hline RATE & pieture assessment: number of aincruft atributes recalied per minute \\
\hline ACAD & average of 6 academic tests in ATC training program \\
\hline SIM & average of 3 simulator final exam scores ( 4 in procedural phase) \\
\hline AVG RATE & average of 29 specific factors on global rating form (three instructors) \\
\hline AVGOALL & average of single overall ratings on global rating form (three instructors) \\
\hline AVG PICT & averase of picture ratings on global rating form (three instructors) 20 \\
\hline
\end{tabular}

Table 8. Variables used in the analysis of Experiment 1 and 2 data.

19 Sce Table A-3 for the criteria used to calculate balfpark accuracy for picture assessment.

20 AVG PICT is the average rating of the three instructors on the rating scale item (under "Comprehension") "Getling ane holding the picture". 
radar screen, tended to place them close to the sector boundary on the map. If the aircraft were close to the western boundary, this often resulted in a 30 mile error.

it was decided to omit position scores of aircraft which could possibly have been affected by this distortion. 21 In all, 23 subjects responded to simulation exercises in which some aircraft were in this situation and in these cases five of the eight targets were omitted from position scoring. The other subjects were stopped at different times or used a different exercise where none of the aircraft were close to the boundaries in question. However, this problem reduced the reliability of the average position error scores for the subjects who were affected.

Before combining the data sets for the first and second radar classes, t-tests of all of the relevant variables were conducted to look for systematic between-group differences. Table 9 shows the five tests which reached significance. Class two was superior to class one in their initial screening exam scores, the Clerical Speed and Accuracy subtest of the DAT, measures of altitude and speed error on the picture assessment, and the rate of recall of aireraft attributes (without counting accuracy) during the picture assessment. However, the classes were very similar in other predictor and outcome variables. There were no significant differences between male and female subjects. (Raw data are shown in Appendix C-1.1).

Upon further analysis of class one's results, the first two groups' poor performance on ALT, SP, and RATE accounted for much of the difference between the two classes on these variables. It may be that the subjects in the first two groups were more apprehensive about the procedure or that they knew they would be tested twice. Perhaps the number of anticipated interruptions interfered with their recall of the picture in some way.

21 However, none of the other aircraft attributes (identifier, altitude, speed, or heading) were problematic and were included in the arralyses. 


\begin{tabular}{|l|c|c|c|}
\hline VARIABLE & CLASS 1 MEAN & CLASS 2 MEAN & Yobs) \\
\hline MCAT & 42.41 & 44.75 & -3.04 \\
\hline CS\&A & 56.95 & 64.29 & -3.31 \\
\hline ALT & 64.76 & 82.98 & -2.51 \\
\hline SP & 31.99 & 76.37 & -4.33 \\
\hline RATE & 8.32 & 10.46 & -2.66 \\
\hline
\end{tabular}

Table9. Variables for which a significant difference existed between the first and second radar classes (p<.05). 
Although these between-class differences were found it was decided to combine the two data sets because the same procedures had been applied to both classes and the results appeared to be similar on most of the measures taken. A review of the variables was then commenced to look for univariate outliers and to test for normality of distributions (Tabachnick and Fidell, 1989).

With regand to the former, one subject was found to have scores on AR and RATE close to or more than three standard deviations from the mean (see Appendix C-1.2). Other subjects had an unusual scores on FR1+FR2, NOAC, POS, and ACAD. Scoring and data entry errors were ruled out and it was decided to retain the cases for further analysis but to replace these extreme scores by adding (or subtracting) one from the highest (or lowest) values in each variable (Tabachnick and Fidell, 1989).

When the distributions of the variables were reviewed (with the above changes in place), using tests of skewness and kurtosis recommended by Tabachnick and Fidell (1989), it was found that AR, NOAC, and POS were skewed (see Appendix C-1.3). However, multiple regression (the primary tool applied here) is robust with regard to using data containing deviations from normalcy (Pedhazur, 1982) and it was decided to refrain from transforming these variables, especially given that many of the measures showed some degree of negative skew. Tabachnick and Fidell (1989) suggested "if all the variables are skewed to about the same moderate extent, improvements of analysis with transformation are often marginal" (p. 84).

Pearson product-moment correlations were calculated between all of the variables in Table 8 to check for multicollinearity (see Appendix C-1.4). The only correlations over $r=$ 0.90 involved variables which were additive combinations of other variables or between variables measuring closely related factors from the same test. It was decided to employ regression diagnostics during the main analysis of the data to complete further screening for singularity, multivariate outliers, nonlinearity, and heteroscedasticity. 
While examining the correlation matrix, relationships between variables related to memory recall were noted. As can be seen in Table 10, the highest correlation was between ALT and FR5-FR1. It is noteworthy, given than the picture assessment procedure presumably relies on short term memory, that a stronger relationship was no: evident. To firther test this a canonical correlation was completed between the two sets of variables. No significant canonical variate emerged to relate the memory and picture assessment variables, although the number of cases was too small for this procedure to be very reliable (Tabachnick and Fidell, 1989).

When considering the relationships among the criterion measures (Table 11) a number $\mathrm{o}^{f}$ :trong correlations were present. Academic achievement in the radar course was not related to simulator test results or instructor ratings. However, simulation performance was strongly associated with instructor detailed and overall adequacy ratings as well as the assessment of how well the trainee could "get and hold the picture".

As in the Preliminary Siudy, an ANOVA was conpleted to determine if picture assessment accuracy varied according to aircraft attribute remembered. Although it was included in the analysis, the accuracy of recall of heading was thought to be artificially inflated. Almost all of the aircraft in each exercise were moving along familiar airways at the time the radar simulation was interrupted. Therefore, the likelihood of making a heading error was low as compared to the other artributes which had a much wider range of possible values.

The results of the ANOVA showed a main effect for accuracy of recall.22 Per cent correct for aircraft position was reported most precisely followed by heading, altitude, identifier, and speed. Scheffe tests were conducted between the remaining variables and significant differences were found between IDENT and HDG, IDENT and POS, SP,

22 See Appendix C-1.5.A for ANOVA data. 


\begin{tabular}{|l|c|c|c|c|c|c|c|}
\cline { 2 - 8 } \multicolumn{1}{c|}{} & FR1+FR2 & FR5-FR1 & CF1+CF2 & CF4-CF1 & CFT1+CFT2 & CFT4-CFT1 & WKMTOT. \\
\hline NOAC & 0.28 & -0.02 & 0.07 & -0.16 & 0.06 & 0.07 & 0.04 \\
\hline DENT. & 0.26 & -0.16 & 0.31 & -0.28 & -0.16 & -0.13 & -0.11 \\
\hline ALT & -0.29 & $0.48 *$ & 0.07 & -0.03 & -0.24 & 0.18 & -0.06 \\
\hline SP & 0.17 & 0.12 & 0.17 & -0.17 & -0.23 & 0.00 & 0.05 \\
\hline HDG & 0.18 & -0.08 & -0.03 & -0.09 & 0.03 & -0.04 & .0 .01 \\
\hline POS & -0.27 & 0.34 & 0.02 & 0.00 & -0.02 & -0.07 & .0 .19 \\
\hline RATE & -0.11 & -0.01 & 0.15 & -0.19 & -0.26 & 0.06 & 0.27 \\
\hline
\end{tabular}

Table 10. Pearson product-moment correlations between memory and picture assessment measures. (** significant at $\mathrm{p}<.01$, * significant at $p<.05)$.

\begin{tabular}{|l|c|c|}
\cline { 2 - 3 } \multicolumn{1}{c|}{} & ACAD & SIM. \\
\hline SIM. & -0.12 & \\
\hline AVG.RATE & -0.04 & $0.76^{*}$ \\
\hline AVG.OALL & 0.05 & $0.76^{*}$ \\
\hline AVG.PICT & 0.04 & $0.79^{*}$ \\
\hline
\end{tabular}

Table 11 . Correlations of criterion measures. ( ${ }^{+}$significant at $\left.p<.001\right)$. 
and HDG, SP and POS, SP and ALT.23 Figure 6 shows a graphical representation of the variables.

Multiple regression was applied to investigate the relationship of the predictor variables to the two criterion measures of interest (ACAD and SIM). Tables 12,13, and 14 present the results of these analyses. ${ }^{24}$

Table 12 shows the best multiple regression model for prediction of the average academic grade (based on five written tests given during the radar phase of training). Although the amount of variance accounted for only reached a modest $23 \%$, a test of the regression equation was significant as were tests of the regression coefficients for each variable ( $p<.05)$. According to this model, the possibility of academic success improves with the age of the trainee, the score on a test of working memory, and with the accuracy of recall of aircraft identifier from the picture assessment procedure. Inspection of the standardized regression coefficients indicates that the variables have about equal importance in the regression equation.

Table 13 shows a multiple regression with simulation performance as the dependent variable. The simulator score was an average of three final examination simulation runs and the marks were comprised of four factors: separation, coordination, and planning errors as well as an "affective rating" (see Appendix B.3.3 for a copy of the usstructor rating form).

The regression model accounted for a moderate amount of variance and the test of the regression was highly significant. Tests of all of the regression coefficients wers also significant ( $p<.05$ ). After inspection of residuals, leverages, ${ }^{25}$ and partial regression

23 Sce Appcndix C-1.5.B for Scheffé test data.

24 Regression diagnostics were used in each case to check for singularity, multivariate outliers, nonlinearity, and heteroscedasticity. Unless it is otherwise noted, no problems emerged.

25 "leverage" refers to how extreme a case is on its $x$-variables with regard to how much influence it exerts on the regression. 


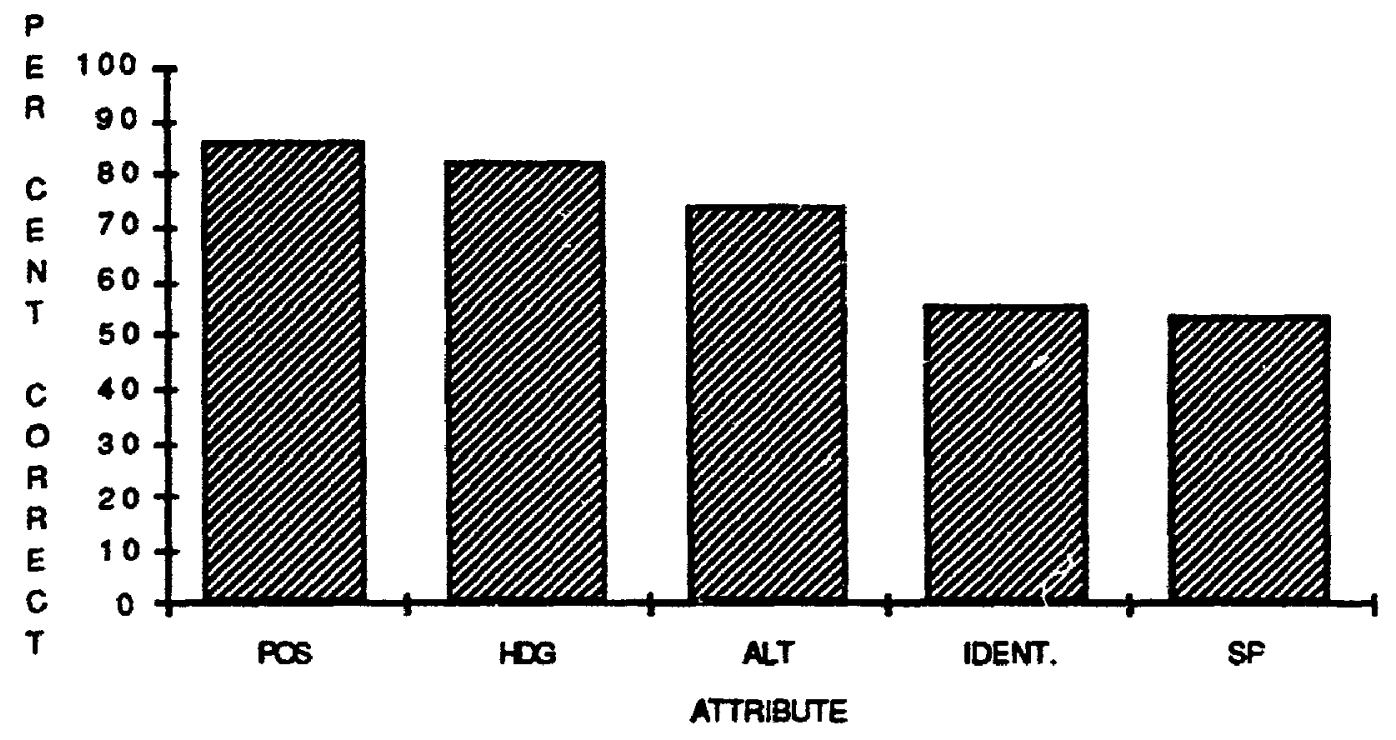

Figure 6. Mean per cent correct for aircraft atuributes recalled during picture assessment. 


\begin{tabular}{|c|c|c|c|c|c|c|}
\hline \multicolumn{2}{|l|}{ MULTIPLE R } & \multicolumn{5}{|c|}{0.55} \\
\hline \multicolumn{2}{|l|}{ MULTIPLE R ${ }^{2}$} & \multicolumn{5}{|c|}{0.30} \\
\hline \multicolumn{2}{|l|}{ ADJUSTED R ${ }^{2}$} & \multicolumn{5}{|c|}{0.23} \\
\hline \multicolumn{2}{|c|}{ SE OF ESTIMATE } & \multicolumn{5}{|c|}{3.61} \\
\hline \multicolumn{7}{|c|}{ ANALYSIS OF VARIANCE } \\
\hline & \multicolumn{2}{|c|}{$\mathbf{S S}$} & df & MS & \multicolumn{2}{|l|}{ ERATIO } \\
\hline REGRESSION & \multicolumn{2}{|c|}{179.07} & 3 & 59.69 & \multicolumn{2}{|l|}{4.58} \\
\hline RESIDUAL & \multicolumn{2}{|c|}{417.24} & 32 & 13.04 & \multicolumn{2}{|l|}{$p<.01$} \\
\hline YARIABLE & $\mathbf{b}$ & & SE & B & 1 & D(2TAIL) \\
\hline$A G E$ & 0.32 & & 0.13 & 0.37 & 2.46 & 0.02 \\
\hline WKMTOT & 0.35 & & 0.14 & 0.36 & 2.43 & 0.02 \\
\hline IDENT & 0.05 & & 0.02 & 0.32 & 2.13 & 0.04 \\
\hline CONSTANT & 66.71 & & & & & \\
\hline
\end{tabular}

Table 12. Regression of average academic test score over selected predictor variables. 


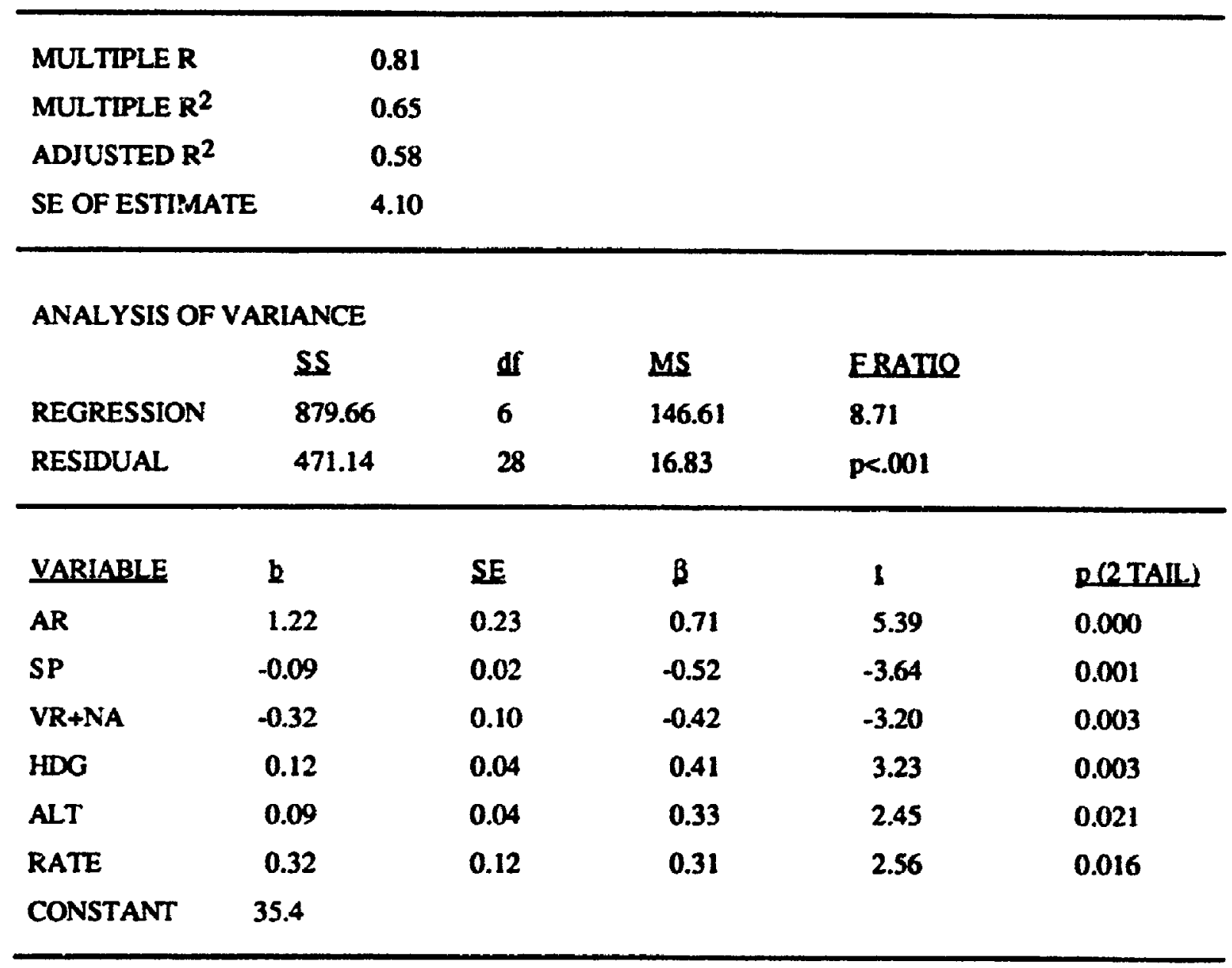

Table 13. Average simulation performance (three trials) regressed over selected predictor variables. 
plots, ${ }^{26}$ one case was noted as a multivariate outlier. Having obtained a low simulation grade, this subject nevertheless obtained average or above average scores on five of six of the predictor variables. This pattern of results did not fit the regression model and the case was removed from the analysis.

As was mentioned earlier, the first two groups of subjects to undergo the picture assessment were stopped twice during the simulation. These analyses employed the first set of judgements for the first two groups. As a check, another regression model using the second set of data for the first two groups was created. It was composed of the same set of predictor variables with the exception of RATE.2? This result indicates that the regression model for the prediction of simulation score is robust.

The variables in the equation are listed in order of importance (as indicated by standardized regression coefficients and generally confirmed by $t$ values). AR had the largest positive correlation. However, SP and VR+NA were negatively correlated with level of practical skill. HDG, ALT, and RATE were positively related to simulator performance.

In order to consider the unique importance of the trainees' picture in predicting simulation performance only the picture assessment variables were entered into a regression equation. Table 14 shows the results. In addition to the subject noted as a multivariate outlier in the previous analysis two further cases were removed. One subject obtained average or high scores on all of the predictor variables in Table 14 but had a very poor simulator grade. Another subject, mentioned earlier as a univariate outlier with a very rapid RATE, created a large amount of leverage in the regression.

26 A partial regression plot "graphs $y$ with the linear effects of the other $x$-variables removed against $x$ with the linear effects of the other $x$-variables removed" (Velleman \& Velleman, 1988, p. 23.4).

27 It should be noted that this data set did not undergo the checks for outliers applied to the primary data set. Such procedures may have improved the performance of the RATE variable. 


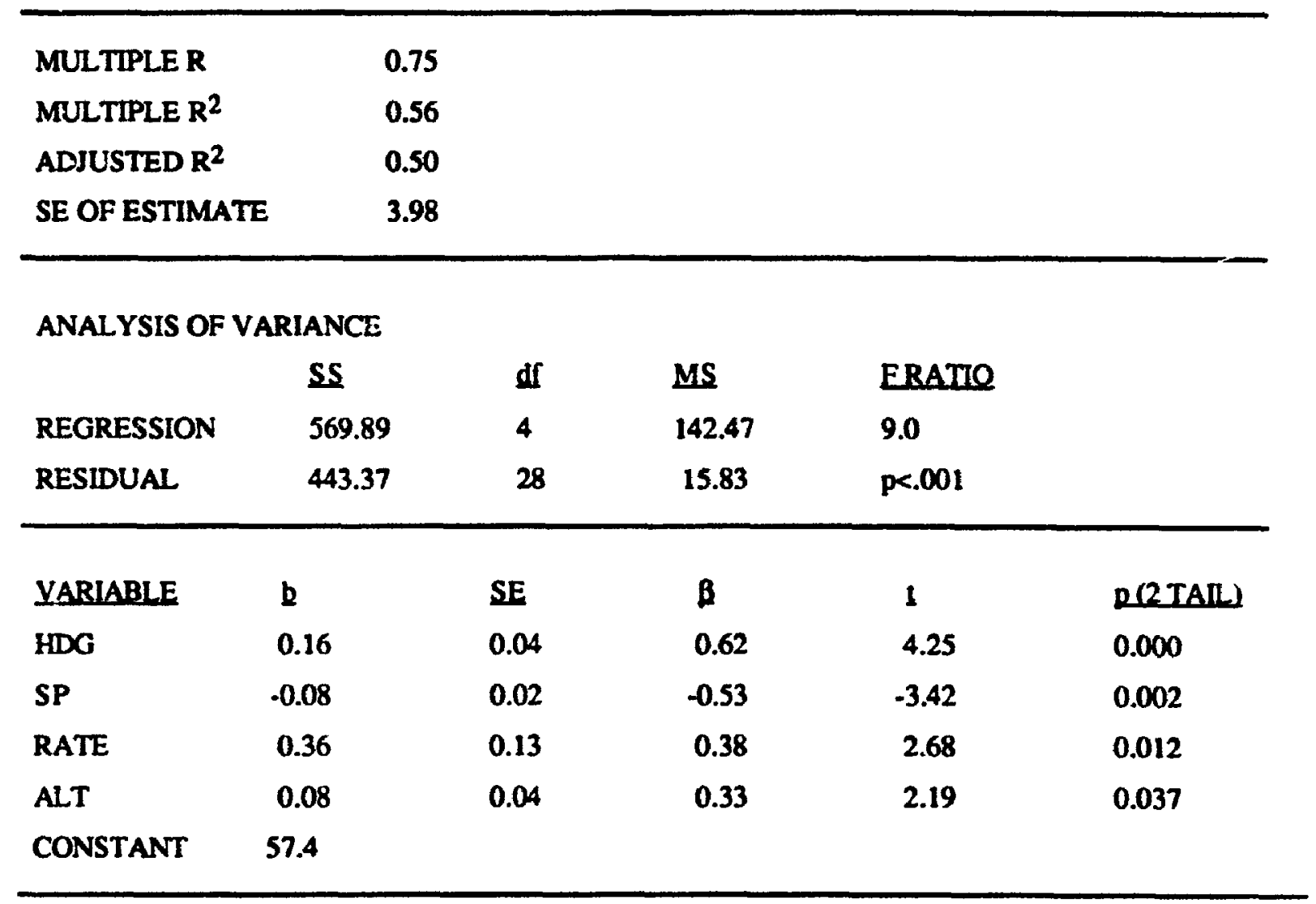

Table 14. Average simulation performance (three trials) regressed over picture assessment variables. 
Table 14 indicates that the variables in the model accounted for at. It $50 \%$ of the variance in simulation performance and that the regression was significant. This table also displays the variables in order of importance (based on standardized regression coefficients). HDG was the most important factor followed by SP, RATE, and ALT. SP was again negatively correlated with practical radar ability. Tests of the coefficients were significant $(p<.05)$.

A stepwice discriminant function analysis was conspleted to determine whether any subset of the variables could reliably distinguish between students who passed or failed the radar training program. In this analysis, all cases were left in and Table 15 presents the results. As can be seen, the discriminant function was $90 \%$ correct in classifying subjects who passed the course and $100 \%$ correct in categorizing those who failed. The variables accounted for $45 \%$ of the variance in the data set. AGE and VR+NA were negatively correlated with success in the program while EDUC, CFT4-CFT1, and SR were positive indicators. Give the small number of subjects available, this analysis can only be considered as exploratory.

\subsection{Discussion}

It is interesting that only one of the measures of memory in Table 10 (FR5-FR1) correlated significantly with any of the picture assessment variables (ALT and POS). FR5-FRI relates to list learning efficiency and may relate to the requirement for leaming labels and numbers in order to remember the picture. However, given that this is presumably not related to being able to remember position (which is spatially coded), it may be that rate of learning is the common factor.

It is revealing that none of the other memory test and picture assessment variables were correlated to any degree. The memory recall skills involved in the picture assessment probably rely on the ability to apply knowledge to remember many aircraft attributes (or the ability to chunk). Training creates knowledge which supports the perception of data on the 


\begin{tabular}{lllll}
\hline CANONICAL CORRELATION & 0.67 & & & \\
WILKES LAMBDA & 0.55 & & \\
CHI SQUARE & & 18.43 & DEGREES OF FREEDOM & S $\quad$ P .003 \\
\hline YARIABLE & WILKES'LAMBDA & SIGNIECANCE \\
AGE & 0.90 & 0.060 \\
EDUC & 0.80 & 0.029 \\
CFT4-CFT1 & 0.63 & 0.002 \\
VR+NA & 0.74 & 0.029 \\
SR & 0.71 & 0.025 \\
\hline
\end{tabular}

\section{CLASSIFICATION MATRIX}

$\begin{array}{lll}\text { GROUP } & \text { PASS } & \text { EAIL } \\ \text { PASS } & 26 & 4 \\ \text { (CORRECT) } & (86.7 \%) & (13.3 \%) \\ \text { FAIL } & 0 & 6 \\ \text { (CORRECT) } & (0.0 \%) & (100.0 \%)\end{array}$

PER CENT OF CASES CORRECTLY CLASSIFIED $\mathbf{8 8 . 9 \%}$

\begin{tabular}{lll}
\hline VARIABLE & STANDARDIZED CANONICAL & CORRELATIONS OF \\
\cline { 2 - 3 } & DISCRIMINANT FUNCTION & $\frac{1}{\text { VARIABLESAND CANONICAL }}$ \\
COEFFICIENTS & DISCRIMINANT EUNCTIONS \\
EDUC & 1.00 & -0.35 \\
CFT4-CFT1 & 0.86 & 0.31 \\
VR+NA & -0.64 & -0.24 \\
SR & 0.56 & 0.18 \\
AGE & -0.34 & -0.37 \\
\hline
\end{tabular}

Table 15. Stepwise discriminant function analysis for the pred iction of pass/fail outcome in the radar training class. 
radar display as being meaningful. While some subjects showed an ability to use chunking skills to remember the picture this did not correspond with the ability to recall unrelated words, an abstract figure, or with working memory.

Although it might be argued that chunking skills are also helpful for the retention of LAMB material, a basis for making the stimuli meaningful has to be invented in such tests and does not rely on applying a body of knowledge in which the stimuli are embedded. This suggests that those who do well on the LAMB may not be those who remember the picture accurately. These results support the second hypothesis stated earlier and imply that maintenance of the picture involves a speciai kind of memory ability.

When the results of memory testing were averaged over the entire group, radar class performance could be checked against norms for the List Learning and Complex Figure acquisition trials (Tombaugh \& Schmidt, 1989). ${ }^{28}$ The cumulative percentile scores were: List Learning free recall $39^{\text {th }}$ pctl., List Learning free plus cued recall $42^{\text {nd }}$ pctl., and Complex Figure $87^{\text {th }}$ petl. These results indicate that the students had below average abilities for remembering a list of unrelated words but had superior skills when a complex figure was to be recalled.

The correlations reported in Table 11 showed that instructor ratings of trainee ability were highly correlated with simulator performance. This is not surprising given the practical orientation of the rating scale (see Appendix B.3.4). However, the low correlations of overall academic grades with simulator and instructor evaluations indicate that classroom performance is not necessarily related to ATC skill level. The theoretical training given to students may not adequately address important practical issues. Altemately, good performance on tests of knowledge may not necessarily imply that a trainee will be able to apply this leaming in simulated operational conditions.

28 No norms were available for the Working Memory test. 
It was again found that accuracy of recall of radar screen information favoured position and altitude as opposed to identifier and speed. However, the data regarding position must be interpreted with a degree of caution due to the limited number of observations involved. This result supports hypothesis three and suggests that the trainees prioritized aircraft attributes in the same way as experienced controllers. This could be taken as a rough indication that they possessed similar knowledge structures.

The critical sections of the data analyses, for the purpose of establishing the importance of the picture, are the multiple regression models. Table 12 demonstrates the best prediction equation for average academic performance. In general, it appears that the aptitude, memory, and picture assessment variables measured during this study are not very successful in accounting for proficiency in the classroom. This result is puzzling given that subtests from the DAT are widely used to assess aptitude for academic achievement. Increasing age, perhaps due to more maturity and motivation to succeed, is a factor. The other two variables, Working Memory and recall of aircraft identifier, are interesting in that they both involve verbal memory in different ways. This is not surprising in that the academic tests require that a large amount of written material be memorized. There is only one item from the picture assessment included in the regression model. However, the quality of the picture was not be expected to be related to academic success.

This is not the case for the regression model predicting simulator performance. As shown in Table 13, four of the picture assessment variables were included as significant predictors of sirnulation competence thus supporting the first hypothesis. Reviewing the predictors in order of importance, Abstract Reasoning (a non-verbal measure of general reasoning ability) had the highest positive correlation with practical skill. An abstract reasoning measure also proved to be useful in an FAA selection test battery (Sells \& 
Pickrel, 1984). The next two variables, speed and the sum of Verbal Reasoning and Numerical Ability, were both negatively correlated with simulation score.

The indication that high scores on these factors are counterindications of ATC skill is counterintuitive. With regard to the recall of aircraft speed from the radar display, instructors' comments on its importance are helpful. In general, they emphasize that attention to speed is unwise. When separating aircraft, changes to altitude and heading are recommended as the most effective interventions. Speed is nc: a factor because large differences in the velocities of two aircraft must exist to have any effect on separation over short time periods. Sperandio (1978) observed in his research on workload in air traffic control that speed was a variable which was poorly represented by zontrollers as workload increased. It is in these comments that we may find the explanation for the negative correlation of speed recall with simulator performance. Students who stressed this factor may have a good picture but have emphasized one of the wrong attributes, perhaps to the exclusion of other critical information.

Verbal reasoning and numerical ability could be useful skills in air traffic control. However, when considered separately or together, they were negatively correlated with simulator outcomes. This is in contrast with the strong positive effect a good score in nonVerbal Reasoning had on the criterion measure. When these three variables are considered together a strong statement is made about the importance of non-verbal as opposed to verbal skills. It also is possible that the verbal abilities tapped by the DAT are not those which are critical for air traffic contrcl.

The remaining predictors included heading and altitude accuracy as well as the rate at which subjects completed the recall task. This suggests that knowledge of the direction of travel and altitude of targets on the radar display as well as the spoed with which the picture can be reconstructed are important skills for air traffic control. 
It is again interesting to consider which variables were excluded from the regression model. Clerical speed and accuracy, Mechanical Reasoning, ard Space Relations did not emerge as important factors. The DAT literature notes that Space Relations measures the ability to "manipulate things mentally, to create a structure in one's mind from a plan.... It is an ability needed in such fields as drafting, dress designing, architecture, art, diemaking, and decorating -- wherever there is a need to visualize objects in three dimensions" (Bennett, et al., 1988, p. 9). Perhaps the ability to create a three-dimensional image from the radar screen is not important in radar control.

None of the memory tests identified any critical dimensions of controller skill (supporting the second hypothesis). As mentioned above, it is possible that the tests selected for this research do not tap the ability to employ knowledge to chunk effectively; this may be the critical memory factor for retention of the picture. It also indicates that good general short term recall abilities (as tested by the LAMB) may not be critical for ATC.

The number of aircraft recalled, identifier, and position also did not have much weight in the equation. It has been noted previously that identifier is not stressed as an important attribute in recall studies. Much of the position information was contaminated by measurement problems. If this had not been the case, this factor would have been expected to play a role in the trainee's picture, as suggested by the good recall of position in the Preliminary Study.

Age, years of education, and MCAT score were also not included. Although there is no specific reason to expect any influence of the first two factors, the MCAT is used to select controllers and it is surprising that it does not play a role. However, the test is used to screen applicants. All those who participated in this study had already surpassed the required cutoff score. It may be that the MCAT is not good at making fine discriminations regarding performance once a student is in school. 
Table 14 concentrates on the picture assessment variables and here accuracy of recall of heading emerged as the most important factor. Knowing the direction in which aircraft are travelling would be critical for maintaining separation. Speed was again negatively cortelated while RATE and altitude had a smaller but significant influence. This result demonstrates that the accuracy of the trainees' picture by itself contributes substantially to the level of practical expertise.

All three of the hypotheses stated carlier were supported in the above results. The subjects, although they were trainees, appear to have a similar knowledge base to experienced controllers. The regression results provided an convincing demonstration that the ability to maintain an accurate picture of certain aspects of the radar display is imporant for skilful air traffic control. Recall of the picture appears to involve a different kind of chunking skill to what might be employed in standard memory tests.

Inspection of Table 15 indicates that a stepwise discriminant function analysis using an initial set of nine variables was $90 \%$ successful in categorizing the students who passed and $100 \%$ correct with those who failed. According to the standardized coefficients of the variables, the order of importance was education, the time difference (over four trials) to recall and draw a complex figure, the sum of Verbal Reasoning and Numerical Ability, Space Relations, and age. As before, VR+NA was negatively correlated with passing the course. However, in contrast to its relationship to average academic grade, increasing age was not a predictor of success.

The discriminant analysis created a set of predictor variables which had only two factors in common with the multiple regression analyses. Age was useful in predicting academic score and Numerical Ability emerged as a factor in simulator performance. Education, CFT4-CFT1, and Space Relations were part of the discriminant function although they had played no role in the regression models. Most notable was the absence of any of the picture assessment variables. The correlations of the variables with the 
canonical discriminant function suggests that the underlying factor for success in training is related to education and aptitude as opposed to memory or picture-related skills. ${ }^{29}$

The lack of agreement between the regression and discriminant analysis variables is probably due to the use of different criterion measures. The multiple regression models had only average academic or simulation score as the dependent variable. The discriminant function predicted a more compiex event, membership into one of two groups: pass or fail. This was decided by a more complex procedure than average final exam score.

Students had to achieve $80 \%$ in every academic exam to be allowed to take the simulator tests. In each exercise a student had to achieve a minimum score on instructor ratings of separation, coordination, planning, and affective rating. ${ }^{30}$ Assuming this requirement was met, the sum of the scores on these four variables then made up the result for a simulation run and two of three of the exercises had to be scored over $70 \%$ in order for the student to pass. Although four of the six failing students achieved an average over the three simulations of $70 \%$ or more, they made errors which cause them to fail two of the exercises. Five students obtained an average score in the same range as those who failed but were nevertheless placed in the "passed" group. The critical variables which determined success in these cases were planning and separation.

The scoring system used to determine success or failure in the course weighted separation, coordination, planning, and affective rating unequally; this is presumably based on the school's philosophy of what is important for safe and efficient air traffic control.

29 However, it is difficult to interpret the meaning of scores on CFT4-CFT1. An improvement in response time over the four acquisition trials for the complex figure (resulting in a negative score) might indicate an increasing rate of task completion. On the other hand, a positive score could merely reflect the inclusion of more detail in the later drawings. Inspection of subject scores showed both positive and negative results. Correlations of CFT4-CFT1 with CF4, CF1+CF2, and CF4-CF1 were nonsignificant indicating that CFT4-CFT1 is not related to amount of detail remembered.

30 The "Affective Rating" is composed of scores on separation visualization, planning priorities, board management and stripwriting, phraseology and communications, coordination and teamwork, and confidence and comportment. 
There was also evidence that instructor discretion was employed with borderline cases. On the other hand the criterion used in the regression equations involved an equal weighting of all four factors. Only further research will demonstrate which approach for evaluating skill in air traffic control is the best predictor for success in on-the-job training and for eventual licensing. 


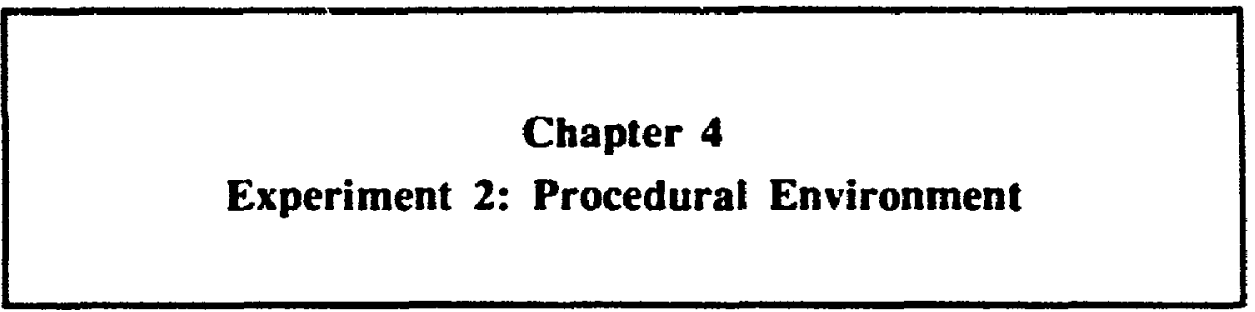




\subsection{Introduction}

In Experiment 1 it was found that the accuracy of a student's picture of aircraft in a radar simulation was related to measures of skill. It would also be useful to show that the accuracy of the picture is an important factor in the procedural air traffic control environment. The number of subjects involved in Experiment 2 was not as large as in Experiment 1. Nevertheless, Experiment 2 provided data which in part confirms the findings from Experiment 1 and suggests topics for further research.

Experiment 2 was very similar to Experiment 1 . However, in this case the picture assessment occurred as part of a procedural simulation exercise. The same aptitude and memory test batteries and criterion measures were employed. As in Experiment 1 the hypothesis of primary theoretical interest was to determine whether the accuracy of the picture was related to expertise in air traffic control and whether the ability to recall the picture was different from short term memory skill in general. In addition, comments from previous studies (Means et al., 1988) suggested that different skills would be involved in procedural work. Lastly, priority of recall by the students was again compared with previous studies.

The specific predictions for Experiment 2 were:

1. ATC students' accuracy of recall of identifier, altitude, speed, heading, and position, and the rate of recall (the picture assessment) would account for a significant among of variance in their scores on tests of air traffic control ability.

2. Other measures of memory would not be correlated with the picture assessment and would not be associated with skill in air traffic control.

3. The factors important for procedural control would be different from those in radar control with an emphasis on variables associated with spatial imagery (such as Space Relations, Complex Figure, POS, and ALT). 
4. Students would show the same preference for certain aircraft atributes (altitude and position) as experienced controllers.

Issues for exploration and of practical interest included looking into the relationships . between the criterion measures (academic grades, simulation achievement, and global ratings), determining the power of the DAT in predicting academic and practical achievement, and comparing trainees' memory test scores with normative data to assess levels of verbal and spatial memory.

\subsection{Method}

\subsubsection{Subjects}

As in Experiment 1, subjects were volunteers who were attending TCTI in the procedural control phase of air traffic control training. There were a total of 17 students (16 males and 1 female) in the class tested (average age 25.9 years, average education 15.0 years). It had originally been expected that 24 students would be available but seven withdrew from the training program for various reasons including illness and due to problems passing the academic tests.

\subsubsection{Apparatus}

At TCTI procedural simulation was conducted in a special room divided into two areas, one for the simulator pilots and the other for those practicing their air traffic control skills. Each section had twelve workstations consisting of a flight data strip rack (or "strip bay"), a digital clock, and an intercom. Students took turns being pilots or controllers. Information about an aircraft was printed on a series of flight data strips, one for each navigational marker the aircraft would fly over. Given the aircraft's route and the time it was supposed to reach each fix, controllers could keep track of its progress through the airspace. Flight plans were updated by voice contact with pilots and any changes in aircraft altitude, speed, etc. were noted on the flight data strips. 
This simulation system was used for Experiment 2. As in Experiment 1, two preexisting (but equivalent) exercises were employed in order to interfere as little as possible with the ongoing training program. It was possible to run four or five subjects during each experimental run. All other apparatus was identical with Experiment 1 except that a different airspace map was used (see Appendix B.2.3).

\subsubsection{Procedure}

The procedure for Experiment 2 was identical with Experiment 1, except that the instructor assigned to each student was asked to record the actual position, identifier, altitude, speed, and heading of each aircraft (instead of a computer system operator). The procedural simulator was not computer-based and the instructors consulted the students' flight data strips for this information. Another modification was that the data was collected at the end of the simulation run rather than during an interruption as in Experiment 1. However, it should be emphasized that seven or eight aircraft were still active at that time so that the traffic load was comparable to Experiment 1. (See Appendices B-2.1 and B-2.2 for the procedures used.)

\subsection{Results}

The data set ${ }^{31}$ was reviewed for univariate outliers and only three scores were found to deviate more than three standard deviations from the mean.32 One subject had unusual values on SR and ALT while another showed an outlier on CS\&A. It was decided to retain the cases for further analysis but to replace these extreme scores by adding (or subtracting) one from the highest (or lowest) values in the variable. With the outliers modified in this way the data were screened for skewness and kurtosis. ${ }^{33}$ No unusual distributions were discovered, as indicated by tests recommended by Tabachnick and Fidell (1989).

31 See Appendix C-2.1 for data.

32 Sec Appendix C-2.2 for data

33 Sce Appendix C-2.3 for data. 
Pearson product-moment correlations were calculated between all variables to check for multicollinearity and singularity. ${ }^{34}$ The only correlations over $r=0.90$ were between some of the outcome criteria which were undoubtedly measuring overlapping information. It was decided to employ regression diagnostics during the main analysis of the data to complete further data screening for singularity, multivariate outliers, nonlinearity, and heteroscedasticity. ${ }^{35}$

Table 16 focuses on the section of the correlation matrix for the picture assessment and memory test variables. There were some correlations of $r=0.40$ or more but, as in Experiment 1, there was not much of a relationship between the two sets of variables. In this case a canonical correlation was not completed due to the small number of cases.

Table 17 shows the correlations among the criterion measures. In contrast to the results in Experiment 1, academic achievement had a moderate (but non-significant) positive correlation with simulator test results and a significant correlation with instructor ratings. As before, simulation performance was strongly related to instructor detailed and overall adequacy ratings as well as to the assessment of how well the trainee could "get and hold the picture".

An ANOVA was completed to determine if picture assessment accuracy in the procedural environment varied as a function of aircraft attribute remembered. The results showed a main effect for accuracy of recall of aircraft attributes. ${ }^{36}$ Per cent correct for aircraft heading was reported most precisely followed by altitude, position, speed, and identifier. Scheffe tests were conducted and significant differences were found between IDENT and HDG, IDENT and POS, IDENT and ALT, SP and HDG, SP and ALT, and HDG and POS. ${ }^{37}$ Figure 7 shows a graphical representation of the variables.

\footnotetext{
34 See Appendix C-2.4 for data.

35 Unless it is otherwise noted, no problems emerged.

36 See Appendix C-2.5.A for ANOVA data

37 See Appendix C-2.5.B for Scheffé test data.
} 


\begin{tabular}{|l|c|c|c|c|c|c|c|}
\cline { 2 - 8 } \multicolumn{1}{c|}{} & FR1+FR2 & FRS-FR1 & CF1+CF2 & CF4-CF1 & CFT1+CFT2 & CFT4-CFT1 & WKMTOT \\
\hline NOAC & 0.16 & -0.12 & -0.03 & 0.42 & 0.19 & 0.07 & 0.06 \\
\hline IDENT & $0.49 *$ & -0.27 & 0.30 & -0.07 & -0.03 & 0.19 & -0.05 \\
\hline ALT & -0.02 & 0.02 & 0.10 & -0.12 & -0.15 & 0.04 & -0.28 \\
\hline SP & 0.45 & -0.19 & 0.26 & -0.04 & 0.01 & 0.28 & 0.02 \\
\hline HDG & 0.03 & -0.09 & -0.09 & 0.29 & 0.24 & -0.04 & -0.09 \\
\hline POS & -0.05 & 0.10 & 0.20 & -0.05 & 0.44 & -0.07 & 0.39 \\
\hline RATE & -0.32 & -0.16 & -0.18 & 0.08 & -0.16 & -0.27 & 0.10 \\
\hline
\end{tabular}

Table 16. Pearson product-moment correlations between memory and picture assessment measures. (* significant at pe .05).

\begin{tabular}{|l|c|c|}
\cline { 2 - 3 } \multicolumn{1}{c|}{} & ACAD. & SIM. \\
\hline SIM. & 0.37 & \\
\hline AVG.RATE & $0.67^{* * *}$ & $0.81^{+}$ \\
\hline AVG.OAL & $0.63^{* *}$ & $0.84^{+}$ \\
\hline AVG.PICT & $0.59^{*}$ & $0.88^{+}$ \\
\hline
\end{tabular}

Table 17. Correlations of criterion measures.

(* significant at p<.05, ** significant at $p<.01$, *** significant at $p<.005$, + significant at $p<.001)$. 


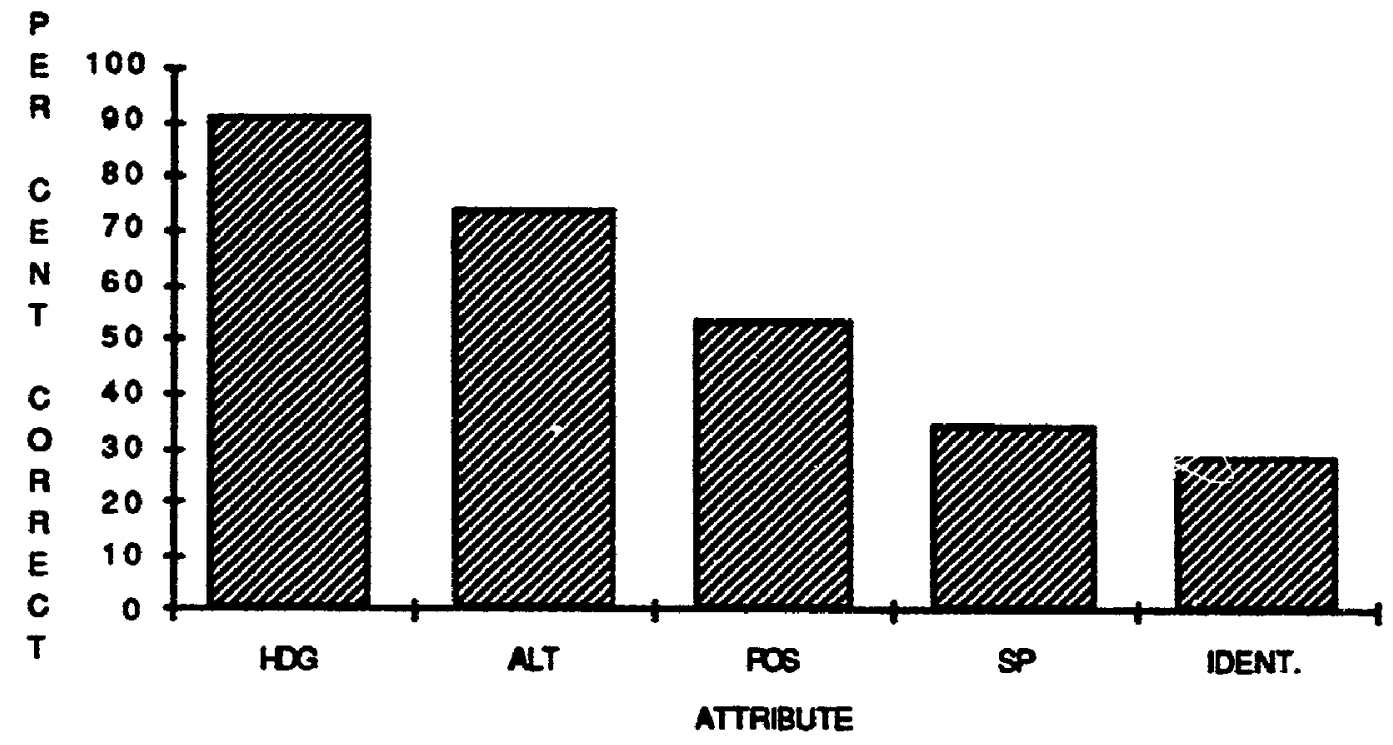

Figure 7. Mean per cent correct for aircraft attributes recalled during picture assessment. 
As in the first experiment multiple regression was employed to investigate the relationship of the predictor variables to the two criterion measures of interest (ACAD and SIM). Tables 18 and 19 present the results of these analyses.

Table 18 shows the best multiple regression model for predicting the average academic grade (based on five written tests given during the procedural phase of training). The amount of variance accounted for was $57 \%$ and a test of the regression equation was significant as were tests of the regression coefficients for each variable (p<.05). In the case of academic grades, the likelihood of success improved (in order of importance) with the the rate at which a trainee completed the picture assessment task: the time difference between first and fourth trials in recalling the complex figure, and the number of aircraft included in the picture assessment. (One subject was left out of the equation due to a missing value for RATE.)

Table 19 lists the results of a multiple regression analysis with simulation performance as the dependent variable. The simulator score was an average of four final examination simulation runs and the marks are comprised of four factors: separation, coordination, and planning errors as well as an "affective rating". (Appendix B.3.3 shows a copy of the IFR Training Report.)

The regression model accounted for $66 \%$ of the variance in the simulation grades and the test of the regression was significant. Tests of all of the regression coefficients were also significant $(p<.05)$. After examining residuals, leverages, and partial regression plots, one case was identified as a multivariate outlier. This subject was not able to complete most of the picture assessment task. He had a poor simulation run and was upset at the time he was asked to complete the map. The subject's pattern of results did not fit those of the other students and his case was removed. 


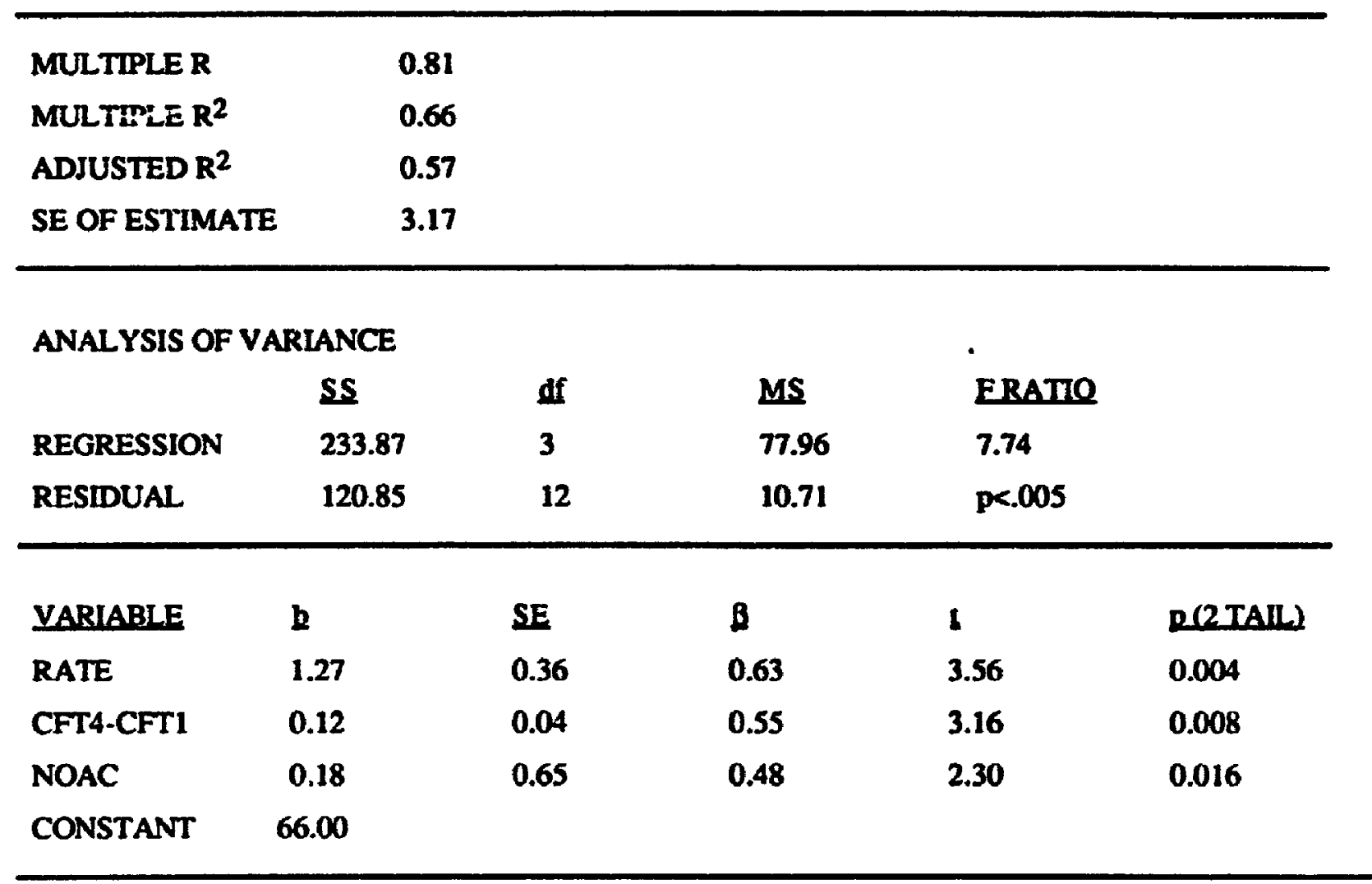

Table 18. Average academic performance regressed over selected predictor variables. 


\begin{tabular}{|c|c|c|c|c|c|c|}
\hline MULTIPLER & \multicolumn{3}{|c|}{0.87} & & & \\
\hline \multicolumn{2}{|l|}{ MULTIPLE R ${ }^{2}$} & \multicolumn{2}{|c|}{0.75} & & & \\
\hline \multicolumn{2}{|l|}{ ADJUSTED R 2} & \multicolumn{2}{|c|}{0.66} & & & \\
\hline \multicolumn{2}{|c|}{ SE OF ESTIMATE } & \multicolumn{2}{|c|}{3.80} & & & \\
\hline \multicolumn{7}{|c|}{ ANALYSIS OF VARIANCE } \\
\hline & \multicolumn{2}{|c|}{$\mathbf{S S}$} & df & MS & ERATIO & \multirow[b]{3}{*}{ - } \\
\hline REGRESSION & \multicolumn{2}{|c|}{476.08} & 4 & 119.02 & 8.26 & \\
\hline RESIDUAL & \multicolumn{2}{|c|}{158.53} & 11 & 14.41 & $p<.005$ & \\
\hline VARIABLE & $\mathbf{b}$ & & $\underline{\mathbf{S E}}$ & B & 1 & p(2TAI) \\
\hline HDG & 0.30 & & 0.10 & 0.45 & 2.29 & 0.014 \\
\hline WKMTOT & -0.95 & & 0.38 & -0.40 & -2.51 & 0.029 \\
\hline AR & -0.09 & & 0.36 & -0.38 & -2.43 & 0.033 \\
\hline AlT & -0.16 & & 0.07 & -0.37 & -2.33 & 0.040 \\
\hline CONSTANT & 133.91 & & & & & \\
\hline
\end{tabular}

Table 19. Average simulation performance (three trials) regressed over selected predictor variables. 
HDG was the most important factor in the equation (as indicated by standardized regression coefficients and generally confirmed by $t$ values). Then followed WKMTOT, $A R$, and ALT. The latter three variables were negatively correlated with the dependent variable. In contrast, $A R$ and ALT were positively correlated with simulator performance as they were in the radar class results.

The variables measured in the picture assessment procedure were then entered into a regression equation. However, only one of these factors proved to be significantly related to practical skill level. HDG correlates $r=0.54$ with $\operatorname{SIM}, F(1,14)=5.78, p<.05$. A stepwise discriminant function analysis was not completed with this data set due to the small number of subjects.

\subsection{Discussion}

In the procedural environment, a different measure of list learning (FR1+FR2) was related to some of the picture assessment variables (IDENT and SP), as shown in Table 16. In addition, there were marginal correlations of the Complex Figure recall task (CF4-CF1 and CFT1+CFT2) with number of aircraft recalled arid position. There is a suggestion here of relationships between variables which tap verbal mernory (FR1+FR2, IDENT, and SP) versus spatial memory (CF4-CF1 and NOAC, CFT1+CFT2 and POS). However, as in the previous experiment, the lack of many significant correlations suggests that the memory abilities measured by the two procedures focus on different kinds of mnemonic abilities ard lends supports to the second hypothesis.

When the results of memory testing are averaged for the procedural class, performance can be checked against norms for the List Learning and Complex Figure acquisition trials (Tombaugh \& Schmidt, 1989). ${ }^{38}$ The cumulative percentile scores were: List Leaming free recall $55^{\text {th }}$ pctl., List Learning free plus cued recall $65^{\text {th }}$ peti., and

38 No norms were available for the working memory test. 
However, at TCTI altitude and speed are suppressed so that the only thing the trainee saw in the radar data block was the aircraft identifier. Therefore, it may be important to keep altitude in mind in the radar environment whereas this may not be useful in non-radar control.

The fact that WKMTOT, the ability to keep inisrmation in memory for later use while simultaneously entering new information, was also negatively weighted in the regression model suggests that retaining items in memory may interfere with the cognitive activities required in procedural control. It may be important to shed rather than retain certain information and to be able to shift tasks quickly. The availability of all necessary aircraft information on the flight data strips and the intense mental operations involved in predicting future position without visual work aids may favour devoting mental capacity to computation rather than retention. Further study is required to better define the meaning of this and the other implications suggested in the procedural simulation regression model.

Most of the DAT subtests (including Space Relations) do not play a role in this regression equation. A number of the picture assessment variables were also not useful predictors of non-radar skills. The recall of speed and identifier were not critical and, although no problems with scoring position accuracy were experienced in the procedural study, position was also not a factor.

The low importance of speed and identifier suggested by the recall accuracy results (see Figure 7) may help explain this. However, it is curious that altitude and position were remembered quite accurately but were not positively related to simulator skills. The fact that procedural control involves working with time ${ }^{42}$ (rather than geographical location of aircraft) may partially explain this. Irrespective of the overall averages for altitude and

42 Each aircraft is expected to pass over specific radio beacons at certain times and the simulation is run forward based upon a clock readout. Therefore, much of the work involves the prediction of time to cross over the next fix based upon speed and distance. There was no component in the picture assessment which addressed the recall of time predictions. 

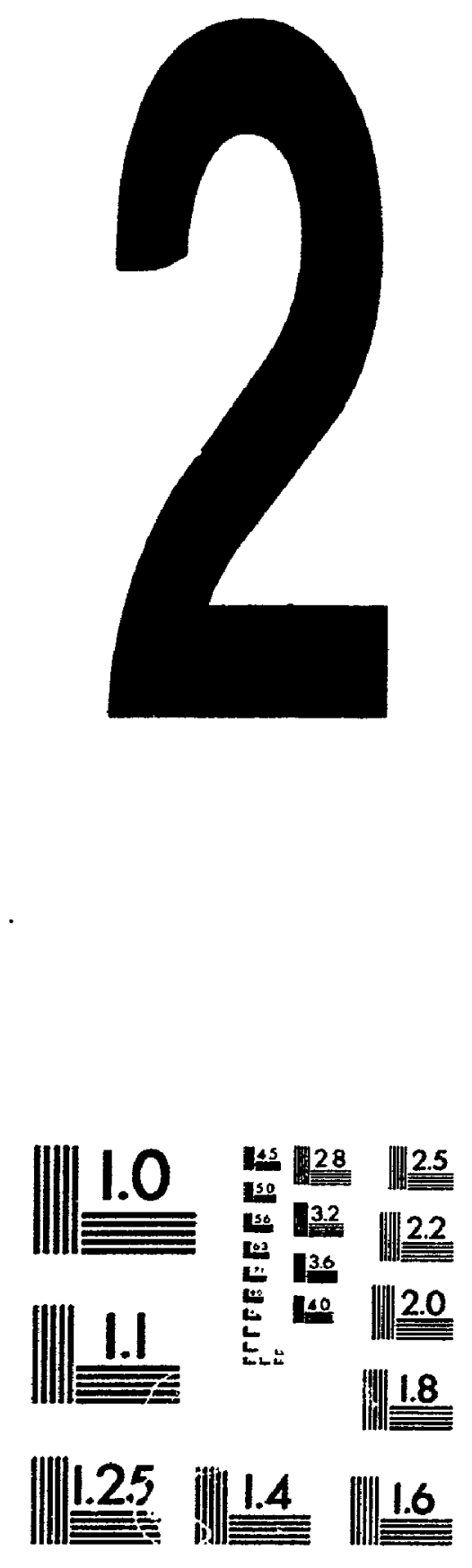

MICROCOPY RESOLUTION TEST CHART

NATIONAL BUREAU OF STANDARDS

STANDARD REFERENCE MATERIAL 1010a

(ANSI and ISO TEST CHART NO. 2) 
Complex Figure $82^{\text {nd }}$ pctl. The students had above average abilities for remembering a list of unrelated words (better than the radar classes) and had superior skills for recalling a complex figure.

Table 17, correlations of criterion measures, indicates a different pattern from the radar class. Although procedural simulator scores were again highly related to instructor ratings, academic results showed a much stronger correlation with these judgements than before. This may indicate that instructors in the procedural training segment are considering both academics and practical skills when making their assessments. However, given that the ATC Student Rating Form is oriented toward skills as opposed to knowledge, it is more probable that the classroom work in the procedural phase is more directly related to the task of controlling aircraft than in the radar phase.

With regard to the accuracy of recall of aircraft attributes (Figure 7), the same general pattern of prioritizing altitude and position as in the radar classes and previous research was followed (supporting the fourth hypothesis). However, compared to the radar group the procedural students were significantly less accurate in recalling position but more accurate with heading and identifier (there were $n\lrcorner$ significant differences in recall of altitude or speed). 39 However, their order of priority was different. Altitude and position still took precedence but altitude rather than position was recalled more accurately. (The accuracy of response for heading would again be expected to be inflated because all aircraft were travelling either east or west.) Speed and identifier were also reversed in ordinal relationship.

The regression model which best predicted academic success (Table 18) accounted for more variance than in the radar classes (57\% as opposed to $23 \%$ ). However, the fact that the radar statistics were based on an inhomogeneous blend of two classes probably

${ }^{39}$ Sce Appendix C-3. for t-test data. 
reduced the predictive power of the regression model so it would not be wise to place too much weight on this difference. However, it is interesting that RATE, CFT4-CFT1, and NOAC are now in the model as opposed to AGE, WKMTOT, and IDENT. This may reflect the difference in subject matter between the two courses.

Academic success in the procedural environment depended upon the number of aircraft recalled (without regard to accuracy) in the picture assessment task, how quickly this was accomplished, and the difference in rate of completion of Complex Figure recall between first and fourth trials. In the procedural situation, it appears that some aspects of the ability to form a picture are related to academic success. It may be that the basic training for forming the picture is provided at this phase. This is supported by the fact that two of the academic topics are "Navigation and Airspace" and "Aircraft". The other factor, CFT4CFT1, has an uncertain interpretation, as discussed in Experiment 1.

The DAT measures and most of the memory tests again did not emerge as good predictors of academic achievement. In other situations where it is important to predict . academic success, the DAT has been shown to be useful. These results indicate that a standard aptitude battery like the DAT may not be ideal for the selection of ATC trainees.

When predicting the outcome of simulator work there were differences in the variables included in the model (as compared to Experiment 1). AR, ALT, and HDG were still present. However, AR and ALT were negatively correlated with outcome. WKMTOT was the only other variable in the model. It did not appear in the radar results and it had a negative weight in the procedural equation. 40 The first hypothesis is partially supported by these resilts in that two of the six picture assessment variables appeared in the equation.

It is interesting that Abstract Reasoning (a ineasure of non-verbal problem solving ability) was negatively correlated with success in the procedural phase of training while it

40 Here only four variables were included in the model in an attempt to observe the 5:1 cases-to-variables ratio advised for multiple regression by Tabachanic and Fidell (1989). 
was a strong positive predictor in the radar environment. There were indications in the radar setting that verbal and numerical skills were not useful. However, Verbal Reasoning was helpful for procedural control (VR was borderline significant).41

An explanation for the reversal in importance of non-verbal and verbal aptitudes may lie in the contrasting demands of the procedural and radar environments. In the latter, the controller was presented with a detailed pictorial display of the air traffic situation whereas in procedural control there were only flight data strips arranged in a rough geographical configuration. The regression iesults suggest that non-verbal problem solving may be the best approach for radar and that good verbal abilities may be counterproductive. On the other hand, a particular strength in abstract reasoning appears to be a hindrance in the procedural environment. The borderline significance of VR has theoretical importance because it suggests that non-radar work may require verbal mediation. Students who earned good scores in both abstract and verbal reasoning were not those who did well in either simulation environment. These results are more understandable if it is assumed that aptitude test scores reflect not only certain abilities but a cognitive style a subject brings to bear on many kinds of problems.

Another curious finding was the negative correlation of the recall of aircraft altitude with simulator outcome. This was the reverse of its relationship to success in radar simulation. If it can be assumed that there is limited memory capacity for the recall of picture elements, it would be counterproductive to remember unnecessary details. The fact that aircraft altitude is constantly available and updated on the flight data strips in procedural control may mean that it is not important to keep it in mind in favour of other aircraft attributes. In the radar environment, altitudes are normally displayed on the screen.

41 Verbal Reasoning emerged as a predictor of simulation performance in the regression equation but a ttest of its slope was only significant at $p<.10$. It was not included in the regrestion model because of the limit imposed by the variables to subjects ratio requirement and because a cutoff of $p<.05$ had been chosen for slopes. 
However, at TCTI altitude and speed are suppressed so that the only thing the trainee saw in the radar data block was the aircraft identifier. Therefore, it may be important to keep altitude in mind in the radar environment whereas this may not be useful in non-radar control.

The fact that WKMTOT, the ability to keep inisrmation in memory for later use while simultaneously entering new information, was also negatively weighted in the regression model suggests that retaining items in memory may interfere with the cognitive activities required in procedural control. It may be important to shed rather than retain certain information and to be able to shift tasks quickly. The availability of all necessary aircraft information on the flight data strips and the intense mental operations involved in predicting future position without visual work aids may favour devoting mental capacity to computation rather than retention. Further study is required to better define the meaning of this and the other implications suggested in the procedural simulation regression model.

Most of the DAT subtests (including Space Relations) do not play a role in this regression equation. A number of the picture assessment variables were also not useful predictors of non-radar skills. The recall of speed and identifier were not critical and, although no problems with scoring position accuracy were experienced in the procedural study, position was also not a factor.

The low importance of speed and identifier suggested by the recall accuracy results (see Figure 7) may help explain this. However, it is curious that altitude and position were remembered quite accurately but were not positively related to simulator skills. The fact that procedural control involves working with time ${ }^{42}$ (rather than geographical location of aircraft) may partially explain this. Irrespective of the overall averages for altitude and

42 Each aircraft is expected to pass over specific radio beacons at certain times and the simulation is run forward based upon a clock readout. Therefore, much of the work involves the prediction of time to cross over the next fix based upon speed and distance. There was no component in the picture assessment which addressed the recall of time predictions. 
position in Figure 7, the regression results showed that subjects who recalled this information well were not those who obtained high scores in the simulator.

The regression data partially supported the fourth prediction listed in the introduction that a different set of skills and picture elements would be required for procedural control. As discussed above, there was a reversal in the correlations of non-verbal and verbal reasoning skills and WKMTOT was present. Recall of heading also continued to play a role but RATE and SP were omitted and ALT carried a negative slope. However, there was no indication of the importance of spatial imagery as might be incicated by the presence of significant positive coefficients for SR, CF1+CF2, POS, or ALT.

Given that the three of the hypotheses from Section 4.1 were supported, there is an indication (as in Experiment 1) that the trainee's picture is also important in procedural control. However, the evidence is weaker in that only two of the picture assessment variables were present in Table 19 and one was negatively correlated with skill. Contrary to the conventional wisdom about procedural air traffic control, there was no evidence that spatial imagery was important. 


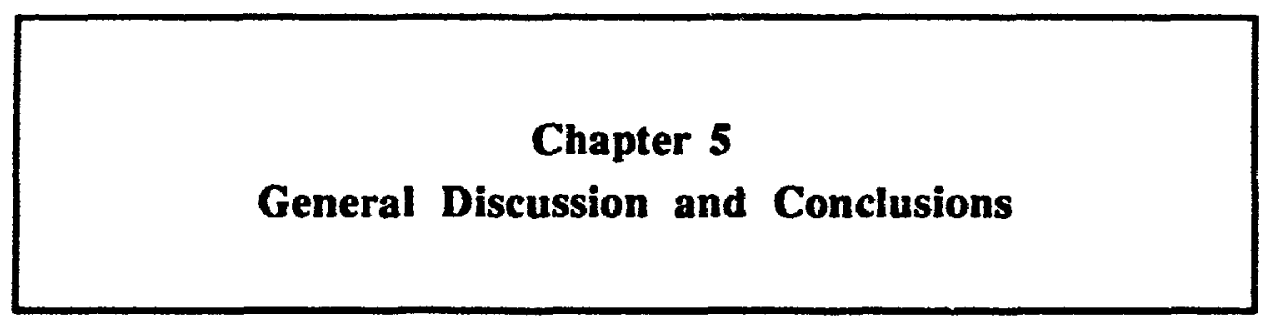




\subsection{Overview}

In this chapter the results of the Preliminary Study and Experiments 1 and 2 are reviewed with respect to theoretical and practical issues. The theoretical findings evolved from specific hypothesis testing and from exploration of the data. However, in the previous chapters it has been difficult to separate out results which had applied ramifications. These will be emphasized at a later point in this chapter along with suggestions for further research. Finally, concluding remarks will point to critical findings about the air traffic controller's mental model.

\subsection{Theoretical Issues}

With the goals of exploring the air traffic controller's mental model in terms of its descriptivis, explanatory, and predictive power in mind, the Preliminary Study was designed to demonstrate that experienced air traffic controllers had certain specific abilities. The set of hypotheses stated in Section A-1.4 were intended to address the structure, organization, resolution, spatial dimensionality, and resiliency of the controller's picture and to test the controller's ability to map and predict in three-dimensional space using a two-dimensional stimulus (the radar screen). It was assumed that differences between controllers and non-controllers would highlight the characteristics of the mental model resulting from training and experience.

Appendix A and Chapter 2 review the findings of the Preliminary Study. Although clearer results might have obtained with more subjects and a smaller set of experiments, useful information nonetheless emerged. As has already been discussed, experienced controllers showed superior and generally more rapid recall abilities for airctift information. They preferred to remember altitude and position over other aircraft data.

Controllers turned their heads less often to view the display while working on a plotting task. On average they checked the screen about every nine seconds while students looked every six seconds. Moray (1979) reported research that showed air traffic 
controllers lose $15 \%$ of aircraft status information in 15 seconds and that memory for a simulated radar display begins to fade rapidly after about 12 seconds. Given that subjects were probably self-prompted to refresh their image of the radar screen as it began to lose clarity, the times observed in the Preliminary Study fall within the range of these data.

The theme of the hypotheses supported in the Preliminary Study is that controllers are distinguished from other subjects in their ability to retain data about aircraft. The good results of one or two of the non-controllers suggest that this skill may be partially aptitudebased and applied even over the short time period of the Preliminary Study experiments. 43 However, controllers showed more consistent performance. Differences in the way controllers visualize and organize information and in their spatial and predictive abilities did not emerge (except in one experiment).

Other useful theoretical results emerged from the Preliminary Study in addition to those generated from hypothesis testing. There was evidence in the recall and cluster analysis data that the picture subjects developed during the experiments was structured in certain ways, presumably by a complex underlying knowledge base. The work of Landis, et al. (1967) discussed earlier suggested that skilled controllers have qualitatively different knowledge structures than those with less expertise. This was also echoed by Rouse and Morris (1986). Vincente (1988) reviewed the literature on differences in problem solving between experts and trainees and said "...the knowledge representation of novices is organized according to surface characteristics, but that of experts is based upon functional principles" (p. 19). Chase and Ericsson (1982) came to the same conclusions when they

43 Some of the non-controllers in the Preliminary Study were probably able to build up a workable mental model of the ATC system. TRACON is advenised as a simulation or game which does not require lengthy training in ATC to use. The knowledge naive subjects accumulated about TRACON from the training and practice included in the experiments may explain how some of them performed as well as the air traffic controllers on some tests. The more experienced subjects, on the other hand, were probably not called upon to employ much of their knowledge about ATC. 
found that an extensive knowledge base was important for improving memory span for cigits.

A specific expression of the organizational forces at work in the controller's mental model is found in the correlation of order of recall of aircraft with their perceived priority of importance in the traffic scenario. The subjects in both the Preliminary Study and Experiments 1 and 2 also consistently preferred to recall certain kinds of aincraft data (altitude and posicion). Sperandio (1978) noted (with regard to ATC) that "Recall is organised hierarchically, according to the frequency with which information is used in reasoning" (p. 198).

During the Preliminary Study controllers judged aircraft similarity using binary rather than continuous criteria. This was also observed by Bisseret (1981) who suggested that it is important for controllers to make a rough categorization of aircraft as a basis for quick action. It may be that this behaviour is indicative of an "impulsive" as opposed to a "reflective" mental strategy. "Reflectivity refers to a tendency to search systematically for the best solution given a choice of alternative responses. Impulsivity refers to making rapid responses at the expense of careful, slower selections" (Anshel \& Ortiz, 1986, p. 1311). Although the term "impulsive" may connote acting carelessly without thinking, Bisseret (1981) observed that the controllers in his study were quite safety conscious.

The amount of aircraft information a controller can remember is impressive. In the Preliminary Study, experienced controllers were able to accurately recall about $48 \%$ of the 45 aircraft attributes on the screen (or 22 separate pieces of information). ${ }^{44}$ Clearly, the controller's picture can contain considerable detail. It is probable that the ability to chunk a large amount of information is a primary requirement for good performance air traffic control.

44 Some subjects recalled up to 35 elements correctly (ballpark accuracy). 
An adaptation of Pinker's (1980) methodology for studying mental imagery indicated that some subjects visualized the radar screen in three dimensions. This suggests that there may not be a unified approach to the perception of radar displays. Pethaps users vary in their preferences and abilities for three-dimensional space visualization when confronted with a plan view of aircraft with numerical coding of altitude. The use of imagery may also depend upon task demands. When the third dimension is visualized, it appears that subjects expand their spatial image of altitude (when viewing a map of airspace). This finding may prove to be a generalization of Thomdyke's (1980) work on the filled distance effect (as discussed in Appendix A).

The final theoretical point raised by the Preliminary Study is that subjects who were able to detect errors during simulations were also those who accurately recalled aircraft data (see Appendix A). If it can be assumed that the recall task is a measure of the picture, these data suggest that the quality of the mental model is related to effective fault detection. Human factors research on automation provides support for this statement. Wickens (1980) reported experiments which showed that operators were less accurate and slower in detecting system malfunctions when they were monitoring ("out of the loop") rather than in direct control. It has been suggested that the monitoring role probably results in an incomplete mental model of a system. Those in the Preliminary Study who could not remember the picture had similar problems with fault detection.

The primary theoretical result emerging from Experiments 1 and 2 is that the controller's picture, as measured by the picture assessment procedure, is an important factor for good performance in a simulated air traffic control environment. In Experiment 1 the accurate recall of aircraft heading and altitude and the rate with which the picture was reconstitured were significant predictors of success in the simulator. This finding is reinforced by studies conducted by the FAA into the characteristics of the air traffic controller. One of their conclusions was "The central skill of the controller seems to be the 
ability to respond to a variety of quantitative inputs about several aincraft simultaneously and to form a continuously changing mental picture to be used as the basis for planning and controlling the courses of aircraft" (Dailey, 1984).

A review of the dynamics of memory will assist in the interpretation of the data from Experiments 1 and 2. Hitch (1987) distinguished between the temporary demands which computer-based tasks place on short term or working memory as opposed to the more lasting requirements involving long term memory (episodic and semantic). These distinctions are reminiscent of Whitfield's (1979) statement that the controller's picture relies on working memory and a dynamic model (short term retention) as well as a static model (long term memory).

Temporary demands on memory during work with a computer include the need to retain temporary labels and parameters, the user's current subgoal, the user's current response, and the present state of the computer (Hitch, 1987). The present research on the controller's picture probably addresses only the first of the above categories. Working memory is the cognitive mechanism which supports the retention and retrieval of this information. ${ }^{45}$ It is composed of a "general purpose 'central executive' linked to specialpurpose stores, a 'visuo-spatial scratchpad' and a speech-based 'articulatory loop'. The central executive is an attentional system responsible for a wide range of control processes which include accessing and manipulating the contents of the special-purpose stores" (Hitch, 1987, p. 123).

The fact that the LAMB tests did not predict trainee skill suggests that good working memory by itself is not the essential factor. Trainees' memory test results ranged from

45 "Working memory" is Hitch's (1987) term for shor term storage and has, a different meaning from the kind of recall assessed by the Working Memory test used in this research. The LAMB test focuses on a specific aspect of working memory which is often called "running memory". For the discussions in this chapter only, "working memory" will be synonomous with short lerm memory and "running memory" will refer to the LAMB test. 
below average on verbal tests to above average for spatial material. However, the regression models showed that the aptitude for chunking a large amount of relevant aircraftrelated information is the critical factor. This probably involves the application of knowledge to create a meaningful and memorable set of information from the data displayed on the screen or flight data strips.

The negative regression coefficient for LAMB Working Memory (or running memory) in the procedural environment suggests that students who are able to hold simple information in memory for subsequent use while simultaneously entering new information do not perform well in simulated ATC tasks. As discussed in Chapter 4, this result could be explained if those subjects who have an aptitude for running memory habitually retain old information even when new information is presented. Moray (1979) described research which showed that running memory for "the two-back condition imposed a very great load on the operator when he was required to perform a tracking task at the same time" (p. 32). This could certainly apply to air traffic control. A predisposition to let aircraft data "stick" in memory in favour of new information may limit the amount of attention a traince can allocate to controlling aircraft in the here-and-now. It also may be that the measure of running memory in the LAMB which focuses on digits addresses the wrong content area. Hitch (1987) observed that results of a working memory test in one domain may not relate to performance in another domain.

There was further evidence in Experiments 1 and 2 that particular kinds of information are not practically important or may even create interference if held in the picture (speed was an example in the radar setting as was altitude in procedural control). Therefore, retention of a good picture of the air traffic control situation may not simply include memorizing all the information available but, given the restrictions of short term memory, selecting which elements are important and ignoring others. 
This interpretation is supported by studies on the capacity limitations of short term memory. Research has shown that when subjects are asked carry on a complex task while concurrently performing a separate, short-term storage task (such as digit span), decrements in primary task performance increase as the memory load approaches span (Hitch, 1987). A finding more relevant to air traffic control is that "Similar interactions between concurrent storage load and processing speed have been reported in complex tasks where the temporary storage load is intrinsic to the task rather than extrinsic" (Hitch, 1987, p. 129). Therefore, it would be counterproductive to retain any more information than is required while involved in a task as demanding as air traffic control. It may be that the more accomplished students not only remembered well but had leamed to manage the contents of their memories effectively (or had a good central executive).

Although there was evidence that the accuracy of the ATC trainee's picture is related to radar simulation performance, there was not as good an indication of this in the procedural environment. In Experiment 1 four of the variables from the picture assessment predicted simulator outcome with a reasonable degree of accuracy. However, only two out of the set of picture variables correlated to any significant degree with procedural expertise. This result does not support the conventional wisdom in ATC that procedural control requires a higher degree of visualization (and a better picture) than when radar is available (Means, et al., 1988). It is possible that the picture assessment procedure did not tap the kind of mental imagery used in procedural control.

It is evident from a comparison of the results from Experimenis 1 and 2 that the picture which is important for radar control is different from that required in procedural control. The fact that altitude was an element of the picture in the radar simulator but not in the procedural environment relates back to Whitfield's (1979) earlier statement that the mental model may act as a supplementary display. One reason altitude is mentally represented when using radar may be that it is not shown on the screen but is nevertheless 
important for controlling aircraft. This situation would force the subject to check the flight data strips or communicate with the aircraft in order to obtain altitudes and to hold these data in memory. 46 However, in procedural operations, all of the air traffic information is constantly updated by the student on flight data strips. Thus, there may be no need to represent altitude in the picture given that it can be obtained through visual search as needed. Perhaps this ready availability of aircraft data allows the controller in the procedural environment to generate a picture which is at a higher level of abstraction (Johnson-Laird, 1983; Rasmussen, 1979).

The prediction of academic success in the two experiments was not a primary focus of interest. Nevertheless, it is worth noting that all of the variables (except for age) in the regression equations were related to the recall of various kinds of information. None of the DAT subtests emerged as being important despite the fact that it was designed to measure aptitudes for various occupations. These results emphasize the role of memory in learning the large number of procedures and facts necessary for ATC.

The DAT results in Experiments 1 and 2 turned out to be a rich source of information. It was puzzling that Space Relations did not play any role in the regression equations (although it emerged in the discriminant function for Experiment 1). There are two possible reasons for this. One is that the criterion measure used to assess practical skill (instructor ratings of simulation performance) did not address abilities dependent upon Space Relations. This interpretation is suggested by inspection of the correlations of DAT tests with various criterion measures in work by the FAA on ATC trainee selection (Brokaw, 1984). In both enroute and terminal FAA academy trainee samples, Space Relations correlated significantly with some outcome measures but not with others. In both

${ }^{46}$ Although flight data strips are present in the radar control setting, another controller manages and updates them. The radar controller's primary display is the radar screen and he or she must tum away from this to look at the flight data strips. 
groups this factor had low correlations with supervisor rating and (in the terminal sample) with laboratory score. The importance of Space Relations for predicting ATC skill was more dependent on the criterion measure selected as compared to other DAT variables. It is also interesting to note that Brokaw (1984) reported poor results in using DAT Mechanical Reasoning as a predictor of ATC ability, as was also found in the present study.

The other explanation for the poor performance of Space Relations may be due to issue of construct validity. The ability measured by the DAT may not be that which is important for air traffic control. Cooper and Mumaw (1985) reported the results of a major reanalysis of studies on spatial aptitude which consistently identified three subfactors of this aptitude. "The first, Spatial Relations, represents the ability to solve simple mental rotation items quickly. The second subfactor, Spatial Orientation, represents the ability to imagine how a stimulus array will appear after a reorientation of the body.... The third subfactor... is Visualization" (Cooper \& Mumaw, 1985, p. 69). It may be that the DAT Space Relations test measures an aspect of spatial aptitude (presumably the first subfactor) which is not as relevant to air traffic control as some other factor, such as Visualization. These authors also noted that Space Relations generally correlates with visual memory. If Visualization is the critical dimension, this may explain the lack of importance of LAMB Complex Figure recall in the regression models despite generally good student scores on this test.

The pattem of verbal and non-verbal DAT test scores in the two experiments suggests that these skills may be either relevant or disadvantageous, depending upon the simulation environment. This is suggested by the reversal in sign of the regression weights of $A R$ and VR (+NA) between Experiments 1 and 2.47 Abstract reasoning appears to be helpful in the

47 As mentioned in Experiment 2, NA was a marginally significant variable which was not shown in Table 19. The level of significance of its slope $(p<.10)$ resulted in its exclusion from the procedural simuation regression model. It nonetheless has meaning in the interpretation of the verbal/non-verbal results in the two experiments. 
radar environment but numerical ability and verbal reasoning skills may impede performance. In the procedural simulator, verbal reasoning is an asset while abstract reasoning is a hindrance.

Caplan (1984) and Cooper and Mumaw (1985) have suggested that a high level of skill in a certain aptitude could induce a preference for solving problems in that mode. The pattern of results for verbal and non-verbal abilities in Experiments 1 and 2 suggests that subjects who approach radar or procedural simulation with a specific cognitive style will be more successful than those who have a more unbiased ("bicognitive") approach or who apply the opposite style.

The term "cognitive style" describes an individual's habitual way of processing information (Caplan, 1984). A number of cognitive style dimensions have been identified and explored. One of the more well known continuums is field dependence-field independence (Witkin, Moore, Goodenough, \& Cox, 1977). Others include holisticanalytic (Cooper \& Mumaw, 1985), abstract-concrete (O'Conner, 1972), and hemispheric (Caplan, 1984). This last dimension is described in the following:

According to this perspective, in those instances in which a problem may be efficiently solved by more than one method, certain individuals will exhibit a hemispherically-based cognitive strategic performance. For instance, some subjects might attempt to remember a list of words by rote repetition (lefthemispheric, verbal analytic strategy), while others might seek to create a mental picture containing images of the to-be-remembered words (righthemispheric, visual holistic strategy). (Caplan, 1984, p. 280)

It appears from the pattern of DAT results that a visual/holistic style works best with radar control and a verbal/analytic approach is most effective in the procedural setting. Students who had the opposite pattern of aptitudes in a given environment (and who presumably used a cognitive strategy commensurate with their abilities) performed poorly as did those with no pronounced style.

Other research reported by Cooper and Mumaw (1985) indicates that for some subjects cognitive strategy may be completely determined by aptitude level. However, for 
others aptitude does not affect the initial strategy choice but determines the efficiency in using the chosen strategy (with abler individuals performing better). An important component of general aptitude may be strategy flexibility, but:

...holistic subjects can adopt the analytic mode when task-demands naturally draw upon that strategy. However, only under unusual conditions can analytic subjects adopt the holistic mode. Furthermore, when analytic subjects are induced to switch strategies, they use the holistic mode less efficiently than do the 'natural' holistic individuals. Thus, it appears that holistic processors are more flexible and efficient than analytic processors. (p. 83)

One reason students who graduate from the procedural phase of training fail when confronted with radar may be that they are analytic processors and have difficulty making the shift to the visual/holistic mode. Such considerations may have led Dailey (1984) of the FAA to comment (regarding the skills necessary for air traffic control) "The combination of these mental demands requires that the controller be a 'perceptual-discrimination' athlete. This means that the job of the air traffic controller is beyond the capability of the average person and therefore a high degree of selection is required for the occupation" (p. 134).

Statements of controllers (Whitfield, 1979), other researchers (Brokaw, 1984), and the results of Experiments 1 and 2 support the hypothesis that air traffic controller's picture (especially in the radar environment) is not an epiphenomena but is an important cognitive tool for controlling aircraft. Although the research reported here was completed with students using a simulator, there is reason to believe that these findings would also apply in an operational setting with licensed controllers. This generalization is based upon the assumption that the dependent measure (simulator performance) is a realistic assessment of air traf: tc control skill. There is a good likelihood of this because it is relied upon as a primary method of evaluating trainee expertise at the school. Boone (1984) supported the use of "ATC laboratory average score" in his FAA studies on selection testing by observing that "...prior studies had demonstrated that these scores were the most reliable predictors of subsequent success as an Air Traffic Controller" (p. 377). However, only further 
experiments with licensed controllers will completely demonstrate the importance of the picture.

\subsection{Practical Applications}

The Preliminary Study identified some tools which could be effective in exploring the controller's picture. It is evident that the recall of screen information can indicate what appears to be critical in the controller's mind. Cluster analysis holds promise for showing how the data are organized. In addition, controllers tend to have a dichotomizing decisionmaking strategy (or impulsive-reflective style) and assessment of this personality trait may prove to be useful in candidate selection.

The most potentially powerful result from this research comes from Experiment 1 (and to a lesser extent Experiment 2) where it was demonstrated that the picture (as measured by the picture assessment procedure) is related to practical skill (in the radar simulator). These results may be of considerable importance for selection testing given that radar is the dominant working environment in ATC. It might be possible to identify applicants who possess the ability to apply their ATC knowledge to form an accurate picture.

Nearly all of the variables relating to academic success in both the radar and procedural phases of training involved memory. This suggests that the ability to leam and remember large amounts of information could be a critical factor for success in this part of ATC training. Some way of assessing memory could be considered for screening applicants, although it would be necessary to find out more about the specific mnemonic skills required. The finding that increasing age may be counterindicative of good grades should also be considered. However, caution should be observed in using standard aptitude test batteries like the DAT for predicting academic performance.

The discriminant analysis in Chapter 3 suggested that a good chance of predicting success in the second phase of training could be achieved using some of the variables 
measured in the radar environment. 48 That this analysis used a different set of predictors for group membership (as compared to the regression models) may have practical benefits. As can be seen in Table 15, all of the variables can be measured using commercially available aptitude tests. It might be more efficient to improve the present selection battery by applying these standardized measures rather than by refining the picture assessment test (although this might also be useful). However, the selection of a valid success criterion would be essential in the creation and testing of any assessment battery. The importance of predictor variables can be highly dependent on the nature of the outcome measure. It may also be important to select controllers based on their hemispheric cognitive style.

Considerable research would have to be conducted to assess the predictive validity of any new selection measures.

The results of this study do not supply conclusive evidence for or against perspective or three-dimensional displays as opposed to the current plan view radar presentation. However, giver that aircraft altitude appears to be an important element of the picture, it should be prominently displayed in the data block. The readout of speed, however, could assume a less obvious position. Given the suggestion that visual/holistic cognitive style may be more predominant in the radar environment, computerized work aids should focus on providing graphical as opposed to verbal or numerical information. For example, a conflict prediction system should use graphics for showing projections of aircraft position and visual coding of potential problems rather than written warnings about an impending loss of separation. In order to help the controller deal with increased workload in both the procedural and radar work situations all relevant aircraft data should be presented so that working memory (or the picture) is not cluttered with unnecessary facts and can be oriented toward problem solving.

48 However, the number of subjects was not sufficient for reliable multivariate procedures and so the discriminant function results must be considered as tentative. 


\subsection{Suggestions for Further Research}

The Preliminary Study pointed to further research which would be worthwhile. Most interesting of these would be to explore the potential of cluster analysis for looking at how the controller organizes screen information. With more realistic screen presentations and a larger variety of aircraft and traffic situations much more information could emerge. In addition, another technique, multidimensional scaling, could be used with the same data to explore the air traffic controller's conceptual space and knowledge structures.

The finding that experienced controllers tend to think in dichotomies (as evidenced by their similarity judgements) is supported by the comments of many Air Traffic Services personnel interviewed during this research. This suggests that a particular perceptual or decision-making style may be necessary for air traffic control. It is probably not helpful to spend time making fine distinctions in a job which requires quick evaluation followed by decisive action. It would be useful to work out or search for methods for testing this cognitive style and exploring whether it is an aptitude trainees bring to school or whether it is developed there (or both).

Experiments 1 and 2 offered evidence that the controller's picture is important for good air traffic control. However, there are other levels of the mental model which were not touched upon by the picture assessment. For example, it would be interesting to discover how well trainees understand the meaning of the air traffic picture they are maintaining and whether this comprehension is important. The skill for prediction could also be investigated using a similar approach. Following further research of this nature it would be important to work with experienced controllers to determine if these findings generalize outside of the simulation environment. However, the difficulty in locating sufficient numbers of subjects and establishing criterion measures of controller expertise are problems which would have to be overcome. 
A special simulation program on a microcomputer might be a more efficient means for measuring the picture. Once this was developed, a large number of trainees could be tested and predictive validity for success in school and in the field could be assessed. If this work was successful, an automated computer-based testing instrument could be developed and provided to a applicants as an experimental selection test. Further studies would then t:ave to be completed on its effectiveness as a selection tool.

There are suggestions in Experiments 1 and 2 that controller trainees approach radar and procedural air traffic control tasks with ingrained cognitive styles favouring verbal/analytic or visual/holis ic problem solving. It is possible that each cognitive style is effective in air traffic control. However, there are indications that while non-verbal abilities may be useful for radar control, they are not so successful when applied to procedural control. Further research on controller characteristics should include some way of identifying and measuring cognitive style and its relevance to air traffic control.

For example, studies of the form of the controller's picture might be more productive if subjects were assessed for cognitive style prior to experiments on the form of the picture. This could be achieved through the use of such instruments as the Visualizer-Verbalizer Test (Richardson, 1977) or the Word-Shape Sorting Test (Galin \& Ornstein, 1974). The existence of these differ.ices in the trainee population could then be confirmed. Those with a more visual approach could be examined as a separate group and their data would not be affected by results from subjects who prefer verbal problem solving.

Another interesting area for further research would be to look at the role of the "executive" in allocating memory resources (Hitch, 1987). The importance of good management of cognitive capacity has also been identified in studies of selective attention with regard to display sampling (Gopher, 1982). However, there is little information on how to improve this hypothetical entity. 


\subsection{Conclusions}

It is impossible to study the nature of mental models without considering memory. "The role of working memory is... a crucial determinant of the ability to set up a mental model" (Manktelow \& Jones, 1987). Long term memory also must be included as the storehouse of knowledge which supports the central executive's management of attention and memory and which offers information on the static details of the system. Moray's (1979) comment that "Models are learned, not remembered" (p. 34) suggests that skilled air traffic controllers acquire a complex knowledge base about the domain through education and practice. They have reached the "autonomous" stage of skill acquisition "during which the component processes become increasingly automatic, less subject to interference from other tasks, and less available to conscious awareness" (Hammond, 1987, p. 169). Only a part of this information is in awareness at any given time; it is this element which has been labelled the "picture" during this research project.

Irrespective of the role of memory in maintaining the mental model, it is difficult to avoid employing a memory paradigm for exploring it. However, care must be taken when designing experiments to take into account the limits of short term memory when asking subjects to recall information. The less time assessment techniques require, the closer the data will be to what the air traffic controller is thinking about. Brief probes for information without interrupting a task simulation would be a way of avoiding problems with the decay of information in memory. Techniques which use comparisons of stimuli (such as cluster analysis and MDS) move away from recall techniques and hold promise for uncovering some hidden dimensions of mental models.

There are a number of findings from this research which suggest that cognitive styles are an important factor in air traffic control. Such dimensions as impulsive-reflective and verbal/analytic-visual/holistic have helped to explain some of the data. If these or other cognitive strategies are shown to be factors in trainee performance it will be necessary to 
decide whether candidates should be sought out with a specific orientation or whether ATC training or tasks can be modified to accommodate more than one type. Egan and Gomez (1985) outlined a methodology to assay, isolate, and accommodate individual differences in human-computer interaction which is designed to change or eliminate those tasks which cause learning difficulties. Such an approach might increase the chances for success in training for many students.

The differences in cognitive strategy between controllers may also explain the mixed or inconclusive results obtained during this research with regard to spatial ability and the presence of two- or three-dimensional visualization. It may be that some controllers do visualize in 3D while some do not. Further research might identify any advantages of one approach over the other.

The understanding of the air traffic controller's picture which can be pieced together from the foregoing research is that it is to a large extent resident in short term or working memory. Although there may be task-related information which passes through consciousness that does not result in an entry in the visuo-spatial scratchpad or articulatory loop, it seems reasonable to assume that it is only required for immediate activities and is promptly discarded. The concept of the picture (as the word implies) is that it is an entity with some consistency over time. Figure 8 shows the relationship between the controller's knowledge base, the picture, and moment-to-moment awareness which has been suggested by the present research.

The knowledge base is a complex array of information which is largely unconscious. The picture is a "holding area" (or supplementary display) where images and verbal data are held for ready use. "Momentary awareness" is the arena of ongoing conscious processes. It may contain sensory information or cata from the knowledge base or picture but there is no storage, only the ongoing stream of consciousness. Data from auditory or visual displays may be momentarily perceived, neld in the picture, and also may update the 


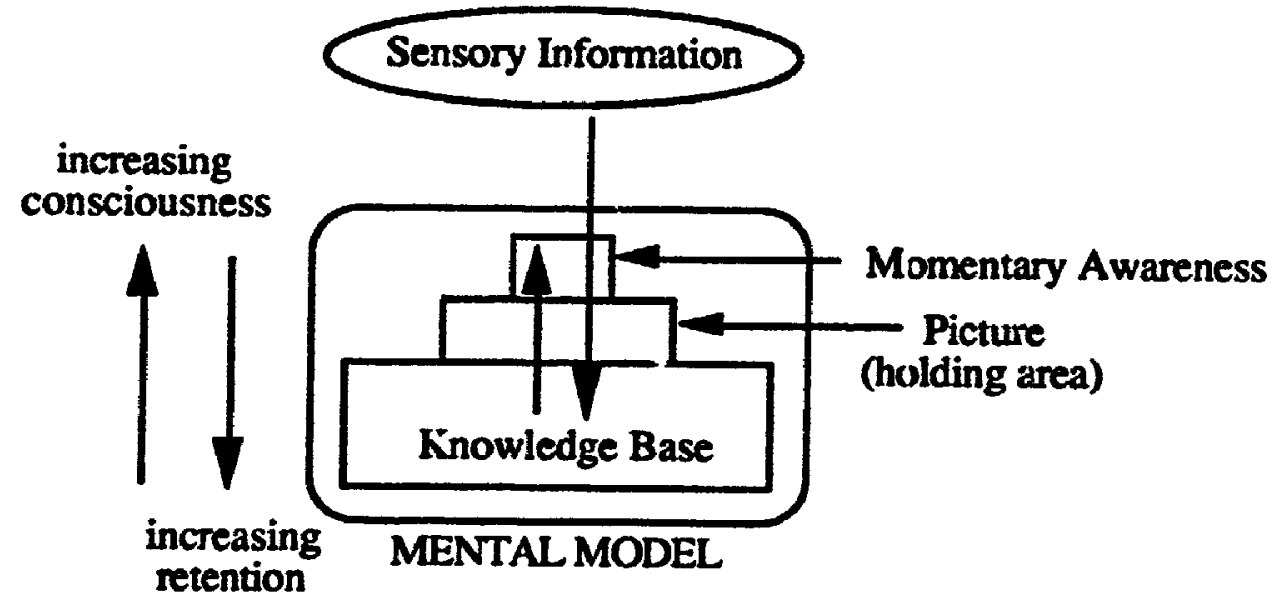

Eigure 8 . The relationships between the air traffic controller's mental structures. 
knowledge base if there are long term implications. Information in the knowledge base influences and structures the picture and directs momentary awareness. A relevant fact or rule may also emerge from this repository for storage in the picture or for use in a momentary activity. As noted by the two arrows to the left of the diagram, there is generally more awareness as information moves up through the system although it is retained for a shorter period of rime.

A direct perception (Gibsonian) approach to the work of the air traffic controller would not support the development of information processing submodels to describe hypothetical cognitive structures. In addition, it could be claimed that the levels of the mental model shown in Figure 8 correspond with areas of memory which have been identified and researched by other authors. However, this model of the controller's internal representations is useful for organizing thinking about the issues discussed in this paper and serves as a way of focusing on a user's interaction with a specific system. Understanding that this research has been focused on the picture, the following statements can now be made about it:

1. It contains specific kinds of information which are useful for managing aircraft.

2. It is important to include the correct elements in the picture given the limits on how much can be retained; keeping the wrong information in the picture can interfere with task performance.

3. The contents of the picture are dependent upon the work environment.

4. The picture may serve the function of holding important information which is not readily available to the controller from the human-machine interface.

5. Experienced controllers can maintain a static image of the radar screen for about nine seconds before updating it.

6. The picture can contain at least 22 items of accurate information about aircraft (some controllers are able to retain up to 35 items). 
7. For some individuals, the picture seems to have three dimensions (altitude is coded visually).

8. Subjects may expand their visual image of the altitude dimension of the picture.

9. Good retention of the picture appears to support efficient error detection.

These explorations of the air traffic controller's mental constructions have been both challenging and frustrating. Nevertheless, the findings in the above list and the other results reported here suggest that the study of the mental model san have rewarding theoretical and practical results. As in any scientific effort, each stage of this work has evolved better and more specific questions. This process has been extremely educational with regard to the domain and with reference to the conduct of applied psychological research in general. Hopefully there will be opportunities to pursue it further. 
Appendix A

Details of Preliminary Study 


\section{A - 1. Introduction}

Mental models can be considered in terms of their form, structure, and parameters (Rouse and Morris, 1986). The Preliminary Study incorporated a number of ways of exploring the form of the air traffic controller's picture (sensory code or spatial dimensions), how the information contained in it is organized or structured, and its parameters (amount of data, resiliency, or accuracy). In addition, because one of the purposes of a mental model is to help predict the behaviour of a system, assessments of controllers' ability to project the future positions of aircraft and to detect unexpected changes in their movement were also included. In each experiment, experienced controllers were contrasted with novices with the aim of identifying unique skills.

\section{A-1.1 Form}

As discussed earlier, there is an ongoing debate in the literature on mental models regarding the underlying form of representation of the construct. A primary concern is whether it is verbal (propositional) or spatial in nature. It can be assumed that the cognitive representation of air traffic situation is comprised of a mixture of these formats simply because the information available at the system interface is presented spatially, numerically, and in words. For example, aircraft $x, y$ position is shown on a map while altitude is displayed as a number. Flight strips are arranged following a rough geographic metaphor although the intended track of the aircraft is described by fix abbreviations (such as "DAVNI") and numbers.

It is not the intention here to sort out all of the data available to the controller and determine how it is being cognitively represented but to focus only on altitude. It would be useful to know whether the third dimension of an aircraft's position is mentally portrayed as a numeric quantity (as displayed on the screen) or whether it is converted into a spatial image. Although altitude can be represented in either way, it is possible that air traffic controllers prefer one format over the other given the tasks they must perform. An answer 
to this question could influence whether future radar displays should retain a plan view format or show some type of perspective or even true three-dimensional scene.

One approach to operationalizing this problem would be to modify the method used by Pinker (1980) to study tlie visualization of three-dimensional arrays of objects. Pinker (1980) asked subjects to view plastic shapes suspended on fishing line in a box, to memorize their positions, and then to mentally estimate interobject distances. He wanted to determine if the subjects' estimates of interobject separation indicated a two- or threedimensional internal image.

To study the controller's image the two-dimensional information on the radar screen could be used. In this case the third dimension (altitude) is numerically coded. If controllers conceptualize this display of aircraft in three spatial dimensions their judgements of interobject distances should correlate better with the three-dimensional separations of the aircraft than with the two-dimensional distances between them (as found in Pinker's, 1980, results with the plastic shapes).

To illustrate, if $A$ and $C$ in Figure $A-1$ is the side view of two aircraft, the distance between $A$ and $C$ will be affected by the altitude of $C$. If $C$ is on the same level as $A$ their separation (line $\mathrm{AC}$ ) will be less than if $\mathrm{C}$ is higher than $\mathrm{A}$ (line $\mathrm{AC}$ ). If a controller has a two-dimensional visualization of two aircraft at differing altitudes (in $x$ and $y$ with altitude verbally coded), line AC will represent their separation. However, if altitude is conceived of spatially, line $A C^{\prime}$ will be visualized as their separation.

During an air traffic control simulation the subject could be asked to make interaircraft distance estimates between each pair of targets by drawing a line representing their relative separation. If the judgments correlate better with three-dimensional interaircraft separations this would suggest that the traffic is being visualized in three dimensions. 


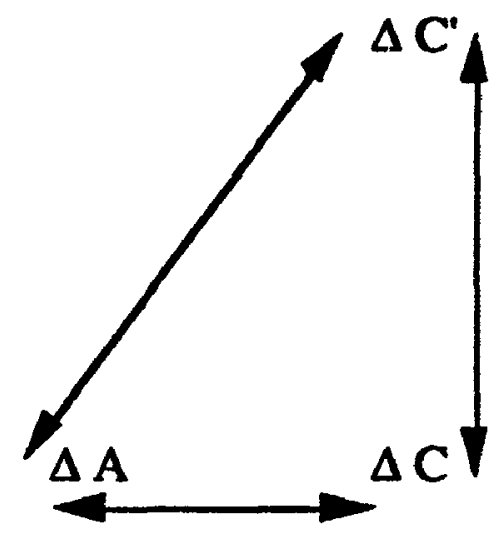

Figure A-1. The trigonometry of Pinker's (1980) mental imagery experiment (as applied to air traffic control). Line $A C$ is the separation between two aircraft at the same altitude. Line $A C^{\prime}$ is the aircraft separation when an altitude difference exists or is visualized. 
However, there are some problems with this approach. A good correlation of judgements with three-dimensional interaircraft separations would not necessarily prove that altitude is being visualized spatially. It might be that the subject converts the horizontal separation into a quantity and estimates the length of the hypotenuse employing mental trigonometric calculations. However, it is probable that this would take longer than converting the altitude quantity into a spatial dimension and then making an estimate of the interaircraft separation by visualizing the spatial vectors. It would also be a formidable feat of mental arithmetic.

Another difficulty with this method emerged in discussion with an experienced air traffic controller. He observed that, if asked to make an interaircraft distance estimate, he would normally represent this by the two-dimensional separation. This is what is clearly represented on the screen. When checking the separation of two aircraft he said it is likely that the controller first compares the numerical altitude data available on the radar display to check for an altitude difference. If this indicates satisfactory separation no further work is necessary. If the altitudes are the same or are converging, further steps must be taken to check flight path, speed, etc. If the two aircraft are in danger of losing separation and action must be taken it is only at this point that a visualization of the spatial relationships may be helpful.

An additional problem with the trigonometric approach is that it does not hold up well in the environment of air traffic control. Pinker (1980) was able to arrange his laboratory stimuli so that there would be a significant difference between the two-dimensional and three-dimensional interobject distances. Referring back to Figure A-1, it can be seen that the difference between lines $A C$ and $A C$ increases with the height of line $\mathrm{CC}^{\prime}$. However, because of the scale of dimensiors used in air traffic control, line $\mathrm{CC}^{\prime}$ (or the altitude) will actually be quite short as compared to AC or horizontal distance. 
On a radar display, aircraft may be from three to thirty miles apart horizontally (depending upon the range) but only 1000 to 29,000 feet ( 0.2 to 4.8 miles) apart vertically. Therefore, in many of the trigonometric solutions for pairs of aircraft at different altitudes tine base of the triangle (AC) will not be significantly shorter than the hypotenuse (CC'). In these situations, the two- and three-dimensional interobject distances will themselves be well correlated rendering Pinker's (1980) approach useless.

These problems with using the estimation of interobject distances suggest that another way to test a subject's spatial imagery should be considered. An alternate approach could be to create the conditions where three-dimensional imagery will most likely be used and then to ask subjects to build a physical three-dimensional model of the situation. A simulation with two conditions could be designed. In one case, two aircraft approach each other with no chance of conflict. In a second condition it is obvious that a loss of separation could occur. The simulation is interrupted and the subject is asked to plot the present and future positions of the aircraft on a three-dimensional plotting board.

If the subject is using three-dimensional spatial imagery to visualize the potential conflict situations, it would be predicted that the positioning of the markers on the plotting board will be both more accurate and rapid as compared to instances where no hazard exists. Subjects with extensive experience in air traffic control should also show superior skills as compared to novices. Although future positions could be mentally calculated from the ground speed and climb rate data provided by the simulator, the subject would have to demonstrate the result in a purely spatial format (using a plotting board and markers). This approach is also based on the assumption that controllers would focus their spatial skills on the potential conflicts rather than on the safe situations.

During the development of the experiments it was observed that subjects expanded the altitude scale when conceptualizing an airspace. For example, on the map used for subject responses, an altitude of 20,000 feet would equal $25.5 \mathrm{~mm}$ above the map if 
measured consistent with the map's $x, y$ scale (where $7.75 \mathrm{~mm}=1$ nautical mile $(\mathrm{nm})$ ). However, most subjects indicated a much higher level when asked to point to a level of 20,000 feet above the map indicating they did not visualize altitude to scale.

This finding allowed Pinker's (1980) trigonomeuric approach to be applied after all. If subjects visualize an expanded third dimension, it would create a much greater difference between the two- and three-dimensional interobject distances. Therefore, subjects' relative interobject distance judgements might yet correlate neaningfully with a two- or threedimensional perception of an array of aircraft. Therefore, both model building and interobject distance judgements were employed in the pilot study.

\section{A-1.2 Structure}

Another aspect of mental models which is worthy of exploration is their organization or structure. Of interest is the relative importance of aircraft and the basis upon which the controller organizes them. A recall task was used to investigate the first question. It was hoped that the order in which aircraft were remembered would provide clues as to their importance in the mental model. A cluster analysis based on similarity judgements between aircraft was employed to supply information on how cuntrollers group targets.

\section{A-1.3 Parameters}

What kind of information can be gathered about the picture with regard to capacity, resiliency, or accuracy? The recall task outlined above would provide data on the number and accuracy of aircraft attributes in the picture. As previously discussed, it was observed that subjects tended to expand the altitude scale when referring to airspace maps.

Therefore, a formal test was included in which subjects were asked to point to a level above the sector map which corresponded to 20,000 feet in their mental image of the airspace. This task was designed to provide some impression of the subjective dimensions of the picture as well as to explore the variability of such judgments. 
It can be assumed that the mental model must be updated from time time because of the natural fading of the information in working memory, retroactive inhibition, and a constantly changing stimulus situation. An opportunity to observe updating was involved while the subject was included in plotting tasks. During the phases of the experiment which included three-dimensional model building, the number of times the subject glanced back to the screen was counted. It was assumed that this behaviour could be an indicator of the resiliency of the subject's picture. It should be noted that the display was "frozen" during these tasks which eliminated the need to track moving aircraft.

\section{A-1.4 Predicting Aircraft Location}

As previously mentioned, one application for a good picture of air traffic mentioned by Whiffield (1979) and Rouse and Morris (1986) is that it supports prediction of the future behaviour of the system. Further plotting trials were added to examine controllers' ability to predict future aircraft position. An additional aim was to study the differences between predicting the positions of aircraft on a predetermined routes (overflight) versus those which were under active control (arrivals being vectored for landing).

\section{A-1.5 Fault Detection}

Given that the hypothesized picture or mental model has the purpose of generating expectations about system behaviour, this should allow the detection of errors (Rouse \& Morris, 1986; Norman 1986). To explore this issue, it was decided to include opportunities for the subject to detect abnormal aircraft actions in simulation exercises.

\section{A-1.6 Hypotheses}

A primary assumption in the Preliminary Study was that the air traffic controller, faced with the task of separating aircraft, creates a mental model it in order to assist with the work. It was expected that veteran air traffic controllers would demonstrate a more developed and accurate picture as compared to novices. Therefore, it was predicted that 
controllers would have superior and more uniform results or unique skills as compared to non-controllers. The specific hypotheses for the controller group were that it would show:

1. Task-related priority of recall of aircraft.

2. Better recall performance in terms of rate, amount, and accuracy.

3. Prioritization of recall of aircraft attributes in line with previous findings (Bisseret, 1970; Means, et al., 1988).

4. A common, more refined, and task-related basis for the clustering of targets into groups.

5. Correlation of interobject distance judgments with a three-dimensional spatial image.

6. Higher, more consistent and more uniform subjective ceiling judgments.

7. Fewer head turns to check the TRACON screen during plotting tasks.

8. Better response time and accuracy with plotting tasks.

9. More accuracy in predicting arrivals.

10. More accuracy in predicting conflicting (as opposed to non-conflicting) aircraft positions.

11. Better fault detection.

However, it was recognized that the predictions listed above might not be as clear cut as might be assumed. It is probable that the ability to form a workable picture is based upon aptitude and then enhanced by training and so some of the non-controller subjects would be expected to show results in line with some of these hypotheses. However, the controllers as a group should show these abilities more consistently. If controllers prove superior to all naive subjects in a particular experiment, the task in question is probably heavily dependent on training and experience. However, if most subjects show a common pattem of results, it may indicate a typical human response more which is more reliant on native aptitude. 


\section{A-2. Method}

\section{A-2.1 Subjects}

Subjects were recruited from three sources. The most readily accessible personnel were those working as "pilots" (simulator operators) at the Transport Canada Air Traffic Services Research and Experimentation Center (four were recruited, average age $\mathbf{2} 28$ ). Experienced controllers were more difficult to find. Two retired controllers, one controller who was being retrained, and two instructors from the Transport Canada Training Institute were available (average age $=51$ ). In addition, three undergraduate students from Carleton University (average age $=22$ ) were sought out for to their presumed lack of knowledge about air traffic control.

\section{A-2.2 Apparatus}

Air traffic control exercises were created using a program called TRACON (Wesson \& Young, 1988) running on IBM-PC or compatible. It was decided to use this computer simulation for the sake of expediency and control. The main simulation system was in frequent use during the inception stages of this study and much more time would have been required to program experimental exercises and run subjects. Versions of TRACON are in use in various air traffic control research projects such as at NASA Ames. It was considered to be an acceptable simulation for the purposes of this study by the experienced controllers who used it.

A screen printout of the TRACON interface can be seen in Appendix B.4.2. Electronic flight data strips were provided in addition to the radar display and a "communications" section appeared below the radar screen. The aircraft were depicted by small aircraft icons. A data block was attached to each target showing present altitude and, if height was changing, goal altitude and the direction of change (climbing or descending). Speed was not shown in the data block but planned speed was included (along with route information) on the electronic flight strips. 
TRACON was run on an IBM AT with an EGA colour monitor. In addition, there were two other colour monitors "daisy chained" together. The experimenter acted as a pilot and entered commands to the simulator through a computer keyboard. The subject sat in front of a second monitor and issued verbal commands to the pilot, much as is done on the main simulation system. On the subject's right was a sector map taped to a piece of pressboard (acoustic ceiling tile) on which responses were made. "Plotting markers" for the three-dimensional modeling and prediction tasks were created from knitting needles and appropriately sized beads ("sliders"). Timing was done manually with a stopwatch.

A subject's work on the map and the simulation were videotaped using two separate cameras and VCRs so that data could be collected on subject behaviour and on the simulation exercise concurrently. After each experimental trial, a transparent plastic sheet was placed over the screen of a 21 inch monochrome monitor and transparency markers were used to trace the locations of aircraft for scoring purposes. These positions were coded as $x, y$ screen coordinates for data entry.

Fight plans and a Toronto sector radar map were created by editing files in TRACON. The Toronto sector background was similar to that used on the main simulation system but was simplified by leaving out many of the altitude zones and other graphics which would have cluttered the smaller screen. Five flight plans were created for the various conditions of the experiment. They ran for 30 to 40 minutes each. Workload was regulated so that no more than 9 or 10 aircraft were on the screen at once, one-third being overflights and not requiring active control.

\section{A-2.3 Procedure}

All experimental conditions were rin using the Toronto sector. The tasks of the controllers at this airport are divided so that some controllers handle arrivals and overflights while others are responsible for departures. The sector is further divided so that controllers handle different runways and sometimes may also be segregated into those who control 
low versus high arrivals. Controller activities in this study were restricted to the control of arrivals to two runways and the monitoring of overflights over the sector.

Table A-1 outlines the experimental tasks and expected outcomes for the preliminary research. The experimental protocol for the Preliminary Study can be found in Appendix B.4.1.

\section{A-2.3.1 Practice Exercise}

After introducing the subject to the experiment and describing the tasks a 30 minute exercise was stanted to provide experience with the system. The experimenter emphasized that proper procedures and terminology were to be observed where possible but were not critical for the purposes of the experiment.

\section{A-2.3.2 Recall Exercise}

After the practice run another exercise, similar in type and length, was initiated. At the outset the subject was informed that this run would be stopped after about 20 minutes and the CRT would be turned off. The subject was instructed to then turn to the right and write down all remembered aircraft information on a sector map (aircraft location, heading, identifier, altitude, and speed).

The exercise was run ahead until a particular set of nine aircraft were on the screen. The simulation was paused and the subject's monitor was turned off. The subject was videotaped while working at the recall task and the tape was later reviewed for order of recall and response times. After the experiment, actual aircraft locations and other data were taken from the videotape playback of the TRACON exercise.

After the subject had completed this task, the monitor was turned back on and pairwise comparisons of all of the aircraft on the screen were made. Following this, the subject was asked to draw horizontal lines representing (in relative terms) how far apart each aircraft was from every other aircraft. 


\begin{tabular}{|l|l|}
\hline \multicolumn{1}{|c|}{ EXPERIMENTAL TASKS } & \multicolumn{1}{c|}{ OUTCOMES } \\
\hline Recall of Screen Targets & $\begin{array}{l}\text { Odrt } \\
\text { Rate } \\
\text { Accuracy }\end{array}$ \\
\hline Target Similarity Judgements & Grouping of Targets \\
\hline $\begin{array}{l}\text { 3D Spatial Judgements: } \\
\text { (a) Interobject Distance } \\
\text { (b) Height of Ceiling } \\
\text { (c) Present and Predicted Positions }\end{array}$ & 2D or 3D Distance Correlations \\
& $\begin{array}{l}\text { Altitude Expansion Factor } \\
\text { Response Time } \\
\text { References to Screen } \\
\text { Accuracy }\end{array}$ \\
\hline Fault Detection & $\begin{array}{l}\text { Number Detected } \\
\text { Response Time }\end{array}$ \\
\hline
\end{tabular}

Table A-1. The experimental conditions included in the Preliminary Study. 


\section{A-2.3.3 Subjective Altitude Judgements}

Casual observations had indicated that people expand thes altitude scale when working with sector maps. Subjects were asked to view a copy of the map used for the recall task and to point to a level above it which represented an altitude of 20,000 feet. The average height over three trials was calculated.

Another set of trials was then initiated using one of the plotting markers. One marker was positioned in the centre of the sector map and the slider was moved to the top. Subjects were told that, although the previous estimates of altitude had been made on a free basis, they should rescale their judgements so that the top of the marker was considered to be at 20,000 feet. Subjects were then given a series of altitudes to judge $(1000,5000$, 10000 , and 15000 feet) and asked to move the slider to the level corresponding with each altitude. The consistency and linearity of their responses were checked.

\section{A-2.3.4 Practice Plotting Exercise}

The use of the plotting markers to show present, two minute, and five minute positions was demonstrated. Subjects were then asked to plot the present and future positions of aircraft using the plotting markers and sector map. The TRACON exercise was started and paused periodically to allow the subjects to practice plotting the present and future positions of two single aircraft (one in level flight and one changing altitude) and two aircraft on crossing tracks. Subjects were encouraged to check the accuracy of their predictions as the exercise proceeded.

\section{A-2.3.5 Modeling and Prediction of Overfining Aircraft and Arrivals}

The next experimental condition involved another 30 to 40 minute air traffic control simulation very similar in task demands and workload to the recall exercise. The system was paused at preplanned poirts and the subject was asked to plot the present and future positions of aircraft. The first two targets were single aircraft on overflights; one was in level flight and the other was climbing. The present and two minute positions were plotted. 
The third and fourth trials included pairs of arriving aircraft; the subject was required to plot their present, two, and five minute positions. 49

The experimenter, after pausing the TRACON program in each instance, moved over to the table where the sector map was located and gave the subject the names of the aircraft to be plotted. The controller's monitor was left on and the subject was allowed to refer to it as often as needed. Head turns were counted as was the time the subject required to plot the specified aircraft. After the markers had been positioned, the subject then wrote aircraft identification and altitude (present and predicted) (n the map with coloured pens corresponding to the present, two, and five minute positions.

The experimenter also recorded the time at which the system had been stopped and the altitudes of the specified aircraft. The actual present and future positions and altitudes were retrieved from the TRACON videotape after the experiment had been completed.

\section{A-2.3.6 Modeling and Prediction of Intersecting Aircraft}

The final simulation exercise involved plotting the present and future positions of overflying aircraft on intersecting courses (crossing tracks, overtaking, or head-on). In three of the five trials, the aircraft were in danger of losing separation (but not colliding) and in the other two trials, there was no danger of conflict. In each case the two aircraft entered the sector on intersecting courses. At a predetermined time, one of the aircraft made an altitude change which would either create a conflict or result in ample separation. Shortly after this, the exercise was paused and the subject was asked to plot the present and future positions of both aircraft.

49 In most of the experimental trials the plotting of the five minute position was omitted. In all trials where aircraft were changing altitude they reattained level flight close to the two minute future position. It was decided that plotting the five minute position would not add much information and would needlessly lengthen the experiment. 
As in the previous exercise, the experimenter recorded head turns, time to plot aircraft position and (from the TRACON videotape) the actual present and future positions and altitudes of the targets.

\section{A-2.3.7 Fault Detection}

Subjects were forewarned that two pilot errors would be injected into each of the three experimental simulation exercises. Errors were made on either overflights or arrivals, were either immediate or delayed, and involved either altitude or heading. The time required for the subject to detect the error by commenting on it in some way was recorded by the experimenter.

\section{A-3. Results and Discussion}

\section{A-3.1 Recall Order}

Given the possible sources of variance present, the goal was to demonstrate that an aircraft's importance in the simulation exercise (as judged by independent raters and the subjects) corresponded to its frequency of occurrence in the first five aircraft recalled. If this could not explained by the effects of stimulus duration or recency it could be assumed that the elements of the picture had a task-related weighting in memory which influenced their recall order.

A count of the frequency of occurrence of the nine aircraft present on the display in the first five recalled is shown in Figure A-2. At the top of the list (with high emphasis for all three subject groups) were AAL88, KLM850, and CFGM. USA909 was recalled 50\% to $60 \%$ of the time while TOR506, CDN541 and ONT670 were remembered between $20 \%$ and $70 \%$ of the time, depending upon subject group. ACA250 and TGO342 did not come up often, except that the pilots recalled ACA250 over $70 \%$ of the time. 


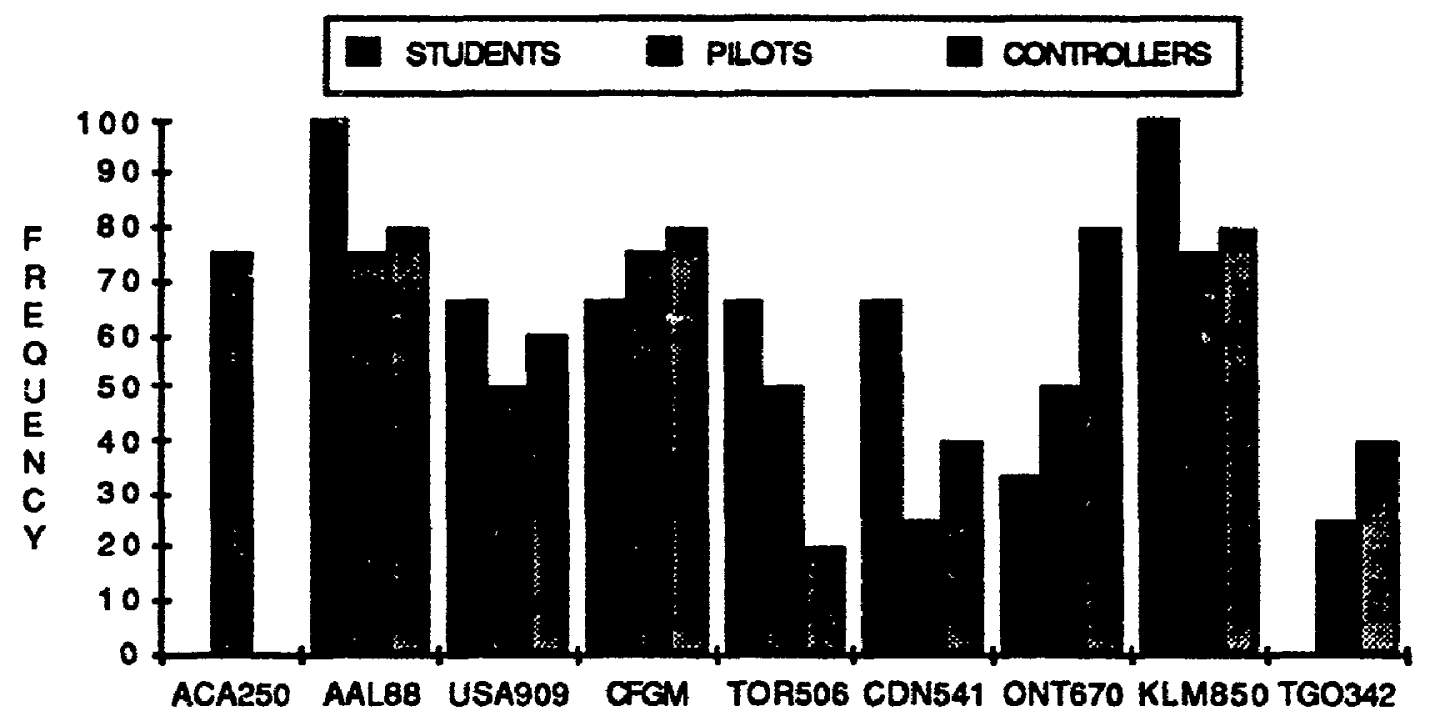

Figure A-2. Frequency of recall (in per cent) of aircraft in first five targets recalled. 
Based to these frequency counts the aircraft might be roughly ordered into four groups across all subjects:

1. AAL88, KLM850, CFGM

\section{USA909}

\section{TOR506, CDN541, ONT670}

\section{ACA250, $1 \mathrm{GO} 342$}

Referring to the sector map (see Appendix C-4.1), it can be seen that KLM850 and AAL88 were the next aircraft to land and so could required considerable attention. CFGM was a low and slow overflying aircraft. Because it had been in proximity to CDN541 and AAL88, and because it could have been a problem for other arriving aircraft, it had to be watched. USA909 was to be handed off to the next sector controller in a short period of time. TOR506 and CDN541 were next in line for arrival and ONT670 may have been important because it was passing over the runway area. ACA250 in most cases had already been handed off to Toronto tower and TGO342 was flying over the secto:" $\mathrm{H}$ : a high altitude and would have posed little threat.

From the above qualitative analysis, it appears that the frequency of recall of the nine aircraft in the first five has some correspondence to their importance in the task. The Zeigamik effect (Stevens, 1966) could also be operating in that targets associated with unfulfilled tasks (such as arriving aircraft which had not yet been handes. off to the Toronto tower) may have been remembered better than aircraft for which all controller iasks had been completed.

In the chart in Figure A-2, the aircraft are shown in order of appearance in the exercise. The leftmost aircraft had been on the screen for about twelve minutes arit the rightmost aircraft had just entered about one minute before the simulation was paused. Any recency or exposure effects should show up on the chart as an increase or decline of recall 
frequency (respectively) across the chart (depending upon which is operating). From inspection of Figure A-2, it appears that neither of these effects is obviously present.

As an independent check on these findings, five licensed controllers were asked to rank the aircraft in the air traffic control situation shown in Appendix C-4.1 in terms of importance. In addition, the twelve participants in the experiment were contacted by mail and were also asked to prioritize the aircraft. A count was made of the frequency of occurrence of the aircraft in the top five for both independent raters and subjects. The aircraft were then ranked by frequency of recall in each group (students, pilots, and controllers) and a Kendall rank-order correlation was completed between the recall results and subjects' and independent raters' rankings of aircraft importance (see Table A-2).

It is evident from this table that the independent judges' rankings of aircraft importance correlated strongly and significantly with each group's order of recall data. In addition, subjects' own importance rankings matched well with their order of recall (except for the controller group). Rankings of importance between groups were more highly correlated than were the between-group relationships in order of recall. Controllers' order of was not significantly different froni the other groups.

Perhaps the fact that subjects' importance ratings were completed a few weeks after the experiment had a negative effict on their correlation with recall frequency. The observation that the ccrrelation . of recall order with importance were highest for students, lower for pilots, and lowest for controllers suggests that the importance ratings may have been difficult for the more experienced subjects when taken out of the context of a simulation and presented at a later time on paper. These results did not support the prediction that controllers had a higher degree of task-related prioritization of recall but indicated an interesting pattern of behaviour. 


\begin{tabular}{|l|l|l|l|l|l|l|l|l|l|}
\cline { 2 - 10 } \multicolumn{1}{c|}{} & All Ss & I- All & Stud. & I- Stud. & Pilots & I- Pilots & Cont. & I- Cont. & I-Indep. \\
\hline All Ss & 1.00 & & & & & & & & \\
\hline I-All & 0.44 & 1.00 & & & & & & & \\
\hline Stud. & 0.89 & 0.83 & 1.00 & & & & & & \\
\hline I-Stud. & 0.50 & 0.83 & 0.78 & 1.00 & & & & & \\
\hline Pilots & 0.78 & 0.44 & 0.72 & 0.56 & 1.00 & & & & \\
\hline I-Pilots & 0.61 & 0.94 & 0.94 & 0.78 & 0.61 & 1.00 & & & \\
\hline Cont. & 0.94 & 0.22 & 0.78 & 0.40 & 0.61 & 0.39 & 1.00 & & \\
\hline I-Cont. & 0.39 & 1.00 & 0.78 & 0.83 & 0.50 & 0.94 & 0.22 & 1.00 & \\
\hline I-Indep. & 0.78 & 0.94 & 1.00 & 1.00 & 0.78 & 0.94 & 0.72 & 0.89 & 1.00 \\
\hline
\end{tabular}

Table A-2. Kendall rank-order correlations between of order of recall and ratings of importance. The nine aircraft were ranked by frequency in the first five recalled; combined data for all subjects and the three separate groups (students, pilots, and controllers) are shown. The "I" columns are each group's rating of the importance of the aircraft. The "I-Independent" data are ratings of importance by an independent group of controllers. Correlations between recall order and importance are printed in bold. T values of 0.45 or over are significant at p<.05 (Siegel \& Castellan, 1988). 
In spite of the curious result regarding the controller rankings, there is evidence from the qualitative and quantitative analysis of this data that the order of recall of aircraft is related to importance. Thus it is reasonable to assume that the information present in a subject's picture (or that which is remembered most vividly) is related to the degree of criticality it has for ongoing tasks.

\section{A-3.2 Recall Rate and Accuracy}

Although between-subject variance in speed of recall mitigated against the appearance of clear between-group differences, it was evident that three of the controllers were quite fast (see Figure A-3). However, two of the controllers were as slow or slower than the non-controllers. In one case this seemed to be due to low recall efficiency (C4 only recalled four out of nine aircraft as seen in Figure A-5). Subject C2 took an excessive amount of time to draw the last target remembered and this artificially reduced the recall rate. Figure A-3 should be considered only as a rough measure of recall speed because subjects were told to emphasize accuracy and the method of timing proved not to be ideal for the purposes of these analyses. A t-test for differences between controllers and noncontrollers was not significant. However, when subjects $\mathrm{C} 2$ and $\mathrm{C} 4$ were removed as outliers a significant time difference emerged ${ }^{50}$ although controller speed was still not more consistent. 51

With regard to accuracy, it is quite clear from inspection of Figures A-4 and A-5 that controllers had better recall of target position as measured by absolute and ballpark error methods (see Table A-3 for the rules used to calculate ballpark accuracy). However, presumably due to the small number of subjects, unequal sample sizes, and variance of the

${ }^{50}$ See Appendix C-4.2 for t-test data.

51 See Appendix C-4.3.A for ANOVA data. 


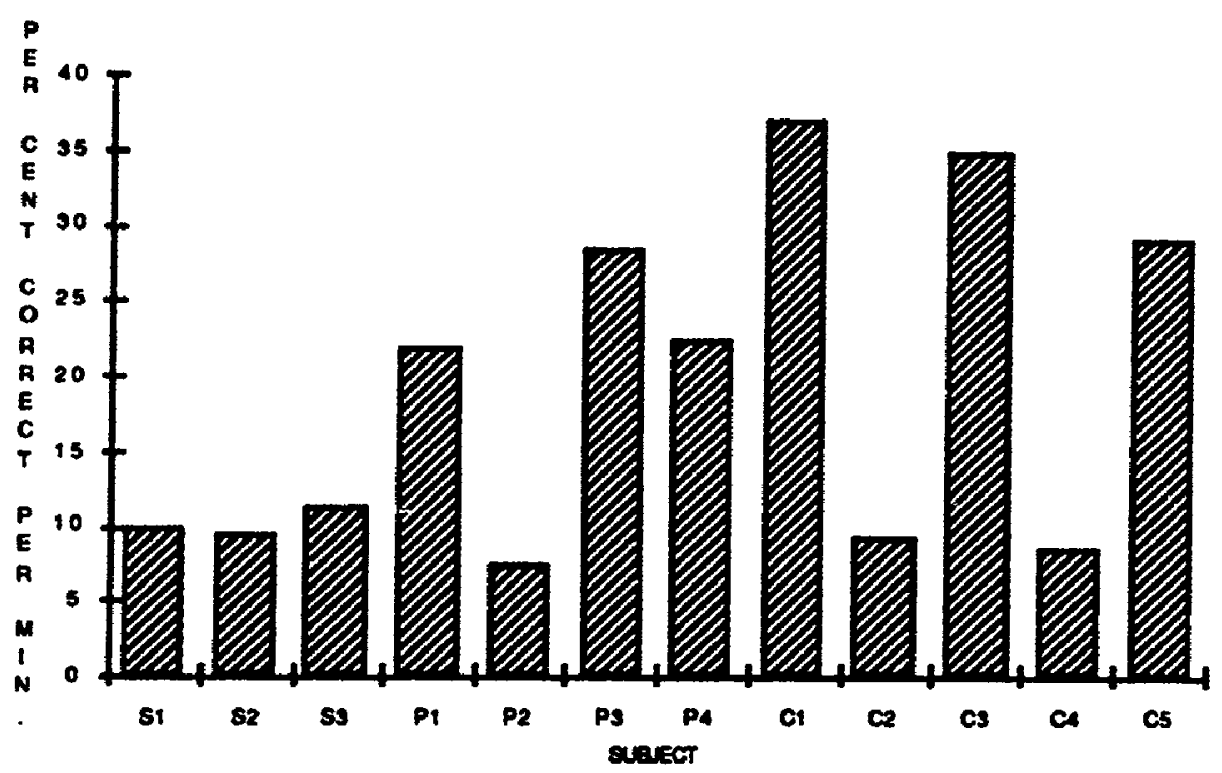

Eiqure A-3. The rate of recall (per cent of total aircraft attributes correctly recalled per minute). The average rate for non-controllers was $15.6 \%$ attributes/min. and for the controllers $23 . \overline{6}$ \%attributes/min. ("S" denotes student subjects, "P" simulator pilots, and " $\mathrm{C}$ " experienced controllers.)

\begin{tabular}{|l|l|}
\hline ATTRIBUTE & \multicolumn{1}{|c|}{ CRITERION } \\
\hline Identifier & $\begin{array}{l}\text { two or more letters/numbers } \\
\text { correct and in order }\end{array}$ \\
\hline $\begin{array}{l}\text { Present Position: } \\
\text { 2D location }\end{array}$ & within 5 nautical miles \\
3D location & within 5 nautical miles \\
\hline $\begin{array}{l}\text { Future Position: } \\
\text { 2D location }\end{array}$ & within 5 nautical miles \\
3D location & within 5 nautical miles \\
\hline Speed & plus or minus 50 Knots \\
\hline Heading & plus or minus 20 degrees \\
\hline
\end{tabular}

Table A-3. Criteria used for establishing ballpark accuracy. An altitude expansion factor of 12 was employed when calculating 3D accuracy. 52

52 It was found in the preliminary study that subjects typically expand the altitude scale when visualizing various altitude levels over a horizontal sector map. The expansion factor is linear and ranges from 9.7 to 18.5. Because subjects seem to mentally visualize the vertical dimension as much greater than it would be according to the corresponding horizontal scale, it was thought that this expansion should also be applied when calculating RMS accuracy of tecall. A moderate value of 12 was chosen. 


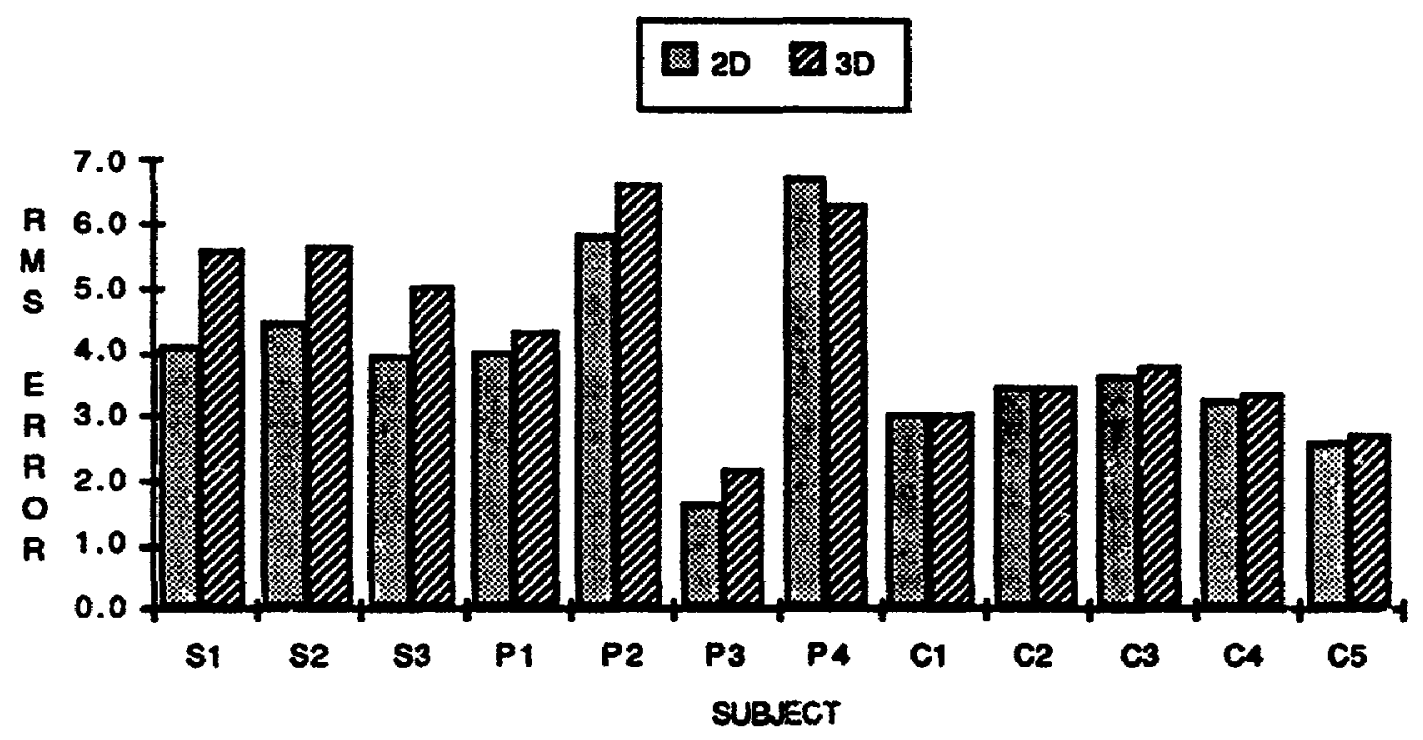

Figure.A4. Absolute accuracy of recall of aircraft two-and three-dimensional location. This measure does not take into account the number of aircraft recalled.

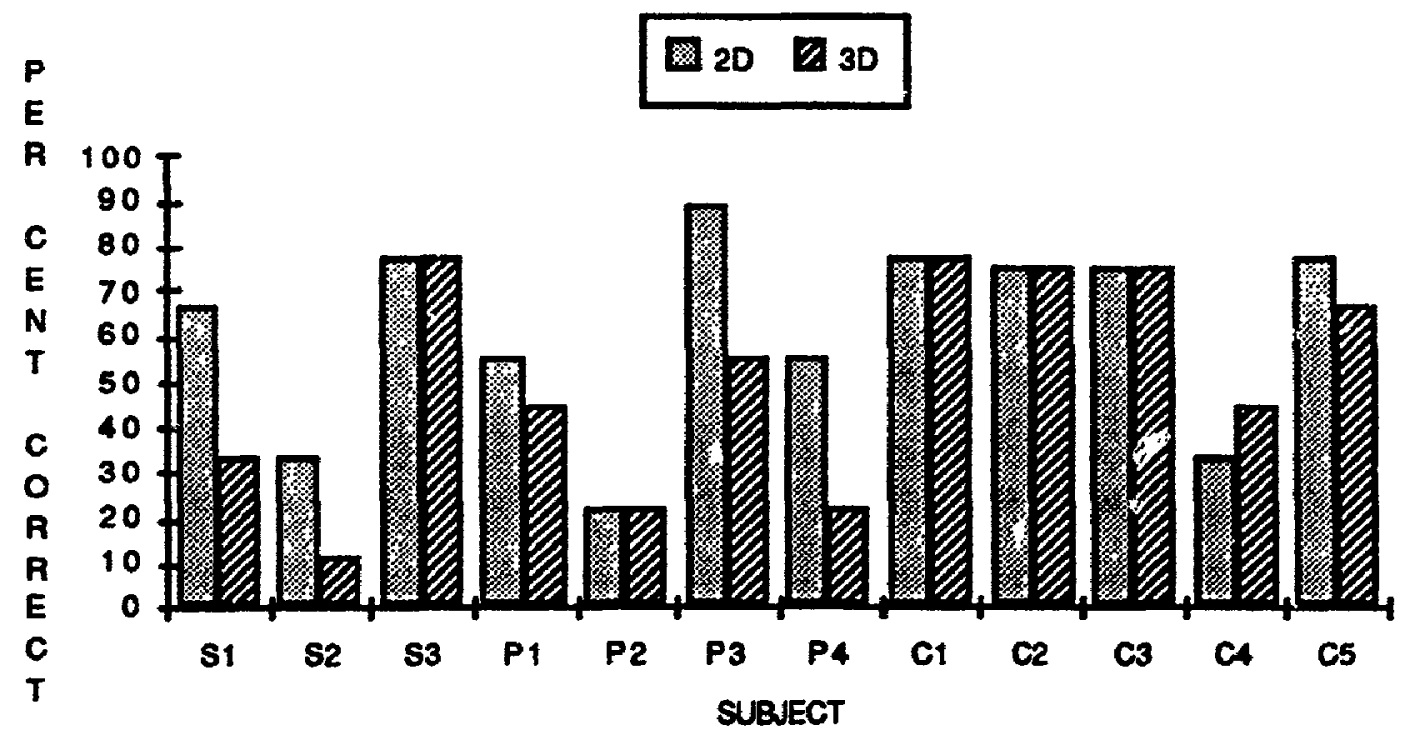

Figure A-5. Ballpark accuracy in recalling aircraft 2D location and 3D location (within 5 $\mathrm{nm}$ ) using RMS (root mean square) error calculation. (See Table A-3 for the criteria used to calculate ballpark accuracy.) 
two groups, significant t-tests were obtained only with respect to the three-dimensional results. 53 Bisseret (1970) and Moray (1980) also found that highly skilled controllers remember aircraft information better. Controller group performance was also more consistent (less variable) for absolute 2D accuracy and 3D accuracy. With C4 removed, it was also more consistent for ballpark 2D and 3D accuracy. .4

To sum up, three of the five controllers showed similar rapid recall rates and four had superior and more consistent accuracy for remembering three-dimensional location. 55 These results are especially impressive given that the controllers were on the average 29 years older than the students and 23 years older than the pilots. However, subjects S3 and P3 were also accurate with recall (Figure A-5). This result suggests that the ability to form a good representation of air traffic in short term memory may be aptitude-based rather than exclusively generated by training or experience. If good performance was restricted to the controller group only this premise would not be warranted.

It is not surprising that some non-controllers possess this ability. It could be assumed that those in the controller group were successful in ATC work based at least in part on the possession of good memory. Even controller C4's accuracy was notable, although his performance suffered in speed and quantity. These results suggest that recall efficiency (quantity and rate) and accuracy may be good measures of the aptitude for forming and using the picture.

Another interesting finding (see Table A-4) was that subjects remember certain aircraft attributes better than others. The data for the controller group were similar to those of Bisseret (1970) and Means, et al. (1988); their subjects also emphasized altitude and position information over other aircraft attributes.

53 Sce Appendix C-4.2 for t-test data.

54 Sce Appendix C-4.3.A for ANOVA data.

55 t-tests musl be viewed with caution with these data because of unequal group sizes and heterogeneity of variance (Howell, 1987). 


\begin{tabular}{|l|c|c|c|}
\cline { 2 - 4 } \multicolumn{1}{c|}{} & STUDENTS & PILOTS & CONTROLLERS \\
\hline 3D LOCATION & 41 & 36 & 68 \\
\hline 2D LOCATION & 59 & 56 & 68 \\
\hline HEADING & 52 & 50 & 54 \\
\hline IDENTIFIER & 26 & 58 & 34 \\
\hline SPEED & 37 & 11 & 18 \\
\hline
\end{tabular}

Table A-4. Aircraft attributes correculy recalled (ballpark accuracy, as a per cent correct of total available) in order of priority (based on controller responses). 3D location includes altitude. 
However, the students and pilots did not have the same priorities. ${ }^{56}$ The observation that simulator pilots stressed identifier fits with the kind of work they normally perform. When running the simulator they must respond to "radio" contacts from the controllers which begin with the call sign of the aircraft to be addressed. A two-way ANOVA of the recall accuracy data in Table A-4 (attributes by groups) indicated an effect for attribute only. 57

It is interesting to consicer the amount of information included in the recall task. For most subjects there were nine targets on the screen for this exercise, each with five attributes, making a total of $\mathbf{4 5}$ items to recall. That some subjects were able to recall something for all of these items and got up to 35 of these correct suggests that the limits of working memory were being exceeded and that chunking must have been occurring.

\section{A-3.3 Target Organization}

The expectation that controllers would have a unique and more refined system for grouping aircraft was not supported by the cluster analysis results. Most subjects organized the nine aircraft remaining on the TRACON monitor into five groups. Controllers were more variable than the non-controllers, employing from two to six groups. Upon review of the kinds of distinctions made between aircrafi, the controllers often employed the same rules as other subjects for forming clusters and in two cases used a very simplified structure, placing the aircraft into only two groups (arrivals and overflights). There was no indication that clusters of aircraft were recalled together, as found by Means, et al. (1988).

It was observed from inspection of the similarity comparison response sheets that the controller group tended to make extreme judgements close to or at the ends of the scales

\footnotetext{
56 A Kendall rank-order correlation between the three groups showed that students' order of recall correlated $T=0.20$ and pilots $T=-0.60$ with controller order of recall.

57 See Appendix C-4.3.B for ANOVA data.
} 
("Same" or "Different"). A count of these judgements was made for each subject. A judgement mark on a scale was considered exteme if it was $5 \mathrm{~mm}$ or less or $68 \mathrm{~mm}$ or more from the left end of the scale (total ieigth $73 \mathrm{~mm}$ ). There was a significant difference between the non-controller and controller groups. 58

In a study of air traffic controller decision making with regard to conflict detection Bisseret (1981) noted that trainee controllers were more accurate than experienced personnel when making estimates of the future separation of aincraft but considerably less cautious in predicting whether a conflict would occur. He also observed a difference in information processing :

the experienced controller might be making a 'judgement' based on a rough categorization of the problem, perhaps to the extent of simply acting or not; the trainees might try more to 'calculate' in order to obtain maximum knowledge of the problem before deciding to act or not. Thus, the trainees might seek a more thorough analysis of the problem. (p.91)

This decision-making style could explain why the controllers made more extreme judgements. They tend not to spend time calculating or analyzing but make an approximate decision based on the minimum requirements of the situation. The non-controllers appeared to expend more mental effort to make finer judgements. A number of the controller subjects emphasized that quick and decisive decision making is essential in air traffic control. The primary problem in ATC appears to be whether or not to act regarding aircraft separation and guidance. This, combined with time pressure, would rule out the luxury of extended deliberation.

\section{A-3.4 Interobject Distanse Judgements}

At the end of the recall simulation exercise, subjects were asked to a draw lines representing interaircraft separation. The actual $x, y$ and $x, y, z$ aircraft separations were calculated from their coordinates on the TRACON screen. Pearson product-moment

\footnotetext{
58 See Appendix $\mathbf{C}-\mathbf{4} .2$ for t-test data.
} 
correlations were computed between the set of lines drawn by each subject and the twoand three-dimensional separations of the targets.

As predicted earlier in the discussion of Pinker's (1980) experiments, the correlation between the actual two- and three-dimensional distances was high rendering this approach useless for correlation with subject perceptions. Therefore, the altitudes were multiplied by 12.6, the approximate altitude scale expansion being applied by subjects (this will be discussed in a subsequent section). It is not certain that this same scale expansion would apply to judgements made on the screen, but it was felt that the accuracy of the multiplier was not as important as using some significant scale expansion to roughly match subject perceptions. The correlations which resulted are shown in Figure A-6.

It was clear that most of the subjects were considering two-dimensional distance when making their judgments. The experimenter was careful not to use the words "distance" or "separation" when giving instructions so as not to imply that a certain type of judgement was required. It was hoped that subjects would use lateral or vertical separation or a combination of the two in their judgements depending upon how they conceived of the array of aircraft.

Four of the six subjects included the third (altitude) dimension in their estimates. Two of these were students and the others were controllers (instructors for Air Traffic Services). The only conclusion which can be reached at this time is that some subjects, including experienced controllers, include altitude in their spatial judgements but this type of perception is not a characteristic of any particular group.

\section{A-3.5 Subjective Altitude Judgements}

Before beginning the two simulation exercises which involved spatial model building and track prediction, subjects were asked to make two kinds of altitude estimates. The first was intended to determine the amount of altitude scale expansion each subject visualized when working with the a sector map. In addition, a target marker was produced and the 


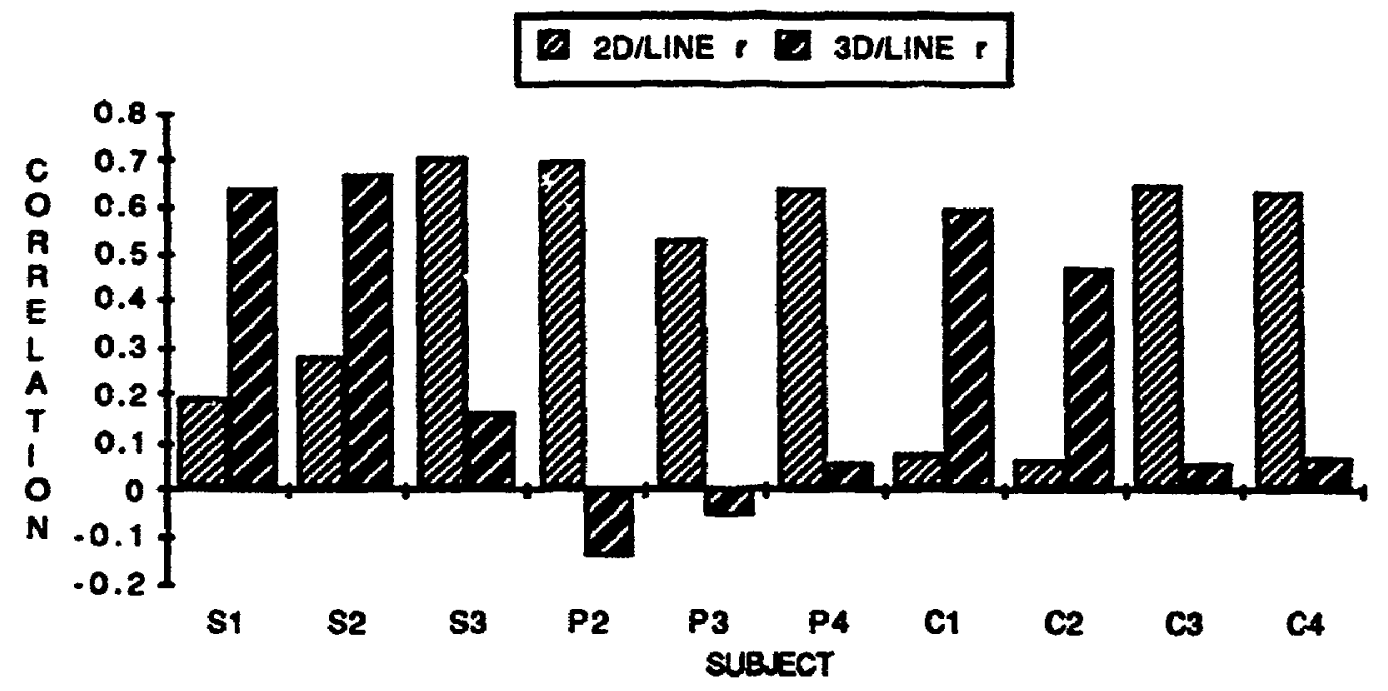

Eigure A-6. Pearson product-moment correlations of subject judgements of relative interobject distance with actual two- and three-dimensional interobject distances (altitude scaling factor applied). Data were unavailable for subjects P1 and C5. 
subjects set its slider to a series of different altitudes. This allowed a simple linear expression to be created which would check the accuracy of marker placements in later trials and also allowed the experimenter to determine if subjects were applying a linear scale in their slider altitude settings.

The average level of subjects' height of sector ceiling judgements $(20,000$ feet) was $354 \mathrm{~mm}$. This exercise was carried on from a seated position after the use of the TRACON simulation for about one hour. Judgements were not requested before the experiment began or at its completion. One subject commented that the level he chose was at his seated eye level; this would imply that his estimate would increase if he stood up. It is not known if other subjects were guided in this manner.

Given that the scale of the sector map being used in this exercise was $7.8 \mathrm{~mm}=1$ $\mathrm{nm}, 20,000$ feet would have been about $26 \mathrm{~mm}$. The above altitude estimates therefore reflected an expansion of this scale ranging from 9.7 to 18.5 times what would be expected. However, there were no significant differences in judgement between controllers and non-controllers. 59

This result is reminiscent of research by Thorndyke (1980) on the "filled distance effect". Thorndyke asked subjects to memorize simple schematic maps of roads and cities. He found that "when subjects were asked to estimate distances between target cities on the memorized maps (scanning their spatial image), those distances were systematically overestimated to the extent that more cities occurred between the target end points" (Wickens, 1984, p. 190). It may be that subjects exaggerate the altitude scale to the extent that they must conceive of aircraft operating at various levels within the airspace.

Subjects were then asked to position the slider on a plotting marker to four different altitudes $(5,000,10,000$ and $15,000 \mathrm{feet})$. The heights that the slider should be placed for

${ }^{59}$ Sec Appendix C-4.2 for t-test data. 
cach altitude if a simple linear expression was being used (altitude=K(height)) were calculated. It was found that subject judgements correlated at least $r=0.95$ with the linear predictions. Controllers were not more consistent in their slider placements. 60

\section{A-3.6 Head Turns}

Subjects were allowed to look at the TRACON screen as often as they wished when plotting the present and future positions of aircraft. The number of times the screen was consulted during the plotting tasks was recorded. It was thought that subjects with a more persistent picture or mental model might need to consult the screen less often. However, given that the air traffic was not moving during the plotting trials, this can only he taken as a rough estimate of resiliency.

One subject (P4) made very few head turns toward the display of aircraft on the TRACON monitor. In fact, the screen was only consulted an average of once during each plotting trial. This was unusual in the context of other subjects' performance and, if accuracy in plotting target position is any indicator (see Figures A-4 and A-5), head-onneck movements may have been reduced at the expense of performance (not much time was saved, as seen in Figure A-3). With this subject's data removed a t-test revealed that controllers made significantly fewer head turns perhaps indicating a more resilient picture. ${ }^{61}$ Averaged across all plotting tasks, controllers checked the TRACON screen about every 9 seconds, pilots every 10 seconds (excluding P4), and students every 6 seconds.

\section{A-3.7 Response Time}

As mentioned in the rationale, it was hoped that the time required to complete the plotting of aircraft position in three dimensions would provide some indication of a

60 See Appendix C-4.2 for t-test data.

${ }^{61}$ See Appendix $\mathrm{C}-4.2$ for t-test data. 
subject's skill with spatial reasoning and projection. However, there was no significant difference between controllers and non-controllers. 62

\section{A-3.8 Model Building: All Trials}

The plotting tasks were divided into two main groups based on type of exercise. The first thing subjects were asked to do was to plot the present positions of the aircraft on the TRACON screen. This "model building" was considered to be a test of how well they could reproduce three-dimensional locations of targets from a two-dimensional image. The altitude error in the 3D plots was based on the height at which a subject placed the slider (converted into feet by using the formula derived from their earlier practice trials) rather than the altitude the subject wrote on the sector map.

Both the two- and three-dimensional RMS error calculations indicated a subject's ability to build a three-dimensional model in the absence of much scale information. Neither the sector map nor the markers had any visible metric against which to judge horizontal or vertical distance (except for their boundaries and, in the case of the map, airway and runway markings).

In general, subjects were able to place the $x, y$ location of targets on the sector map within one to two miles (representing an etror of about 8 to $16 \mathrm{~mm}$ ). As with response time, there was a pattern of slightly more consistent, accurate two-dimensional ability within the controller group, but this did not reach significance. ${ }^{63}$ Three-dime. sional accuracy was also unevenly distributed and it is notable that the controller subjects were generally equal to or worse than the other subjects when altitude was taken into account. ${ }^{\text {id }}$ These findings did not support the hypothesis that controllers have better developed spatial abilities than non-controllers, at least as operationalized in this experiment.

62 Sec Appendix C-4.2 for t-test data.

${ }^{63}$ See Appendix C-4.2 for t-test data.

64 Sec Appendix C-4.2 for t-test data. 


\section{A-3.9 Prediction: All Trials}

Prediction of future position was considered to be a different exercise from building a spatial model of the targets visible on the TRACON screen. It was necessary to extrapolate from what was visible on the screen to a predicted future state.

Unfortunately, accuracy measurements were confounded to some unknown degree with model building ability. If a subject showed a certain amount of "modeling error" in merely transposing what was visible on the TRACON screen to the plotting board, it could be assumed that a similar degree of error was present when the predictive plotting tasks were performed. Therefore, these errors were probably a combination of model building inaccuracy and mistakes made in projecting target future position.

In an attempt to factor out one source of error, the results in this section were compared to those in model building. If a subject obtained about the same degree of accuracy when making a prediction as when representing present position, it could be assumed that predictive accuracy was very good. The degree to which there was a difference, however, should have given a measure of projective error.

\section{A-3.9.1 Two Minute}

There was no significant difference between controllers and non-controllers for accuracy in making predictions of aircraft position two minutes ahead (collapsed over arrivals and overflights). 65 However, the ability to model build had to be factored out of predictive accuracy in order to obtain a useful sense of how well a subject could project future position.

Accordingly, two- and three-dimensional model-building errors were subtracted from the two minute prediction data. Although it appeared from inspection of the results that controllers were somewhat less accurate as compared to the other subjects when asked to

${ }^{65}$ See Appendix C 4.2 for t-test data. 
project two-minute position, there were no convincing group differences. ${ }^{66}$ Two- and three-dimensional predictive ability was not a strength of a particular subject group, but was skill certain subjects seemed to possess. For example, in considering accuracy for plotting present and two minute positions, subjects S1, S2, and P2 showed consistently good two- and three-dimensional accuracy.

\section{A-3.9.2 Five Minute}

Prediction of five minute position was restricted to the two trials concerning arrivals. As in the case with two minute projections, the difference between model and five minute error was used as an indicator of predirtive ability. In general, there was more agreement between two- and three-dimensional accuracy for predicting the five minute position of approaching aircraft than in the two minute trials. However, although it appeared that the controllers were somewhat more accurate and consistent than the non-controllers, there were no significant differences between groups in either the overall error results or when model error was removed. 67

\section{A-3 9.3 Present Position and 2 Minute Prediction: Arrivals versus Overnights}

The preceding sections reviewed the results of the plotting tasks for present and future position collapsed across condition (arriving versus overflying). However, the task of predicting the positions of arriving and overflying aircraft would draw upon different skills and these data were analyzed separately.

The prediction of future positions of arrivals required forming a plan and following it. However, the future $x, y$ positions of overflights should have been easier to predict because they were on predetermined, straight-line routes. The differences between twoand three-dimensional errors for arrivals and overflights were calculated. Thus if a subject

${ }^{66}$ See Appendix $\mathrm{C}-4.2$ for t-test data.

67 See Appendix C 4.2 for t-test data. 
achieved a negative score, it was an indication of superior accuracy in plotting the present or future positions of overflying aircraft as opposed to arrivals. A positive score suggested the opposite. Model building error was not factored out of these results and so the error measurements should be considered as only a rough indication of predictive ability.

There was no pattern in the present position two-dimensional error differences between controllers and non-controllers but controllers were better with overflying aircraft when altitude was included. 68 This might indicate better spatial abilities but it should be noted that the exercise involving overflying aircraft was later in the experiment than the one involving arrivals; there may have been an improvement in skill.

When prediction of position and altitude two minutes ahead was involved, there was evidence of contrasting abilities on the basis of air traffic control experience, as seen in Figure A-7. Five of the students and pilots (non-controllers) had about equal accuracy or were better at predicting overflying aircraft as opposed to arrivals. However, three of the controllers were equal or somewhat more accurate with arrivals (except for subjects $\mathbf{C} 4$ and C5).

However, in spite of the appearance of Figure A-7, there were no significant differences between controllers and non-controllers. ${ }^{69}$ However, when controller C2's disproportionately large 2D error was removed as an outlier and a significant difference in 2D accuracy emerged. Controllers were better with arriving aircraft and non-controllers were more accurate with overflying aircraft. ${ }^{70}$

Moray (1980) reported research which supports these data. When controllers have an aircraft under supervision its future position is more predictable than when the aircraft is under a pilot's control; in the latter case there is more uncertainty as to what

68 See Appendix $C 4.2$ for t-test data. 69 See Appendix C 4.2 for t-test data. 70 See Appendix C-4.2 for t-test data. 


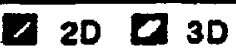

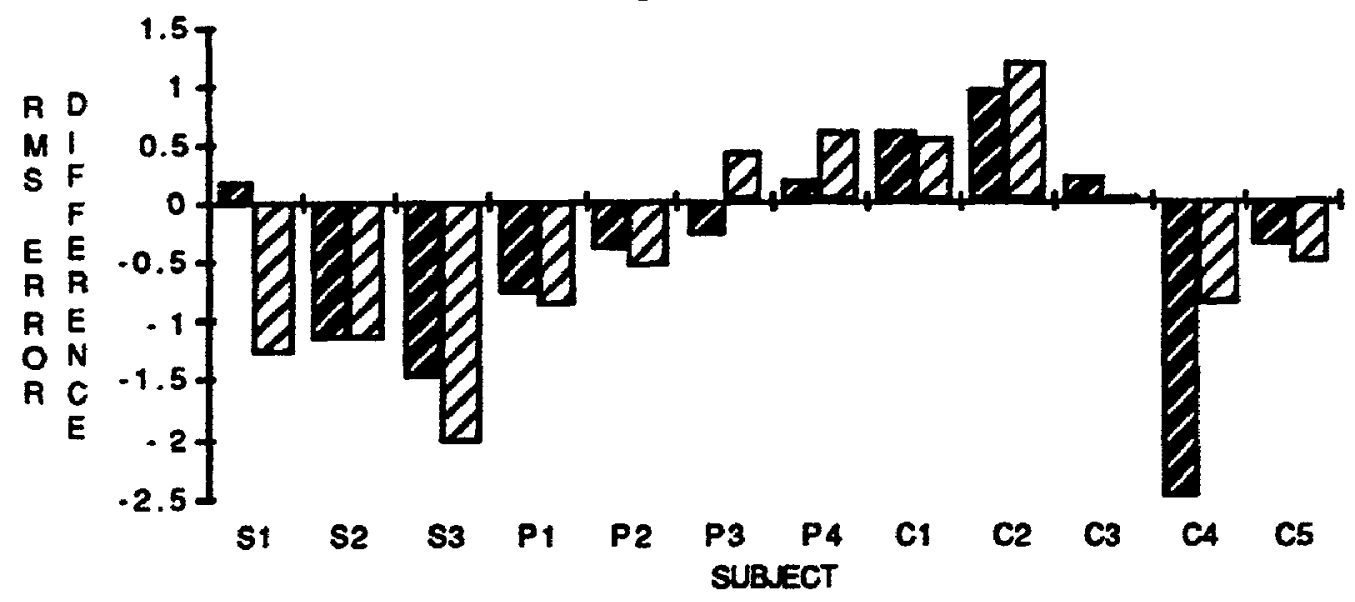

Fiqure A-7. The differences in RMS error for plotting the 2 minute positions of arriving versus overflying aincraft. The error for arriving aircraft was subtracted from that for overflying aircraft. Therefore, negative differences indicate better accuracy for plotting overflying targets and positive differences suggest better accuracy for arriving aircrafi. 
the pilot will do. This could make the monitoring of overflights subjectively more difficult than the control of arrivals.

\section{A-3.9.4 Present Position and 2 Minute Prediction: Conflicts versus Non-Conflicts}

As discussed in the rationale, it was predicted that if subjects (especially controllers) used spatial imagery when controlling aircraft this ability might only be employed when potential conflict situations were being conceptualized. If this assumption was correct, it would be expected that controllers would show lower errors when predicting the positions of conflicting (as compared to non-conflicting) aircraft. As in the previous section, differences in error for plotting the present positions of conflicting and non-conflicting overflying aircraft were calculated. The data showed that over half of the subjects had about the same accuracy in both situations. However, four of the five controllers seemed somewhat more accurate when modelling the present position of non-conflicting aircraft. However, tests of between group differences did not reach significance. ${ }^{71}$

With regard to two minute predictions, subjects' errors went in either direction, with three of the five controllers seeming to find non-conflicts slightly easier to predict (although the error differences were only about 0.5 miles). Contrasts of the controller and noncontroller groups did not reach significance. 72 It may be that subjects employed their spatial abilities to an equal extent for all plotting tasks.

\section{A-3.10 Fault Detection}

Various kinds of pilot errors were intentionally injected into the simulation exercises to alter the altitude or heading of overflying or approaching aircraft. Figure A-8 is plot of the per cent of errors detected and average response time to discover the error. Good

71 See Appendix C-4.2 for t-test data.

72 See Appendix C-4.2 for t-test data. 


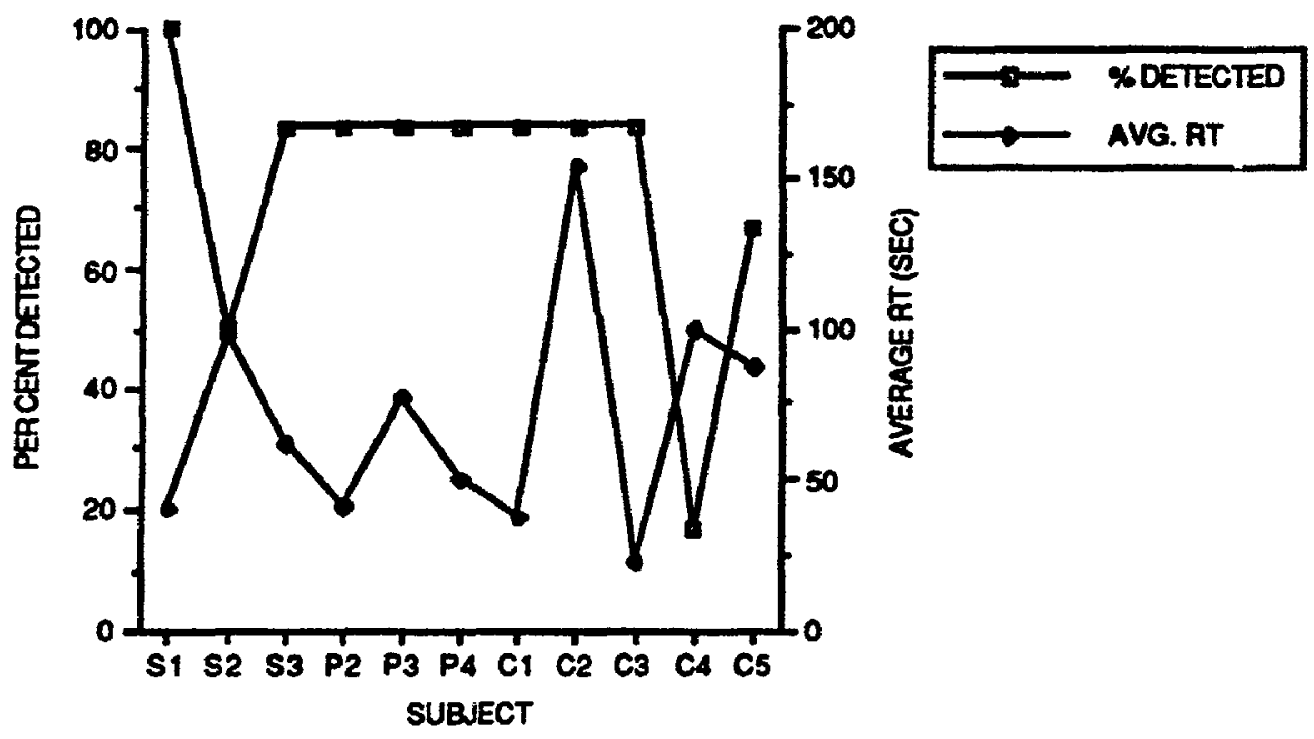

Eiqure A-8. The number of pilot errors detected of any type and the average response time to detect them. Good performance was a combination of high detection rate and fast response time. 
performance in cletecting faults included a high rate of detection and rapid response. With this in mind, subjects S1, S3, P2, P4, Cl and C3 showed good abilities.

It is interesting that five of the six vigilant subjects also did well on the recall task described earlier. Considering that error detection probably relies on the visual sampling of targets on a regular basis and the comparison of the perceived attributes with those in memory (or in the picture), it is not surprising that those with good recall ability also detect faults well. A Kendall rank-order correlation of the fault detection data with accuracy of $2 D$ and 3D absolute and ballpark recall acsuracy yielded significant correlations of all recall measures with per cent of faults detected but not with response time (see Table A-4).

It may be that the subjects who had good a picture but who performed poorly on fault detection were not making the necessary comparisons for some reason. Perhaps their attention was completely captured by the primary task of controliing aircraft in an unfamiliar airspace using a novel radar display.

\section{A-4. Conclusions}

Table A-5 is a restatement of the hypotheses found in Section A-1.5. The aim was to determine if controllers differed from non-controllers on a number of measures related to the recall, organization, and spatial aspects of the picture. As can be seen, the predictions regarding recall accuracy, prioritization of recall of aircraft attribute, resiliency of the picture, and accuracy in prediction of arriving versus overflying aircraft were supponed. Notably, most of the hypotheses regarding superior spatial skills on the part of controllers were rejected. Please see Section 2.3 for more detailed conclusions of the Preliminary Study. 


\begin{tabular}{|c|c|c|c|}
\cline { 2 - 4 } \multicolumn{1}{c|}{} & Per Cent Detected & Response Time & $\begin{array}{c}\text { Rale } \\
\text { (\% Detecied/RT) }\end{array}$ \\
\hline Absolute 2D & 0.53 & -0.09 & 0.13 \\
\hline Absolute 3D & 0.53 & -0.13 & -0.16 \\
\hline Ballpark 2D & 0.56 & 0.02 & 0.20 \\
\hline Ballpark 3D & 0.53 & -0.05 & 0.20 \\
\hline
\end{tabular}

Table A-4. Kendall rank-order correlations of recall accuracy (left column) with error detection measures (top row). T-values of over 0.45 are significant at p<.05.

\begin{tabular}{|l|c|c|}
\hline \multicolumn{1}{|c|}{ HYPOTHESIS } & SUPPORTED & REJECTED \\
\hline 1. Task-related priority of recall of aircraft. & & \\
\hline $\begin{array}{l}\text { 2. Better recall performance in terms of rate, amount, and } \\
\text { axcuracy. }\end{array}$ & & \\
\hline $\begin{array}{l}\text { 3. Prioritization of recall of aircraft atributes in line with } \\
\text { previous findings (Bisseret, 1970; Means et al., 1988). }\end{array}$ & \\
\hline $\begin{array}{l}\text { 4. A common, more refined, and task-related basis for the } \\
\text { clustering of targets into groups. }\end{array}$ & \\
\hline $\begin{array}{l}\text { 5. Correlation of interobject distance judgments with a three- } \\
\text { dimensional spatial image. }\end{array}$ & \\
\hline $\begin{array}{l}\text { 6. Higher, more consistent, and more uniform subjective } \\
\text { ceiling judgments. }\end{array}$ & \\
\hline $\begin{array}{l}\text { 7. Fewer head turns to check the TRACON screen during } \\
\text { plotting tasks. }\end{array}$ & \\
\hline \begin{tabular}{l} 
8. Better response time and accuracy with plotting tasks. \\
\hline 9. More accuracy in predicting arrivals.
\end{tabular} & \\
\hline $\begin{array}{l}\text { 10. More accuracy in predicting conflicting (as opposed to non- } \\
\text { conflicting) aircraft positions. }\end{array}$ & \\
\hline 11. Better fault detection. & \\
\hline
\end{tabular}

Table A-5. Support or rejection of hypotheses stated for the Preliminary Study. It was predicted that controllers would be superior or significantly different on all measures. 


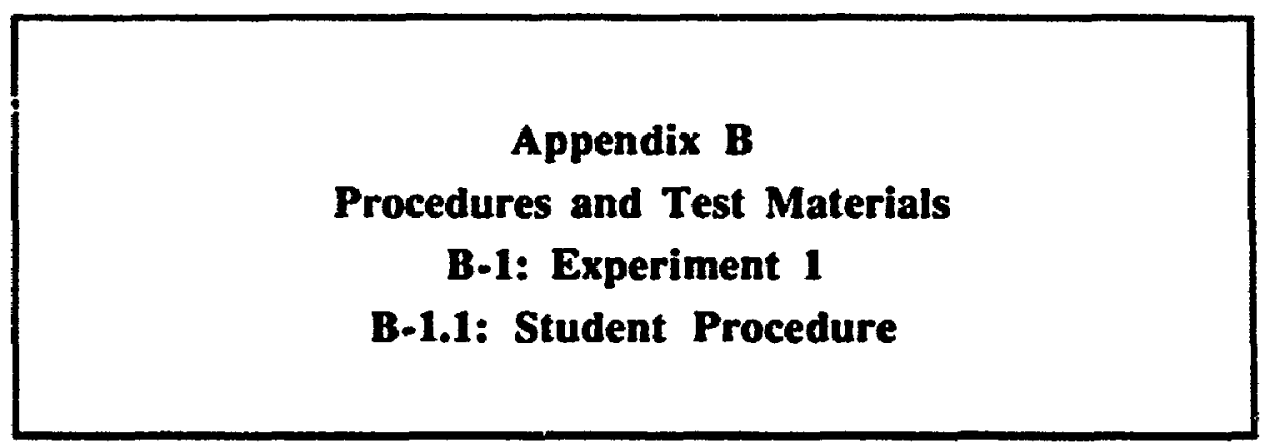


1. Give each $\$$ a clipboard with an example map (loose), a blank sector map, and a pencil.

2. Say to the Ss:

During the simulation exercise you are about to begin, we will stop and ask those of you who are acting as controllers to remember all of the aircraft you are working with. You who are data people do not have to do anything, but I want you to turn away from the flight data strips while the person on radar is doing the recall task.

Proceed with the exercise as you normally would. At a certain time, I will stop the simulation and tell you to start. The instructor will dim your radar sereen and you should immediately take the pencil provided and start marking the positions of all of the currently active aircraft at the moment the exercise was stopped. You will be timed, so try to start as soon as you can.

I will now describe how to record aircraft information. Look at the box in the upper left corner of your "Example Map". Write your name, workstation number, and the date on this map.

Each time you record an aircraft's position on the map, make a dot and then note its identifier, altitude, and speed. Draw a line or write a compass bearing giving its heading.

Be as accurate and as fast as possible. Please do not look at anything on your workstation while you are trying to remember of this information.

As soon as you have finished writing down all of the aircraft you can remember, say "done" so that the instructor who will be timing you will know you are finished. After you have signalled that you are finished, you should not write down any further aircraft.

3. Practice: Say to the Ss:

Now, on the example map, I would like you all to practice writing down the information I have requested about two aircraft. The first example aircraft is:

$\begin{array}{llllll}\text { IDENT. } & \text { LOCATION } & & \text { ALT. } & \text { SPD. } & \text { HDG } \\ \text { 1.CFCO56 } & \text { YSI/262 } & \text { 70DME } & 25,600 & 450 & 085 \\ \text { 2.GVVB } & \text { YAC295 } & \text { 50DME } & 28,000 & 450 & 295\end{array}$

1. "Stillpond 262 radial at $70 \mathrm{DME}$

2. "Avalanche 295 radial at $50 \mathrm{DME}$ "

4. Take the example maps away from the Ss, leaving the blank map on the clipboard. Say:

Please write your name, workstation number, and the date on the map.

We will now go ahead with the simulation exercise. Remember, when I say "start the recall task", immediately turn away from your radar and start recording all of the aircraft you can remember in the way we have just discussed. There is an example of how the 
information should be recorded in the upper left-hand corner of each map you will be using.

Work as quickly and accurately as possible with the recording of aircraft information. Do not forget to say "done" to the instructor at the moment whis you have finished this remembering all of the aircraft you can.

Data people, please turn your chairs around so that you are looking away from the flight data strips until we restart the exercise.

Are there any questions before we get started? 
Appendix B-1.2

Instructor Procedure 
During the experimental exercise, the simulation will be interrupted and the student you are working with will be asked to write down all the aircraft they can remember on a blarik sector map.

When I say "start the recall task", you should turn the screen brightness down and the student should immediately tum away from the radar and begin marking aircraft information down on his/her map as quickly and as accurately as possible. As soon as the student has put their hands on the map, please start your stopwatch.

Continue timing until the student says "done" and then immediately stop your watch and write down the time below. After the student says "done" he/she should not write down any further information.

Please note below any problems which occurred, such as if the student delays in turning away from the radar, attempts to note down more aircraft after saying "done", if you p:oblems with timing, etc. If the student wrote down any further information after you stopped timing, please take a minute to circle the extra items on each map.

Student's Name (or number):

Your Name:

Date of Trial:

Stopwatch Time:

Problems: 
Appendix B-1.3

Sector Map 


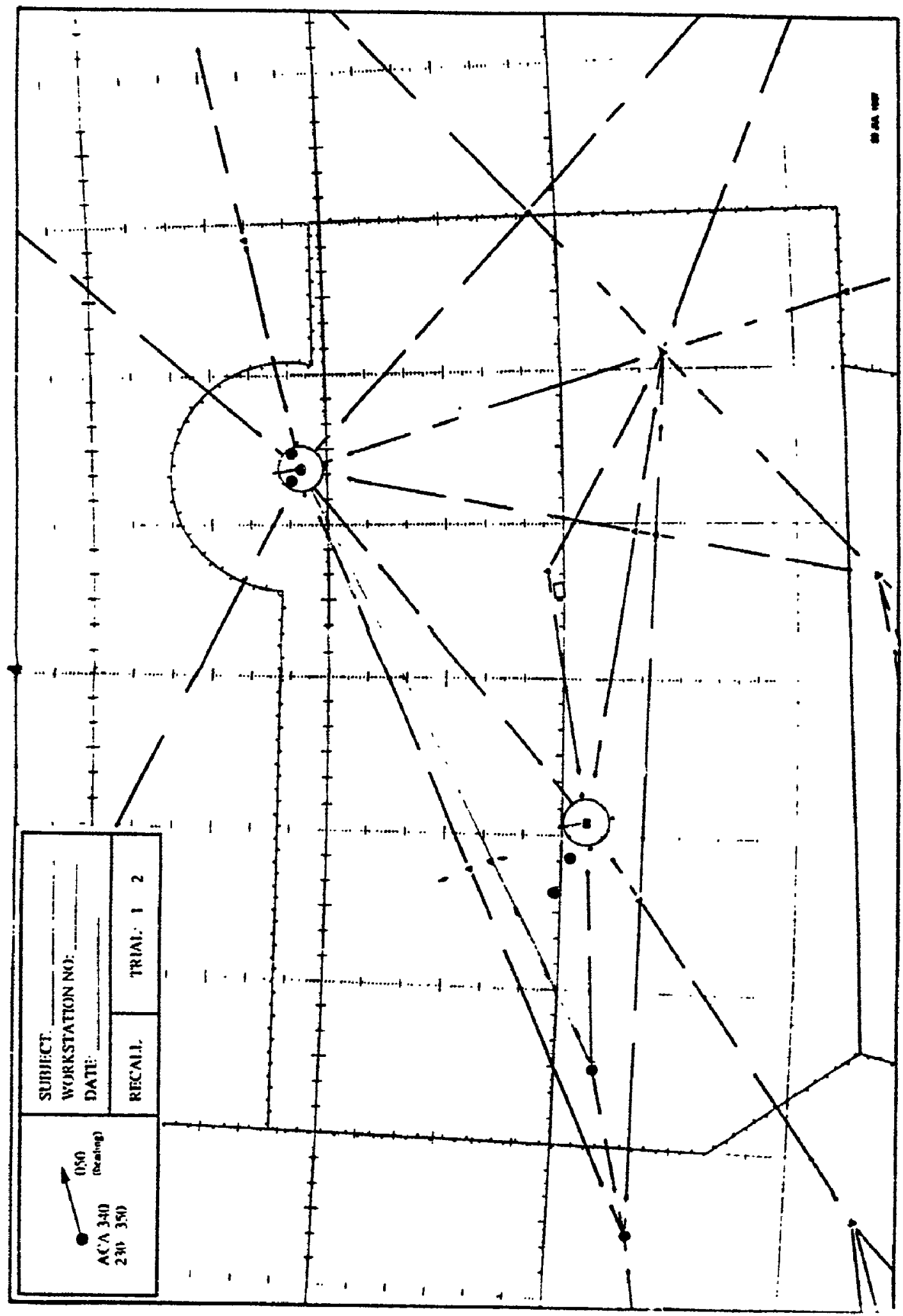




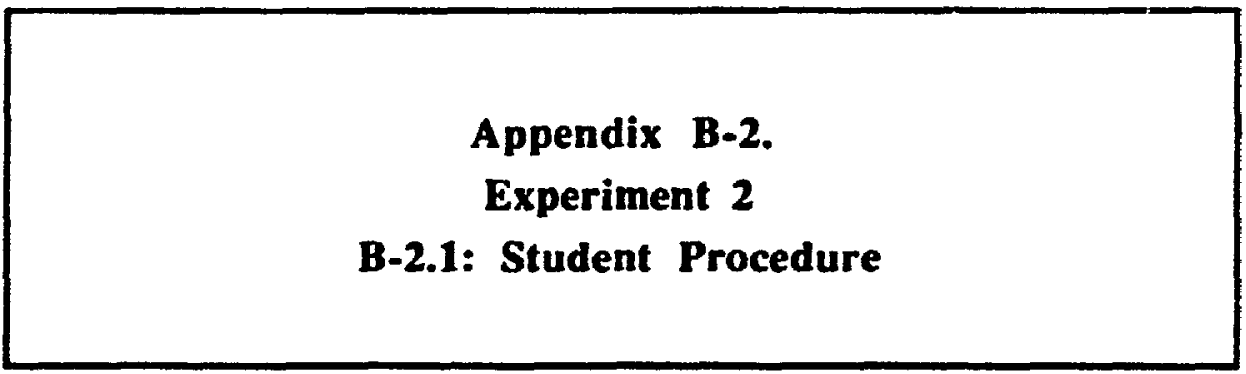


Say to the Ss:

At the end of the upcoming simulation exercise you are about to begin, we will stop and ask you to remember all of the aircraft you are working with at that moment in time. Proceed with the exercise as you normally would. At a certain time, your instructor will ask you to start. Please then turn away from your flight strips and immediately take the pencil provided and start marking the information requested on all of the currently active aircraft at the moment the exercise was stopped. You will be timed, so try to stan as soon as you can.

I will now describe how to record aircraft information. Look at the box in the upper left comer of your map. Write your name, position number, and the date on this map.

Each time you record an aircraft's position on the map, make a dot and then note its identifier, altitude, and speed. Please use the notation shown on the map. Draw a line or write a compass bearing giving its heading or the radial it is on.

$\mathrm{Be}$ as accurate and as fast as possible. Please do not look at anything on your flight strips while you are trying to remember of this information.

As soon as you have finished writing down all of the aircraft you can remember, say "done" so that the instructor who will be timing you will know you are finished. After you have signalled that you are finished, you should not write down any further aircraft.

We will now go ahead with the simulation exercise. Remember, when I say "start the recall task", immediately turn away from your strips and start recording all of the aircraft you can remember in the way we have just discussed. There is an example of how the information should be recorded in the upper left-hand corner of each map you will be using.

Are there any questions before we get started? 
Appendix B-2.2

Instructor Procedure 
At the end of each experimental exercise, please ask the student you are working with to write down all the aircraft they can remember on the sector map.

When you ha'r decided the simulation is over, immediately tell the student to begin marking aircr $\mathrm{ft}$ information down on his/her map as quickly and as accurately as possible. As soon as the siudent has put their hands on the map and is ready to go, please start your stopwatch. The student should be asked to turn away from their flight strips.

Continue timing until the student says "done" and then immediately stop your watch and write down the time in the space below. After the student says "done" he/she should not write down any further information. Also write down the actual clock time the simulation ended.

While the student is working, mark down on your map the actual aircraft information as accurately as possible following the same format the students are using (as shown on the upper left hand corner of your map). However, for each aircraft please also add the time from the flight strip that the aircraft passed by its last fix.

Please note below any problems which occurred, such as if the student delays in turning away from the strips, attempts to write down more aircraft after saying "done", if you problems with timing, etc. If the student put down any further information after you stopped timing, please take a minute to circle the extra items on his or her map.

Student's Name:

Your Name:

Stopwatch Time:

Simulation Clock Time:

Problems: 


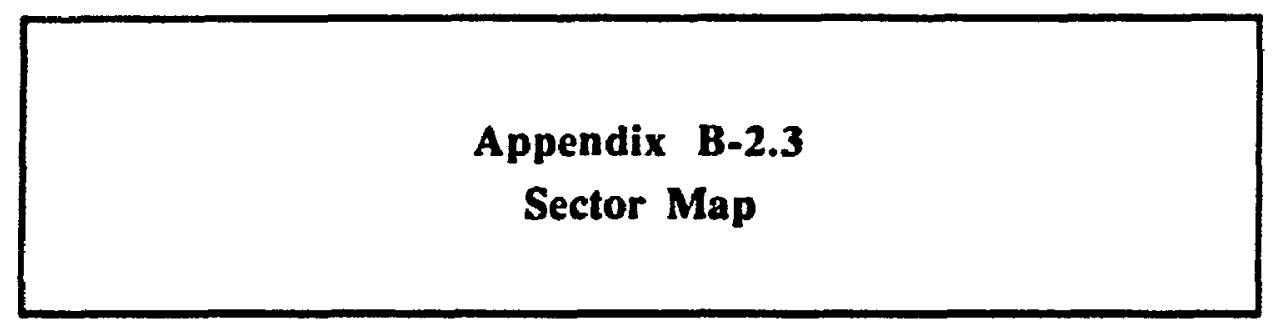


159

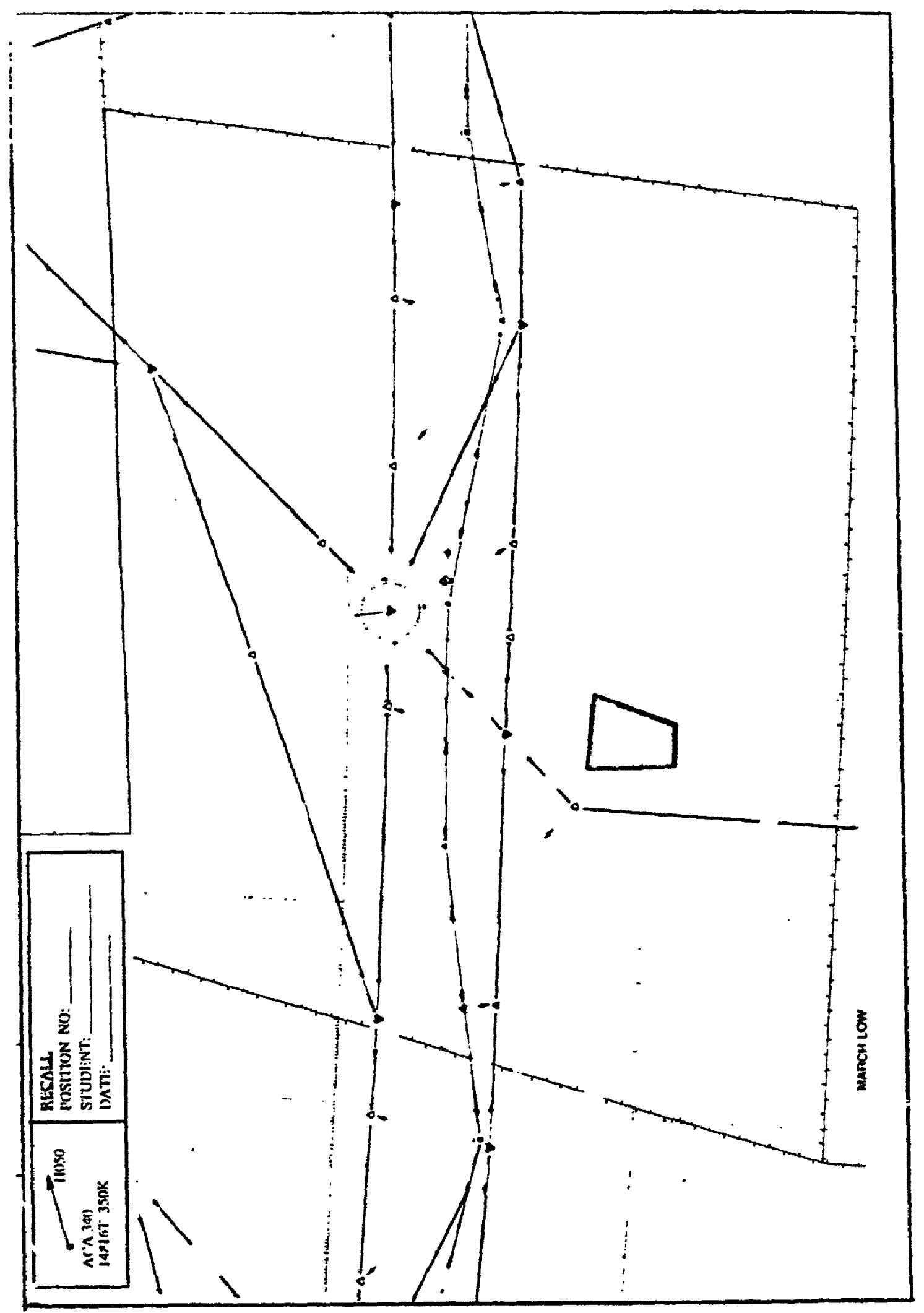


Appendix B-3.

Experiments $1 \& 2$ Tests and Criterion Measures

B-3.1: Differential Aptitude Test 
DO NOT MAKE ANY MARKS in THIS BOOKLET $\cdots$

\section{VERBAL REASONING}

\author{
DIRECTIONS
}

Marix roün àniméne

: ON THE BRPARATE :d

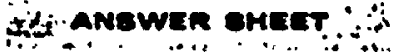

Find the space for Verbal Reasoning on the Answer Sheet.

Each of the fifty sentences in this test has the first word and the last word left out. You are to pick out words that will fill the blanks so that the sentence will be true and sensible.

For each sentence you are to choose from among five pairs of words to fill the blanks. The first word of the pair you choose goes in the blank space at the beginning of the sentence; the second word of the pair goes in the blank at the end of the sentence. When you have picked the pair to fill in the blanks, mark the letter of that pair on the Answer Sheet, after the number of the sentence you are working on. Here are some examples:

Example X. is to water as eat is to ......

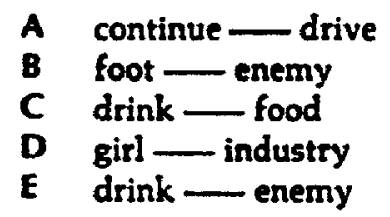

Drink is to water as eat is to food. Drink is the first word of pair $C$ and food is the second word of pair $C$, so the circle for $\mathbf{C}$ has been filled in on line $X$ of your Answer Sheet.

Now look at the next example.

Example Y. is to night as breakfast is to ......

$$
\begin{array}{ll}
\text { A supper - corner } \\
\text { B gentle - morning } \\
\text { C door - corner } \\
\text { D flow - enjoy } \\
\text { E supper - morning }
\end{array}
$$

Supper is to night as breakfast is to morning. Pair $E$ has both supper and morning; supper fits in the blank at the beginning of the sentence and morning fits in the blank at the end. On your Answer Sheet, the circle for $E$ has been blackened on line $Y$ to show that pair $E$ is the right one.

Now do the next example yourself. Blacken the correct space on line $\mathbf{Z}$ of your Answer Sheet.

Example Z.

.... is to one as second
A troo - middle
B first - fire
C queen - hill
D first - two
E rain - fire

First is to one as second is to two. First fits in the blank at the beginning of the sentence, and two belongs in the blank at the end. First and two make up pair $D$, so you should have filled in the circle for $D$ on line $Z$ of your Answer Sheet.

Fill in only one answer space for each sentence.

You will have $\mathbf{3 0}$ minutes for this test. Work as rapidly and as accurately as you can. If you are not sure of an answer, mark the choice that is your best guess. 
DO MOT MAKE ANY

MARKS IN THIS

BOOKLET

\section{NUMERICAL ABILITYY}

\section{DIRECTIONS}

Mank roun answens

ON THE BEPARATE ANBWEA BHEET

Find the space for Numerical Ability on the Answer Shee:-

This test consists of forty numerical problems. Next to each problem there are five answers. You are to pick out the correct answer and mark its letter on the Answer Sheet. If you do not find a correct answer among the first four choices, blacken the circle for $\mathrm{N}$ as your answer. Choice $\mathrm{N}$ for every problem is none of these, which means that a correct answer is not among the first four choices. Only one answer should be marked for each problem. Do your figuring on the scratch paper you have been given, and reduce fractions to lowest terms. Here are some examples:

$$
\begin{aligned}
& \text { Example X. Add } \\
& \begin{array}{lll} 
& \text { A } & 14 \\
13 & \mathbf{E} & 16 \\
12 & C & 25 \\
& D & 59 \\
& N & \text { none of these }
\end{array} \\
& \begin{array}{lll}
30 & \text { A } & 8 \\
20 & \text { C } & 15 \\
2 & \text { C } & 16 \\
& \text { D } & 26 \\
& N & \text { none of these }
\end{array}
\end{aligned}
$$

Remember-Each answer must be reduced to its simplest terms. For example, if two choices are $1 \frac{1}{2}$ and $1 \%$, only the $1 \frac{1}{2}$ is correct.

DO ALL YOUR FIGURING ON THE SEPARATE SHEET OF SCRATCH PAPER.

You will have 30 minutes for this test. Work as rapidly and as accurately as you can. Do not spend a long time on any one problem. If you are not sure of an answer, mark the choice that is your best guess.

DO NOT TURN THE PAGE UNTIL YOU ARE TOLD TO DO SO. 
$\therefore$ Do not MAKe äry

$\because$ MARKe IN TMIS

$\therefore$. EOOKLET . . "

\section{ABSTRACT REASONING}

\section{DIRECTIONS}

Mank youn anewere

ON THE oRpanate

ANBWER BMEET

Find the space for Abstract Reasoning on the Answer Sheet.

In this test you will see rows of designs or figures like those below. Each row across the page is one problem. You are to mark your answers on the Answer Sheet.

Each row consists of four figures called Problem Figures and five called Answer Figures. The four Problem Figures make a series. You are to find out which one of the Answer Figures would be the next (or the fifth one) in the series of Problem Figures. Here are two examples:
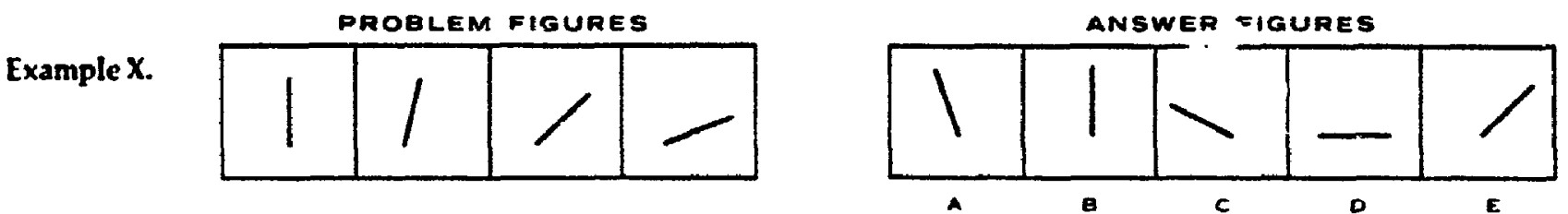

In Example X, note that the lines in the Problem Figures are falling down. In the first square the line stands straight up, and as you go from square to square the line falls more and more to the right. In the fifth square the line would be lying flat, so the correct answer-chosen from among the Answer Figures-is $D$. Therefore, the circle for $D$ has been filled in on line $X$ of your Answer Sheet.
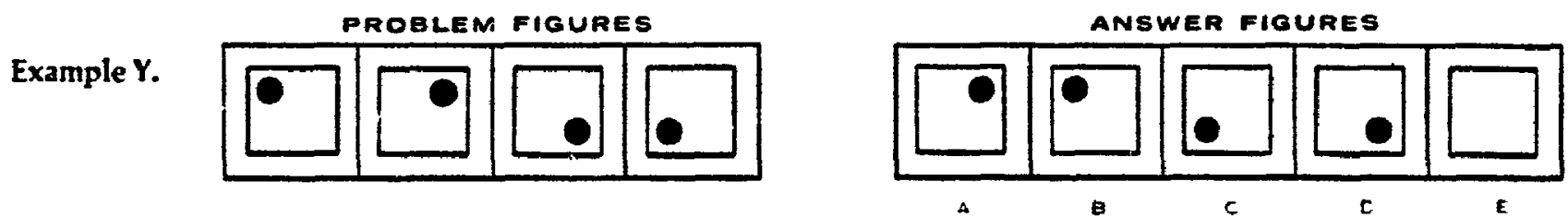

In Example $Y$, study the position of the black dot in the Problem Figures. Note that it keeps moving around the square clockwise: upper left corner, upper right corner, lower right comer, lower left corner. In what position will it be seen next? It will come back to the upper left corner. Therefore, B is the correct answer. The circle for B has been filled in on line $Y$ of your Answer Sheet.

Remember-You are to select, from among the Answer Figures, the one figure that belongs next in the series.

You will have $\mathbf{2 0}$ minuter for this test. Work as rapidly and as accurately as you can. If you are not sure of an answer, mark the choice that is your best guess.

DO NOT TURN THE PAGE UNTIL YOU ARE TOLD TO DO SO. 
DS DO NOT MAKE ANY F. MARKS IN THIS BOKLET

\section{CLERICAL SPEED AND ACCURACY}

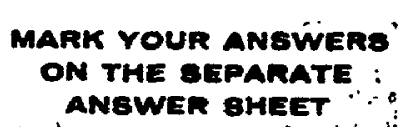

ANBWER BHEET

\section{DIRECTIONS}

Find the space on the Answer Sheet for Part I of Clerical Speed and Accuracy.

This is a test to see how quickly and accurately you can compare letter and number combinations. On the following pages are groups of these comkinations; each test item contains five. These same combinations appear after the number for each test item on the Answer Sheet, but they are in a different order. You will notice that in each test item one of the five is underlined. You are to look at the one combination that is underlined, find the same one after that item number on the Arswer Sheet, and fill in the circle under it.

The following examples have been marked correctly on your Answer Sheet. Note that the combination marked on the Answer Sheet must be exactly the same as the one that is underlined in the test item.

\section{Examples}
V. $A B$ AC $A D$ AE $A F$
W. $a A$ aB BA Ba Bb
X. AT 7 A 87 7B AB
Y. Aa Ba bA BA $\mathrm{BB}$
Z. $\begin{array}{lllll}3 A & 38 & 33 & 83 & 88\end{array}$

If you finish the items in Part I before time is called, check your work. Do not turn to Part II until you are told to do so. Work as fast as you can.

You will have 3 minutes for each part of this test. Work as rapidly and as accurately as you can. If you are not sure of an answer, mark the choice that is your best guess.

DO NOT TURN THE PAE- UNTIL YOU ARE TOLD TO DO SO. 


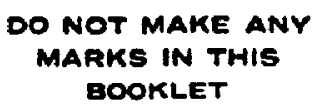

\section{MECHANICAL REASONING}

MARK YOUR ANSWER' ON THE EEPARATE ANSwER BHEET :

Find the space for Mechanical Reasoning on the Answer Sheet.

This test consists of a number of pictures and questions about those pictures. Look at the two examples below, to see just what to do.

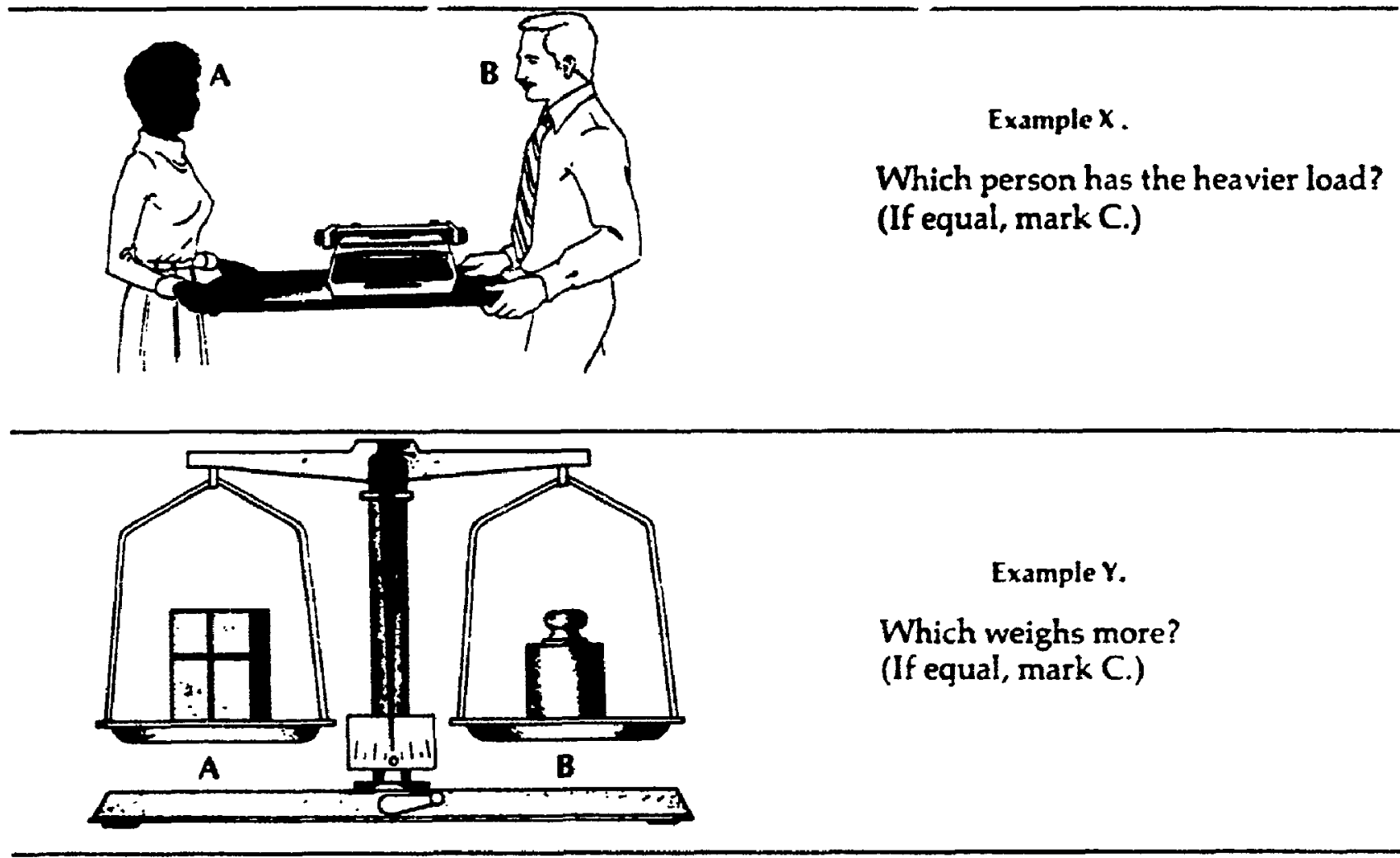

Example $\mathrm{X}$ shows a picture of two people carrying a typewriter on a board and asks, Which person has the heavier load? (If equal, mark C.) Person B has the heavier load because the weight is closer to him than to person $A$. Therefore, the circle for $B$ has been filled in on line $X$ of your Answer Sheet.

Now do the next one, Example $Y$, yourself. Mark the correct space on line $Y$ of your Answer Sheet. Example $Y$ asks, Which weighs more? (If equal, mark C.). As the scale is perfectly balanced, $A$ and $B$ must weigh the same, so you should have filled in the circle for $C$ on line $Y$ of your Answer Sheet.

On the following pages there are more pictures and questions. Read each question carefully, look at the picture, and mark your answer on the Answer Sheet. Do not forget that there is a third choice for every question.

You will have 30 minutes for this test. Work as rapidly and as accurately as you can. If you are not sure of an answer, mark the choice that is your best guess. 


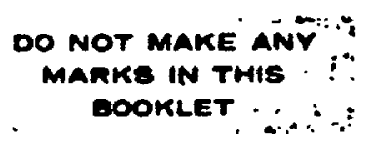

\section{SPACE RELATIONS}

\author{
DIRECTIONS
}

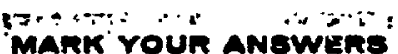

ON THE eEPARATE ZMANOWEN SHEET

Find the place for Space Relations on the Answer Sheet.

This test consists of 60 patterns which can be folded into figures. To the right of each pattern there are four figures. You are to decide which one of these figures can be made from the pattern shown. The pattern always shows the outside of the figure. Here is an example:

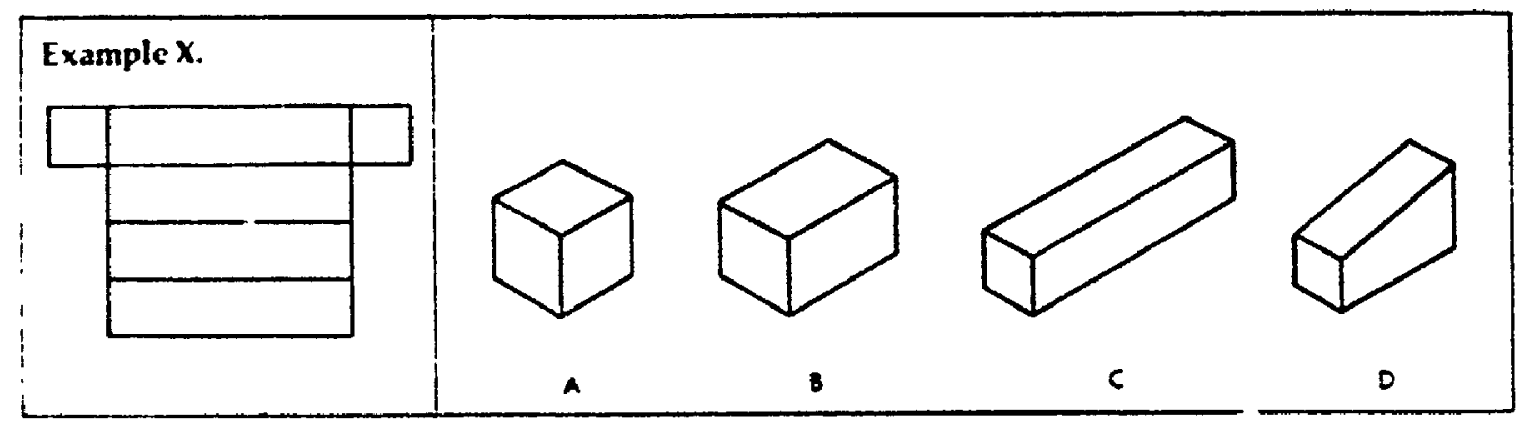

In Example $X$, which one of the four figures-A, B, C, D-can be made from the pattern at the left? $A$ and $B$ certainly cannot be made; they are not the right shape. $C$ is correct both in shape and size. You cannot mi xe D from this pattern. Therefore, the circle for $C$ has been filled in on line $X$ of your Answer $S$. set.

Remember: In this test there will always be a row of four figures following each pattern.

In every row there is only one correct figure.

Now look at Example $Y$ on the next page. 
Appendix B-3.2

LAMB Subtests 


\section{LIST LEARNING .. LEARNING TRIALS}

Eict Trial. "Tbis is a lest 10 see bow well you can remember several words. I am going 10 read you a list of 15 words. After I am linisbed I want you to tell me all of the words you can remember. The order you remember them does not matter.

"Each word represents a different type of word grouping or category. For example, it I said "waltz" and "trout". "waltz" would represent a type of dance, and "trout" comes from the fish category. Each of the 15 words I will read comes from one of the categories on this list. Present the category list. Read each category. then iemeve lis:.

You will be given 5 times to learn the 15 words. The first time 1 will read all 25 words. This is the andy time 1 will read the complete list. The rest of the time $t$ uill say only the words you omitted or said wrong. Then I'll ask you to tell me the complete list just as you did the first time." Read list of words .2 sec. interval between words.

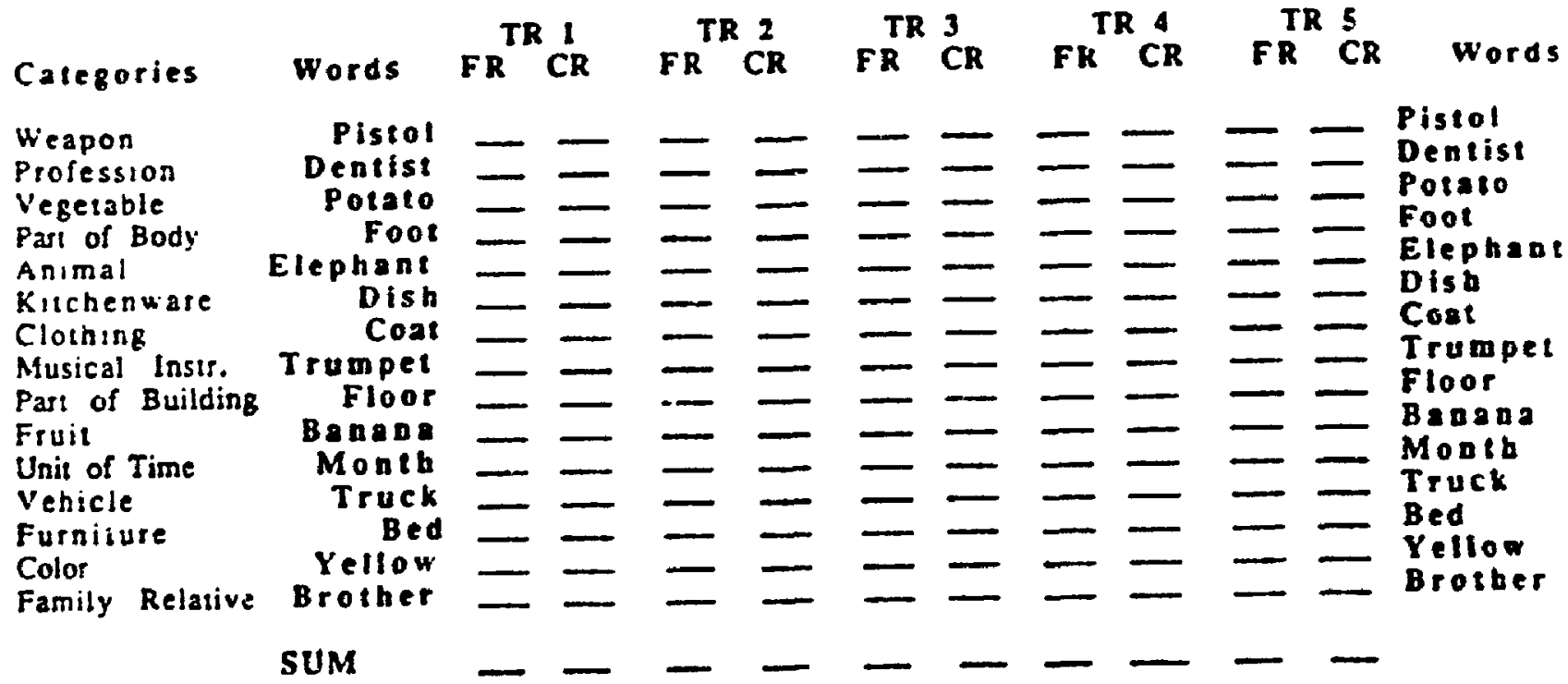

Exer-Reaall. "Tell me all of the words you can remember".

Cued-Recall. I $\mathrm{am}$ going to give you a litsle belp with the words you missed. Tell me the word that goes with the category which word was a type of w etc." For each answer respond with correct or incorrect. but do net provide the correct answer biemory Feedback. Tell person how many correct answers were achieved without help (freerecall), and with help (cued-recall), then go on to next trial.

Remaining Trials 2.5 or 2 consecuture crrorless ER irials).

If a person's combined FR and CR recall was less than 15 (less than 100\%) give the following instructions: "I am going to tell you the words you missed on the preceding trial. When I am through I want you to tell me ALL of the words you can remember from the entire list. including those you previously recalled correctly." Read only words that were incorrect on both FR or CR portion of previous trial (i.e. do not any read word that was correct on either FR or CR). Give feedback before reading words for next trial.

If a person's combined FR and $C R$ recall was is (100\%). give the following directions: "Since you remembered all of the words, I will not read you any words this time. Please tell me again all of the words you can remember from the list". 


\section{COMPLEX FIGURE .. LEARNING TRIALS}

Trial 1. "This is a test of how accurately you can remember a complex design. I am going 10 show y:u a design which bas lots of parts. After 1 bave given you 30 sec. to look at it. I'll remove it and ask you to draw it from memory. You will be given up to 2 minutes to $d r a w$ it as accurately as possible, but do not use a ruler. To help you keep track of time, every 30 sec. I will tell you bow much time is left.

I'll be showing the drawing to you times. This is the first time. Remember, don't start drawing until I take it away." The figure must be presented for 30 sec. even if person washes to look at it for less time. If this occurs say "Please continue looking at it ered if you feel you know it" Every $30 \mathrm{sec}$. inform person of amount of time left to complete the drawing. Record amount of time required to complete drawing.

Iriais 23 and 4. "Look at this ligure for 30 sec. and when I take it away I will give you up to 2 min. to draw it trom menuory." Every 30 sec. inform person of amount of time left to complete the drawing. Recotd amount of time required to complete the diawing.

Trial \#1: Score

Trial \#2: Score

Time

Time

(sec.)

Trial \#3: Score

Time

(sec.)

Trial \#4: Score

Time

(sec.)

Trial Ha: Score

(sec.) 


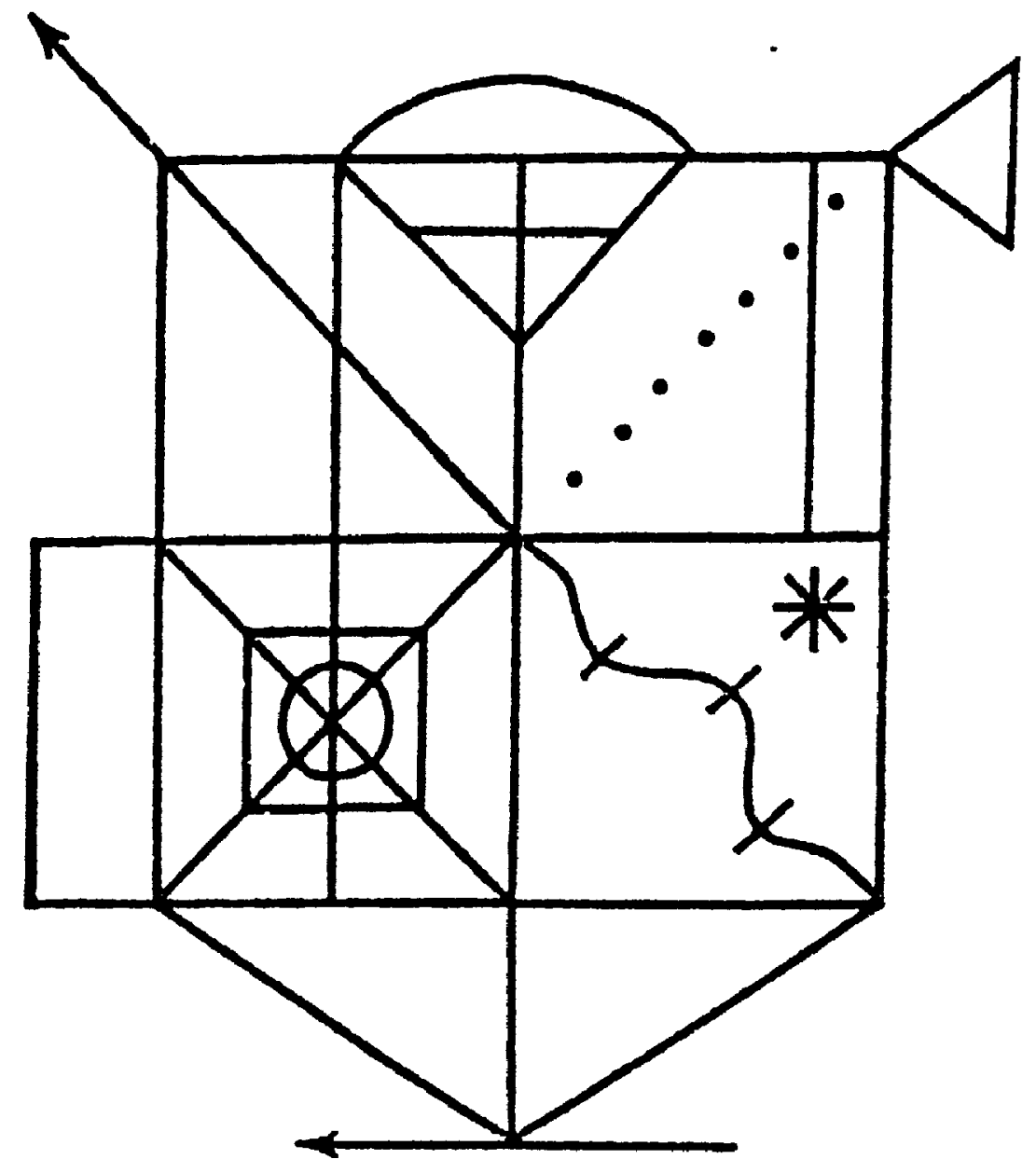




\section{LIST LEARNING .. RETENTION TRIAL}

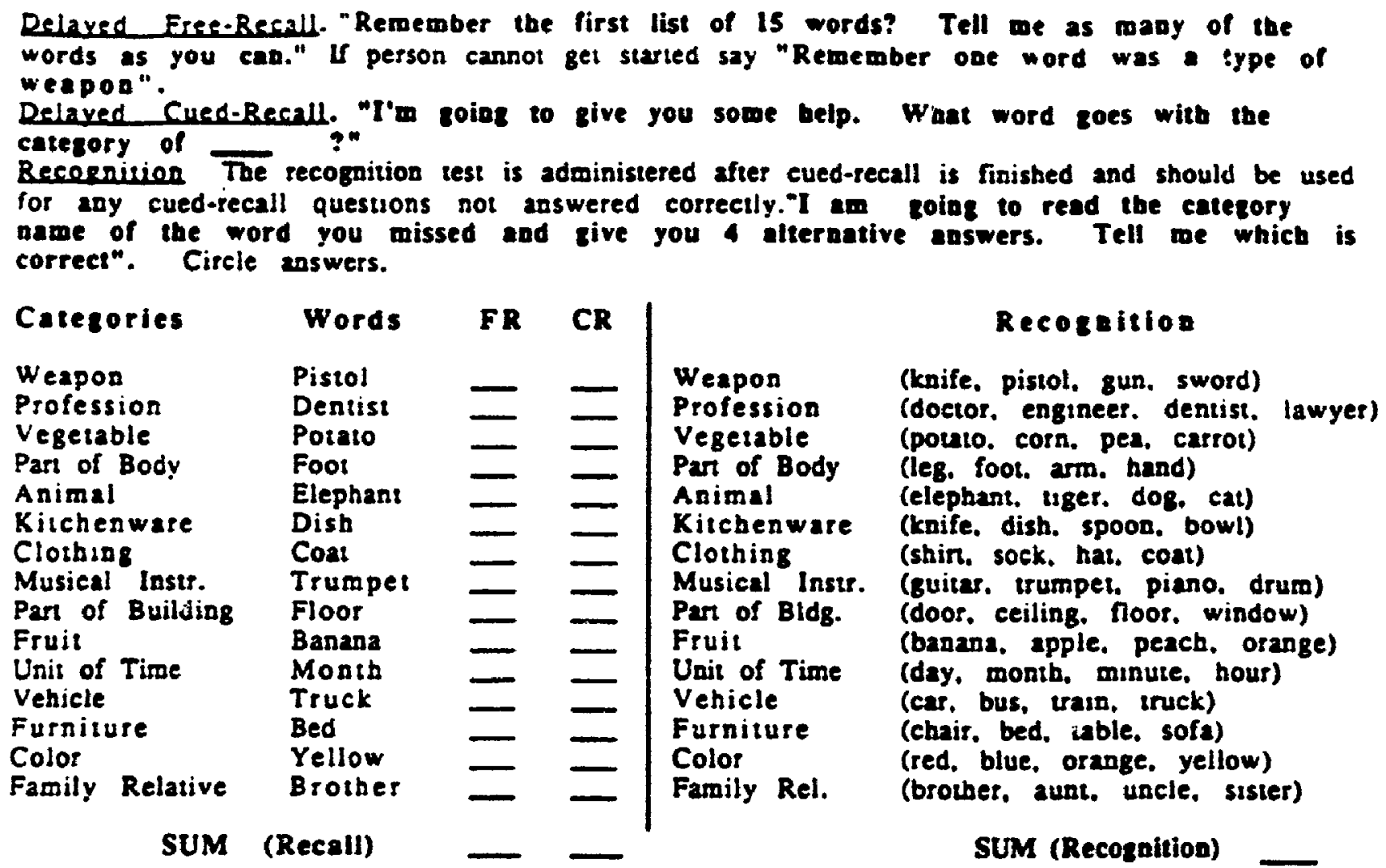

COMPLEX FIGURE - RETENTION TRIALS

Delaved-Recall. "Remember the complex figure that you saw earlier in the test. I would like you to draw as much of it as you can remember. You will have up to 2 miautes to do this." Every 30 sec. inform person how much time is left to complete the drav ing. Record the amount of time required to finish drawing.

Delayed Recall Trial: Score (sec.) 
WORKING MEMORY

. 2 sec or 3 sec / first or second presentation (Circle one from each set)

\section{Q-back condition}

In a moment I will be playing a tape with some recorded numbers on it. I would like you to repeat the numbers out loud as soon as you hear them. So if you hear 9 , you'll say 9 before I say the next number. If you hear 2, respond with a 2 right away. Even if you miss a number or fall behind, don't quit -- try to get back into the sequence. Okay? We'll begin.

\section{PRACTICE TRIAL (0-BACK)}

(On practice trial you may indicate when the number should be said by a nod of the head or some other gesture. However, do NOT do this beyond the third digit). Turn off tape after the practice trial. If person generally knows what to do , go to Trial 1 (person does not have to get all the answers correct -- merely has to demonstrate urderstanding of the general concept). If the person does not generally know, go over the card again. Then read the numbers outloud for a second practice trial. After the second practice trial go to Trial 1)

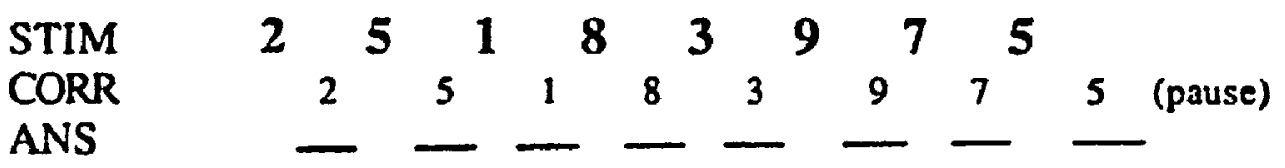

TRIAL 1 (0-BACK) I will turn on the tape again. As soon as you hear the number, say it out loud. (Write down every. mber. If person does not respond, place a dash (-). Do not leave any spaces blank. Do NOT give any help)

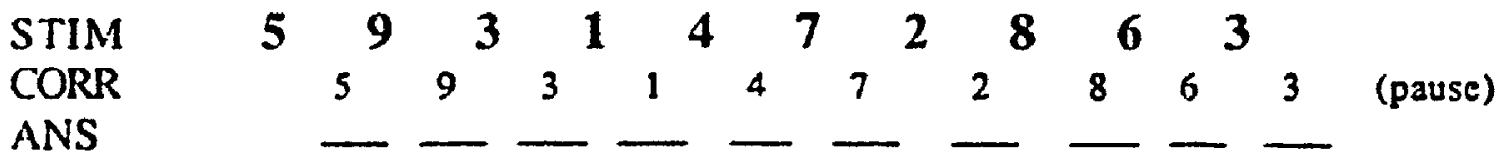

TRIAL 2 (0-BACK). Let's try it again. Repeat the number as soon as you hear it.

\begin{tabular}{|c|c|c|c|c|c|c|c|c|c|c|c|c|c|c|c|c|}
\hline STIM & 9 & 5 & & 1 & 7 & & 3 & & 3 & 2 & & 6 & & 4 & & \\
\hline $\begin{array}{l}\text { CORR } \\
\text { ANS }\end{array}$ & & 9 & 5 & & 1 & 7 & & 3 & 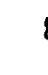 & & & 2 & 6 & & 4 & 9 \\
\hline
\end{tabular}

1-Back condition

Shortly you will hear another list of numbers. This time 1 want you to give me the number 1-back from the one you just heard. When you hear the first number. don't say anything. When you hear the second number, say the first number. Then after you hear the third number. say the second number and so on. I always want you to tell me the number that came one before the number just presented.

Here is an example. Let's say the tape says 85716 (Show card). When you hear 8, say nothing. When you hear 5 you say 8 . When you hear 7 , you say 5 . What do you say when you hear 2 ? (Ans 7). 
I will now turn on the tape for tise practice trial. Remember, do not say any number until you have heard the second number. If you fall behind or miss a number, do not quit -- try to get back inis the correct sequence.

\section{PRACTICE TRIAL (1 BACK)}

(On practice trial you may indicate when the number should be said by a nod of the head or some other gesture. Howevi:r, do NOT do this beyond the fourth digit). Turn off tape after the practice trial. If pe:son knows what to $\mathcal{L}:$ go on . If not. go over the practice card. Then read the numbers outloud for a second practice trial. and then go to Trial 1)

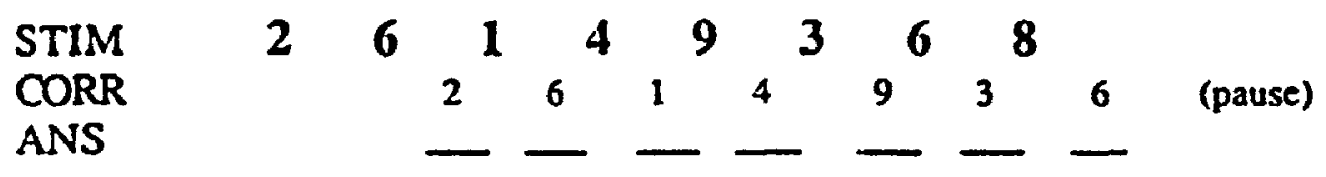

\section{TRIAL 1 (1-BACK)}

I will turn the tape on again -- remember do not give any responses until you have heard the second number. Then tell me the number that was 1 before it. If you fall behind, don't give up -- try to get back into the sequence again. (Do NOT give any gestures to help.)

STIM

CORR

ANS $\begin{array}{llllllllllllllllll}9 & 2 & { }^{6} & 8 & 5 & 1 & 3 & 7 & 4 & 2 & 9\end{array}$ - -2

TRIAL 2 (1-BACK) Let's try it again. Remember do not say any number until you have hearad the second number. Then tell me the number that was 1 back.

S IIM

.CORR

ANS

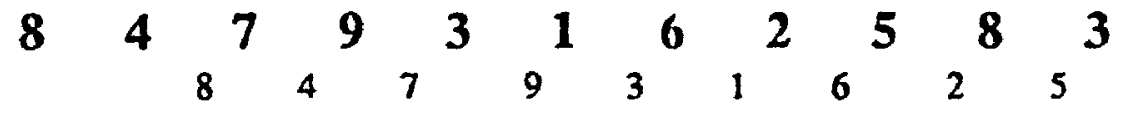

8 (pause)

\section{2-Back condition.}

This time I want you to give me the number 2 back from the one you just heard. Thus you are to say nothing when you hear the first two numbers. Then when you hear the third number. say the first number. When you hear the fourth number, say the second number and so on. I always want you to tell me the number that came two before the number being presented

Here is an example. Let's say the tape says 85716 (show card). Do not say anything antil you hear the third number which is 7 . When you hear 7 , then you say 8. When you hear 1 , you say 5 . What do you say when you hear 6 ? (Ans $=7$ ).

I will now turn on the tape for the practice trial. Remember, do not say any number until you have heard the third number. 
PRACTICE TRIAL (2-BACK)

(On practice trial you may indicate when the number should be said by a nod of the head or some other gesture. However, do NOT do this more than once). Turn off tape after the practice trial. If person knows what to do, continue to Trial 1 . If the person does not know, go over the card again. Then go directly to Trial 1 .

STIM

CORR

$$
26
$$

ANS

\section{TRIAL 1 (2-BACK)}

I will turn the tape on again -- remember do not give any responses until you have heard the third number -. then tell me the number that was 2 before it. (Do NOT give any gestures to help.)

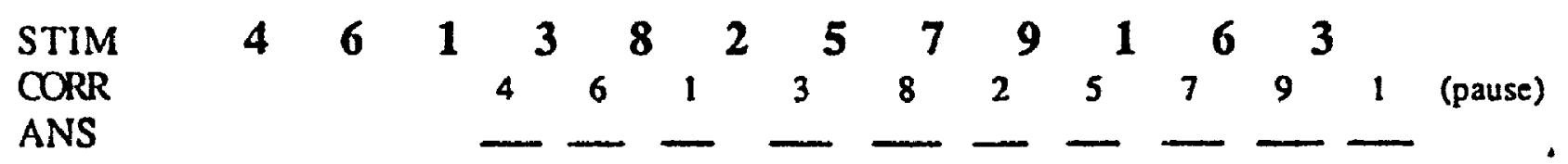

TRIAL 2 (2-BACK) Here is a another trial. Do not give any responses until you have heard the third number. Then tell me the number that was 2 before it.

STIM

$$
24
$$

CORR

ANS

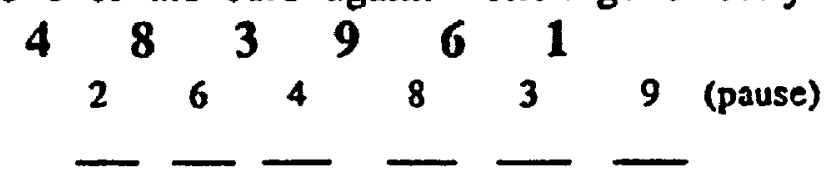

3-Back condition (Administer ONLY if they got at least the first 3 correct on either trial above)

This time I want you to give me the number which is 3 back from the one you just heard. Thus, you will not say anything before the fourth number. Then you will say. the number that occurred 3 before it.

Look at the card again. Let's say the tape says 85716 . Do not say anything until you hear the fourth number which is 1 . When you hear 1 , then you say 8 . Then when you hear 6 , you say 5 .

I will now turn on the tape for the practice trial. Remember, do not say any number until you have heard the fourth number. 
PRACTICE TRIAL (3-BACK)

(On practice trial you may indicate when the number should be said by a nod of the head or some other gesture. However, do NOT do this more than once.). Turn off tape after the practice trial and go to trial 1)

$\begin{array}{llllllllllllllll}\text { STIM } & 2 & 6 & 4 & 8 & 3 & 9 & 6 & 1 & \\ \text { CORR } & & & & & 2 & 6 & 4 & 8 & & 3 & \text { (pause) } \\ \text { ANS } & & & & & & & & & & & & & & & \end{array}$

\section{TRIAL 1 (3-BACK)}

I will turn the tape on again -- remember do not give any responses until you have heard the fourth number -- then tell me the number that was 3 before it. (Do NOT give any gestures to help.)

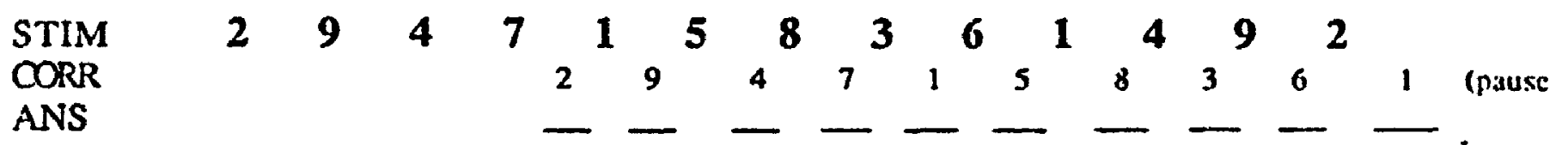

TRIAL 2 (3-BACK). Here is anouther trial. Do not give any responses until you have heard the fourth number. Then tell me the number that was 3 before it.

$\begin{array}{lllllllllllllllllllll}\operatorname{STIM} & 3 & 1 & 5 & 8 & 6 & 9 & 4 & 7 & 2 & 8 & 5 & 1 & 3 & & \\ \operatorname{CORR} & & & & & 3 & 1 & 5 & 8 & 6 & 9 & 4 & 7 & 2 & 8 & \text { (pause) }\end{array}$

ANS 


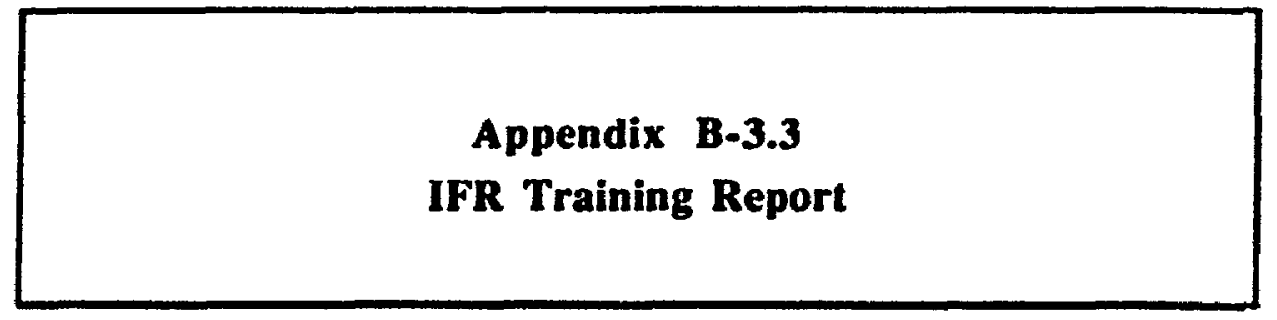




\section{IFR TRAINING REPORT}

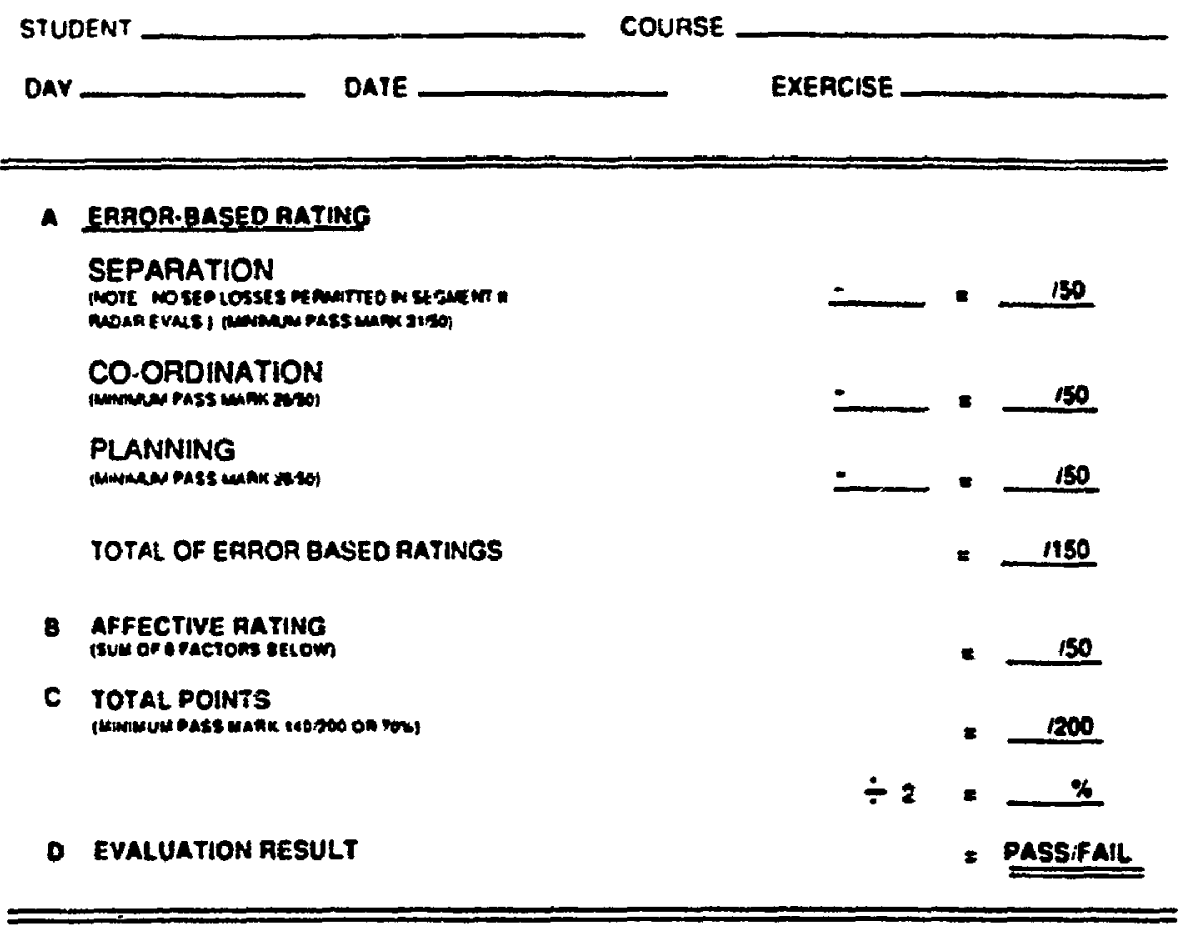

NOTE: SCORES ASSIGNED IN THIS AREA ARE FOR THIS EXERCISE ONLY

\begin{tabular}{|c|c|c|c|c|c|}
\hline $\begin{array}{c}\text { AFFECTIVE } \\
\text { FACTORS }\end{array}$ & \multicolumn{4}{|c|}{ RATINGS (TICK ONE ONLY FOR EACH FACTOR) } \\
\cline { 2 - 6 } & 4 & 3 & 2 & 1 & COMMENTS \\
\hline
\end{tabular}

\begin{tabular}{|c|c|c|c|c|c|}
\hline $\begin{array}{l}\text { Separation } \\
\text { visualization }\end{array}$ & 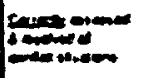 & 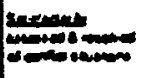 & م= & (5) & \\
\hline $\begin{array}{l}\text { Planning } \\
\text { Pnortives }\end{array}$ & $\mid$ & $\sin _{x \rightarrow \infty}$ & : & $\lim _{\infty}$ & \\
\hline $\begin{array}{c}\text { Bosid } \\
\text { Mznagement } \\
\text { Strupwntung }\end{array}$ & (1) & 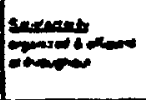 & 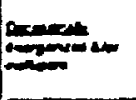 & Lentim & \\
\hline $\begin{array}{c}\text { Phraseolooy } \\
\mathbf{1} \\
\text { Communications }\end{array}$ & toms & manters & $\lim _{-\infty}$ & Emaren & \\
\hline $\begin{array}{c}\text { Co-ordination } \\
\text { Teamurotk }\end{array}$ & t & 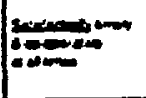 & 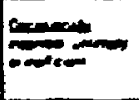 & $L_{\min }$ & \\
\hline $\begin{array}{l}\text { Confidence } \\
\text { Comportment }\end{array}$ & - & 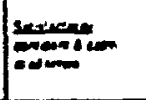 & $\lim _{x \rightarrow \infty}^{0+\infty}$ & $\lim _{x \rightarrow \infty}$ & \\
\hline
\end{tabular}

TO (B) ABOVE. AFFECTIVE RATING = $\times 2=\$ 50$

NARRATIVE

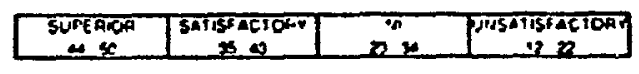

STUDENT SIGNATURE

INSTRUCTOR SIGNATURE 

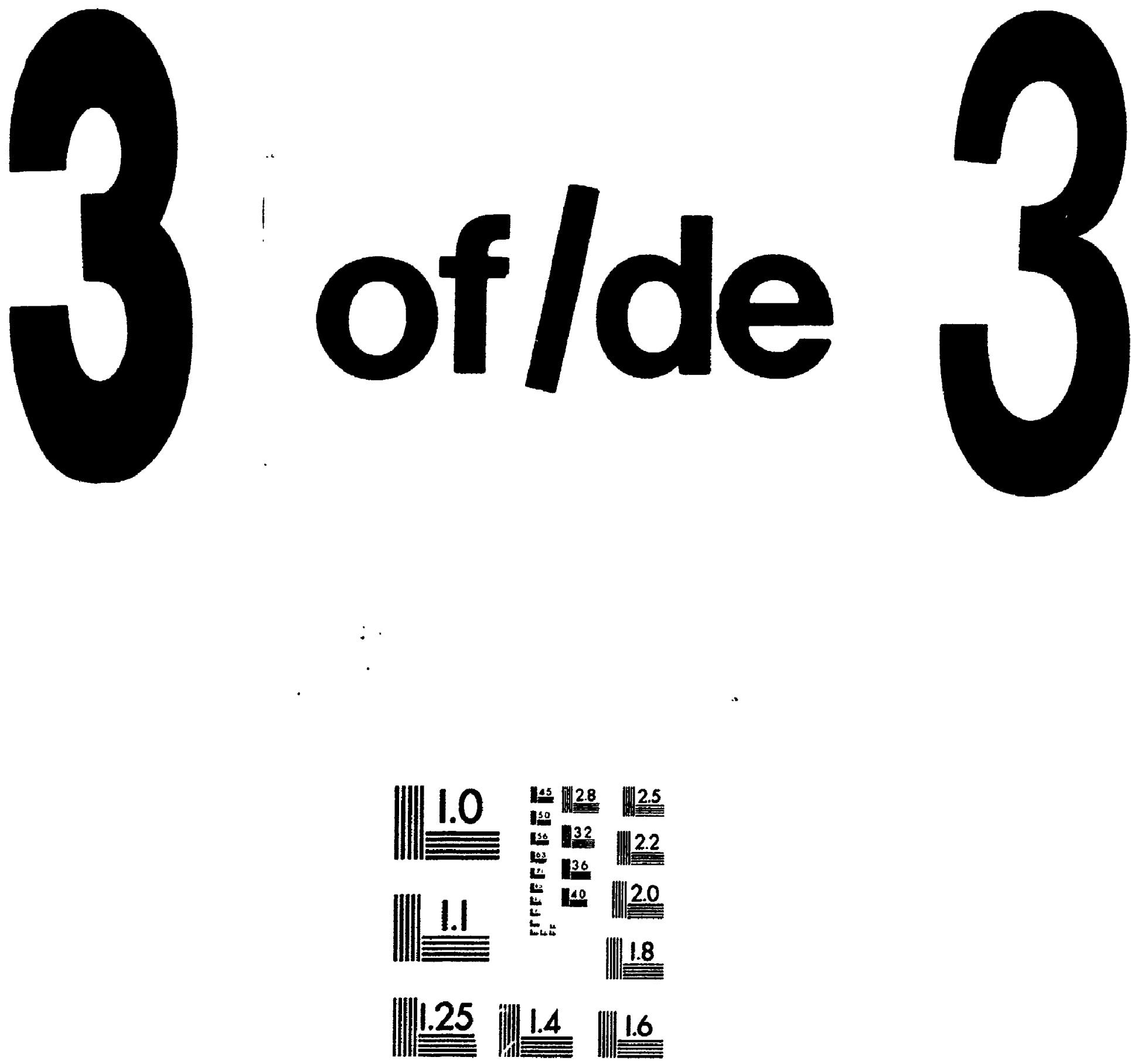

MICROCOPY RESOLUTION TEST CHART

NATIONAL BUREAU OF STANDARDS

STANDARD REFERENCE MATERIAI. 1010a

(ANSI and ISO TEST CHART No 2) 


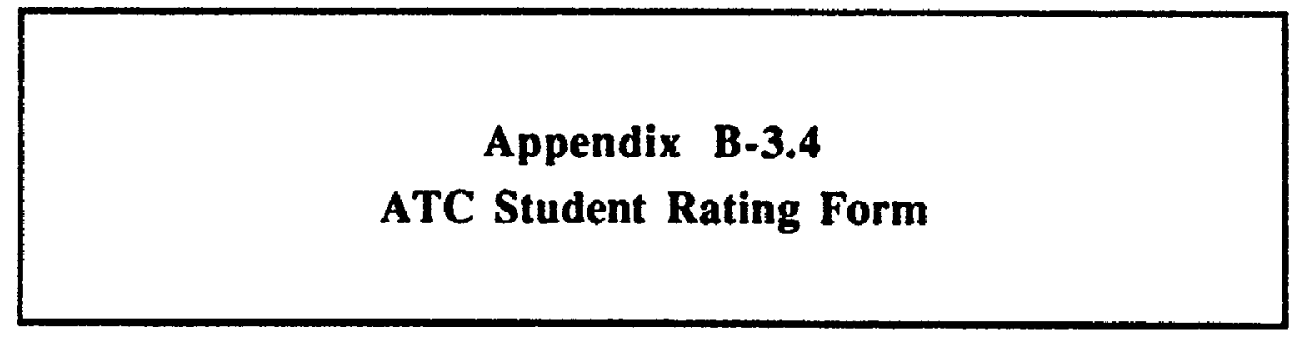




\section{ATC STUDENT RATING FORM}

NAME OF STUDENT

DATE

IN EVALUATING THE STUDENT, TRY TO CONSIDER HIS/HER PERFORMANCE DURING THE BUSIER PERIODS AS MUCH AS POSSIBLE. PLEASE TAY TO BE REALISTIC IN MAKING YOUR RATINGS. EVERYONE CANNOT BE AVERAGE OR EXCEUENT INEVERYTHING.

\section{Knowle \\ 2. Comprehention}

(a) Distinguishing between routine and potentially dangerous situations

(b) Getting and holding the picture.

\section{Judgement}

Making wise, appropriate decisions (vs. rash or nonappropriate)

\section{Communications}

(a) Use of standard phraseology

(b) Delivery technique

(c) Clarity and understandability

(d) Quantity (too much? too little?)

(a) Timing

\section{Iraffic Meneqement Techniques}

(b) Capacity to move traffic

(c) Command of situations

(d) Prevention of delay through good control techniques

(e) Planning provides sufficient safoty (versus last minute corrections)

$$
\text { 6. Performence Under Sirese }
$$

(a) Recognizing all aspects of a traftic situation

(b) Self-confident but aware of his/her limitations

(c) Ability to remain calm

(d) Ability to remain tactul

(e) Consistency in safe separation

(a) Accomplishes at proper time

(b) Overall elfectiveness (understandable, complete)

8. Generd Releted Elements of ATC Performance

(a) Ability to work effectively with others

(b) Willingness to assume his/her share in teamwork

(c) Acceptance of suggestions and criticism

(d) Tactulness in making appropriate suggestions to others

(e) Interest and pride in work

(t) Efforts toward self-improvement

(g) Steady attention to work and conduct

(h) Adaptability to changes in policies, procedures, eic.

(i) During period of peak traftic or adverse weather, to what extent is

the controller capable of meeting the demands of the "hottest" positions?

\section{Ovarnll Relative Bating}

Use the scale below to rate the overall proficiency it this ATC student relative to all the ATC students you have known. (Indicate your rating by placing a check mark in the appropriate box.)

\begin{tabular}{|c|c|c|c|c|c|c|}
\hline $\begin{array}{c}\text { Top } \\
10 \%\end{array}$ & $\begin{array}{c}\text { Upper } \\
\text { Intermediate } \\
15 \%\end{array}$ & $\begin{array}{c}\text { Upper } \\
\text { Middle } \\
15 \%\end{array}$ & $\begin{array}{c}\text { Middle } \\
20 \%\end{array}$ & $\begin{array}{c}\text { Lower } \\
\text { Middle } \\
15 \%\end{array}$ & $\begin{array}{c}\text { Lower } \\
\text { Intermediate } \\
15 \%\end{array}$ & $\begin{array}{c}\text { Bottom } \\
10 \%\end{array}$ \\
\hline & & & & & & \\
\hline $100 \% \quad 90 \%$ & $75 \%$ & $60 \%$ & $40 \%$ & $25 \%$ & $10 \%$ & $0 \%$
\end{tabular}


Appendix B-4.

Preliminary Study

B-4.1: Experimental Protocol 


\section{INTRODUCTION}

At the outset of the experiment, the $S$ is seated before the screen and mouse. The simulation has been loaded and the TRACON radar screen with impending flight strips is visible. The second screen and keyboard are used by the experimenter. Another table is located at 90 degrees to the $S$ so that the $S$ must turn away from the screen to perform tasks on it. A plotting board with a map on it is placed on the table. The plotting markers are not visible to the $\mathrm{S}$.

The $\mathrm{E}$ says:

"This experiment is designed to find out people use information when performing the task of air traffic control. In this experiment, we will use the air traffic control simulation program you see before you. The screen is organized into a radar section, a flight data strip section and a communications section. The first line of the flight data strips shows aircraft identifier, type, planned (not actual) airspeed and altitude. The second line shows the flight plan of the aircraft. It is an overflight if the flight plan ends in "ctr." and an arrival if it includes "YYZ24L or R".

You will issue all commands to the aircrafi by voice and I will enter the commands using the keyboard. The only control you will need will be the mouse; this controls the placement of aircrafi data tags to avoid overlap and screen clutter. To use the mouse, point the mouse cursor to the aircraft symbol on the radar screen and then click and hold the left mouse button. Drag in the direction you want the data tag to go and release the mouse button.

The table to the right will be used when you are requested to make responses. On it you can see a map which is laid out the same as the sector you will be working with in the simulation. You will be using this map later. To your right on the same table as the monitor is real sector map you may use for reference.

The radar background represents the Toronto sector. You will be controlling arrivals to two runways, 24 left and 24 right. Arrivals will always use approaches through SUTAR, GOVIX, DAVNI or AVTON (use diagram). Arrivals from the north will use only the right runway and arrivals from the south will use only the lefi runway. You may issue commands one at a time to vector aircraft or issue a series of commands to be automatically execused in sequence.

Flights entering through GOVIX must be maintained above 4000 feet until past the Buttonville airport. Flights entering through AVTON must maintain at least 4000 feet until inside the inner arrival box. Arriving aircraft must be vectored so that they are within 30 degrees of the runway heading (236 magnetic) and at the proper approach altitude (2000 feet). When handed off, the aircraft will correct its heading to the runway but should not be handed off until it has reached 12 miles DME. You may instruct an aircraft to turn on to the localizer (the solid line) but it must be at the correct runway heading and altitude by the time it crosses over the gap (outer marker) onto the dashed line.

Overflights will also be entering and passing through the airspace. In most cases of overflights, you will not have to do anything with them. However, the experiment requires that pilots of overflights may sometimes request altitude changes. You may approve or modify these requests. 
You may ask for a map of fixes, information on aircraft characteristics or a report of an aircraft's speed and heading at any time (demonstrate). The data tags on this simulation show only altitude and do not include ground speed.

At certain times during any one of the simulations in this experiment, pilots may make errors of the following kinds:

1 pilots may repeat directions back correctly but immediately do the wrong thing.

2. pilots may make an unrequested change in altitude

3. pilots may make an unrequested change in heading.

Your task is to watch for such problems and, immediately upon detecting one, contact the pilot to correct the problem. However, these problems will not occur frequently.

The first phase of the experiment will be practice with the system. For about 20 minutes, you will control a series of arrivals and overflights. You may ask to pause the simulation at any time to ask questions."

\section{RECALL AND CATEGORIZATION CONDITIONS}

After the subject has completed the practice session satisfactorily (it may be repeated if necessary) the E starts the "CATEG.SIM" module, pauses the simulation and says:

"Now another series of aircraft will be coming into your sector. At a certain point I will pause the simulation and ask you to recall what was on the screen. Then you will be able to look at the aircraft again and will be asked to make some judgements about them.

The simulation will begin in a minute. After about 15 minutes, I will pause the simulation and turn off your screen. As soon as I do this, immediately turn to the table at your right and draw as many aircraft as you can rememiber on the sector map. Show each aircrafi's position by $a$ dot and its heading by an arrow. Write beside it the identifier, altitude and speed. Here is an example of how it should look (show example). Do this as quickly and as accurately as possible.

The simulation is run ahead until aircraft TGO:42 has just become active.

At this point the E pauses the simulation and says:

"As soon as I turn off your screen draw all that you can remember from it as quickly and accurately as possible on the sector map on your right. Include aircraft location (shown by a dot), heading (shown by an arrow), ACID, altitude and speed."

The $\mathrm{E}$ turns off the $\mathrm{S}$ 's display and checks that the $\mathrm{S}$ has started. The $\mathrm{S}$ should not be allowed any time to study the display. The $E$ then records the actual altitudes, speed and heading for each aircraft from the display while the $S$ works. Order and timing information are taken from the video tape.

As soon as the $S$ is finished, the $E$ turns the $S$ 's monitor back on and says:

"Now I want you to make comparisons between the aircraft which are presently on the screen. I will give you some pages on which all the possible pairs of aircraft are listed. 
For each pair I would like you to compare the two aircraft in terms of their stoms in the air traffic control situation on the screen.

Across from each pair of aircraft is a line with "same" and "different" at each end. If the two circraft are exactly the same in terms of their status, draw a vertical bar as close as possible to the word "same". If they are completely different, draw a vertical bar as close as possible to the word "different". Look at the first two lines on the page for examples.

If the aircraft are somewhat similar or somewhat different, draw a vertical bar crossing the line somewhere in between the extremes of "same" or "different" according to how similar or dissimilar the aircraft are with regard to their status. See the third example on the page.

There is no time limit for this task, so you may take your time to make each evaluation. You may look at the screen while doing this task."

After the $\mathrm{S}$ has finished making the comparisons, the $\mathrm{E}$ says:

"Now that you have had some time to work with the simulation and think about the traffic now on the screen, I would like you to describe the situation you see before you."

The S's response is recorded on video tape for later review. The $E$ then says:

"On the sheet of paper I am now going to give you is another list of pairs of aircraft. This time, instead of rating their similarity, I would like you to draw a line showing how far you judge they are apart from the display in front of you. I will give you the pair that are the farthest apart first so that you may scale your line appropriately to the space provided. Then go ahead an draw lines showing how far each pair of aircrafi are apart. Use a consistent scale during this exercise. You may go back and make corrections, but there is no need to spend too much time on each judgement."

\section{SPATIAL CONDITIONS}

\section{NEW MAP}

The E the loads the "SPATPRAC.SIM" module and puts the simulation on pause.

\section{A) SUBJECTIVE ALTITUDE JUDGEMENTS}

The $S$ is asked to turn to face the plotting board and the $E$ says the following:

"This is a map representing the control sector on the simulation screen. I would now like you to take a minute to think of the mental image or picture you now have of the airspace."

The E pauses to let the $S$ think about this...

"When your are ready, point to a level in the space above the map where you think the altitude of 20,000 feet would be. When you do this, show me the height you picture in your mental image of the airspace rather than your scale calculation of what it should be (unless these are the same, of course). When you have pointed to the correct height for you, hold your hand still for a few moments while I take a measurement." 
When the $S$ points to the level above the board representing 20,000 feet, the $E$ measures the height of the S's finger above the plotting board. This is repeated two more times.

Then, one position marker is then placed in the centre of the plotting board with the slider at the bottom. The E says:

"I will now ask you to position the slider on this plotting marker to a series of different altitudes. Although you may have previously shown me a larger or smaller height for the altitude of 20,000 feet, I would now like you to rescale your judgements so that 20,000 feet is contained below the slider. I would now like you to take a minute to imagine the airspace again with this scale adjustment. Also think abous where different altitudes in the airspace are in your mental image of the sector."

When the $S$ indicates readiness, the $E$ says:

"When I give you the following altitude quantities, move the slider to the height which would represent that height to you. To keep things consistent we will say that the distance betwees: the bottom of the slider and the plotting board is the altitude."

The $E$ then calls off the altitudes in the order found on the response form and measures the height of the bottom of the slider from the plotting board for each altitude. The slider is moved back to the bottom of the marker after each trial.

\section{B) PRACTICE IN PLOTTING SINGLE AND PAIRS OF AIRCRAFT}

\section{NEW MAP}

The E starts the "SPATPRAC.SIM" module and pauses the simulation.

The E now says:

"A practice run will now be started so that you may practice using the plotting board to show the present and future positions of single aircraft and pairs of aircraft. The simulation will be run until the aircraft are in a certain position. At some point I will ask you to show me the positions of a particular aircraft or two aircraft on the plotting board.

Sometimes two aircraft will be approaching each other on crossing tracks, overtaking or head-on and one of the aircraft will ask for an altitude change. If you are not sure that separation will be maintained, you may refuse the request or modify it. However, for the purposes of the experiment, the altitude change will occur anyway. This may result in a loss of separation, but the experiment is set up so that no collisions will occur as a result of such altinude requests. Nevertheless, I am interested in whether you see a possible problem or not, so deal with the requests as you normally would.

Euch time the simulation is paused, one or two aircraft will be specified and you should focus on it or them. Then you will then be asked to make a series of three plots of each aircraft's position. The first will be its present position, then its position 2 minutes in the future and then its position 5 minutes in the future. To plot an aircrafi's isisent and future positions, follow these steps: 
1. start with the present position and pick up a marker with a black slider and push it into the board. Push the marker well into the board to anchor it and use one hand to steady the marker while you move the slider.

2. position the sliaier to your estimate of the correct altitude.

3. if two aircraft are to be plotted, take the second marker with s. black slider and position it in the same way. If the two aircraft are in the same location, place the markers side-byside and draw a circle around both of them when you label them.

4. the next step is to place a marker where you think the aircraft will be in 2 minutes. Place a marker with red slider in the board and position the slider to estimate the altitude the aircraft will have in 2 minutes.

5. if there is a second aircraft to plot, use the second marker with a red slider for this purpose.

6. then plot the Gircraft's position in 5 minutes from its present position. Use a marker with a green slider and move the slider to indicate the altitude the aircraft will have.

7. if there are two aircrafi to plot, use a second marker with a green slider.

8. using the correct colour pen to match the colour of each slider, write the aircraft identifier and its altitude on the map beside each marker.

Please complete the plotting of aircraft position as quickly as possible, but keep in mind that accuracy is also important. Take as much time as you want with the labelling.

The simulation is run and paused when aircraft are in position to be plotted. The following list indicates where each aircraft should be when the simulation is paused:

1. ACA602 - after passes triangle.

2. UAL987 - just after enters inner arrival box and makes turn.

3. AAL416 and CDN230 - change altitude at triangle and pause at $5 \mathrm{~nm}$ apart.

The E says in the case of the single aircraft:

"Now I am going to give you one aircraft to plot. As soon as I say the name of the aircraft, I please focus on it on the screen and then turn and insert a marker with a black slider into the plotting board where you think the aircraft is presently located and move the slider to the altitude you think the aircraft is presently at. Then immediately take a marker with a red slider, position it and move the slider to where you think the aircraft will be in 2 minutes. Then take a marker with a green slider, position it and move the slider to where you think the aircraft will be in 5 minutes. After you have completed this plotting exercise, write the identifier and altitude of each aircraft on the map with the pen colour matching the slider colour.

1. The aircraft is ACA602

2.The airci aft is UAL987"

The $E$ times the period from giving the $S$ the target to when the second five minute marker and slider is placed. The identifiers and altitudes of the aircraft are noted on the map by the 
S. The number of times the $S$ turns to check the screen during the plotting phase is also recorded.

The $\mathrm{E}$ then removes the markers from the board, moves the sliders to the bottom of the marker and runs simulation ahead until the situation with two aircraft is ready. The E says:

"Now I am going to give you two aircraft to plot. As soon as I say the names of the aircraft, I want you to focus on them and then turn and insert markers with black stiders into the plotting board where you think the aircraft are presently located and move the sliders to the altitudes you think the aircrafi are presently at. Then immediately take markers with red sliders, position them and move the sliders to where you think the aircraft will be in 2 minutes. Then take markers with green sliders, position them and move the sliders to where you think the aircraft will be in 5 minutes. After you have completed this plotting exercise, write the identifier and altitude of each aircraft on the map with the pen colour matching the slider colour.

The aircraft are AALA16 and CDN230."

The $E$ times the period from giving the $S$ the targets to when the second five minute marker and slider is placed. The identifiers and altitudes of the aircraft are noted on the map by the $S$. The number of times the $S$ turns to check the screen during the plotting phase is also recorded.

After the last plot, the $\mathbf{E}$ runs the simulation ahead for 5 minutes of clock time to re:ord on tape the future positions of the last pair of aircraft (this may be done in fast time).

\section{C) EXPERIMENTAL TRIALS: SINGLE AIRCRAFT}

\section{NEW MAP}

The E starts the "SPATONE.SIM" module, pauses the simulation and says:

"Now a number of aircraft will cross the screen on overflights or on arrival At certain times the simulation will be paused and you will be asked to plot the present and future positions of each aircrafi as you just did. Please do not initiate changes in the altitudes or speeds of overflights although pilots may occasionally ask for such changes. As I mentioned earlier, please react to requests for altitude changes as you normally would. However the changes will be executed anyway so that the requirements of the experiment can be met. No collisions are programmed to occur, although loss of separation might happen occasionally."

The $E$ runs the simulation and pauses it when a target is in the desired position. The $E$ proceeds as in the practice sessions. The position information for these aircraft is:

1. SWR134 - change altitude at triangle and pause 2 miles later.

2. CDN221 - pause after it leaves the end of the airway.

3. KLM871 and ONT119- pause after first aircraft enters arrival box.

4. EAL410 and WWC423 - pause as first aircraft enters arrival box.

As the first aircraft comes into position, the simulation is paused and the $E$ says: 
"Now I am going to give you one aircraft to plot. As soon as I say the name of the aircraft, I want you to focus on it and then turn and insert a marker with a black slider into the plotting board where you think the aircraft is presently located and move the slider to the altitude you think the aircraft is presently at. Then immediately take a marker with a red slider, position it and move the slider to where you think the aircraft will be in 2 minutes. It will not be necessary to plot the five minute position. After you have completed this plotting exercise, write the identifier and altinude of each aircraft on the map with the pen colour matching the slider colour.

(After the first aircraft, the E may shorten the instructions and just give the aircraft name.)

1. The aircraft is SWR134.

2. The aircraft is $C D N 221$.

For the plots of the last two pairs of aircraft, the simulation is paused and the aircraft are both plotted as in the pairs of aircraft condition in the practice. The E says:

"For the next part of this exercise, I want you to plot a pair of aircraft. Please plot the present and future positions of two aircraft which are on arrival. Include both the two and five minute predictions. The simulation will remain on pause while you plot both of these aircraft.

3. The pair is KLM871 and ONT119.

4. The pair is EALA10 and WWC423."

The E times the period from giving the $S$ the target to when the second five minute marker and slider is placed. The identifiers and altitudes of the aircraft are noted on the map by the $S$. The number of times the $S$ turns to check the screen during the plotting phase is also recorded.

The $\mathbf{E}$ runs the simulation ahead for 2 minutes allowing the subject to control the last pair of aircraft in order to record their eventual positions.

\section{D) EXPERIMENTAL TRIALS: TWO AIRCRAFT}

The E starts the "SPATTWO.SIM" module, pauses the simulation and says:

"In this part of the simulation, as well as the usual flow of arrivals, pairs of aircraft will be approaching each other and I will ask you to plot their present and future positions. Some of the aircrafi on overflights will be changing altitude with respect to each other.

I will ask you not to initiate changes in the altitudes or speeds of overflights although the pilots may occasionally ask for such changes. You may refuse or modify such requests although for the requirements of the experiment, they will be initiated anyway. However, I am interested in your response to each situation, so handle it as you normally would. The simulation is set up so that no collisions will occur as a result of these altitude changes. However, loss of separation may happen."

The aircraft position information is:

1. WDA212 and TOR870 - change altitude at triangle and pause when $5 \mathrm{~nm}$ apart.

2. GPTL and USA340 - change altitude at triangle and pause at $5 \mathrm{~nm}$ apart. 
3. GDWS and AAL610 - change altitude at end of second airway and pause at $5 \mathrm{~nm}$ apart.

4. ACA908 and AAL860 - change altitude when AAL860 half way on airway and pause at $5 \mathrm{~nm}$ apart.

5. GNTZ and ACA474 - change altitude at triangle and pause at $5 \mathrm{~nm}$ apart.

When two aircraft are in position, the $E$ pauses the simulation and says:

"Now I am going to give you two aircraft to plot. As soon as I say the names of the aircrafi, I want you to focus on them and then turn and insert markers with black sliders into the plotting board where you think the aircraft are presently located and move the sliders to the altitudes you think the aircrafi are presently at. Then immediately tike markers with red sliders, position them and move the sliders to where you thisk site aircraft will be in two minutes. It will not be necessary to plot the five minute positions. After you have completed this plotting exercise, write the identifier and altitude of each aircraft on the map with the pen colour matching the slider colour.

1. The aircraft are WDA212 and TOR870.

2. The aircraft are GPTL and USA340.

3. The aircraft are GDWS and AAL610.

4.The aircraft are ACA908 and AAL860.

5.The aircrafi are GNTZ and ACA474."

The $E$ times the period from giving the $S$ the targets to when the second two minute marker and slider is placed. The identifiers and altitudes of the aircraft are noted on the map by the $S$. The number of times the $S$ turns to check the screen during the plotting phase is also recorded.

After the last plot, the $\mathbf{E}$ runs the simulation ahead for two minutes of clock time to record on tape the future positions of the last pair of aircraft (this may be done in fast time).

\section{FAULT DETECTION}

During the simulation conditions (all except SPATPRAC.SIM) the E will make the following pilot errors:

\section{PRACTICE.SIM}

1. make an unrequested change in heading to WWC650 (turn right 90 degrees).

2. immediately follow and altitude change command to AAL679 incorrectly (ascend to 15000).

\section{CATEG.SIM}

1. make an unrequested change in heading to CDN106 (tum left 90 degrees).

2. immediately follow an altitude change command to WWC714 incorrectly (ascend to 10000).

\section{SPATONE.SIM}

3. make an unrequested change in altitude to WDA816 (ascend to 15000).

4. make an unrequested change in heading to CDN221 (tum left 90 degrees). 


\section{SPATTWO.SIM}

5. immediately follow a heading change command to ACA709 incorrectly (turn loft to 270). 6. make an unrequested change in altitude to USA340 (ascend to 20000).

The time elapsed from the initiation of the problem to the S's initiation of a corrective measure is recordeci during the experiment or after from video tape. 


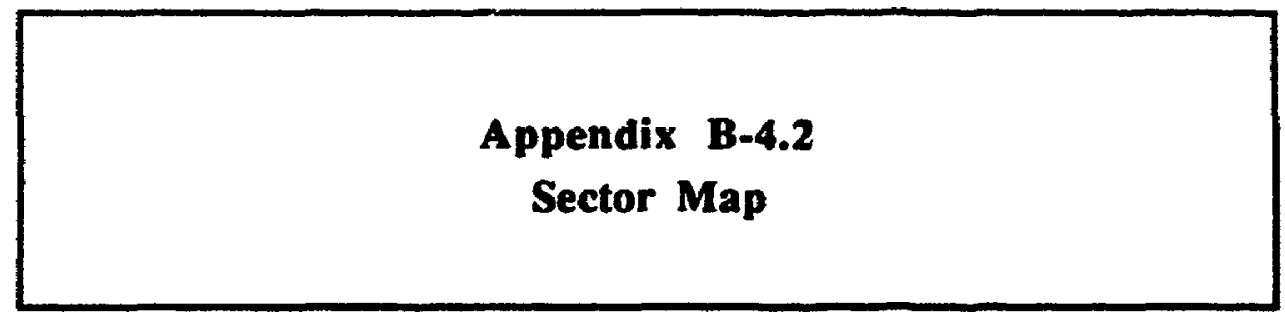




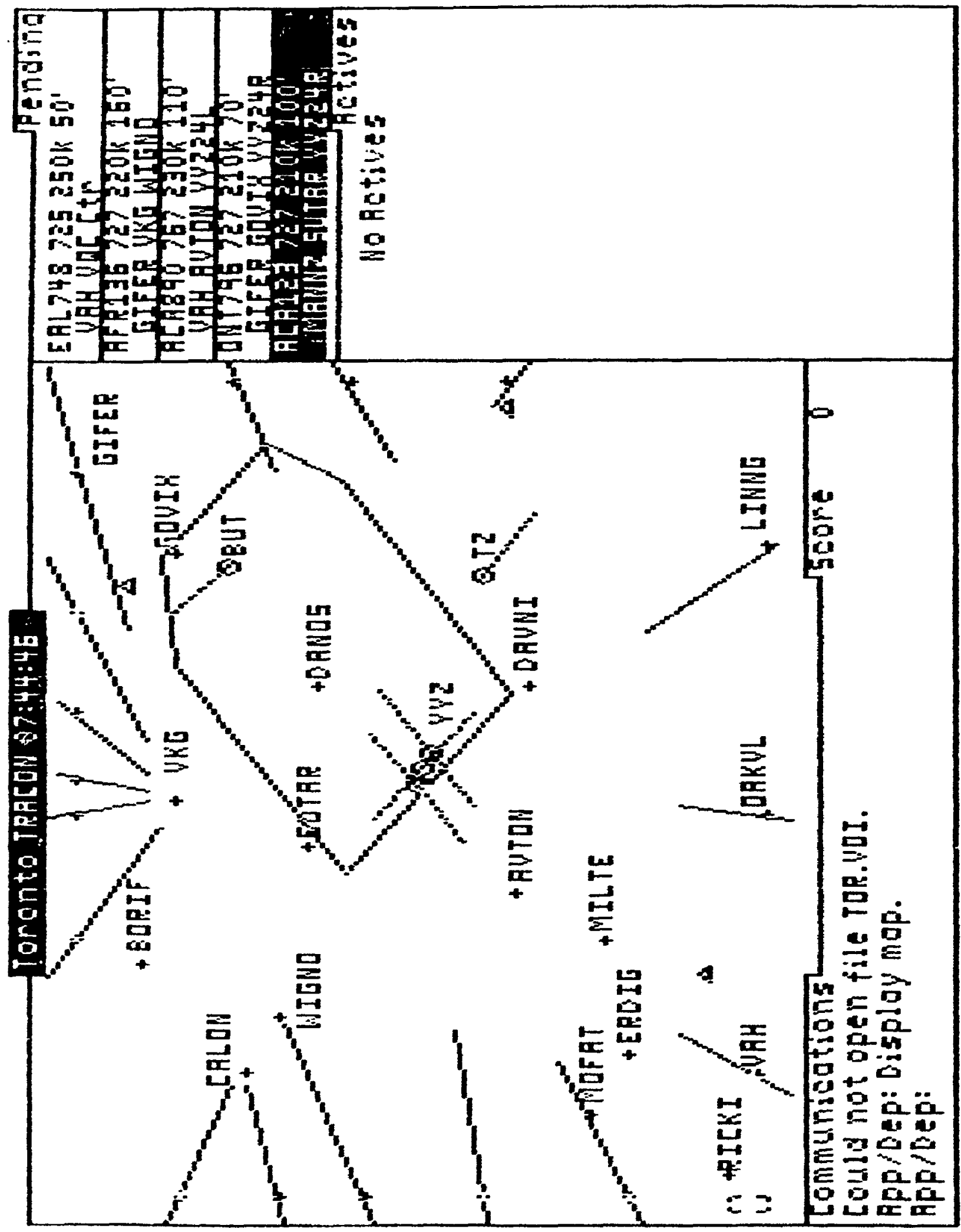




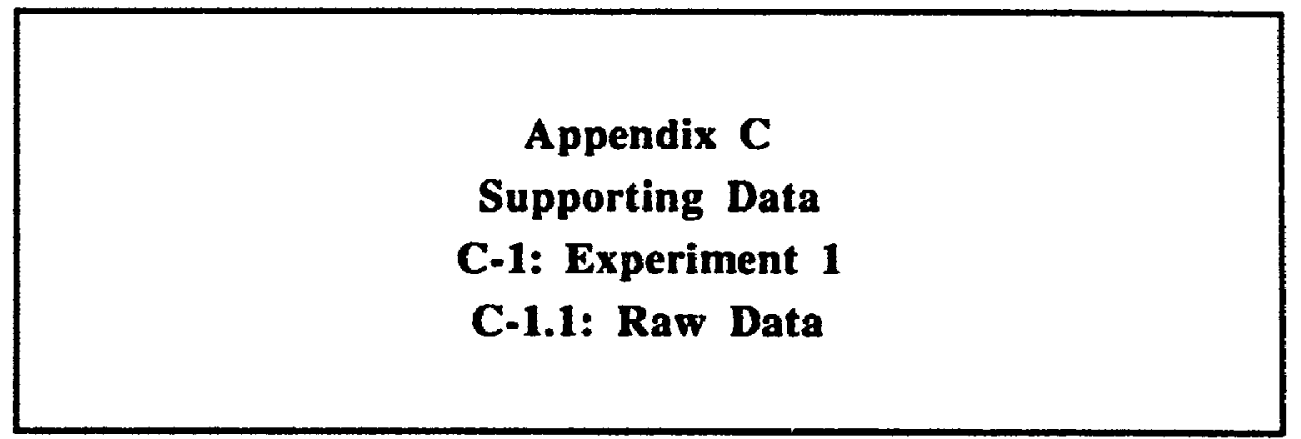




\begin{tabular}{|c|c|c|c|c|c|c|c|c|c|c|c|}
\hline$S$ & AGE & EDUC & MCAT & VR & NA & VR+NA & AR & CS\&A & MR & SR & FR1+FR2 \\
\hline 1 & 31 & 15 & 41 & 48 & 32 & 80 & 39 & 57 & 64 & 45 & 15 \\
\hline 2 & 33 & 15 & 43 & 48 & 39 & 87 & 44 & 58 & 65 & 49 & 19 \\
\hline 3 & 20 & 12 & 39 & 35 & 34 & 69 & 41 & 64 & 53 & 59 & 21 \\
\hline 4 & 30 & 18 & 45 & 46 & 38 & 84 & 42 & 51 & 68 & 57 & 15 \\
\hline 5 & 26 & 21 & 40 & 48 & 33 & 81 & 43 & 49 & 61 & 51 & 19 \\
\hline 6 & 28 & 16 & 38 & 38 & 39 & 77 & 39 & 70 & 76 & 57 & 26 \\
\hline 7 & 24 & 14 & 42 & 36 & 27 & 63 & 40 & 71 & 53 & 34 & 16 \\
\hline 8 & 26 & 17 & 46 & 48 & 39 & 87 & 44 & 51 & 62 & 58 & 11 \\
\hline 9 & 21 & 17 & 44 & 25 & 33 & 58 & 35 & 52 & 57 & 42 & 16 \\
\hline 11 & 27 & 17 & 42 & 49 & 38 & 87 & 45 & 61 & 62 & 55 & 16 \\
\hline 12 & 29 & 15 & 43 & 38 & 35 & 73 & 39 & 51 & 70 & 54 & 25 \\
\hline 13 & 30 & 16 & 43 & 44 & 35 & 79 & 40 & 51 & 65 & 39 & 21 \\
\hline 14 & 26 & 12 & 100 & 49 & 40 & 89 & 40 & 53 & 69 & 60 & 20 \\
\hline 15 & 35 & 12 & 44 & 48 & 38 & 86 & 45 & 58 & 63 & 59 & 21 \\
\hline 16 & 26 & 14 & 43 & 42 & 36 & 78 & 42 & 51 & 67 & 52 & 22 \\
\hline 17 & 27 & 14 & 42 & 43 & 34 & 77 & 40 & 54 & 61 & 50 & 18 \\
\hline 18 & 38 & 12 & 40 & 36 & 28 & 64 & 39 & 62 & 64 & 44 & 13 \\
\hline 19 & 26 & 17 & 46 & 48 & 37 & 85 & 44 & 59 & 65 & 59 & 22 \\
\hline 20 & 34 & 14 & 100 & 38 & 27 & 65 & 37 & 59 & 50 & 29 & 19 \\
\hline 21 & 29 & 16 & 46 & 42 & 31 & 73 & 39 & 60 & 60 & 52 & 14 \\
\hline 22 & 20 & 12 & 100 & 48 & 39 & 87 & 44 & 61 & 55 & 50 & 18 \\
\hline 23 & 22 & 13 & 47 & 39 & 37 & 76 & 43 & 67 & 64 & 50 & 17 \\
\hline 24 & 26 & 13 & 47 & 34 & 37 & 71 & 30 & 74 & 53 & 41 & 20 \\
\hline 25 & 31 & 15 & 100 & 35 & 28 & 63 & 40 & 72 & 60 & 50 & 13 \\
\hline 26 & 23 & 15 & 46 & 36 & 34 & 70 & 34 & 51 & 65 & 39 & 17 \\
\hline 27 & 23 & 15 & 100 & 36 & 37 & 73 & 40 & 63 & 50 & 47 & 14 \\
\hline 28 & 21 & 15 & 44 & 42 & 38 & 80 & 40 & 70 & 64 & 58 & 22 \\
\hline 29 & 22 & 16 & 46 & 27 & 36 & 63 & 42 & 73 & 56 & 55 & 23 \\
\hline 30 & 26 & 15 & 43 & 44 & 36 & 80 & 44 & 54 & 64 & 58 & 18 \\
\hline 31 & 23 & 15 & 43 & 37 & 38 & 75 & 43 & 63 & 46 & 48 & 20 \\
\hline 32 & 24 & 13 & 100 & 37 & 40 & 77 & 43 & 71 & 65 & 59 & 21 \\
\hline 33 & 30 & 14 & 45 & 43 & 32 & 75 & 42 & 60 & 61 & 51 & 17 \\
\hline 34 & 30 & 16 & 43 & 49 & 39 & 88 & 45 & 71 & 66 & 52 & 22 \\
\hline 35 & 34 & 17 & 44 & 41 & 31 & 72 & 44 & 61 & 63 & 33 & 18 \\
\hline 36 & 38 & 15 & 43 & 43 & 37 & 80 & 31 & 58 & 61 & 29 & 12 \\
\hline 37 & 26 & 19 & 100 & 48 & 37 & 85 & 40 & 64 & 76 & 59 & 19 \\
\hline & & & & & & & & & & & \\
\hline
\end{tabular}

Note: Values shown in bold are outliers which have been adjusted (see text). 


\begin{tabular}{|c|c|c|c|c|c|c|c|c|}
\hline $\mathbf{S}$ & FR5-FR1 & $\mathrm{CF} 1+\mathrm{CF}_{2}$ & CF4-CF1 & CFT $1+$ CFT2 & CFT4-CFT1 & WKMTOT & NOAC & IDENT \\
\hline 1 & 10 & 108 & 29 & 167 & 42 & 33 & 100 & 29 \\
\hline 2 & 5 & 105 & 29 & 240 & 64 & 39 & 100 & 0 \\
\hline 3 & 5 & 128 & 15 & 183 & 9 & 26 & 100 & 86 \\
\hline 4 & 4 & 124 & 17 & 193 & 17 & 36 & 88 & 75 \\
\hline 5 & 6 & 103 & 28 & 208 & -27 & 37 & 100 & 43 \\
\hline 6 & 0 & 138 & 5 & 165 & 26 & 31 & 100 & 57 \\
\hline 7 & 10 & 133 & 9 & 168 & 62 & 34 & 100 & 14 \\
\hline 8 & 8 & 109 & 22 & 172 & -15 & 34 & 82 & 29 \\
\hline 9 & 8 & 131 & 23 & 141 & 34 & 38 & 86 & $\overline{43}$ \\
\hline 11 & 8 & 113 & 24 & 131 & -16 & 37 & 100 & 100 \\
\hline 12 & 4 & 107 & 24 & 155 & 9 & 34 & 100 & 29 \\
\hline 13 & 5 & 105 & 26 & 194 & 12 & 38 & 100 & 71 \\
\hline 14 & 4 & 95 & 27 & 156 & -4 & 36 & 82 & 14 \\
\hline 15 & 7 & 126 & 14 & 200 & 26 & 39 & 100 & 86 \\
\hline 16 & 6 & 110 & 24 & 126 & -7 & 37 & 86 & 57 \\
\hline 17 & 8 & 98 & 21 & 116 & -8 & 39 & 100 & 38 \\
\hline 18 & 4 & 90 & 33 & 169 & 51 & 27 & 83 & 67 \\
\hline 19 & 6 & 135 & 5 & 150 & 37 & 37 & 100 & 88 \\
\hline 20 & 5 & 102 & 20 & 168 & 3 & 40 & 100 & 57 \\
\hline 21 & 9 & 100 & 34 & 126 & -11 & 40 & 88 & 50 \\
\hline 22 & 7 & 111 & 23 & 150 & 31 & 40 & 100 & 25 \\
\hline 23 & 6 & 115 & 17 & 128 & -6 & 38 & 100 & 25 \\
\hline 24 & 2 & 92 & 21 & 178 & 44 & 40 & 100 & 50 \\
\hline 25 & 7 & 131 & 0 & 100 & -5 & 26 & 100 & 63 \\
\hline 26 & 9 & 91 & 35 & 201 & 23 & 35 & 100 & 38 \\
\hline 27 & 6 & 99 & 26 & 240 & 18 & 40 & 100 & 63 \\
\hline 28 & 6 & 126 & 13 & 129 & 22 & 36 & 88 & 50 \\
\hline 29 & 4 & 127 & 5 & 100 & 2 & 40 & 100 & 100 \\
\hline 30 & 6 & 131 & 13 & 106 & 14 & 27 & 100 & 71 \\
\hline 31 & 5 & 105 & 32 & 129 & 32 & 31 & 100 & 88 \\
\hline 32 & 8 & 111 & 20 & 233 & 58 & 34 & 100 & 86 \\
\hline 33 & 8 & 116 & 19 & 133 & 11 & 39 & 100 & 63 \\
\hline 34 & 4 & 104 & 31 & 150 & -24 & 40 & 100 & 75 \\
\hline 35 & 6 & 115 & 25 & 131 & -15 & 38 & 100 & 50 \\
\hline 36 & 4 & 73 & 42 & 128 & -1 & 30 & 100 & 25 \\
\hline 37 & 5 & 104 & 26 & 96 & -13 & 34 & 100 & 88 \\
\hline
\end{tabular}

Note: Values shown in bold are outliers which have been adjusted (see text). 


\begin{tabular}{|c|c|c|c|c|c|c|c|c|c|c|}
\hline S & ALT & SP & HDG & POS & RATE & ACAD & SIM & AV.RATE & AV.OALL & AV.PICT \\
\hline 1 & 86 & 57 & 43 & $1 C 0$ & 15 & 97 & 74 & 121 & 5 & 4 \\
\hline 2 & 43 & 0 & 71 & 33 & 13 & 90 & 81 & 125 & 5 & 4 \\
\hline 3 & 57 & 0 & 100 & 100 & 14 & 85 & 86 & 142 & 5 & 5 \\
\hline 4 & 63 & 0 & 75 & 100 & 30 & 91 & 82 & 119 & 5 & 4 \\
\hline 5 & 29 & 14 & 86 & 100 & 10 & 86 & 76 & 85 & 2 & 3 \\
\hline 6 & 57 & 71 & 86 & 66 & 21 & 96 & 81 & 151 & 5 & 5 \\
\hline 7 & 100 & 29 & 86 & 100 & 18 & 87 & 87 & 154 & 6 & 6 \\
\hline 8 & 29 & 0 & 29 & 66 & 25 & 86 & 72 & 92 & 2 & 4 \\
\hline 9 & 86 & 29 & 43 & 100 & 37 & 90 & 81 & 137 & 5 & 5 \\
\hline 11 & 100 & 0 & 88 & 100 & 29 & 94 & 88 & 117 & 4 & 5 \\
\hline 12 & 29 & 29 & 85 & 100 & 18 & 88 & 72 & 131 & 5 & 4 \\
\hline 13 & 71 & 86 & 100 & 100 & 17 & 95 & 74 & 113 & 4 & 4 \\
\hline 14 & 43 & 14 & 71 & 66 & 15 & 91 & 74 & 82 & 1 & 2 \\
\hline 15 & 86 & 100 & 86 & 100 & 21 & 91 & 72 & 89 & 2 & 3 \\
\hline 16 & 57 & 0 & 57 & 66 & 27 & 83 & 83 & 150 & 5 & 5 \\
\hline 17 & 100 & 75 & 100 & 100 & 23 & 87 & 82 & 131 & 5 & 4 \\
\hline 18 & 50 & 17 & 50 & 66 & 22 & 89 & 80 & 124 & 4 & 4 \\
\hline 19 & 75 & 88 & 100 & 67 & 24 & 91 & 82 & 137 & 5 & 5 \\
\hline 20 & 71 & 0 & 86 & 100 & 19 & 98 & 66 & 75 & 1 & 2 \\
\hline 21 & 75 & 75 & 88 & 67 & 23 & 96 & 81 & 136 & 4 & 4 \\
\hline 22 & 100 & 75 & 100 & 100 & 23 & 88 & 89 & 135 & 4 & 4 \\
\hline 23 & 88 & 63 & 63 & 100 & 22 & 84 & 75 & 103 & 3 & 3 \\
\hline 24 & 50 & 0 & 88 & 67 & 38 & 94 & 79 & 117 & 4 & 4 \\
\hline 25 & 88 & 88 & 100 & 100 & 24 & 86 & 81 & 101 & 4 & 4 \\
\hline 26 & 100 & 63 & 100 & 100 & 23 & 89 & 77 & 126 & 4 & 4 \\
\hline 27 & 100 & 88 & 100 & 100 & 30 & 89 & 83 & 134 & 5 & 4 \\
\hline 28 & 88 & 75 & 38 & 33 & 25 & 85 & 73 & 112 & 3 & 4 \\
\hline 29 & 50 & 100 & 88 & 67 & 33 & 91 & 85 & 137 & 6 & 5 \\
\hline 30 & 100 & 86 & 100 & 100 & 23 & 86 & 92 & 163 & 7 & 6 \\
\hline 31 & 100 & 100 & 100 & 100 & 26 & 88 & 82 & 124 & 4 & 4 \\
\hline 32 & 86 & 100 & 100 & 100 & 26 & 97 & 80 & 136 & 6 & 5 \\
\hline 33 & 88 & 88 & 100 & 100 & 31 & 88 & 87 & 131 & 4 & 4 \\
\hline 34 & 50 & 100 & 75 & 31 & 22 & 93 & 71 & 114 & 3 & 4 \\
\hline 35 & 63 & 75 & 100 & 100 & 23 & 95 & 84 & 123 & 4 & 5 \\
\hline 36 & 100 & 63 & 100 & 100 & 20 & 86 & 62 & 84 & 2 & 2 \\
\hline 37 & 88 & 63 & 75 & 100 & 24 & 93 & 84 & 136 & 5 & 5 \\
\hline & & & & & & & & & 5 \\
\hline
\end{tabular}

Note: Values shown in bold are outliers which have been adjusted (see text). 


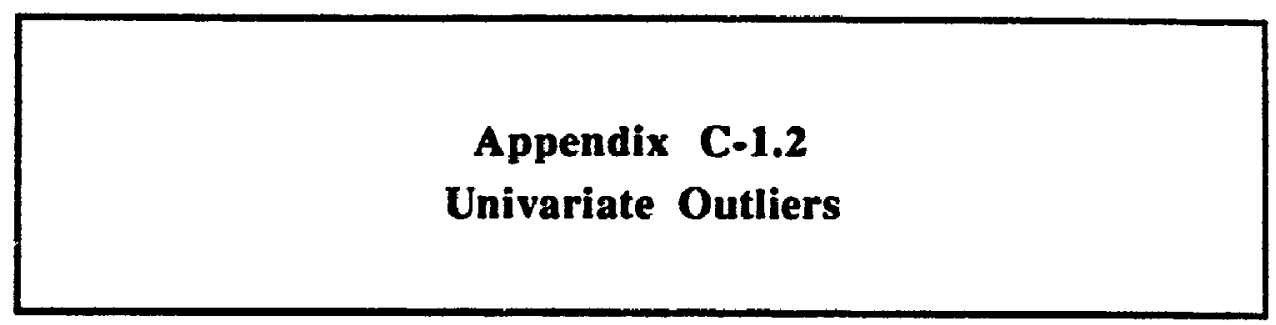




\begin{tabular}{|c|c|c|c|c|c|c|c|c|c|c|c|}
\hline $\mathbf{S}$ & AGE & EDUC. & MCAT & VR & NA & VR+NA & $\mathbf{A R}$ & CS\&A & MR & SR & FR1+FR2 \\
\hline 1 & 0.76 & -0.03 & -1.03 & 1.06 & -0.87 & 0.41 & -0.42 & 0.45 & 0.33 & -0.51 & -0.89 \\
\hline 2 & 1.17 & -0.03 & -0.16 & 1.06 & 1.01 & 1.24 & 0.91 & -0.32 & 0.48 & -0.06 & 0.14 \\
\hline 3 & -1.53 & -1.49 & $-1,89$ & -1.01 & -0.34 & -0.90 & 0.11 & 0.48 & -1.32 & 1.06 & 0.66 \\
\hline 4 & 0.55 & 1.43 & 0.70 & 0.74 & 0.74 & 0.88 & 0.38 & -1.25 & 0.93 & 0.84 & -0.89 \\
\hline 5 & -0.28 & 2.89 & -1.46 & 1.06 & -0.60 & 0.53 & 0.65 & -1.52 & -0.12 & 0.16 & 0.14 \\
\hline 6 & 0.13 & 0.46 & -2.32 & -0.53 & 1.01 & 0.05 & -0.42 & 1.27 & 2.13 & 0.84 & 2.98 \\
\hline 7 & -0.70 & -0.51 & -0.50 & -0.85 & -2.21 & -1.62 & -0.16 & 1.40 & -1.32 & -1.75 & -0.63 \\
\hline 8 & -0.28 & 0.95 & 1.13 & 1.06 & 1.01 & 1.24 & 0.91 & -1.25 & 0.03 & 0.95 & -1.92 \\
\hline 9 & -1.33 & 0.95 & 0.27 & -2.61 & -0.60 & -2.21 & -1.49 & -1.12 & -0.72 & -0.85 & -0.63 \\
\hline 11 & -0.08 & 0.95 & -0.59 & 1.22 & 0.74 & 1.24 & 1.18 & 0.08 & 0.03 & 0.61 & -0.63 \\
\hline 12 & 0.34 & -0.03 & -0.16 & -0.53 & -0.07 & -0.43 & -0.42 & -1.25 & 1.23 & 0.50 & 1.69 \\
\hline 13 & 0.55 & 0.46 & -0.16 & 0.43 & -0.07 & 0.29 & -0.16 & -1.25 & 0.48 & -1.19 & 0.66 \\
\hline 14 & -0.28 & -1.49 & & 1.22 & 1.27 & 1.48 & -0.16 & -0.98 & 1.08 & 1.18 & 0.40 \\
\hline 15 & 1.59 & -1.49 & 0.27 & 1.06 & 0.74 & 1.12 & 1.18 & -0.32 & 0.18 & 1.06 & 0.66 \\
\hline 16 & -0.28 & -0.51 & .0 .16 & 0.11 & 0.20 & 0.17 & 0.38 & -1.25 & 0.78 & 0.28 & 0.92 \\
\hline 17 & -0.08 & -0.51 & -0.59 & 0.27 & -0.34 & 0.05 & -0.16 & -0.85 & -0.12 & 0.05 & -0.11 \\
\hline 18 & 2.22 & -1.49 & -1.46 & -0.85 & -1.94 & -1.50 & -0.42 & 0.21 & 0.33 & -0.63 & -1.40 \\
\hline 19 & -0.28 & 0.95 & 1.13 & 1.06 & 0.47 & 1.00 & 0.91 & -0.19 & 0.48 & 1.06 & 0.92 \\
\hline 20 & 1.38 & -0.51 & & -0.53 & -2.21 & -1.38 & -0.96 & -0.19 & -1.77 & -2.32 & 0.14 \\
\hline 21 & 0.34 & 0.46 & 1.13 & 0.11 & -1.14 & -0.43 & -0.42 & -0.06 & -0.27 & 0.28 & -1.15 \\
\hline 22 & -1.53 & -1.49 & & 1.06 & 1.01 & 1.24 & 0.91 & 0.08 & -1.02 & 0.05 & -0.11 \\
\hline 23 & -1.12 & -1.00 & 1.56 & -0.37 & 0.47 & -0.07 & 0.65 & 0.87 & 0.33 & 0.05 & -0.37 \\
\hline 24 & -0.28 & -1.00 & 1.56 & -1.17 & 0.47 & -0.67 & -3.10 & 1.80 & -1.32 & -0.96 & 0.40 \\
\hline 25 & 0.76 & -0.03 & & -1.01 & -1.94 & -1.62 & -0.16 & 1.54 & -0.27 & 0.05 & -1.40 \\
\hline 26 & -0.91 & -0.03 & 1.13 & -0.85 & -0.34 & -0.78 & -1.76 & -1.25 & 0.48 & -1.19 & -0.37 \\
\hline 27 & -0.91 & -0.03 & & -0.85 & 0.47 & -0.43 & -0.16 & 0.34 & -1.77 & -0.29 & -1.15 \\
\hline 28 & -1.33 & -0.03 & 0.27 & 0.11 & 0.74 & 0.41 & -0.16 & 1.27 & 0.33 & 0.95 & 0.92 \\
\hline 29 & -1.12 & 0.46 & 1.13 & -2.29 & 0.20 & -1.62 & 0.38 & 1.67 & -0.87 & 0.61 & 1.17 \\
\hline 30 & -0.28 & $=0.03$ & -0.16 & 0.43 & 0.20 & 0.41 & 0.91 & -0.85 & 0.33 & 0.95 & -0.11 \\
\hline 31 & -0.91 & -0.03 & -0.16 & -0.69 & 0.74 & -0.19 & 0.65 & 0.34 & -2.37 & -0.18 & 0.40 \\
\hline 32 & -0.70 & -1.00 & & -0.69 & 1.27 & 0.05 & 0.65 & 1.40 & 0.48 & 1.06 & 0.66 \\
\hline 33 & 0.55 & -0.51 & 0.70 & 0.27 & -0.87 & -0.19 & 0.38 & -0.06 & -0.12 & 0.16 & -0.37 \\
\hline 34 & 0.55 & 0.46 & -0.16 & 1.22 & 1.01 & 1.36 & 1.18 & 1.40 & 0.63 & 0.28 & 0.92 \\
\hline 35 & 1.38 & 0.95 & 0.27 & -0.05 & -1.14 & -0.55 & 0.91 & 0.08 & 0.18 & -1.87 & -0.11 \\
\hline 36 & 2.22 & -0.03 & -0.16 & 0.27 & 0.47 & 0.41 & -2.56 & -0.32 & -0.12 & -2.32 & -1.66 \\
\hline 37 & -0.28 & 1.92 & & 1.06 & 0.47 & 1.00 & .0 .16 & 0.48 & 2.13 & 1.06 & 0.14 \\
\hline
\end{tabular}




\begin{tabular}{|c|c|c|c|c|c|c|c|c|}
\hline $\mathbf{S}$ & FRS-FRI & $\mathrm{CF} 1+\mathrm{CF} 2$ & CF4-CFI & CFT1+CFT2 & CFT4-CFTI & WKMTOT & NOAC & IDENT \\
\hline 1 & 1.87 & -0.23 & 0.79 & 0.25 & 1.14 & -0.60 & 0.49 & -1.02 \\
\hline 2 & -0.45 & -0.43 & 0.79 & 2.14 & 2.03 & 0.81 & 0.49 & -2.12 \\
\hline 3 & -0.45 & 1.10 & -0.70 & 0.66 & -0.19 & -2.24 & 0.49 & 1.17 \\
\hline 4 & -0.91 & 0.84 & -0.49 & 0.92 & 0.13 & 0.10 & -1.08 & 0.76 \\
\hline 5 & 0.01 & -0.56 & 0.68 & 1.31 & -1.65 & 0.34 & 0.49 & -0.47 \\
\hline 6 & -2.77 & 1.77 & -1.76 & 0.20 & 0.50 & -1.07 & 0.49 & 0.07 \\
\hline 7 & 1.87 & 1.43 & -1.34 & 0.28 & 1.95 & -0.36 & 0.49 & .1 .57 \\
\hline 8 & 0.94 & -0.16 & 0.04 & 0.38 & -1.16 & -0.36 & -3.08 & -1.02 \\
\hline 9 & 0.94 & 1.30 & 0.15 & -0.42 & 0.82 & 0.57 & -1.30 & -0.47 \\
\hline 11 & 0.94 & 0.11 & 026 & -0.68 & -1.20 & 0.34 & 0.49 & 1.72 \\
\hline 12 & -0.91 & -0.29 & 0.26 & -0.06 & -0.19 & -0.36 & 0.49 & -1.02 \\
\hline 13 & -0.45 & -0.43 & 0.47 & 0.95 & -0.07 & 0.57 & 0.49 & 0,02 \\
\hline 14 & -0.91 & -1.09 & 0.58 & -0.04 & -0.72 & 0.10 & -3.08 & -1.57 \\
\hline 15 & 0.48 & 0.97 & -0.81 & 1.10 & 0.50 & 0.81 & 0.49 & 1.17 \\
\hline 16 & 0.01 & -0.09 & 0.26 & -0.81 & -0.84 & 0.34 & -1.30 & 0.07 \\
\hline 17 & 0.94 & -0.89 & -0.06 & -1.07 & -0.88 & 0.81 & 0.49 & -0.68 \\
\hline 18 & -0.91 & -1.42 & 1.21 & 0.30 & 1.51 & -2.00 & -1.60 & 0.44 \\
\hline 19 & 0.01 & 1.57 & -1.76 & -0.19 & 0.94 & 0.34 & 0.49 & 1.24 \\
\hline 20 & -0.45 & -0.63 & -0.17 & 0.28 & -0.43 & 1.04 & 0.49 & 0.07 \\
\hline 21 & 1.40 & -0.76 & 1.32 & -0.81 & -1.00 & 1.04 & -1.08 & -0.20 \\
\hline 22 & 0.48 & -0.03 & 0.15 & -0.19 & 0.70 & 1.04 & 0.49 & -1.16 \\
\hline 23 & 0.01 & 0.24 & -0.49 & -0.76 & -0.80 & 0.57 & 0.49 & -1.16 \\
\hline 24 & -1.84 & -1.29 & -0.06 & 0.53 & 1.22 & 1.04 & 0.49 & .0 .20 \\
\hline 25 & 0.48 & 1.30 & -2.29 & -1.48 & -0.76 & -2.24 & 0.49 & 0.28 \\
\hline 26 & 1.40 & -1.36 & 1.42 & 1.13 & 0.37 & -0.13 & 0.49 & -0.68 \\
\hline 27 & 0.01 & -0.83 & 0.47 & 2.14 & 0.17 & 1.04 & 0.49 & 0.28 \\
\hline 28 & 0.01 & 0.97 & -0.91 & -0.73 & 0.33 & 0.10 & -1.08 & -0.20 \\
\hline 29 & -0.91 & 1.04 & -1.76 & -1.48 & -0.48 & 1.04 & 0.49 & 1.72 \\
\hline 30 & 0.01 & 1.30 & -0.91 & -1.33 & 0.01 & -2.00 & 0.49 & 0.62 \\
\hline 31 & -0.45 & -0.43 & 1.11 & -0.73 & 0.74 & -1.07 & 0.49 & 1.24 \\
\hline 32 & 0.94 & -0.03 & -0.17 & 1.96 & 1.79 & -0.36 & 0.49 & 1.17 \\
\hline 33 & 0.94 & 0.30 & -0.27 & -0.50 & -0.11 & 0.81 & 0.49 & 0.28 \\
\hline 34 & -0.91 & -0.49 & 1.00 & -0.19 & -1.53 & 1.04 & 0.49 & 0.76 \\
\hline 35 & 0.01 & 0.24 & 0.36 & -0.68 & -1.16 & 0.57 & 0.49 & -0.20 \\
\hline 36 & -0.91 & -2.55 & $\therefore 7$ & -0.76 & -0.60 & -1.30 & 0.49 & -1.16 \\
\hline 37 & -0.45 & -0.49 & 0.47 & -1.59 & -1.08 & -0.36 & 0.49 & 1.24 \\
\hline
\end{tabular}




\begin{tabular}{|c|c|c|c|c|c|c|c|c|c|c|}
\hline $\mathbf{S}$ & ALT & SP & HDG & POS & RATE & $\triangle \mathrm{ACAD}$ & SIM & AV.RATE & AV.OALL & AV.PICT \\
\hline 1 & 0.53 & 0.11 & -1.89 & 0.61 & -1.22 & 1.62 & -0.86 & -0.05 & 0.42 & -0.16 \\
\hline 2 & -1.31 & .1 .41 & -0.51 & -2.12 & -1.46 & -0.04 & 0.30 & 0.15 & 0.42 & -0.16 \\
\hline 3 & -0.69 & -1.41 & 0.87 & 0.61 & -1.30 & -1.02 & 1.01 & 0.92 & 0.65 & 0.55 \\
\hline 4 & -0.47 & -1.41 & -0.34 & 0.61 & 0.96 & 0.25 & 0.45 & -0.13 & 0.42 & -0.16 \\
\hline 5 & -1.92 & -1.03 & 0.18 & 0.61 & -2.00 & -0.98 & -0.58 & -1.69 & -1.69 & .1 .57 \\
\hline 6 & .0 .69 & 0.49 & 0.18 & -0.78 & -0.35 & 1.28 & 0.20 & 1.36 & 0.89 & 1.26 \\
\hline 7 & 1.14 & -0.65 & 0.18 & 0.61 & -0.84 & -0.77 & 1.21 & 1.49 & 1.36 & 1.61 \\
\hline 8 & -1.92 & -1.41 & -2.58 & -0.78 & 0.19 & -0.80 & -1.14 & -1.40 & -1.22 & -0.51 \\
\hline 9 & 0.53 & .0 .65 & -1.89 & 0.61 & 2.00 & 0.08 & 0.23 & 0.72 & 0.89 & 0.55 \\
\hline 11 & 1.14 & -1.41 & 0.26 & 0.61 & 0.83 & 0.88 & 1.26 & -0.24 & 0.18 & 0.90 \\
\hline 12 & -1.92 & -0.65 & 0.18 & 0.61 & -0.83 & $-0 . \overline{35}$ & -1.09 & 0.42 & 0.42 & 0.20 \\
\hline 13 & -0.08 & 0.87 & 0.87 & 0.61 & -0.99 & 1.08 & 0.76 & -0.42 & -0.29 & -0.51 \\
\hline 14 & -1.31 & -1.03 & -0.51 & -0.78 & -1.17 & 0.14 & $-0,88$ & -1.86 & -2.16 & -1.92 \\
\hline 15 & 0.53 & 1.25 & 0.18 & 0.61 & -0.30 & 0.30 & -1.11 & -1.50 & -1.69 & .0 .86 \\
\hline 16 & -0.69 & -1.41 & -1.20 & -0.78 & 0.53 & -2.79 & 0.50 & 1.29 & 0.89 & 0.90 \\
\hline 17 & 1.14 & 0.59 & 0.87 & 0.61 & -0.06 & -0.73 & 0.35 & 0.41 & 0.65 & -0.16 \\
\hline 18 & -1.00 & -0.96 & -1.55 & -0.78 & -0.26 & -0.17 & 0.15 & 0.08 & -0.29 & -0.16 \\
\hline 19 & 0.07 & 0.92 & 0.87 & -0.75 & 0.07 & 0.14 & 0.45 & 0.72 & 0.65 & 0.55 \\
\hline 20 & -0.08 & -1.41 & 0.18 & 0.61 & -0.60 & 1.80 & -1.99 & -2.17 & -1.93 & -2.27 \\
\hline 21 & 0.07 & 0.59 & 0.26 & -0.75 & -0.02 & 1.44 & 0.30 & 0.67 & 0.18 & 0.20 \\
\hline 22 & 1.14 & 0.59 & 0.87 & 0.61 & 0.01 & -0.44 & 1.41 & 0.61 & 0.18 & 0.20 \\
\hline 23 & 0.61 & 0.25 & -0.94 & 0.61 & -0.16 & .1 .37 & -0.66 & -0.87 & -0.99 & -0.86 \\
\hline 24 & $-1,00$ & -1.41 & 0.26 & -0.75 & 3.24 & 0.83 & -0.13 & -0.21 & -0.05 & -0.16 \\
\hline 25 & 0.61 & 0.92 & 0.87 & 0.61 & 0.16 & -0.86 & 0.28 & -0.98 & -0.29 & -0.16 \\
\hline 26 & 1.14 & 0.25 & 0.87 & 0.61 & -0.02 & -0.26 & -0.33 & 0.19 & -0.29 & -0.51 \\
\hline 27 & 1.14 & 0.92 & 0.87 & 0.61 & 0.94 & -0.13 & 0.58 & 0.58 & 0.42 & 0.20 \\
\hline 28 & 0.61 & 0.59 & -2.15 & -2.11 & 0.27 & -1.02 & -1.01 & -0.44 & -0.76 & -0.51 \\
\hline 29 & -1.00 & 1.25 & 0.26 & -0.75 & 1.42 & 0.30 & 0.88 & 0.70 & 1.12 & 0.90 \\
\hline 30 & 1.14 & 0.87 & 0.87 & 0.61 & -0.05 & -0.82 & 1.94 & 1.90 & 1.82 & 2.31 \\
\hline 31 & 1.14 & 1.25 & 0.87 & 0.61 & 0.33 & -0.48 & 0.33 & 0.10 & -0.05 & 0.20 \\
\hline 32 & 0.53 & 1.25 & 0.87 & 0.61 & 0.36 & 1.62 & 0.07 & 0.66 & 1.59 & 1.26 \\
\hline 33 & 0.61 & 0.92 & 0.87 & 0.61 & 1.13 & -0.48 & 1.21 & 0.41 & 0.18 & -0.16 \\
\hline 34 & -1.00 & 1.25 & -0.34 & -3.46 & -0.23 & 0.79 & -1.26 & -0.38 & -0.76 & -0.16 \\
\hline 35 & 0.47 & 0.59 & 0.87 & 0.61 & -0.09 & 1.21 & 0.65 & 0.07 & 0.18 & 0.55 \\
\hline 36 & 1.14 & 0.25 & 0.87 & 0.61 & -0.52 & -0.84 & -2.67 & -1.77 & -1.69 & -2.27 \\
\hline 37 & 0.61 & 0.25 & -0.34 & 0.61 & 0.05 & 0.59 & 0.70 & 0.66 & 0.65 & 0.90 \\
\hline
\end{tabular}


Appendix C.1.3

Skewness and Kurtosis of Variables 


\begin{tabular}{|l|c|c|c|c|c|c|c|c|c|}
\hline VARIABLE & $\mathrm{N}$ & $\mathrm{M}$ & $\mathrm{SD}$ & $\mathrm{MIN}$ & $\mathrm{MAX}$ & SKEWNESS & $\mathrm{Z}^{*}$ & KURTOSIS & $\mathrm{Z}^{*}$ \\
\hline AGE & 36 & 27.4 & 4.8 & 20.0 & 38.0 & 0.5 & 1.2 & -0.4 & -0.5 \\
\hline EDUC & 36 & 15.1 & 2.1 & 12.0 & 21.0 & 0.5 & 1.3 & 0.5 & 0.6 \\
\hline MCAT & 29 & 43.4 & 2.3 & 38.0 & 47.0 & -0.4 & -0.9 & -0.3 & -0.4 \\
\hline VR & 36 & 41.3 & 6.3 & 25.0 & 49.0 & -0.6 & -1.5 & -0.1 & -0.2 \\
\hline NA & 36 & 35.3 & 3.7 & 27.0 & 40.0 & -0.8 & -2.1 & -0.3 & -0.3 \\
\hline VR+NA & 36 & 76.6 & 8.4 & 58.0 & 89.0 & -0.4 & -1.0 & -0.7 & -0.9 \\
\hline AR & 36 & 40.6 & 3.7 & 30.0 & 45.0 & -1.2 & -3.0 & 1.4 & 1.7 \\
\hline CS\&A & 36 & 60.4 & 7.5 & 49.0 & 74.0 & 0.2 & 0.6 & -1.1 & -1.3 \\
\hline MR & 36 & 61.8 & 6.7 & 46.0 & 76.0 & -0.2 & -0.6 & 0.2 & 0.2 \\
\hline SR & 36 & 49.6 & 8.9 & 29.0 & 60.0 & -0.9 & -2.1 & -0.2 & -0.2 \\
\hline FRI+FR2 & 36 & 18.3 & 3.6 & 11.0 & 26.0 & -0.1 & -0.1 & -0.5 & -0.6 \\
\hline FR5-FRI & 36 & 6.0 & 2.2 & 0.0 & 10.0 & -0.3 & -0.7 & 0.3 & 0.4 \\
\hline CF1+CF2 & 36 & 111.4 & 15.0 & 73.0 & 138.0 & -0.1 & -0.3 & -0.4 & -0.4 \\
\hline CF4-CF1 & 36 & 21.6 & 9.4 & 0.0 & 42.0 & -0.3 & -0.9 & -0.1 & -0.2 \\
\hline CFT1+CFT2 & 36 & 157.4 & 38.7 & 96.0 & 240.0 & 0.5 & 1.2 & -0.4 & -0.5 \\
\hline CFT4-CFT! & 36 & 13.8 & 24.7 & -27.0 & 64.0 & 0.4 & 0.9 & -0.8 & -0.9 \\
\hline WKMTOT & 36 & 35.6 & 4.3 & 26.0 & 40.0 & -0.9 & -2.3 & -0.1 & -0.1 \\
\hline NOAC & 36 & 96.7 & 6.3 & 82.3 & 100.0 & -1.4 & -3.5 & 0.2 & 0.2 \\
\hline IDENT & 36 & 55.2 & 26.1 & 0.0 & 100.0 & -0.1 & -0.3 & -0.9 & -1.1 \\
\hline ALT & 36 & 73.4 & 23.4 & 28.6 & 100.0 & -0.5 & -1.1 & -1.0 & -1.2 \\
\hline SP & 36 & 52.9 & 37.7 & 0.0 & 100.0 & -0.3 & -0.8 & -1.5 & -1.8 \\
\hline HDG & 36 & 82.0 & 20.7 & 28.6 & 100.0 & -1.1 & -2.7 & 0.2 & 0.2 \\
\hline POS & 36 & 85.9 & 21.9 & 31.0 & 100.0 & -1.3 & -3.1 & 0.4 & 0.5 \\
\hline RATE & 36 & 23.2 & 6.3 & 9.6 & 38.2 & 0.3 & 0.7 & 0.2 & 0.3 \\
\hline ACAD & 36 & 90.1 & 4.1 & 82.8 & 98.0 & 0.3 & 0.7 & -0.9 & -1.1 \\
\hline SIM & 36 & 79.3 & 6.6 & 61.7 & 92.2 & -0.5 & -1.3 & 0.1 & 0.1 \\
\hline AVG.RATE & 36 & 121.8 & 21.6 & 75.0 & 163.0 & -0.5 & -1.2 & -0.4 & -0.5 \\
\hline AVG.0ALL & 36 & 4.1 & 1.4 & 1.0 & 6.7 & -0.5 & -1.3 & -0.4 & -0.5 \\
\hline AVG.PICT & 36 & 4.1 & 0.9 & 2.0 & 6.3 & -0.4 & -0.9 & 0.5 & 0.6 \\
\hline & & & & & & & & & \\
\hline
\end{tabular}

* If $Z$ exceeds the critical value of $+/-2.86$ the variable is considered to be extremely skewed or kurtosed (Tabachnick and Fidell, 1989) 


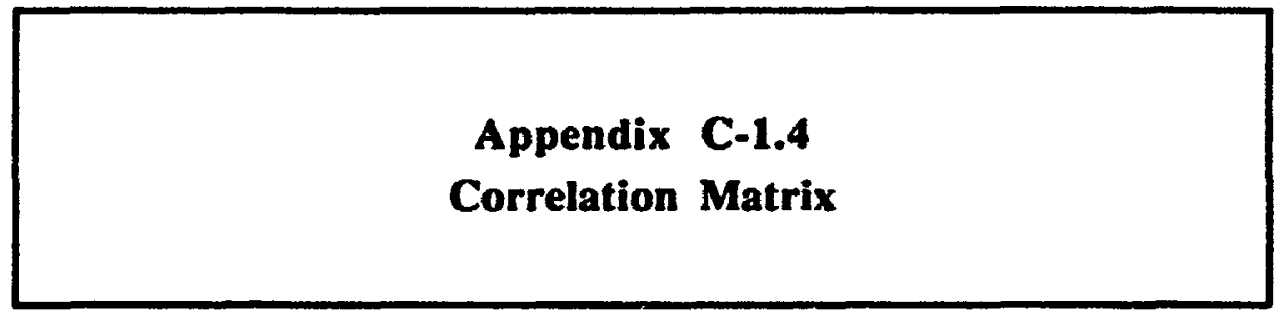




\begin{tabular}{|c|c|c|c|c|c|c|c|c|c|c|c|}
\hline & $A C E$ & EDUC & MCAT & VR & $\mathbf{M}$ & $\sqrt{R+N A}$ & $\mathbf{A R}$ & CSRA & $\mathbf{M R}$ & SR & $\overline{F R 1+F R 2}$ \\
\hline AGE & 1.00 & & & & & & & & & & \\
\hline EDUC & 0.00 & 1.00 & & & & & & & & & \\
\hline MCAT & -0.17 & 0.02 & 1.00 & & & & & & & & \\
\hline VR & 0.29 & 0.19 & -0.08 & 1.00 & & & & & & & \\
\hline NA & -0.30 & 0.02 & 0.20 & 0.37 & 1.00 & & & & & & \\
\hline $\mathrm{VR}+\mathrm{NA}$ & 0.08 & 0.15 & 0.02 & 0.91 & 0.72 & 1.00 & & & & & \\
\hline $\mathbf{A R}$ & -0.10 & 0.12 & -0.09 & 0.47 & 0.27 & 0.47 & 1.00 & & & & \\
\hline CS\&A & -0.16 & -0.25 & 0.03 & -0.35 & -0.01 & -0.27 & -0.03 & 1.00 & & & \\
\hline MR & 0.24 & 0.26 & -0.15 & 0.43 & 0.31 & 0.46 & 0.14 & -0.21 & 1.00 & & \\
\hline SR & -0.36 & 0.05 & -0.03 & 0.31 & 0.57 & 0.48 & 0.55 & 0.03 & 0.41 & 1.00 & \\
\hline$\overline{F R 1}+F R 2$ & .0 .25 & -0.07 & -0.18 & -0.04 & 0.35 & 0.12 & 0.18 & 0.18 & 0.26 & 0.34 & 1.00 \\
\hline FRS-FRI & -0.18 & 0.00 & 0.20 & 0.09 & .0 .34 & -0.08 & 0.17 & -0.21 & -0.22 & -0.10 & -0.48 \\
\hline CF1+CF2 & -0.34 & 0.11 & -0.08 & -0.17 & -0.03 & -0.14 & 0.44 & 0.24 & 0.06 & 0.41 & 0.31 \\
\hline CF4-CF1 & 0.28 & 0.05 & -0.03 & 0.24 & 0.07 & 0.21 & -0.27 & -0.41 & -0.01 & -0.37 & -0.32 \\
\hline CFT1+CFT2 & 0.06 & -0.10 & .0 .19 & 0.08 & 0.17 & 0.13 & 0.00 & .0 .16 & -0.08 & -0.06 & 0.02 \\
\hline CFT4-CFTI & -0.04 & -0.41 & -0.08 & -0.27 & -0.02 & -0.21 & -0.17 & 0.22 & -0.17 & -0.13 & 0.07 \\
\hline WKMTor & -0.11 & 0.11 & 0.57 & 0.15 & 0.19 & 0.20 & 0.10 & .0 .06 & -0.12 & -0.11 & 0.18 \\
\hline NOAC & 0.01 & 0.02 & -0.13 & -0.01 & -0.03 & -0.02 & 0.06 & 0.32 & -0.18 & -0.20 & 0.28 \\
\hline DENT & .0 .07 & 0.17 & -0.03 & -0.11 & 0.11 & -0.04 & 0.29 & 0.27 & -0.05 & 0.36 & 0.26 \\
\hline ALT & 0.18 & .0 .18 & 0.10 & -0.08 & -0.09 & .0 .10 & -0.09 & 0.16 & -0.27 & -0.21 & -0.29 \\
\hline SP & -0.07 & -0.03 & 0.18 & -0.05 & 0.12 & 0.02 & 0.21 & 0.34 & 0.01 & 0.11 & 0.17 \\
\hline HDG & 0.02 & -0.08 & -0.03 & -0.07 & -0.05 & -0.08 & 0.01 & 0.13 & -0.24 & -0.14 & 0.18 \\
\hline POS & -0.05 & 0.02 & -0.10 & -0.16 & -0.31 & -0.26 & .0 .10 & -0.26 & -0.24 & -0.26 & -0.27 \\
\hline RATE & -0.28 & 0.02 & 0.56 & -0.45 & 0.14 & -0.27 & -0.20 & 0.27 & -0.23 & 0.05 & -0.11 \\
\hline$\overline{\mathrm{ACAD}}$ & 0.31 & $\overline{0.13}$ & .0 .01 & 0.05 & -0.03 & 0.03 & -0.09 & 0.17 & 0.12 & .0 .14 & 0.15 \\
\hline SIM & -0.36 & -0.01 & .0 .08 & -0.19 & -0.22 & -0.24 & 0.33 & 0.16 & -0.24 & 0.18 & 0.03 \\
\hline AVG.RATE & .0 .40 & -0.02 & -0.17 & -0.30 & -0.01 & -0.23 & 0.12 & 0.18 & 0.07 & 0.20 & 0.25 \\
\hline AVG.OAL & .0 .35 & 0.05 & -0.13 & -0.33 & -0.02 & -0.26 & 0.13 & 0.20 & 0.05 & 0.19 & 0.18 \\
\hline AVG.PICT & -0.38 & 0.08 & -0.15 & -0.23 & 0.05 & -0.15 & 0.31 & 0.28 & 0.10 & 0.32 & 0.22 \\
\hline
\end{tabular}




\begin{tabular}{|c|c|c|c|c|c|c|c|c|}
\hline & FRS-FRI & CF1+CF2 & CF4-CF1 & CFT1+CFT2 & CFTA-CFII & WkMrTor & NOAC & DDENT \\
\hline FR5-FRI & 1.00 & & & & & & & \\
\hline CF1+CF2 & 0.08 & 1.00 & & & & & & \\
\hline CF4-CF1 & 0.08 & -0.85 & 1.00 & & & & & \\
\hline CFT1+CFT2 & .0 .01 & -0.17 & 0.20 & 1.00 & & & & \\
\hline CFT4-CFT1 & 0.06 & 0.16 & -0.12 & 0.46 & 1.00 & & & \\
\hline WKMTOT & 0.11 & -0.14 & 0.12 & 0.17 & -0.13 & 1.00 & & \\
\hline NOAC & -0.02 & 0.07 & -0.16 & 0.07 & 0.07 & 0.04 & 1.00 & \\
\hline IDENT & .0 .16 & 0.31 & .0 .29 & -0.16 & .0 .14 & -0.11 & 0.18 & 1.00 \\
\hline ALT & 0.48 & 0.07 & -0.03 & .0 .24 & 0.18 & -0.06 & 0.33 & 0.14 \\
\hline SP & 0.12 & 0.17 & -0.17 & -0.24 & 0.00 & 0.05 & 0.41 & $\mathbf{0 . 3 3}$ \\
\hline $\mathrm{HDG}$ & -0.08 & -0.03 & -0.10 & 0.03 & .0 .04 & -0.01 & 0.69 & 0.29 \\
\hline POS & 0.34 & 0.02 & 0.00 & -0.02 & -0.07 & .0 .19 & 0.38 & 0.11 \\
\hline RATE & -0.01 & 0.15 & -0.19 & -0.26 & 0.07 & 0.27 & -0.17 & 0.37 \\
\hline ACAD & .0 .09 & -0.08 & 0.11 & 0.20 & 0,13 & 0.29 & 0.16 & 0.25 \\
\hline SIM & 0.18 & 0.47 & -0.39 & -0.17 & 0.17 & -0.03 & 0.16 & 0.33 \\
\hline AVGRATE & 0.09 & 0.42 & .0 .25 & .0 .14 & 0.36 & -0.14 & 0.12 & 0.20 \\
\hline AVG.OAL & 0.14 & 0.46 & -0.33 & .0 .11 & 0.39 & -0.15 & 0.19 & 0.26 \\
\hline AVG.PICT & 0.12 & 0.57 & -0.40 & .0 .19 & 0.27 & -0.21 & 0.14 & 0.36 \\
\hline
\end{tabular}

\begin{tabular}{|l|c|c|c|c|c|c|c|c|c|c|}
\cline { 2 - 12 } \multicolumn{1}{c|}{} & NLT & SP & HDG & POS & RATE & ACAD & SIM & AV.RATE & AV.OALL & AV.PICT \\
\hline ALT & 1.00 & & & & & & & & & \\
\hline SP & 0.48 & 1.00 & & & & & & & & \\
\hline POS & 0.34 & 0.42 & 1.00 & & & & & & & \\
\hline RASTE & 0.48 & 0.09 & 0.44 & 1.00 & & & & & & \\
\hline ACAD & 0.25 & 0.13 & -0.04 & -0.01 & 1.00 & & & & & \\
\hline SIM & -0.06 & 0.12 & 0.10 & -0.03 & 0.06 & 1.00 & & & & \\
\hline AVG.RATE & 0.27 & 0.00 & 0.33 & 0.21 & 0.30 & -0.01 & 1.00 & & & \\
\hline AVG.OAL & 0.23 & 0.19 & 0.17 & 0.00 & 0.27 & -0.04 & 0.73 & 1.00 & & \\
\hline AVG.PICT & 0.20 & 0.21 & 0.19 & 0.10 & 0.32 & 0.06 & 0.73 & 0.94 & 1.00 & \\
\hline
\end{tabular}


Appendix C-1.5

ANOVA Data 


\section{C-1.5.A: ANOVA Data for Accuracy of Recall}

\begin{tabular}{|l|c|c|c|c|c|}
\hline \multicolumn{1}{|c|}{ TEST } & $\begin{array}{c}\text { SUM OF } \\
\text { SOUARES }\end{array}$ & MEAN SQUARE & of & F & $\begin{array}{c}\text { SIGN- } \\
\text { FICANCE }\end{array}$ \\
\hline BETWEEN GROUPS & 33156.37 & 8289.09 & 4 & 11.68 & $\mathbf{p}<.000$ \\
\hline WITHIN GROUPS & 124534.36 & 711.62 & 175 & & \\
\hline TOTAL & 157690.73 & & 179 & & \\
\hline
\end{tabular}

\section{C-1.5.B: Scheffé Test Data}

\begin{tabular}{|l|c|c|c|c|}
\hline \multicolumn{1}{|c|}{ TEST } & MEAN A & MEAN B & SCHEFFE F & SIGN- \\
FICANCE \\
\hline IDENT VS. SPEED & 55.28 & 52.97 & 0.034 & NS \\
\hline IDENT VS HEADING & 55.28 & 82.14 & 4.54 & $\mathrm{p}<.05$ \\
\hline IDENT VS. POSITION & 55.28 & 85.97 & 5.96 & $\mathrm{p}<.05$ \\
\hline IDENT VS.ALTITU.DE & 55.28 & 73.44 & 2.09 & NS \\
\hline SPEED VS. HEADING & 52.97 & 82.14 & 5.38 & $\mathrm{p}<.05$ \\
\hline SPEED VS. POSITION & 52.97 & 85.97 & 6.89 & $\mathrm{p}<.05$ \\
\hline SPEED VS ALTITUDE & 52.97 & 73.44 & 2.65 & $\mathrm{p}<.05$ \\
\hline HEADING VS. POSITION & 82.14 & 85.97 & 0.09 & NS \\
\hline HEADING VS. ALTITUDE & 82.14 & 73.44 & 0.48 & NS \\
\hline POSITION VS. ALTITUDE & 82.14 & 73.44 & 0.99 & NS \\
\hline
\end{tabular}




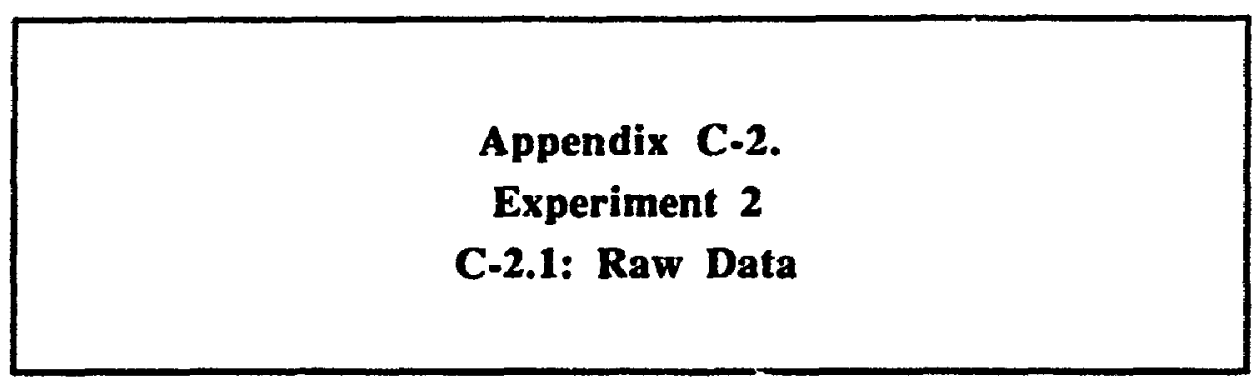




\begin{tabular}{|c|c|c|c|c|c|c|c|c|c|c|c|}
\hline S & AGE & EDUC & MCAT & VR & NA & VR+NA & AR & CS\&A & MR & SR & FR1+FR2 \\
\hline 1 & 30 & 12 & 45 & 35 & 37 & 72 & 41 & 75 & 62 & 47 & 16 \\
\hline 2 & 24 & 17 & 46 & 43 & 35 & 78 & 41 & 53 & 68 & 55 & 25 \\
\hline 3 & 25 & 16 & 47 & 43 & 34 & 77 & 44 & 72 & 67 & 51 & 21 \\
\hline 4 & 29 & 16 & 41 & 40 & 23 & 63 & 38 & 65 & 59 & 55 & 18 \\
\hline 5 & 26 & 14 & 46 & 43 & 36 & 79 & 41 & 78 & 56 & 52 & 17 \\
\hline 6 & 25 & 16 & 49 & 47 & 38 & 85 & 42 & 73 & 64 & 59 & 15 \\
\hline 7 & 22 & 17 & 46 & 42 & 39 & 81 & 45 & 65 & 54 & 52 & 29 \\
\hline 8 & 32 & 18 & 42 & 48 & 34 & 82 & 43 & 62 & 66 & 51 & 19 \\
\hline 9 & 23 & 14 & 42 & 35 & 37 & 72 & 39 & 58 & 61 & 44 & 13 \\
\hline 10 & 23 & 14 & 49 & 42 & 29 & 71 & 36 & 58 & 65 & 55 & 17 \\
\hline 11 & 30 & 12 & 44 & 47 & 28 & 75 & 43 & 60 & 59 & 54 & 18 \\
\hline 12 & 20 & 14 & 45 & 43 & 32 & 75 & 36 & 73 & 66 & 54 & 29 \\
\hline 13 & 27 & 16 & 41 & 47 & 33 & 80 & 44 & 61 & 59 & 48 & .26 \\
\hline 14 & 26 & 17 & 46 & 47 & 33 & 80 & 39 & 69 & 64 & 51 & 21 \\
\hline 15 & 24 & 12 & 46 & 48 & 36 & 84 & 39 & 79 & 65 & 49 & 18 \\
\hline 16 & 30 & 19 & 37 & 45 & 36 & 81 & 43 & 72 & 64 & 43 & 19 \\
\hline 17 & 24 & 12 & 43 & 48 & 33 & 81 & 44 & 60 & 63 & 57 & 26 \\
\hline
\end{tabular}

Note: Values shown in bold are outliers which have been adjusted (see text).

\begin{tabular}{|c|c|c|c|c|c|c|c|c|}
\hline S & FR5-FR1 & CF1+CF2 & CF4-CF1 & CFT1+CFT2 & CFT4-CFT1 & WKMTOT & NOAC & IDENT \\
\hline 1 & 9 & 112 & 23 & 109 & -14 & 40 & 75 & 50 \\
\hline 2 & 5 & 120 & 14 & 126 & 14 & 40 & 88 & 0 \\
\hline 3 & 8 & 121 & 14 & 136 & 13 & 35 & 80 & 50 \\
\hline 4 & 6 & 82 & 36 & 134 & -23 & 31 & 80 & 25 \\
\hline 5 & 6 & 108 & 30 & 157 & -3 & 35 & 88 & 38 \\
\hline 6 & 4 & 103 & 23 & 240 & -33 & 40 & 100 & 0 \\
\hline 7 & 1 & 136 & 3 & 228 & -53 & 40 & 75 & 63 \\
\hline 8 & 7 & 120 & 18 & 106 & -16 & 40 & 100 & 0 \\
\hline 9 & 5 & 104 & 18 & 125 & -14 & 38 & 75 & 13 \\
\hline 10 & 5 & 113 & 20 & 178 & -9 & 35 & 100 & 25 \\
\hline 11 & 8 & 107 & 30 & 240 & -49 & 40 & 100 & 0 \\
\hline 12 & 1 & 121 & 21 & 148 & -50 & 40 & 100 & 50 \\
\hline 13 & 4 & 109 & 23 & 147 & 19 & 36 & 100 & 38 \\
\hline 14 & 5 & 116 & 15 & 164 & 18 & 40 & 100 & 75 \\
\hline 15 & 5 & 107 & 23 & 136 & -9 & 36 & 100 & 0 \\
\hline 16 & 4 & 110 & 7 & 158 & -11 & 39 & 63 & 0 \\
\hline 17 & 2 & 105 & 31 & 153 & 13 & 37 & 100 & 63 \\
\hline
\end{tabular}




\begin{tabular}{|c|c|c|c|c|c|c|c|c|c|c|}
\hline S & ALT & SP & HDG & POS & RATE & ACAD & SIM & AV.RATE & AV.OALL & AV.PICT \\
\hline 1 & 50 & 50 & 88 & 57 & 9 & 92 & 79 & 117 & 4 & 4 \\
\hline 2 & 63 & 0 & 75 & 50 & 3 & 83 & 73 & 78 & 1 & 2 \\
\hline 3 & 100 & 88 & 100 & 71 & 4 & 85 & 76 & 107 & 4 & 4 \\
\hline 4 & 88 & 0 & 88 & 25 & 6 & 89 & 81 & 129 & 5 & 4 \\
\hline 5 & 88 & 38 & 75 & 38 & 9 & 95 & 76 & 115 & 4 & 3 \\
\hline 6 & 75 & 50 & 100 & 88 & 8 & 91 & 72 & 89 & 2 & 2 \\
\hline 7 & 88 & 38 & 88 & 57 & 6 & 81 & 66 & 69 & 1 & 1 \\
\hline 8 & 100 & 0 & 80 & 40 & 5 & 91 & 66 & 102 & 3 & 2 \\
\hline 9 & 88 & 0 & 100 & 50 & 11 & 90 & 73 & 114 & 4 & 3 \\
\hline 10 & 63 & 0 & 100 & 38 & 5 & 94 & 87 & 142 & 6 & 5 \\
\hline 11 & 63 & 0 & 100 & 75 & 5 & 82 & 79 & 108 & 3 & 3 \\
\hline 12 & 75 & 75 & 100 & 50 & 10 & 87 & 84 & 111 & 4 & 4 \\
\hline 13 & 75 & 100 & 100 & 50 & 6 & 93 & 83 & 147 & 6 & 5 \\
\hline 14 & 88 & 75 & 100 & 75 & & 95 & 83 & 128 & 5 & 5 \\
\hline 15 & 88 & 25 & 100 & 75 & 10 & 97 & 86 & 139 & 6 & 5 \\
\hline 16 & 61.5 & 0 & 63 & 25 & 7 & 85 & 84 & 119 & 5 & 4 \\
\hline 17 & 63 & 50 & 100 & 50 & 5 & 92 & 80 & 114 & 4 & 4 \\
\hline
\end{tabular}

Note: Values shown in bold are outliers which have been adjusted (see text). 


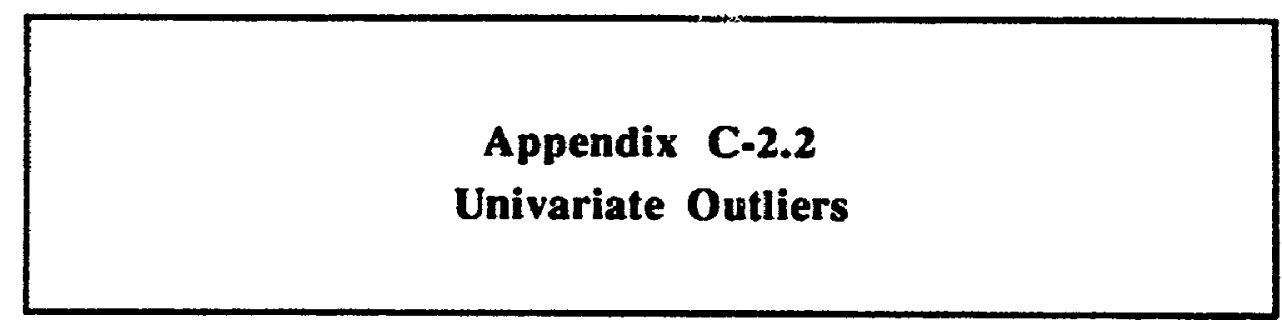




\begin{tabular}{|c|c|c|c|c|c|c|c|c|c|c|c|}
\hline S & AGE & EDUC & MCAT & VR & NA & VR+NA & AR & CS\&A & MR & SR & FR1+FR2 \\
\hline 1 & 1.24 & -1.36 & 0.19 & -2.11 & 0.82 & -0.97 & -0.02 & 1.07 & -0.12 & -1.05 & -0.91 \\
\hline 2 & -0.56 & 0.89 & 0.51 & -0.17 & 0.32 & 0.11 & -0.02 & -1.75 & 1.40 & 0.78 & 0.94 \\
\hline 3 & -0.26 & 0.44 & 0.83 & -0.17 & 0.07 & -0.07 & 1.04 & 0.68 & 1.15 & -0.13 & 0.12 \\
\hline 4 & 0.94 & 0.44 & -1.10 & -0.90 & -2.66 & -2.59 & -1.09 & -0.21 & -0.88 & 0.78 & -0.49 \\
\hline 5 & 0.04 & -0.46 & 0.51 & -0.17 & 0.57 & 0.29 & -0.02 & 1.45 & -1.64 & 0.09 & -0.70 \\
\hline 6 & -0.26 & 0.44 & 1.48 & 0.80 & 1.07 & 1.36 & 0.33 & 0.81 & 0.39 & 1.70 & -1.11 \\
\hline 7 & -1.16 & 0.66 & 0.51 & -0.41 & 1.31 & 0.64 & 1.40 & -0.21 & -2.15 & 0.09 & 1.76 \\
\hline 8 & 1.84 & 1.33 & -0.78 & 1.04 & 0.07 & 0.82 & 0.69 & -0.59 & 0.90 & -0.13 & -0.29 \\
\hline 9 & -0.86 & -0.46 & -0.78 & -2.11 & 0.82 & -0.97 & -0.73 & -1.11 & -0.37 & -1.74 & -1.52 \\
\hline 10 & -0.86 & -0.46 & 1.48 & -0.41 & -1.17 & -1.15 & -1.80 & -1.11 & 0.64 & 0.78 & -0.70 \\
\hline 11 & 1.24 & -1.36 & -0.13 & 0.80 & -1.42 & -0.43 & 0.69 & -0.85 & -0.88 & 0.55 & -0.49 \\
\hline 12 & -1.76 & -0.46 & 0.19 & -0.17 & -0.42 & -0.43 & -1.80 & 0.81 & 0.90 & 0.55 & 1.76 \\
\hline 13 & 0.34 & 0.44 & -1.10 & 0.80 & -0.18 & 0.46 & 1.04 & -0.72 & -0.88 & -0.82 & 1.15 \\
\hline 14 & 0.04 & 0.89 & 0.51 & 0.80 & -0.18 & 0.46 & -0.73 & 0.30 & 0.39 & -0.13 & 0.12 \\
\hline 15 & -0.56 & -1.36 & 0.51 & 1.04 & 0.57 & 1.18 & -0.73 & 1.58 & 0.64 & -0.59 & -0.49 \\
\hline 16 & 1.24 & 1.78 & -2.39 & 0.31 & 0.57 & 0.64 & 0.69 & 0.68 & 0.39 & -1.97 & -0.29 \\
\hline 17 & -0.56 & -1.36 & -0.45 & 1.04 & -0.18 & 0.64 & 1.04 & -0.85 & 0.13 & 1.24 & 1.15 \\
\hline
\end{tabular}

\begin{tabular}{|c|c|c|c|c|c|c|c|c|}
\hline $\mathrm{S}$ & FR5-FR1 & CF1+CF2 & CF4-CF1 & CFT1+CFT2 & CFT4-CFT1 & WKMTOT & NOAC & IDENT \\
\hline 1 & 1.75 & 0.05 & 0.29 & -1.17 & -0.08 & 0.83 & -1.16 & 0.81 \\
\hline 2 & 0.00 & 0.76 & -0.76 & -0.77 & 1.11 & 0.83 & -0.16 & -1.09 \\
\hline 3 & 1.31 & 0.85 & -0.76 & -0.53 & 1.06 & -1.02 & -0.76 & 0.81 \\
\hline 4 & 0.44 & -2.60 & 1.81 & -0.57 & -0.46 & -2.50 & -0.76 & -0.14 \\
\hline 5 & 0.44 & -0.30 & 1.11 & -0.02 & 0.39 & -1.02 & -0.16 & 0.33 \\
\hline 6 & -0.44 & -0.74 & 0.29 & 1.97 & -0.88 & 0.83 & 0.83 & -1.09 \\
\hline 7 & -1.75 & 2.17 & -2.05 & 1.68 & -1.72 & 0.83 & -1.16 & 1.28 \\
\hline 8 & 0.8 & 0.76 & -0.30 & -1.25 & -0.16 & 0.83 & 0.83 & -1.09 \\
\hline 9 & 0.00 & -0.65 & -0.30 & -0.79 & -0.08 & 0.09 & -1.16 & -0.61 \\
\hline 10 & 0.00 & 0.14 & -0.06 & 0.48 & 0.13 & -1.02 & 0.83 & -0.14 \\
\hline 11 & 1.31 & -0.39 & 1.11 & 1.97 & -1.56 & 0.83 & 0.83 & -1.09 \\
\hline 12 & -1.75 & 0.85 & 0.05 & -0.24 & -1.60 & 0.83 & 0.83 & 0.81 \\
\hline 13 & -0.44 & -0.21 & 0.29 & -0.26 & 1.32 & -0.65 & 0.83 & 0.33 \\
\hline 14 & 0.00 & 0.41 & -0.65 & 0.15 & 1.27 & 0.83 & 0.83 & 1.75 \\
\hline 15 & 0.00 & -0.39 & 0.29 & -0.53 & 0.13 & -0.65 & 0.83 & -1.09 \\
\hline 16 & -0.44 & -0.12 & -1.58 & 0.00 & 0.05 & 0.46 & -2.15 & -1.09 \\
\hline 17 & -1.31 & -0.57 & 1.22 & -0.12 & 1.06 & -0.28 & 0.83 & 1.28 \\
\hline
\end{tabular}




\begin{tabular}{|c|c|c|c|c|c|c|c|c|c|c|}
\hline S & ALT & SP & HDG & POS & RATE & ACAD & SIM & AV.RATE & AV.OALL & AV.PICT \\
\hline 1 & -1.82 & 0.44 & -0.33 & 0.19 & 0.77 & 0.52 & 0.07 & 0.17 & -0.01 & 0.12 \\
\hline 2 & -0.98 & -0.99 & -1.38 & -0.20 & -1.35 & -1.37 & -0.78 & -1.67 & -1.79 & -1.60 \\
\hline 3 & 1.54 & 1.51 & 0.71 & 0.98 & -1.16 & -0.84 & -0.27 & -0.29 & -0.23 & 0.12 \\
\hline 4 & 0.70 & -0.99 & -0.33 & -1.58 & -0.48 & -0.11 & 0.44 & 0.72 & 0.65 & 0.69 \\
\hline 5 & 0.70 & 0.08 & -1.38 & -0.89 & 0.85 & 1.19 & -0.39 & 0.09 & -0.01 & -0.17 \\
\hline 6 & -0.14 & 0.44 & 0.71 & 1.86 & 0.37 & 0.36 & -0.89 & -1.16 & -1.34 & -1.32 \\
\hline 7 & 0.70 & 0.08 & -0.33 & 0.19 & -0.50 & -1.75 & -1.83 & -2.11 & -1.79 & -1.89 \\
\hline 8 & 1.54 & -0.99 & -0.96 & -0.76 & -0.71 & 0.27 & -1.95 & -0.56 & -0.90 & -1.03 \\
\hline 9 & 0.70 & -0.99 & 0.71 & -0.20 & 1.87 & 0.09 & -0.81 & 0.03 & 0.21 & -0.17 \\
\hline 10 & -0.98 & -0.99 & 0.71 & -0.89 & -0.82 & 0.82 & 1.41 & 1.36 & 1.32 & 1.27 \\
\hline 11 & -0.98 & -0.99 & 0.71 & 1.17 & -0.79 & -1.55 & 0.19 & -0.24 & -0.46 & -0.74 \\
\hline 12 & -0.14 & 1.16 & 0.71 & -0.20 & 1.46 & -0.48 & 0.90 & -0.12 & 0.21 & 0.69 \\
\hline 13 & -0.14 & 1.87 & 0.71 & -0.20 & -0.37 & 0.62 & 0.81 & 1.58 & 1.32 & 1.27 \\
\hline 14 & 0.70 & 1.16 & 0.71 & 1.17 & & 1.17 & 0.75 & 0.69 & 0.87 & 0.98 \\
\hline 15 & 0.70 & -0.27 & 0.71 & 1.17 & 1.31 & 1.45 & 1.20 & 1.23 & 1.32 & 0.98 \\
\hline 16 & -1.05 & -0.99 & -2.42 & -1.58 & 0.29 & -0.98 & 0.92 & 0.25 & 0.43 & 0.41 \\
\hline 17 & -0.98 & 0.44 & 0.71 & -0.20 & -0.74 & 0.60 & 0.21 & 0.04 & 0.21 & 0.41 \\
\hline
\end{tabular}




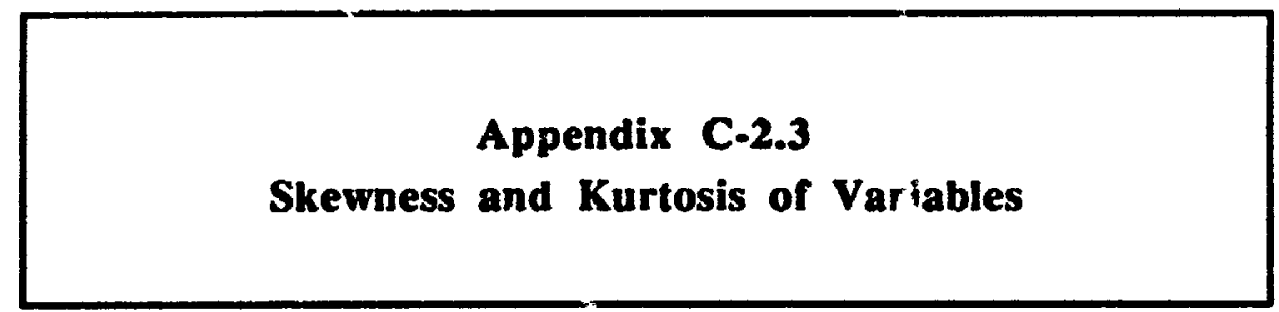




\begin{tabular}{|l|c|c|c|c|c|c|c|c|c|}
\hline VARIABLE & N & M & SD & MIN & MAX & SKEWNESS & Z & KURTOSIS & $Z^{*}$ \\
\hline AGE & 17 & 25.9 & 3.3 & 20 & 32 & 0.2 & -0.9 & -0.7 & -0.5 \\
\hline EDUC & 17 & 15.0 & 2.2 & 12 & 19 & 0.0 & -1.1 & -0.9 & 0.6 \\
\hline MCAT & 17 & 44.4 & 3.1 & 37 & 49 & -0.6 & 0.1 & 0.1 & -0.4 \\
\hline VR & 17 & 43.7 & 4.1 & 36 & 45 & -0.9 & 0.0 & 0.0 & -0.2 \\
\hline NA & 17 & 33.7 & 4.0 & 35 & 48 & -1.2 & 1.1 & 0.9 & -0.3 \\
\hline VR+NA & 17 & 77.4 & 5.6 & 23 & 39 & -1.0 & 0.7 & 0.5 & -0.9 \\
\hline AR & 17 & 41.1 & 2.8 & 63 & 85 & -0.4 & -0.9 & -0.8 & 1.7 \\
\hline CS\&A & 17 & 66.6 & 7.8 & 53 & 79 & 0.0 & -1.2 & -1.0 & -1.3 \\
\hline MR & 17 & 62.5 & 3.9 & 54 & 68 & -0.6 & -0.5 & -0.4 & 0.2 \\
\hline SR & 17 & 51.6 & 4.4 & 43 & 59 & -0.4 & -0.5 & -0.4 & -0.2 \\
\hline FRI+FR2 & 17 & 20.4 & 4.9 & 13 & 29 & 0.5 & -0.9 & -0.8 & -0.6 \\
\hline FRS-FR1 & 17 & 5.0 & 2.3 & 1 & 9 & -0.2 & -0.5 & -0.4 & 0.4 \\
\hline CF1+CF2 & 17 & 111.4 & 11.3 & 4 & 25 & -0.4 & 1.7 & 1.4 & -0.4 \\
\hline CF4-CF1 & 17 & 20.5 & 8.6 & 3 & 36 & -0.2 & -0.3 & -0.3 & -0.2 \\
\hline CFT1+CFT2 & 17 & 157.9 & 41.7 & 106 & 240 & 1.0 & -0.1 & -0.1 & -0.5 \\
\hline CFT4-CFT1 & 17 & -12.2 & 23.7 & -53 & 19 & -0.4 & -0.9 & -0.8 & -0.9 \\
\hline WKMTOT & 17 & 37.8 & 2.7 & 31 & 40 & -1.0 & 0.1 & 0.1 & -0.1 \\
\hline NOAC & 17 & 89.6 & 12.6 & 62.5 & 100 & -0.7 & -0.9 & -0.8 & 0.2 \\
\hline IDENT & 17 & 28.7 & 26.4 & 0 & 75 & 0.2 & -1.4 & -1.2 & -1.1 \\
\hline ALT & 17 & 77.1 & 14.9 & 50 & 100 & -0.1 & -1.1 & -0.9 & -1.2 \\
\hline SP & 17 & 34.6 & 34.9 & 0 & 100 & 0.4 & -1.1 & -1.0 & -1.8 \\
\hline HDG & 17 & 91.5 & 12.0 & 62.5 & 100 & -1.1 & 0.0 & 0.0 & 0.2 \\
\hline POS & 17 & 53.7 & 18.1 & 25 & 87.5 & 0.2 & -0.8 & -0.7 & 0.5 \\
\hline RATF & 16 & 6.7 & 2.4 & 3.5 & 11.2 & 0.5 & -1.1 & -0.9 & 0.3 \\
\hline ACAD & 17 & 89.4 & 4.9 & 80.8 & 96.6 & -0.4 & -1.1 & -0.9 & -1.1 \\
\hline SIM & 17 & 78.1 & 6.5 & 65.5 & 87.3 & -0.5 & -0.7 & -0.6 & 0.1 \\
\hline AVG.RATE & 17 & 113.4 & 21.1 & 69 & 146.7 & -0.4 & -0.2 & -0.2 & -0.5 \\
\hline AVG.OALL & 17 & 4.0 & 1.5 & 1.3 & 6 & -0.4 & -0.7 & -0.6 & -0.5 \\
\hline AVG.PICT & 17 & 3.5 & 1.2 & 1.3 & 5 & -0.5 & -0.9 & -0.8 & 0.6 \\
\hline & & & & & & & & & \\
\hline
\end{tabular}

* If $Z$ exceeds the critical value of $+/-2.86$ the variable is considered to be extremely skewed or kunosed (Tabachnick and Fidell, 1989) 


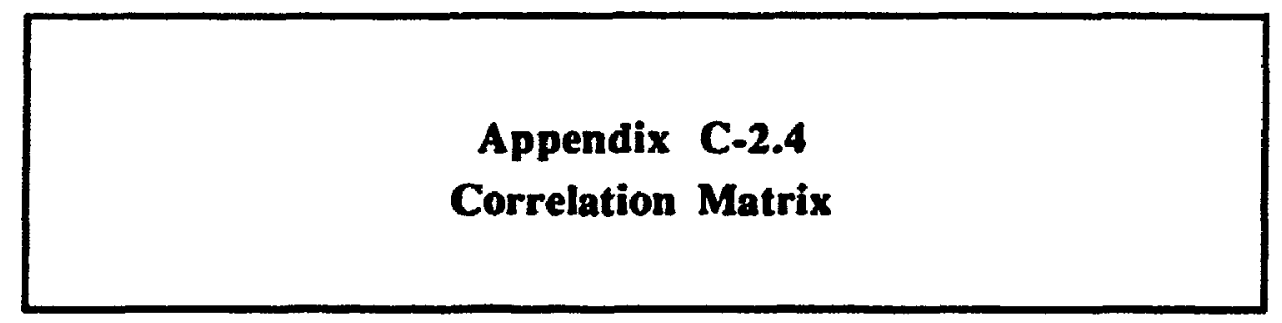




\begin{tabular}{|c|c|c|c|c|c|c|c|c|c|c|c|}
\hline & AGE & EDUC & MCAT & VR & $\mathbf{N}$ & VR+NA & $\mathbf{A R}$ & CSEA & $\mathbf{M R}$ & SR & $F R 1+F R 2$ \\
\hline AGE & 1.00 & & & & & & & & & & \\
\hline EDUC. & 0.22 & 1.00 & & & & & & & & & \\
\hline MCAT & -0.51 & -0.30 & 1.00 & & & & & & & & \\
\hline$\overline{V R}$ & 0.12 & 0.17 & 0.01 & 1.00 & & & & & & & \\
\hline NA & -0.23 & 0.11 & 0.17 & -0.07 & 1.00 & & & & & & \\
\hline$\sqrt{R+N A}$ & -0.08 & 0.21 & 0.13 & 0.69 & 0.67 & 1.00 & & & & & \\
\hline$\overline{A R}$ & 0.34 & 0.23 & -0.24 & 0.32 & 0.39 & 0.52 & 1.00 & & & & \\
\hline CSLA & 0.03 & -0.13 & 0.15 & 0.04 & 0.32 & 0.26 & .0 .08 & 1.00 & & & \\
\hline MR & -0.08 & 0.16 & 0.16 & 0.20 & 0.00 & 0.15 & -0.28 & .0 .04 & 1.00 & & \\
\hline $\begin{array}{l}\mathbf{S R} \\
\end{array}$ & -0.27 & -0.15 & 0.57 & 0.35 & -0.35 & 0.01 & .0 .10 & .0 .18 & 0.09 & 1.00 & \\
\hline FR1+FR2 & -0.41 & 0.16 & -0.04 & 0.30 & 0.02 & 0.23 & 0.24 & -0.19 & .0 .03 & 0.23 & 1.00 \\
\hline FRS-FR1 & 0.67 & -0.14 & 0.04 & -0.22 & -0.20 & -0.31 & 0.05 & 0.09 & 0.12 & -0.21 & -0.65 \\
\hline $\mathrm{CF1}+\mathrm{CF} 2$ & 0.27 & -0.44 & 0.03 & 0.48 & -0.39 & 0.08 & .0 .03 & 0.16 & -0.07 & 0.49 & -0.11 \\
\hline CF4-CF1 & 0.21 & -0.59 & 0.00 & 0.06 & -0.58 & .0 .38 & -0.25 & 0.04 & -0.17 & 0.41 & -0.29 \\
\hline CFT1+CFT2 & -0.17 & -0.05 & 0.34 & 0.32 & 0.02 & 0.25 & 0.23 & .0 .01 & .0 .40 & 0.44 & 0.06 \\
\hline CFT4-CFT1 & 0.08 & 0.15 & -0.07 & 0.15 & 0.05 & 0.15 & 0.12 & .0 .13 & 0.34 & -0.17 & 0.00 \\
\hline WKMTIOT & 0.00 & 0.13 & 0.08 & 0.12 & 0.54 & 0.48 & 0.23 & .0 .06 & 0.19 & -0.08 & 0.19 \\
\hline NOAC & -0.17 & -0.30 & 0.42 & 0.64 & -0.27 & 0.28 & -0.20 & .0 .13 & 0.24 & 0.58 & 0.16 \\
\hline IDENT & -0.33 & -0.13 & 0.19 & -0.14 & 0.03 & -0.09 & 0.04 & 0.15 & -0.25 & 0.08 & 0.49 \\
\hline ALT & -0.08 & 0.33 & 0.06 & 0.14 & 0.05 & 0.14 & 0.05 & 0.19 & -0.07 & -0.06 & -0.02 \\
\hline SP & -0.28 & -0.05 & 0.23 & 0.18 & 0.20 & 0.28 & 0.19 & 0.37 & 0.01 & 0.04 & 0.45 \\
\hline HDG & -0.43 & -0.53 & 0.44 & 0.10 & -0.20 & -0.07 & .0 .22 & .0 .08 & 0.07 & 0.30 & 0.03 \\
\hline POS & -0.21 & -0.28 & 0.59 & 0.30 & 0.31 & 0.44 & 0.18 & 0.23 & 0.15 & 0.24 & -0.05 \\
\hline RATE & -0.24 & -0.33 & -0.08 & -0.35 & 0.36 & 0.01 & .0 .45 & 0.55 & -0.11 & -0.44 & -0.32 \\
\hline$\overline{\mathrm{ACAD}}$ & 0.00 & -0.30 & 0.18 & 0.13 & 0.02 & 0.11 & -0.37 & 0.35 & 0.08 & -0.04 & -0.37 \\
\hline $\sin$ & -0.09 & -0.31 & -0.08 & 0.12 & -0.45 & -0.24 & .0 .51 & 0.21 & 0.20 & -0.10 & -0.02 \\
\hline AVG.RATE & 0.16 & -0.28 & -0.22 & 0.07 & -0.44 & -0.27 & -0.42 & 0.15 & 0.05 & -0.30 & -0.27 \\
\hline AVG.OALL & 0.03 & $-0.2 i$ & -0.23 & 0.04 & -0.40 & -0.26 & -0.46 & 0.19 & 0.05 & -0.33 & -0.19 \\
\hline AVG.PICT & -0.03 & -0.25 & -0.18 & 0.04 & -0.43 & -0.28 & -0.48 & 0.21 & 0.16 & -0.25 & -0.07 \\
\hline
\end{tabular}




\begin{tabular}{|c|c|c|c|c|c|c|c|c|}
\hline & FRS-FRI & CFItCF2 & CF4-CFI & CFT1+CFT2 & CFT4-CFT1 & WhMrTor & NOAC & DENT \\
\hline FRS-FRI & 1.00 & & & & & & & \\
\hline $\mathrm{CF} 1+\mathrm{CF} 2$ & 0.10 & 1.00 & & & & & & \\
\hline CF4-CFI & 0.27 & 0.81 & 1.00 & & & & & \\
\hline CFT1+CFT2 & -0.29 & 0.17 & .0 .06 & 1.00 & & & & \\
\hline CFTA-CFTI & 0.20 & -0.10 & 0.02 & -0.53 & 1.00 & & & \\
\hline WKMTOT & .0 .15 & -0.16 & -0.50 & 0.25 & .0 .29 & 1.00 & & \\
\hline NOAC & -0.12 & 0.64 & 0.42 & 0.19 & 0.07 & 0.06 & 1.00 & \\
\hline IDENT & .0 .27 & .0 .12 & -0.07 & -0.02 & 0.19 & -0.05 & 0.00 & 1.00 \\
\hline ALT & 0.03 & .0 .15 & -0.12 & .0 .15 & 0.04 & .0 .28 & 0.05 & 0.08 \\
\hline $\mathbf{S P}$ & -0.20 & 0.05 & -0.04 & 0.01 & 0.29 & 0.03 & 0.25 & 0.68 \\
\hline FDG & -0.09 & 0.18 & 0.29 & 0.24 & -0.04 & -0.10 & 0.58 & 0.30 \\
\hline POS & 0.10 & 0.05 & -0.05 & 0.44 & -0.07 & 0.39 & 0.40 & 0.07 \\
\hline RATE & -0.16 & 0.00 & 0.08 & .0 .16 & .0 .27 & 0.10 & -0.13 & -0.04 \\
\hline$\overline{A C A D}$ & 0.10 & 0.39 & 0.43 & -0.32 & 0.44 & -0.33 & 0.43 & 0.15 \\
\hline $\sin$ & -0.02 & 0.27 & 0.43 & .0 .08 & 0.24 & -0.41 & 0.41 & 0.18 \\
\hline AVG.RATE & 0.19 & 0.28 & 0.42 & -0.32 & 0.39 & -0.55 & 0.25 & 0.06 \\
\hline AVG.OAUL & 0.06 & 0.19 & 0.33 & -0.31 & 0.36 & -0.54 & 0.18 & 0.15 \\
\hline AVG.PICT & 0.02 & 0.18 & 0.32 & -0.34 & 0.40 & -0.52 & 0.20 & 0.27 \\
\hline
\end{tabular}

\begin{tabular}{|l|c|c|c|c|c|c|c|c|c|c|}
\cline { 2 - 12 } \multicolumn{1}{c|}{} & ALT & SP & HDG & POS & RATE & ACAD & SIM & AV.RATE & AV.OALL & AV.PICT \\
\hline ALT & 1.00 & & & & & & & & & \\
\hline SP & 0.15 & 1.00 & & & & & & & & \\
\hline HDG & 0.11 & 0.45 & 1.00 & & & & & & & \\
\hline POS & 0.09 & 0.42 & 0.61 & 1.00 & & & & & & \\
\hline RATE & 0.06 & 0.07 & 0.10 & 0.05 & 1.00 & & & & & \\
\hline ACAD & 0.13 & 0.23 & 0.28 & 0.04 & 0.42 & 1.00 & & & & \\
\hline SIM & -0.34 & 0.25 & 0.54 & 0.01 & 0.17 & 0.45 & 1.00 & & & \\
\hline AVG.RATE & -0.06 & 0.15 & 0.30 & -0.20 & 0.23 & 0.67 & 0.82 & 1.00 & & \\
\hline AVG.OAL & -0.04 & 0.18 & 0.31 & -0.21 & 0.30 & 0.63 & 0.84 & 0.98 & 1.00 & \\
\hline AVG.PICT & -0.08 & 0.31 & 0.33 & -0.19 & 0.24 & 0.59 & 0.89 & 0.94 & 0.97 & 1.00 \\
\hline
\end{tabular}




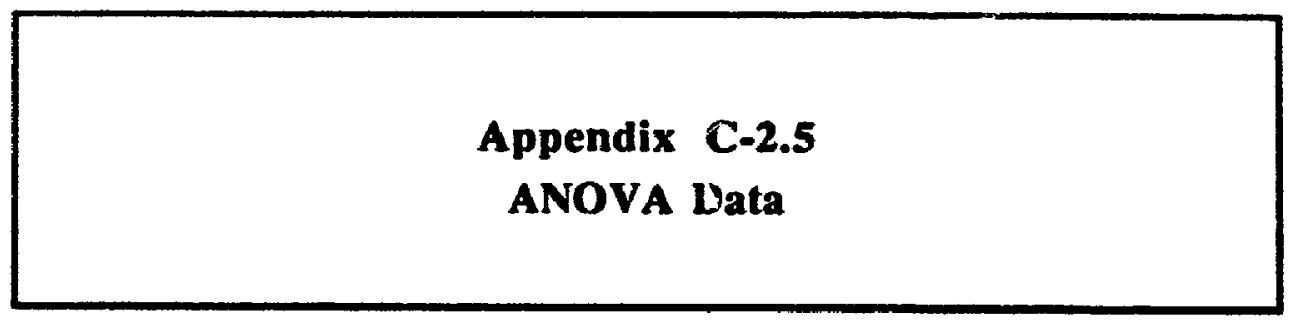


C-1.5.A: ANOVA Data for Accuracy of Recall

\begin{tabular}{|l|c|c|c|c|c|}
\hline \multicolumn{1}{|c|}{ TEST } & SS & MS & df & F & $\begin{array}{c}\text { SIGN- } \\
\text { FICANCE }\end{array}$ \\
\hline BETWEEN GROUPS & 49565.57 & 12391.39 & 4 & 23.63 & P<.0001 \\
\hline WITHIN GROUPS & 41954.12 & 524.43 & 80 & & \\
\hline TOTAL & 91519.69 & & 84 & & \\
\hline
\end{tabular}

\section{C-2.5.B: Scheffé Test Data}

\begin{tabular}{|l|c|c|c|c|}
\hline \multicolumn{1}{|c|}{ TEST } & MEAN A & MEAN B & SCHEFFE F & $\begin{array}{c}\text { SIGNI- } \\
\text { FICANCE }\end{array}$ \\
\hline IDENT VS. SPEED & 28.65 & 34.65 & 0.15 & NS \\
\hline IDENT VS HEADING & 28.65 & 91.53 & 16.02 & $\mathrm{p}<.05$ \\
\hline IDENT VS. POSITION & 28.65 & 53.76 & 2.56 & $\mathrm{p}<.05$ \\
\hline IDENT VS. ALTITUDE & 28.65 & 77.23 & 9.57 & $\mathrm{p}<.05$ \\
\hline SPEED VS. HEADING & 34.65 & 91.53 & 13.11 & $\mathrm{p}<.05$ \\
\hline SPEED VS. POSITION & 34.65 & 53.76 & 1.48 & $\mathrm{NS}$ \\
\hline SPEED VS ALTITUDE & 34.65 & 77.23 & 7.35 & $\mathrm{p}<.05$ \\
\hline HEADING VS. POSITION & 91.53 & 53.76 & 5.78 & $\mathrm{p}<.05$ \\
\hline HEADING VS. ALTITUDE & 91.53 & 77.23 & 0.83 & $\mathrm{NS}$ \\
\hline POSITION VS. ALTTTUDE & 53.76 & 77.23 & 2.23 & $\mathrm{NS}$ \\
\hline
\end{tabular}




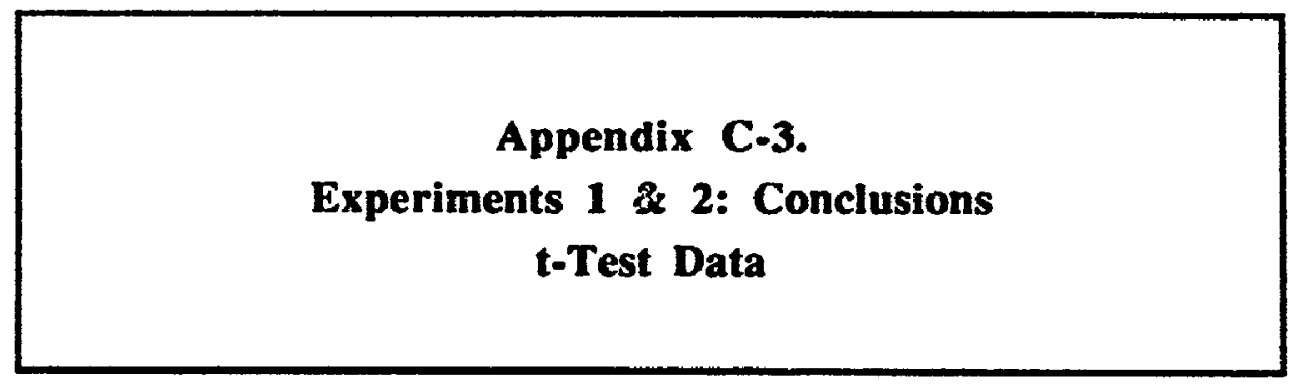




\begin{tabular}{|l|c|c|c|c|c|}
\hline \multicolumn{1}{|c|}{ TEST } & $\begin{array}{c}\text { MEANS: } \\
\text { RADAR }\end{array}$ & $\begin{array}{c}\text { MEANS: } \\
\text { PROCEDURAL }\end{array}$ & df & t & $\begin{array}{c}\text { SIGNI- } \\
\text { FICANCE }\end{array}$ \\
\hline POSITION & 85.9 & 53.7 & 51 & -5.26 & p $<.05$ \\
\hline ALTTTUDE & 73.4 & 77.1 & 46 & -0.71 & NS \\
\hline HEADING & 83.0 & 91.5 & 48 & 2.09 & p $<.05$ \\
\hline SPEFD & 52.9 & 34.5 & 51 & -1.67 & NS \\
\hline IDENTIFIER & 55.2 & 28.7 & 51 & -3.44 & p $<.05$ \\
\hline
\end{tabular}




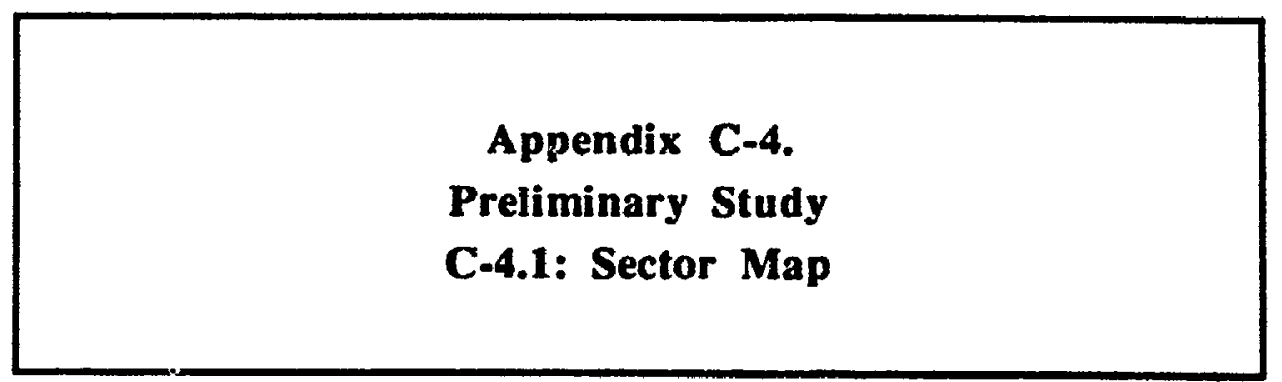




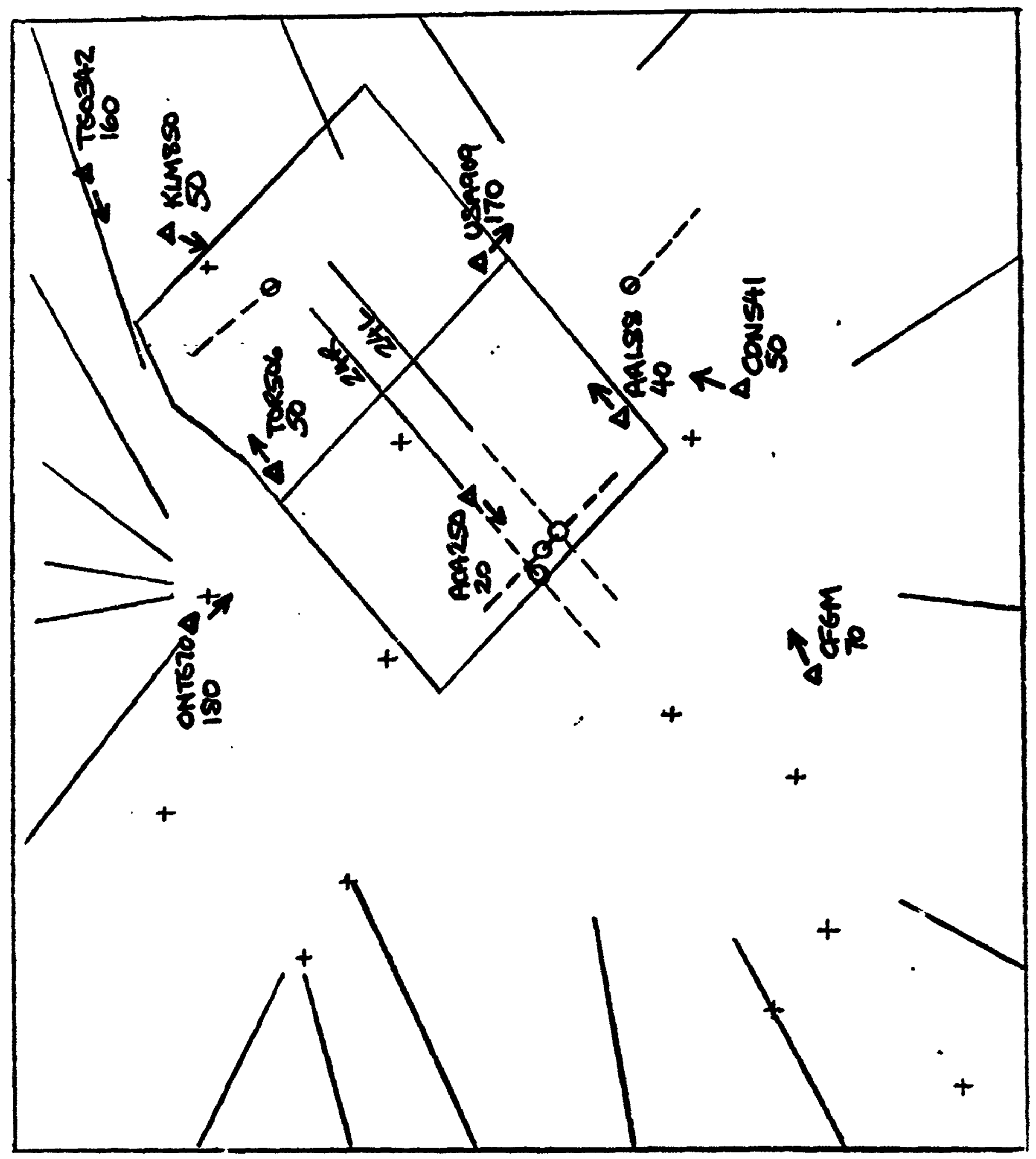




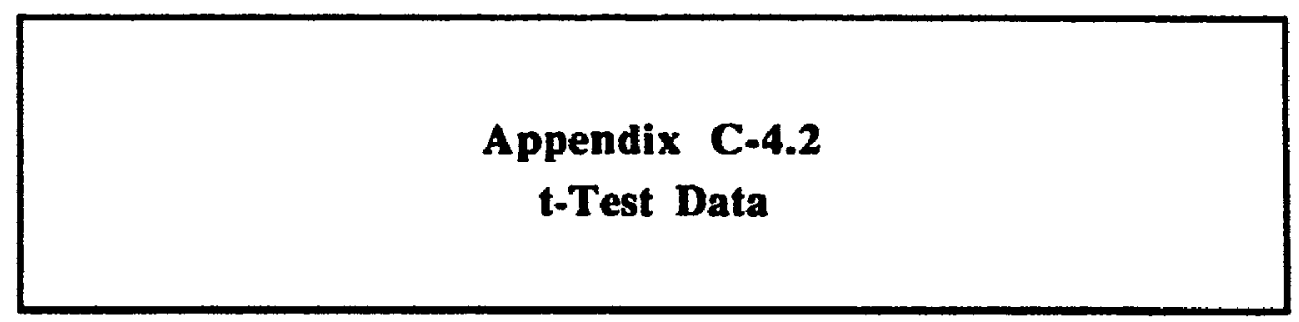




\begin{tabular}{|c|c|c|c|c|c|}
\hline TEST & $\begin{array}{c}\text { MEANS: } \\
\text { CONTROLLERS }\end{array}$ & $\begin{array}{c}\text { MEANS: } \\
\text { NON- } \\
\text { CONTROLLERS }\end{array}$ & df & $\mathbf{t}$ & $\begin{array}{l}\text { SIGNI- } \\
\text { FICANCE }\end{array}$ \\
\hline \multicolumn{6}{|c|}{ RECALL AND CATEGORIZATION TASKS } \\
\hline RECALL RATE & 23.9 & 16.1 & 5 & 1.11 & NS \\
\hline RECALL RATE (W/O C2\&C4) & 33.9 & 16.1 & 7 & 4.48 & $p<.05$ \\
\hline ABSOLUTE 2D & 3.16 & 4.32 & 6 & -1.94 & NS \\
\hline ABSOLUTE 3D & 3.26 & 5.1 & 7 & -3.09 & $0<.05$ \\
\hline BALLPARK 2D & 67.78 & 57.16 & 9 & 0.86 & NS \\
\hline BALLPARK 3D & 67.78 & 38.09 & 10 & 2.79 & $p<.05$ \\
\hline BALLPARK 2D (W/OC4) & 76.4 & 57.16 & 6 & 2.15 & NS \\
\hline BALLPARK 3D (W/OC4) & 73.63 & 38.09 & 6 & 3.94 & $p<.05$ \\
\hline SIMILARITY JUDGEMENTS & 50.01 & 80.0 & 9 & -3.09 & $p<.05$ \\
\hline \multicolumn{6}{|l|}{ SPATIAL TASKS } \\
\hline FREE ALT. JUDGEMENTS & 357.29 & 349 & 9 & 0.17 & $\mathbf{N S}$ \\
\hline SLIDER PLACEMENT & 5.75 & 5.75 & 8 & 0.00 & NS \\
\hline HEAD TURNS & 1.52 & 2.11 & 10 & -2.29 & $p<.05$ \\
\hline RESPONSE TIME & 15.43 & 18.76 & 10 & -1.26 & NS \\
\hline \multicolumn{6}{|l|}{ MODEL BUILDING } \\
\hline $2 \mathrm{D}$ & 1.35 & 1.48 & 9 & -0.62 & NS \\
\hline 3D & 2.70 & 2.29 & 7 & -0.94 & NS \\
\hline \multicolumn{6}{|l|}{ TWO MINUTE } \\
\hline $2 \mathrm{D}$ & 2.9 & 2.56 & 10 & 0.86 & NS \\
\hline $3 D$ & 3.98 & 3.67 & 9 & 0.45 & NS \\
\hline \multicolumn{6}{|l|}{ TWO MINUTE DIFFERENCE } \\
\hline $2 \mathrm{D}$ & 1.56 & 1.09 & 10 & 1.79 & NS \\
\hline $3 \mathrm{D}$ & 1.26 & 1.39 & 10 & -0.28 & NS \\
\hline \multicolumn{6}{|l|}{ FIVE MINUTE } \\
\hline $2 \mathrm{D}$ & 3.56 & $\mathbf{3 . 8 1}$ & 10 & -0.25 & $\overline{N S}$ \\
\hline $3 \mathrm{D}$ & 4.24 & 4.53 & 9 & -0.25 & NS \\
\hline \multicolumn{6}{|l|}{ FIVE MINUTE DIFFERENCE } \\
\hline $2 \mathrm{D}$ & 2.24 & 2.31 & 7 & -0.08 & NS \\
\hline $3 \mathrm{D}-1$ & 1.56 & 2.23 & 10 & -0.65 & NS \\
\hline \multicolumn{6}{|l|}{ ARRIVALS VS. INTERSECTIONS } \\
\hline \multicolumn{6}{|l|}{ PRESENT } \\
\hline $2 \mathrm{D}$ & -0.20 & 0.03 & 10 & -0.53 & NS \\
\hline $3 \mathrm{D}$ & -0.94 & 0.24 & 10 & -3.43 & $p<.05$ \\
\hline \multicolumn{6}{|l|}{ TWOMINUTE } \\
\hline $2 \mathrm{D}$ & -0.21 & -0.53 & $\mathbf{5}$ & 0.49 & NS \\
\hline 3D & 0.08 & -0.70 & 10 & 1.50 & NS \\
\hline 3D(WITHOUTC2) & 0.355 & 0.534 & 9 & 2.32 & $0<.05$ \\
\hline \multicolumn{6}{|c|}{ CONFLICTS VS. NON-CONFLICTS } \\
\hline \multicolumn{6}{|c|}{ PRESENT } \\
\hline $2 \mathrm{D}$ & -0.69 & -0.01 & 10 & -1.24 & NS \\
\hline 3D & -0.86 & -0.09 & 10 & -1.79 & NS \\
\hline \multicolumn{6}{|l|}{ TWOMINUTE } \\
\hline $2 \mathrm{D}$ & 0.19 & 0.36 & 10 & -0.40 & NS \\
\hline 3D & -0.58 & 0.07 & 10 & 1.66 & NS \\
\hline
\end{tabular}




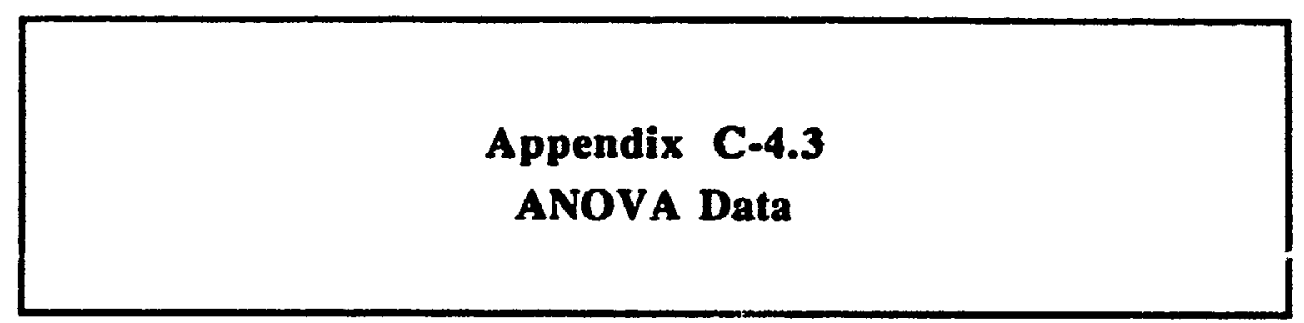


C-4.3.A: Recall Consistency

\begin{tabular}{|l|c|c|c|c|c|}
\hline \multicolumn{1}{|c|}{ VARIABLE } & $\begin{array}{c}\text { VARIANCE: } \\
\text { CONTROLLERS }\end{array}$ & $\begin{array}{c}\text { VARIANCE: } \\
\text { NON- } \\
\text { CONTROLLERS }\end{array}$ & di & F & $\begin{array}{c}\text { SIGNI- } \\
\text { FICANCE }\end{array}$ \\
\hline RATE (W/OC2 \& C4) & 68.4 & 17.5 & 6,2 & 3.9 & NS \\
\hline ABSOLUTE 2D & 0.15 & 2.59 & 6,4 & 17.46 & p<.05 \\
\hline ABSOLUTE 3D & 0.18 & 2.23 & 6,4 & 12.51 & p<.05 \\
\hline BALLPARK 2D & 373.48 & 553.47 & 6,4 & 1.48 & NS \\
\hline BALLPARK 3D & 18.11 & 529.94 & 6,4 & 2.82 & NS \\
\hline BALLPARK 2D (W/OC3) & 2.61 & 553.47 & 6,3 & 211.77 & p<.01 \\
\hline BALLPARK 3D (W/OC3) & 23.05 & 529.94 & 6,3 & 22.98 & p<.05 \\
\hline
\end{tabular}

\section{C-4.3.B: Recall Accuracy: Attributes X Groups}

\begin{tabular}{|l|c|c|c|c|c|}
\hline \multicolumn{1}{|c|}{ TEST } & SS & MS & df & F & $\begin{array}{c}\text { SIGNI- } \\
\text { FICANCE }\end{array}$ \\
\hline GROUP(A) & 520.84 & 260.42 & 2 & 0.33 & NS \\
\hline ATTRIBUTE(B) & 10127.88 & 2531.97 & 4 & 3.24 & P<.05 \\
\hline AB & 5840.26 & 730.03 & 8 & 0.93 & NS \\
\hline ERROR & 35164.57 & 782.43 & 45 & & \\
\hline
\end{tabular}




\section{References}

Anshel, M. H., \& Ortiz, M. (1986). Effect of coding strategies on movement extent as a function of cognitive style. Perceptual and Motor Skills, 63, $1311-1317$.

Baird, J. C. (1979). Studies of the cognitive representation of spatial relations: $I$. Overview. Joumal of Experimental Psychology: General, 108, 90-91.

Baird, J. C. (1979). Studies of the cognitive representation of spatial relations: II. A familiar environment. \ournal of Experimental Psychology: General, 108, 92-106.

Bennett, G. K., Seashore, H. G., \& Wesman, A. G. (1988). Differential Aptitude Tests. Toronto, ON: Harcourt, Brace, Jovanovich.

Bisseret, A. (1970). Mémoire operationelle et structure du travail. Bulletin de Psychologie, 24, 280-294.

Bisseret, A. (1971). Analysis of mental processes involved is air traffic control. Ergonomics, 14, 565-570.

Bisseret, A. (1981). Application of signal detection theory to decision making in supervisory control. Ergonomics, 24, 81-94.

Boone, J. O. (1984). 1976-1978 selection study of new appointees to the ATC occupation. In S. B. Sells, J. T. Dailey, \& E. W. Pickerel (Eds.), Selection of air traffic controllers (Report No. FAA-AM-84-2) (pp. 375-395). Washington, DC: FAA.

Brokaw, L. D. (1984). Early research on controller selection: 1941-1963. In S. B. Sells, J. T. Dailey, \& E. W. Pickerel (Eds.), Selection of air traffic controllers (Report No. FAA-AM-84-2) (pp. 39-78). Washington, DC: FAA.

Caplan, B. (1984). Sex differences in cognitive strategic preference among medical students. Perceptual and Motor Skills, 58, 279-285.

Chase, W. G., \& Ericsson, K. A. (1982). Skilled memory. In J. R. Anderson (Ed.), Cognitive Skills and their Aquisition. Hillsdale, NJ: Lawrence Erlbaum.

Chase, W. G., \& Simon, H. A. (1973). Perception in chess. Cognitive Psychology, 4 , 55-81.

Cobb, B. B., Nelson, P. L., \& Mathews, J. J. (1973). The relationships of age and ATC experience to job performance ratings of terminal area traffic controllers (Report No. FAA-AM-73-7). Washington, DC: Federal Aviation Administration.

Coeterier, J. F. (1971). Individual strategies in ATC freedom and choice. Ergonomics, $14,579-564$.

Cooper, L. A., \& Mumaw, R. J. (1985). Spatial aptitude. In R. F. Dillon \& R. R. Schmeck (Eds.), Individual Differences in Cognition (pp. 67-94). New York: Academic Press. 
Dailey, J. T. (1984). Characteristics of the air traffic contuller. In S. B. Sells, J. T. Dailey, \& E. W. Pickerel (Eds.), Selection of air traffic controllers (Report No. FAAAM-84-2) (pp. 128-141). Washington, DC: FAA.

de Groot, A.D. (1965). Thought and Choice in Chess. The Hague: Mouton.

Edenborough, R. A. (1972, November). Human factors evaluation of labelled radar displays. Aerospace Medicine, pp. 1190-1193.

Egan, D. E., \& Gomez, L. M. (1985). Assaying, isolating, and accommodating individual differences in learning a complex skill. In R. F. Dillon (Ed.), Individual Differences in Cognition (Vol. 2) (pp. 173-217). New York: Academic Press.

Ericsson, K. A., \& Simon, H. A. (1980). Verbal reports as data. Psychological Review, 87, 215-251.

Falzon, P. (1982). Display strictures: Compatibility with the operator's mental representation and reasor.ing processes. In G. Johannsen (Ed.). Broceedings of the Second European Ann' al Conference of Human Decision Making and Manual Control (pp. 287-305). Wac'،berg-Werhoven, W. Germany: FGAN/FAT.

Farber, J., \& Rosinski, R. R. (1978). Geometric transformation of pictured space. Perception, 1, 269-282.

Finke, R. A., \& Shepard, R. N. (1986). Visual functions of mental imagery. In K. R. Boff, L Kaufman, \& J. P. Thomas (Eds.) Handbook of Perceprion and Human Performance (pp. 37-1 to 37-55). New York, NY: John Wiley.

Fitts, P. M. (1951). Human engineering for an effective air-navigation and traffic-control System (Report No. 593420). Washington, DC: National Research Council.

Fogel, L. J. (1963). Biotechnology: Concepts and Applications. Englewood Cliffs, NJ: Prentice-Hall.

Fraker, M. L. (1989). Attention allocation in situation awareness. Broceedings of the Human Factors Society (pp. 1396-1400). Santa Monica, CA: Human Factors Society.

Galin, D., \& Omstein, R. (1974). Individual differences in cognitive styie: I. Reflective eye movements. Neuropsychologia, 12, 367-376.

Gopher, D. (1982). A selective attention test as a predictor of success in flight training. Human Factors, 24, 173-183.

Greener, J. M. (1984). Post-training criterion measures in validation of controller selection procedures. In S. B. Sells, J. T. Dailey, \& E. W. Pickrel (Eds.), Selection of air traffic controllers (Report No. FAA-AM-84-2) (pp. 241-262). Washington, DC: FAA.

Haber, R. N. (1981). The power of visual perceiving. Journal of Mental Imagery, 5, 140. 
Hammond, N. (1987). Frinciples from the psychology of skill acquisition. In M. M. Gardiner \& B.Christie (Eds.), Applying Connitive Psvchology to User-Interface Design (pp. 163-188). Chichester, UK: John Wiley \& Sons.

Hanisch, K. A., Kramer, A. F., Hulin, C. L., \& Schumacher, R. (1988). Novice-expert differences in the cognitive representation of system features: Mental models and verbalization knowledge. Proceedings of the Human Factors Socieiy (pp. 219-223). Santa Monica, CA: Human Factors Society.

Hart, S. G., \& Loomis, L. L. (1980). Evaluation of the potential format and content of a cockpit display of traffic information. Human Factors, 22, 591-604.

Hitch, G. J. (1987). Principles from the psychology of memory (Part 1: Working memory). In M. M. Gardiner \& B.Christie (Eds.), Applving Cognitive Psychology 10 User-Interface Design (pp. 120-134). Chichester, UK: John Wiley \& Sons.

Hopkin, V. D. (1972). Flow diagrams. In R. K. Bernotat, \& K. P. Gartner (Eds.) Displays and Controls (pp. 191-212). Amsterdam: Swets and Zeitlinger, N.V.

Hopkin, V. D. (1982). Human factors in air traffic control (AGARDograph No. 275). Neuilly-Sur-Seine, France: AGARD.

Howell, D. C. (1987). Statistical Methods for Psychology. Boston, MA: Duxbury Press.

Jagacinski, R. J. (1978). Describing the human operator's internal model of a dynamic system. Human Factors, 20, 425-433.

Johnson-Laird, P. N. (1983). Mental Models. Cambridge, England: Cambridge University Press.

Kelley, C. R. (1972). Display layout. In R. K. Bernotat \& K. P. Gartner (Eds.), Displays and Controls (pp. 41-52). Amsterdam: Swets and Zeitlinger NV.

Johannsen, G., \& Rouse, W. (1979). Mathematical concepts for modeling human behavior in complex man-machine systems. Human Factors. 21, 733-747.

Kheraj, R. J. (1987). The selectivity of cognitive processing in an air-traffic control radar task. Unpublished doctoral dissertation, Queen's University, Kingston.

Kirchner, J. H., \& Laurig, W. (1971). The human operator in air traffic control systems. Ergonomics, 14, 549-556.

Kieras, D. E., \& Bovair, S. (1984). The role of a mental model in learning to operate a device. Cognitive Science, 8, 155-173.

Lafon-Milon, M. T. (1981). Représentation mentale de la separation verticale au cours du diagnostic dans le controle aerien: III Représentation des états futurs (Report No. CO 8107 R66). Rocquencourt, France: INRIA. 
Landis, D., Silver, C. A., Jones, J. M., \& Messick, S. (1967). Level of proficiency and multidimensional viewpoints about problem similarity. Joumal of Applied Bsychology, 51, 216-222.

Lapan, Y. A. (1985). Spatial representation and the activity of the air traffic controller. Vesmik Moskovskogo Universiteta Seriva 14 Psikhologiva, 14, 68-70.

Leplat. J., \& Bisseret, A. (1965). Analysis of the processes involved in the treatment of information by the air traffic controller. The controller, 5, 13-22.

Loftus, G. R. (1979). Short-term memory factors in ground controller/pilot communication. Human Factors, 21, 169-181.

Lynch, K. (1960). The Image of the City. Cambridge, MA: Technology Press.

Manktelow, K., \& Jones, J. (1987). Principles from the psychology of thinking and mental models. In M. M. Gardiner \& B.Christie (Eds.), Applying Cogritive Psychology to User-Interface Desien (pp. 83-117). Chichester, UK: John Wiley \& Sons.

McGee, M. G. (1979). Human spatial abilities: Psychometric studies and environmental, genetic, hormonal, and neurological influences. Psychological Bulletin, 86, 889-918.

McGreevy, M. W., \& Ellis, S. R. (1986). The effect of perspective geometry on judged direction in spatial information instruments. Human Faciors, 28, 439-456.

Means, B., Mumaw, R., Roth, C., Schalger, M., McWilliams, E., Gagne, E., Rice, V., Rosenthal, D., \& Heon, S. (1988). ATC training analysis study: Desjen of the nextgeneration A TC training system (OPM Work Order No. 342-036). Richmond, VA: HumRRO International.

Moray, N. (1980). Human information processing and supervisory control (Report No. AD-A092 840). Cambridge, MA: MTT.

Moray, N., \& Reid, L. D. (1972). A review of models of the air rraffic control system (Report No. 5). Toronto, ON: University of Toronto-York University.]

Murphy, E. D., Reaux, R. A., Stewart, L. J., Coleman, W. D., \& Harwood, K. (1989). Modeling air traffic controller performance in highly automated environments. Proceedings of the Human Factors Society (pp. 47-51). Santa Monica, CA: Human Factors Society.

Nisbett, R. E., \& Wilson, T. D. (1977). Telling more than we can know: Verbal reports on mental processes. Psychological Review, 84, 231-259.

Na:borough-Hall, C. S. (1985). Computer assistance: Implications for memory performance as a function of control responsibility assigned to man. In Analysis, Desien and Evaluation of Man-Machine Systems. Second IFAC/IFIP/IFORS/IER Conference, IFAC. 
Narborough-Hall, C. S. (1987). Automation: Implication for knowledge retention as a function of operator control responsibility. In D. Draper \& R. Winder (Eds.), People and Computers: Proceedings of the Thind Conference of the British Compurer Society. Human Computer Interaction Specialist Group (pp. 1-14). Cambridge: Cambridge University Press.

Norman, D. A. (1983). Some observations on mental models. In D. Gentner \& A. L. Stevens (Eds.). Mental Models (pp. 241-244). Hillsdale, N.J.: Erlbaum.

Norman, D. A. (1986). Cognitive engineering. In D. A. Norman and S. W. Draper (Eds.), User Centered System Desion (pp. 31-61). Hillsdale, NJ: Lawrence Erlbaum.

O'Conner, J. (1972). Developmental changes in abstractness and moral reasoning. Dissertation Abstracts International, 32, 4107a.

O'Hare, D. (1976). Individual differences in perceived similarity and preference for visual art: A multidimensional scaling analysis. Perception and Psychophysics, 20, 445-452.

Palmer, E. A., Jago, S. J., Baty, D. L., \& O'Conner,S. L. (1980). Perception of horizontal aircraft separation on a cockpit display of traffic information. Human Factors, 22, 605-620.

Pinker, S. (1980). Mental imagery and the third dimension. Journal of Exrerimental Psychology: General, 109, 354-371.

Pinker, S. (1984). Visual Cognition: An introduction. Cognition, 18, 1-63.

Pinker, S., \& Finke, R. A. (1980). Emergent two-dimensional patterns in images rotated in depth. Joumal of Experimental Psuchology: Human Perception and Performance, 6 , 244-264.

Pinker, S., \& Kosslyn, S. M. (1978). The representation and manipulation of threedimensional space in mental images. Joumal of Mental Imagery, 2, 69-84.

Ponds, R. W., Brouwer, W. H., \& van Wolffelaar, P. C. (1988). Age differences in divided attention in a simulated driving task. Joumal of Gerontology, 43, 151-156.

Rasmussen, J. (1979). On the structure of knowled ge: A motphology of mental models in a man-machine system context (Report No. RISO-M-2192). Roskilde, Denmark: Riso National Laboratory.

Richardson, A. (1977). Verbalizer-visualizer: A cognitive style dimension. Journal of Mental Imagery, 1, 109-125.

Roberts, F. S., \& Suppes, P. (1967). Some problems in the geometry of visual perception. Synthese, 17, 173-201.

Rouse, W. B., \& Morris, N. M. (1986). On looking into the black box: Prospects and limits in the search for mental models. Psychological Bulletin, 100, 349-363. 
Rumelhart, D. E. (1977). Introduction to Human Information Processing. New York: John Wiley \& Sons.

Schvaneveldt, R. W., Durso, F. T., Goldsmith, T. E., Breen, T. J., \& Cooke, N. M. (1985). Measuring the structure of expertise. International Joumal of Man-Machine Studies, 23, 699-728.

Sells, S. B., \& Pickrel, E. W. (1984). Summary of the research on the experimental battery: Recommendations for adoption and further research. In S. B. Sells, J. T. Dailey, \& E. W. Pickrel (Eds.), Selection of Air Traffic Controllers, FAA-AM-84-2 (pp. 543-547). Washington, DC: FAA.

Sheehan, P. W., Ashton, R., \& White, K. (1983). Assessment of mental imagery. In A. A. Sheikh (Ed.), Imagery: Current Theory, Research, and Application (pp. 184-221). New York, NY: John Wiley and Sons.

Shepard, R. N. (1962). Analysis of proximities as a technique for the study of information processing in man. Human Factors, 5, 33-48.

Shepard, R. N., \& Metzler, J. (1971). Mental rotation of three-dimensional objects. Science, 171, 701-703.

Singleton, W. T. (1978). The Analysis of Practical Skills. Lancaster, UK: MTP.

Smith, E. R., \& Miller, F. D. (1978). Limits on perception of cognitive processes: A reply to Nisbett and Wilson. Psychological Review, 85, 355-362.

Soede, M., \& Coeterier, J. F. (1971). Time analyses of the tasks of approach controllers in ATC. Ergonomics, 14, 591-601.

Solokov, A. N. (1977). Inner Speech and Thoughts. New York: Plenum Press.

Sperandio, J. (1971). Variation of operator's strategies and regulating effects on workload. Ergonomics, 14, 571-577.

Sperandio, J. (1978). The regulation of working methods as a function of work-load among air traffic controllers. Ergonomics, 21, 195-202.

Stevens, S. S. (1966). Handbook of Experimental Psychology. New York: John Wiley \& Sons.

Stigler, J. W. (1984). "Mental abacus": The effect of abacus training on Chinese children's mental calculation Cognitive Psychology, 16, 145-176.

Tabachnick, B. G., \& Fidell, L. S. (1983). Using Multivariate Statistics. New York, NY: Harper and Row.

Thijs, W. L. Th. (1982). On and beyond consciousness: Notes on cognition in a fault management context. In G. Joliannsen (Ed.), Proceedings of the Second European Annual Conference of Human Decision Making and Manual Control (pp. 267-283). Wachtberg-Werhoven, W. Germany: FGAN/FAT. 
Thorndyke, P. W. (1980). Performance Models for Spatial and Locational Cognition (Technical Report R-2676-ONR). Washington, DC: Rand Corporation.

Tombaugh, T., \& Schmidt, W. (1989). General learning and memory battery. Personal Communication.

Veldhuyzen, W., \& Stassen, H. G. (1977). The internal model concept: An application to modeling human control of large ships. Human Factors, 19, 367-380.

Velleman, P. F. \& Velleman, A. Y. (1988). The Data Desk Handbook. Ithaca, N.Y.: Data Description, Inc.

Vincente, K. J. (1988). Adaptine the De Groot memory recall paradiem to evaluate interface transparency. (Report No. RISO-M-2691). Roskilde, Denmark: Riso National Laboratory

Wesson, R. B., \& Young, D. (1988). TRACON Air Traffic Control Simulation [Computer program]. Austin, TX: Wesson International.

Whitfield, D. (1979). A preliminary study of the air traffic controller's picture. CATCA Journal, 11, 19-28.

Whitfield, D., \& Jackson, A. (1982). The air traffic controller's 'picture' as an example of a mental model. In G. Johannsen \& J. E. Rijnsdorp (Eds.), Analysis, Design, and Evaluation of Man-Machine Systems (pp. 45-52). Duesseldorf, W. Germany: International Federation of Automatic Control.

Whitfield, D., \& Stammers, R. B. (1978). The air-traffic controller. In W. T. Singleton (Ed.), The Analysis of Practical Skills (pp. 209-235). Lancaster, UK: MTP.

Wilson, J. R., \& Rutherford, A. (1990). Mental Models: Theory and application in human factors. Human Factors, 31, 617-634.

Wickens, C. D. (1984). Engineering Psychology and Human Performance. Columbus, OH: Charles E. Merrill.

Wickens, C. D., \& Weingartner, A. (1985). Process control monitoring: the effects of spatial and verbal ability and concurrent task demand. Manuscript submitted for publication.

Witkin, H., A, Moore, C. A., Goodenough, D. R., \& Cox, P. W. (1977). Fielddependent and fiel-independent cognitive styles and their educatonal implications. Review of Educational Research, 47, 1-64.

Yuille, J. C. (1985). A laboratory-based experimental methodology is inappropriate for the study of mental imagery. Joumal of Mental Imagery, 2, 137-150.

Zubritzky, M. C., \& Coury, B. G., (1987). Multidimensional scaling as a method for probing the conceptual structure of state categories: An individual differences analysis. Proceedings of the Human Factors Society (pp. 107-111). Santa Monica, CA: Human Factors Society. 

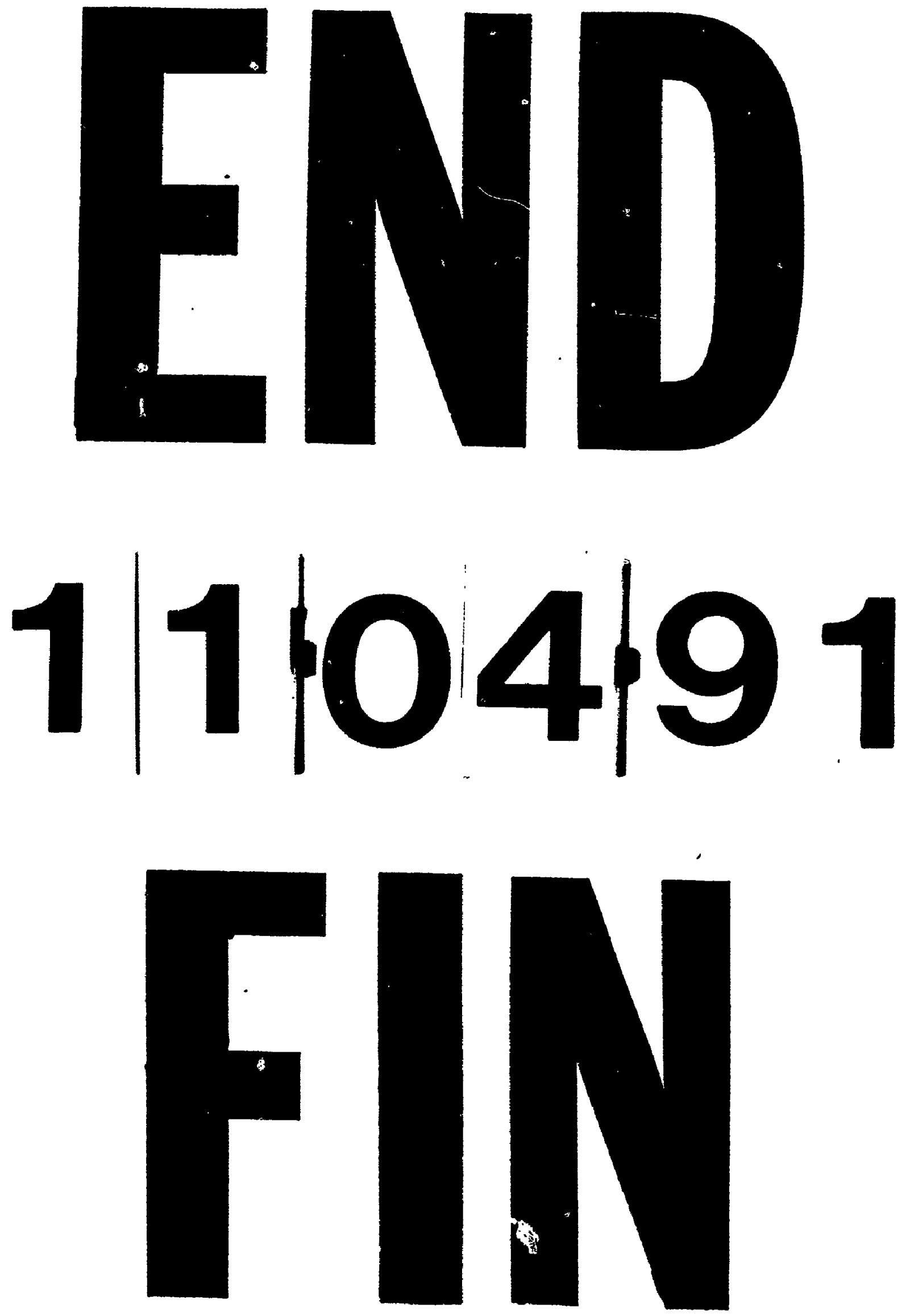